Universidade de São Paulo

Instituto de Astronomia, Geofísica e Ciências Atmosféricas

Departamento de Astronomia

Raniere Maciel de Menezes

\title{
Exploring the extreme Universe with the Fermi Large Area Telescope
}

São Paulo 

Raniere Maciel de Menezes

\section{Exploring the extreme Universe with the Fermi Large Area Telescope}

Corrected version. The original document is available at IAG-USP. (Versāo Corrigida. O original encontra-se disponível na Unidade)

Thesis presented to the Departament of Astronomy of the University of São Paulo in partial fulfillment of the requirements for the degree of Doctor of Philosophy

In the subject of: Astronomy \& Astrophysics Advisor: Professor Rodrigo Nemmen

São Paulo 

To the memory of Carl Sagan, whose efforts in spreading science were able to reach me 10 years after his death, in the depths of the sertão pernambucano. 



\section{Acknowledgements}

"Onde for, ande direito" said my old father when I left home on my way to Italy. I could not andar direito if it wasn't for you, mommy and Raí. Thank you. I would like also to thank my aunts, uncles and cousins for all the fondness and support during my whole life. A special thanks to Lica, whose hard work literally makes my life easier. I am also really grateful for what I have learned from my friends in Pesqueira. Whenever I remember our old times playing football, fishing and exploring those wild hills, the only feeling coming to my mind is the untranslatable Portuguese word saudade. Regarding the school, I would like to thank my teachers of Math and Geography, José Lopes and Maria da Paz. Even today I still remember important details from your lessons. A big thanks to my dear friends Carol, Clô, Landau, Lindão, Lucão, Mario, Natália, Rafa Bandeira, Rafa Rechiche and Vitão. You are among the smartest and kindest people I have ever met. For me, you are the real joy that I found across the event horizon of the university. Thank you very much to my office mates André, Henrique, Mirian, Pablo and Rogério. After so many years sitting by your side, I feel like you are a branch of my family in São Paulo. Thank you to my research teammates Artur, Fabito, Gus, Ivanildo, Malu Gubolin and Roberta. We have been sailing in the same spaceship for many years. Thank you for holding up the sails during the solar storms and for the many funny moments. A special thanks to Querido Jhon and Leo Santos, the best observational astronomers I have ever met. It would be a pleasure and an honor for me if we could work together someday. Grazie mille a tutti gli amici che ho trovato in Italia, soprattutto ad Alessandro, Ana, Ezequiel, Gian Luca, Giovanni, Harold, Leonardo, Marta, Romana, Valentina Cesare, Valentina Missaglia, Vittoria e Domenico Maisano. Peccato, perchè stavo bene in loro compagnia. My sincere thanks to Prof. Rama and the staff at the OAM. The project Noite com as Estrelas is by 
far the best science outreach project I have ever seen, and being part of this team was one of the most significant things for me during my stay in São Paulo. A great thanks to the staff at the Department of Astronomy, especially to Marco and Ulisses, who helped me several times with every kind of software and hardware issues. My most sincere thanks to the Fermi-LAT Collaboration, in particular to Liz Hays, Bindu Rani and Teddy Cheung. Thank you for the wonderful opportunities and especially for helping me to improve the level of my science. A kind thanks to Prof. Zulema, who has been accompanying my work and giving me important advice for the last four years. And finally, a very special thanks to those who taught me how to science, especially to Flávio Aguiar, Francesco Massaro, Raffaele D'Abrusco, and Rodrigo Nemmen.

This thesis was supported by CNPq (Conselho Nacional de Desenvolvimento Científico e Tecnológico) and FAPESP (Fundação de Amparo à Pesquisa do Estado de São Paulo) under grants 2016/25484-9 and 2018/24801-6. 
"Quando eu te encarei frente a frente, não vi o meu rosto. Chamei de mau gosto o que vi, de mau gosto, mau gosto. É que Narciso acha feio o que não é espelho."

Caetano Veloso

"Veloso, o Sol não é tão bonito pra quem vem do Norte e vai viver na rua."

Belchior 



\section{Abstract}

High-energy astrophysical processes commonly happen in the surroundings of compact objects, like neutron stars and black holes. In such environments, relativistic particles can emit $\gamma$-rays and also scatter low-energy photons up to the $\gamma$-ray domain. With the launch of the Fermi Large Area Telescope (LAT) in 2008, a new window was open to the $\gamma$-ray sky, allowing us to observe a huge variety of astrophysical phenomena never seen before. Fermi-LAT's remarkable sensitivity in the $100 \mathrm{MeV}-500 \mathrm{GeV}$ energy band allowed for the detection of more than $5000 \gamma$-ray sources and this number is still growing. In this thesis, we use the unprecedented Fermi-LAT capabilities in two different ways. In the first half of the thesis, we explore the $\gamma$-ray emission from globular clusters and low-luminosity active galactic nuclei (LLAGNs), where both classes are poorly understood in the high-energy domain. By using roughly 10 years of $\gamma$-ray observations, we are able to uncover some aspects of the dynamical formation of millisecond pulsars (MSPs) in globular clusters, especially on how stellar close encounters in the cores of globular clusters can form and/or disrupt compact mass-transferring binary systems, which could potentially evolve into $\gamma$-ray emitting MSPs. With a similar analysis, we explore the mechanisms behind the $\gamma$-ray emission of LLAGNs, this time also comparing hadronic and leptonic models for high-energy processes happening in the black hole surrounding hot accretion flow. In the second half of the thesis, we use Fermi-LAT data to associate $\gamma$-ray sources with their optical-infrared counterparts. Although the Fermi-LAT spatial resolution, typically $\lesssim 5^{\prime}$, is the highest ever achieved for a $\gamma$-ray space-based telescope, it is not enough to avoid problems with source association. In fact, $25 \%$ of the $\gamma$-ray sources listed in the last Fermi-LAT catalog have no obvious low-energy counterpart. To face this problem, we work on three distinct, direct and indirect, counterpart identification methods. At first, we use the correlations between 
mid-infrared colors and $\gamma$-ray emission to better select and characterize the counterpart candidates of Fermi-LAT blazars. We then develop an alternative method of counterpart association by accounting for the mid-infrared colors and radio loudness when computing the association probability with the likelihood ratio method. Finally, we perform a direct search for counterparts by doing an optical spectroscopic follow up of a few tens of sources. The results from these approaches are very promising, especially on the association and selection of future targets for spectroscopic follow up of Fermi-LAT blazars. 


\section{Resumo}

Processos físicos de altas energias comumente ocorrem nas redondezas de objetos astronômicos compactos, como estrelas de nêutrons e buracos negros. Nesses ambientes extremos, partículas subatômicas viajando a velocidades relativísticas são capazes de emitir raios $\gamma$, bem como ricochetear fótons de baixas energias, tornando-os muito mais energéticos. Com o lançamento do Fermi Large Area Telescope (LAT) em 2008, a observação de novos fenômenos astrofísicos tornou-se possível. A sensitividade sem precedentes do Fermi-LAT na banda de $100 \mathrm{MeV}$ - $500 \mathrm{GeV}$ permitiu a detecção de mais de 5000 fontes de raios $\gamma$ ao longo de mais de uma década de operações, número este que continua crescendo. Nesta tese de doutorado, nós usamos o potencial do Fermi-LAT de duas maneiras diferentes. $\mathrm{Na}$ primeira metade da tese, exploramos a emissão de raios $\gamma$ de aglomerados globulares e de núcleos ativos de galáxias com baixa luminosidade (LLAGNs), ambas as classes ainda pouco compreendidas no domínio dos raios $\gamma$. Usando aproximadamente 10 anos de observações, nós conseguimos desvendar alguns dos aspectos dinâmicos que levam à formação de pulsares de milissegundo (MSPs) em aglomerados globulares, e especialmente como encontros estelares nos núcleos desses aglomerados podem levar à formação e/ou desmembramento de sistemas binários compactos que, dependendo de sua configuração, podem evoluir para MSPs emissores de raios $\gamma$. Partindo de uma análise similar, nós exploramos os mecanismos de produção de raios $\gamma$ em LLAGNs, mas dessa vez testando modelos hadrônicos e leptônicos na tentativa de discriminar quais processos astrofísicos de altas energias geram os raios $\gamma$ que observamos nesses ambientes. Na segunda metade da tese, nós usamos os dados do Fermi-LAT para associar fontes de raios $\gamma$ às suas contrapartidas no infravermelho. Apesar dos excelentes avanços do Fermi-LAT em relação aos seus predecessores, sua resolução não é alta o suficiente para evitar o problema de 
associação de contrapartidas de baixas energias. De fato, cerca de $25 \%$ das fontes listadas no último catálogo do Fermi-LAT não possuem uma contrapartida óbvia em baixas energias. Para lidar com esse problema, nós trabalhamos com três métodos distintos de identificação de contrapartidas. Primeiro, usamos correlações entre cores no infravermelho e emissão de raios $\gamma$ para melhor caracterizar os candidatos a contrapartida de blazares do Fermi-LAT. Nós então desenvolvemos um método estatístico alternativo de associação de contrapartidas, onde levamos em conta cores no infravermelho e o comportamento no rádio das possíveis contrapartidas de fontes de raios $\gamma$. Por último, realizamos uma busca direta por candidatos a contrapartidas de blazares através de observações espectroscópicas de algumas dezenas de alvos. Os resultados de nossa busca se demonstraram muito promissores, principalmente na associação e seleção de alvos para futuras missões de varredura espectroscópica do céu de altas energias. 


\section{Contents}

1. The gamma-ray sky in the Fermi-LAT era . . . . . . . . . . . . . . . . . . . 19

1.1 History of gamma-ray observations . . . . . . . . . . . . . . 20

1.2 Current gamma-ray observatories . . . . . . . . . . . . . . . . . 22

1.2.1 Fermi-LAT . . . . . . . . . . . . . . . . . . 23

1.2.1.1 Tracker . . . . . . . . . . . . . . . . 24

1.2.1.2 Calorimeter . . . . . . . . . . . . . . 25

1.2.1.3 Anticoincidence detector (ACD) . . . . . . . . 26

1.2.2 Ground-based observatories . . . . . . . . . . . . . . . . . . 27

2. Relevant sources and key science problems . . . . . . . . . . . . . . . . . . 31

2.1 Globular clusters . . . . . . . . . . . . . . . . . . . 32

2.1.1 Pulsars: back-of-the-envelope calculations . . . . . . . . . . . 33

2.1.2 Magnetospheric gamma-rays . . . . . . . . . . . . . . . . . . 38

2.1.3 Dynamical formation and evolution of MSPs . . . . . . . . . . . . 39

2.2 Low-luminosity active galactic nuclei (LLAGNs) . . . . . . . . . . . . . . 41

2.2.1 Radiatively inefficient accretion flow (RIAF) . . . . . . . . . . . . 42

2.3 Unassociated gamma-ray sources (UGSs) . . . . . . . . . . . . . . 45

3. Physics of gamma-ray emission . . . . . . . . . . . . . . . . . . . 49

3.1 Outer gap acceleration and curvature radiation . . . . . . . . . . . . 49

3.2 Pion decay in hot accretion flows . . . . . . . . . . . . . . . 51

3.3 Inverse Compton scattering . . . . . . . . . . . . . . . 53

3.3.1 Synchrotron Self-Compton and external Compton . . . . . . . . . 56

$3.4 \gamma-\gamma$ absorption . . . . . . . . . . . . . . . . . . 56 
4. The gamma-ray emission from globular clusters . . . . . . . . . . . . . . . . 59

4.1 Introduction . . . . . . . . . . . . . . . . . . 60

4.2 Data selection and analysis . . . . . . . . . . . . . . 61

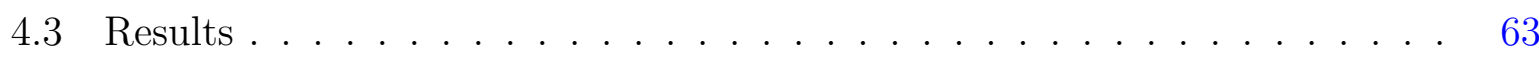

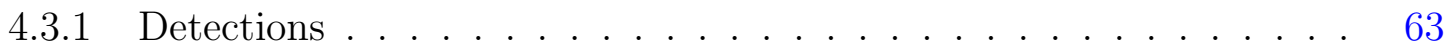

4.3.1.1 Fermi-LAT 8 years source list . . . . . . . . . 64

4.3.2 Point-like sources . . . . . . . . . . . . . . . . 64

4.3.3 Extended source analysis . . . . . . . . . . . . . 65

4.3.4 Light curves . . . . . . . . . . . . . . . . . . . . . . 65

4.3.5 Spectral emission models . . . . . . . . . . . . . . 66

4.3.6 Correlated quantities . . . . . . . . . . . . . . 67

4.3.7 Secondary encounters and binary disruption . . . . . . . . . . . 70

4.4 Discussion . . . . . . . . . . . . . . . . . . . . 70

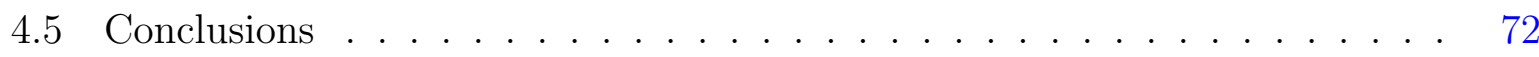

4.6 Appendix: Upper limits on non-detected GCs and SEDs . . . . . . . . . 73

5. The gamma-ray emission from LLAGNs . . . . . . . . . . . . . . . . . . 79

5.1 Introduction . . . . . . . . . . . . . . . . . . . 80

5.2 Fermi-LAT observations . . . . . . . . . . . . . . . . 83

5.3 X-ray and radio data . . . . . . . . . . . . . . . . . . . 84

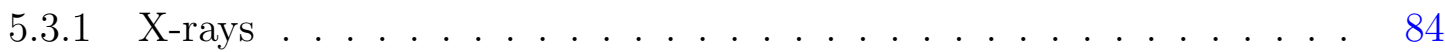

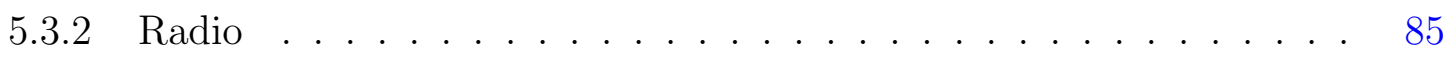

5.4 Spectral models . . . . . . . . . . . . . . . . . 85

5.5 Results . . . . . . . . . . . . . . . . . . . . 88

5.6 Discussion . . . . . . . . . . . . . . . . . . . . . . . 94

5.7 Conclusions . . . . . . . . . . . . . . . . . . . . 96

5.8 Appendix: Energy flux upper limits for all AGN non-detected in gamma-rays 98

6. The infrared colors of gamma-ray blazars . . . . . . . . . . . . . . . . . . . . . . 99

6.1 Introduction . . . . . . . . . . . . . . . . . . 100

6.2 Sample selection . . . . . . . . . . . . . . . . . . . 102

6.2 .1 WIBRaLS2 . . . . . . . . . . . . . . 102

6.2 .2 KDEBLLACS . . . . . . . . . . . . . . . . 103 
6.3 Spectral analysis and classification . . . . . . . . . . . . . 105

6.4 Results . . . . . . . . . . . . . . . . . . 107

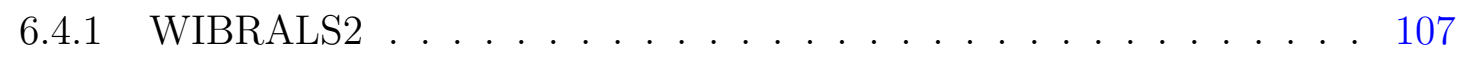

6.4 .2 KDEBLLACS . . . . . . . . . . . . . . . . . . 108

6.4.3 Selection effects . . . . . . . . . . . . . . . . . . 109

6.4.4 Blazar candidates of uncertain type . . . . . . . . . . 111

6.4 .5 New BZBs . . . . . . . . . . . . . . . . 112

6.5 Summary and conclusions . . . . . . . . . . . . . . 113

7. On the association of gamma-ray sources . . . . . . . . . . . . . . 117

7.1 Introduction . . . . . . . . . . . . . . . . 118

7.2 Samples used for the likelihood ratio method . . . . . . . . . . . . 120

7.3 Samples used for the supervised learning method . . . . . . . . . . . . 121

7.4 The likelihood ratio method . . . . . . . . . . . . . . . . 122

7.5 The random forest method . . . . . . . . . . . . . . 126

7.6 Results from the likelihood ratio approach . . . . . . . . . . . . 127

7.6.1 Considering angular separation . . . . . . . . . . . . 127

7.7 Results from the random forest approach . . . . . . . . . . . . . 128

7.8 Discussion and conclusions . . . . . . . . . . . . . . . 130

7.9 Appendix: List of targets for optical spectroscopic follow up . . . . . . . 132

8. Spectroscopic follow-up of BCUs . . . . . . . . . . . . . . . . . 133

8.1 Introduction . . . . . . . . . . . . . . . . 134

8.2 Sample selection . . . . . . . . . . . . . . . 136

8.3 Observations and data reduction . . . . . . . . . . . . . 138

8.4 Analysis and results . . . . . . . . . . . . . . . . . 140

8.5 Summary and Conclusions . . . . . . . . . . . . . . . 143

9. Conclusion and future directions . . . . . . . . . . . . . . . . . . 145

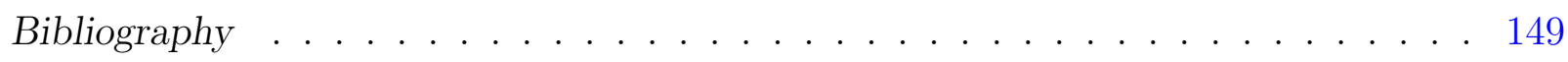





\section{List of abbreviations}

3FGL: Fermi-LAT 3rd Source Catalog

4FGL: Fermi-LAT 4th Source Catalog

ACD: Anticoincidence detector

ADAF: Advection-dominated accretion flow

AGN: Active galactic nucleus

AP: Association probability

BCU: Blazar candidate of uncertain type

CGRO: Compton Gamma-Ray Observatory

CTA: Cherenkov Telescope Array

EGRET: Energetic Gamma Ray Experiment Telescope

FSRQ: Flat-spectrum radio quasar

IACT: Imaging Atmospheric Cherenkov Telescopes

KDEBLLACS: Kernel density estimation selected candidate BL Lacs

LAT: Large Area Telescope

LLAGN: Low-luminosity active galactic nucleus

LMXB: Low-mass X-ray binary

MIR: Mid-infrared

MSP: Millisecond pulsar

PSF: Point-spread function

RIAF: Radiatively inefficient accretion flow

SDSS: Sloan Digital Sky Survey

SED: Spectral energy distribution

SMBH: Supermassive black hole 
SSC: Synchrotron self-Compton

UGS: Unidentified $\gamma$-ray source

VHE: Very high energy

WIBRaLS: WISE Blazar-like Radio-Loud Sources

WISE: Wide-field Infrared Survey Explorer 
Chapter 1

\section{The gamma-ray sky in the Fermi-LAT era}

The Universe shines in $\gamma$-rays. We have been, however, blind to such energies until the late 1950's, when balloon and space-based observations revealed the first astronomical $\gamma$-rays (Peterson and Winckler, 1959; Kraushaar and Clark, 1962). In fact, $\gamma$-rays were the last electromagnetic window to be explored by astronomers, appearing on stage chronologically after X-rays (Chubb et al., 1957), ultraviolet radiation (UV; Allen, 1946), radio waves (Jansky, 1932), infrared radiation (IR; Nichols, 1901, and references therein) and, of course, optical (since we first glanced at the sky).

As the most energetic form of electromagnetic radiation, $\gamma$-rays are fundamental in the understanding of non-thermal astrophysical processes, like relativistic jets launched by active galactic nuclei (AGNs), supernova explosions and remnants, magnetospheric acceleration of particles in the surroundings of pulsars, and even exotic phenomena like dark matter annihilation. Unaffected by magnetic fields and exploiting the transparency of the local Universe at this energy band, $\gamma$-rays bring information directly from their sources, with no deflection or absorption in their way.

Given that the Earth's atmosphere is opaque to photons at these energies, the direct detection of $\gamma$-rays must be carried out from space, while indirect detection can be performed by ground-based Cherenkov telescopes. Launched in 2008, the Fermi Gamma-ray Space Telescope (Fermi, for short) has surveyed the entire $\gamma$-ray sky on a daily basis, vastly improving our knowledge on high energy astrophysics. The primary instrument on board Fermi is the Large Area Telescope (Fermi-LAT; Atwood et al., 2009), which by now has detected more than 5000 permanent sources and provided spectral and temporal information on all of them. The objects detected by Fermi-LAT form a zoo of astronomical sources which include the Sun, the Moon, pulsars, supernova remnants, high-mass binaries, 
globular clusters, novae and nebulae within the Milky Way, while the extragalactic $\gamma$-ray sky is dominated by AGNs, $\gamma$-ray bursts, and presents a few starburst and normal galaxies (Ackermann et al., 2012; Abdollahi et al., 2020). All these sources are seen against two diffuse $\gamma$-ray backgrounds: the Galactic background, originated from cosmic-ray interactions with the interstellar medium, and the isotropic extragalactic background whose origin is still not fully understood. The secondary instrument on board Fermi is the Gamma-ray Burst Monitor, which extends the energy range of Fermi-LAT down to the hard X-ray band and focus specifically on the characterization and localization of $\gamma$-ray bursts.

We start this thesis by introducing the history of $\gamma$-ray observations and how modern $\gamma$ ray observatories operate in $\S 1.1$ and $\S 1.2$. We then review the relevant source populations and discuss the key science questions for our work in Chapter 2. The physics of $\gamma$-ray emission is explored in Chapter 3. In Chapters 4, 5, 6, 7 and 8 we detail the main papers published as a result of our research. In Chapter 9 we discuss and summarize our work.

\subsection{History of gamma-ray observations}

Long before the first astrophysical observations of $\gamma$-rays (see Peterson and Winckler, 1959, for early balloon observations), scientists knew a number of astrophysical processes which could produce photons with energies above $\sim 1 \mathrm{MeV}$, like supernova explosions and cosmic ray interactions with the interstellar medium, to cite a few. In the early 1960's, we developed the ability to detect $\gamma$-rays from space with the launch of the Explorer 11 satellite (Kraushaar and Clark, 1962; Kraushaar et al., 1965), which detected around 100 $\gamma$-rays from outside the Solar System and arriving in random directions, implying a sort of uniform $\gamma$-ray background.

Significant $\gamma$-ray emission from the Galactic plane was first published by Clark et al. (1968), using data collected with the Orbiting Solar Observatory 3 (OSO-3) satellite, at energies above $\sim 70 \mathrm{MeV}$. The first extragalactic $\gamma$-rays were also detected by $O S O-3$ (Kraushaar et al., 1972), and appeared as an isotropic component with a softer energy spectrum than that of the Galaxy. Later in the 1970's, observations with the Small Astronomy Satellite 2 (SAS-2) and the Celestial Observation Satellite (COS-B) confirmed these results (Thompson and Fichtel, 1982) and found a few point sources at a spatial resolution $>1^{\circ}$, including the first extragalactic $\gamma$-ray source, the blazar 3C 273 (Swanenburg et al., 

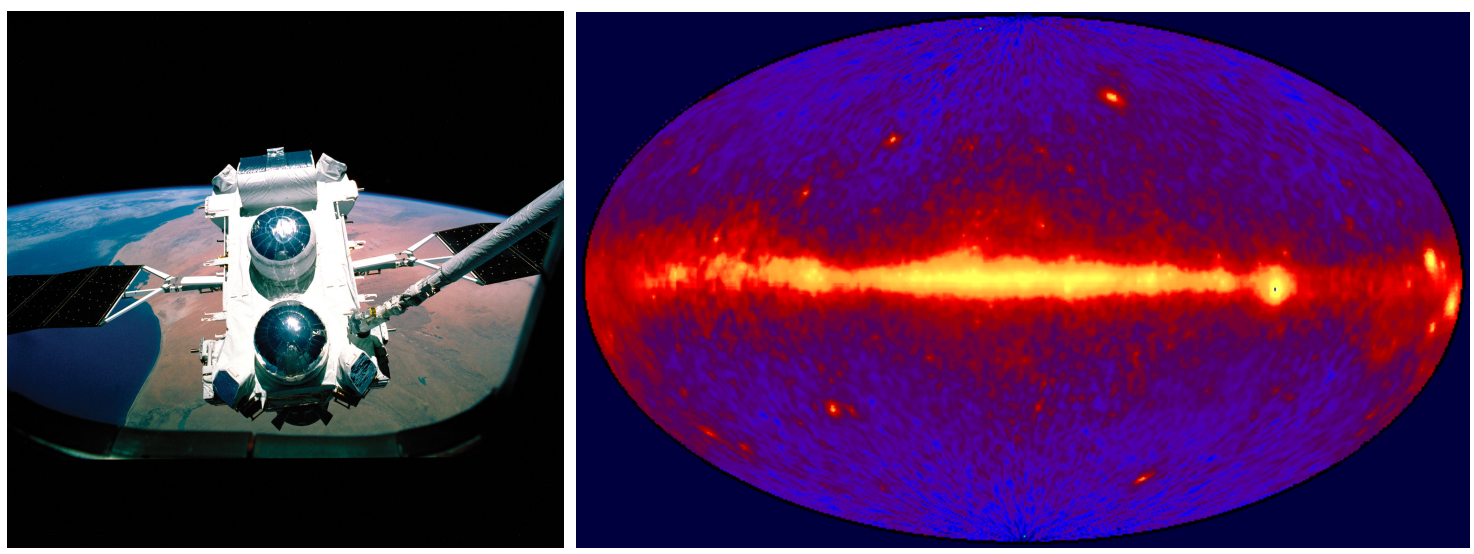

Figure 1.1: The CGRO mission. Left: deployment of the CGRO from the space shuttle Atlantis on April, 1991. Right: all-sky map in Galactic coordinates obtained by EGRET on board CGRO at energies above $100 \mathrm{MeV}$. The plane of the Milky Way is clearly seen as a strong source of diffuse and resolved emission. Credit: Compton Gamma Ray Observatory Team, in public domain.

1978), and raising the possibility that the isotropic extragalactic background consisted of unresolved active galaxies.

In 1977, NASA announced the plans for building the Compton Gamma-Ray Observatory (CGRO; see Figure 1.1) as part of the High Energy Astronomy Observatory Program (HEAO; Neighbors et al., 1977). The observatory was designed to take advantage on the improvements on detector technology during the 1980s. CGRO was launched in 1991 and carried four instruments that covered an unprecedented six decades of the electromagnetic spectrum, from $20 \mathrm{keV}$ to $30 \mathrm{GeV}$, and greatly improved the spatial and temporal resolution of $\gamma$-ray observations, showing the high-energy $\gamma$-ray sky as a surprisingly dynamic and diverse environment. The first all-sky survey in $\gamma$-rays was performed by the Energetic Gamma Ray Experiment Telescope (EGRET), on board CGRO, and can be checked in Figure 1.1. The $\gamma$-ray sources observed with EGRET are summarized in the Third EGRET Catalog (3EG; Hartman et al., 1999). In June 2000, CGRO was deorbited due to problems with one of its gyroscopes.

The next generation of space-based $\gamma$-ray observatories (above a few MeV) came with the launch of Fermi in 2008. While EGRET detected less than $300 \gamma$-ray sources (see Hartman et al., 1999, and Casandjian and Grenier, 2008, for different analyses with significantly different results), Fermi-LAT has discovered more than 5000 sources to date (Abdollahi et al., 2020) and the number is still increasing. Fermi-LAT presents a broader energy range and field of view than EGRET, a much larger effective area, a smaller dead time per trigger, and significantly better angular resolution (Atwood et al., 2009). While it took EGRET 
one year and a half to release its first $\gamma$-ray all-sky map, Fermi-LAT maps the whole sky about every three hours since the beginning of the mission. Parallel to Fermi-LAT, the Astro-rivelatore Gamma a Immagini LEggero (AGILE; Tavani et al., 2008), launched in 2007, has also contributed to a number of studies in $\gamma$-ray astronomy (see Tavani, 2019, for a review), although working with a smaller energy range and sensitivity than Fermi-LAT. Technical details on Fermi-LAT can be found in $\S 1.2$.

Simultaneously to the advances in space-based $\gamma$-ray astronomy, ground-based $\gamma$-ray observations with Imaging Atmospheric Cherenkov Telescopes (IACTs) started to give their first results in the late 1980's (Weekes et al., 1989). Since then, very high energy (VHE) astronomy came into reality and the number of VHE sources has grown up to $\sim 200^{1}$. These telescopes do not directly detect the GeV-TeV $\gamma$-rays, but the Cherenkov light emitted by cascades of particles resulting from the interaction of a primary $\gamma$-ray with atomic nuclei in the Earth's atmosphere (see more details in $§ 1.2$ ). The main IACTs in operation to date are the Major Atmospheric Gamma Imaging Cherenkov Telescopes (MAGIC; Baixeras et al., 2003), the High Energy Stereoscopic System (H.E.S.S.; Hinton et al., 2004), and the Very Energetic Radiation Imaging Telescope Array System (VERITAS; Holder et al., 2006). In the forthcoming years, with more than 100 telescopes located in both hemispheres, the Cherenkov Telescope Array (CTA; Actis et al., 2011) will be the world's largest and most sensitive VHE $\gamma$-ray observatory.

\subsection{Current gamma-ray observatories}

Modern $\gamma$-ray observations are carried on from ground and space-based observatories. Together, Fermi-LAT and IACTs cover an energy range from $\sim 20 \mathrm{MeV}$ up to $\lesssim 100$ $\mathrm{TeV}$. This range can be extended in both directions if we consider $\gamma$-ray burst detectors (e.g., Meegan et al., 2009) and water-based Cherenkov observatories (e.g., DeYoung et al., 2012), but these types of observatories are not closely related with the main topics of this thesis. In the following, we describe how Fermi-LAT and IACTs work.

\footnotetext{
${ }^{1}$ http://tevcat.uchicago.edu/
} 

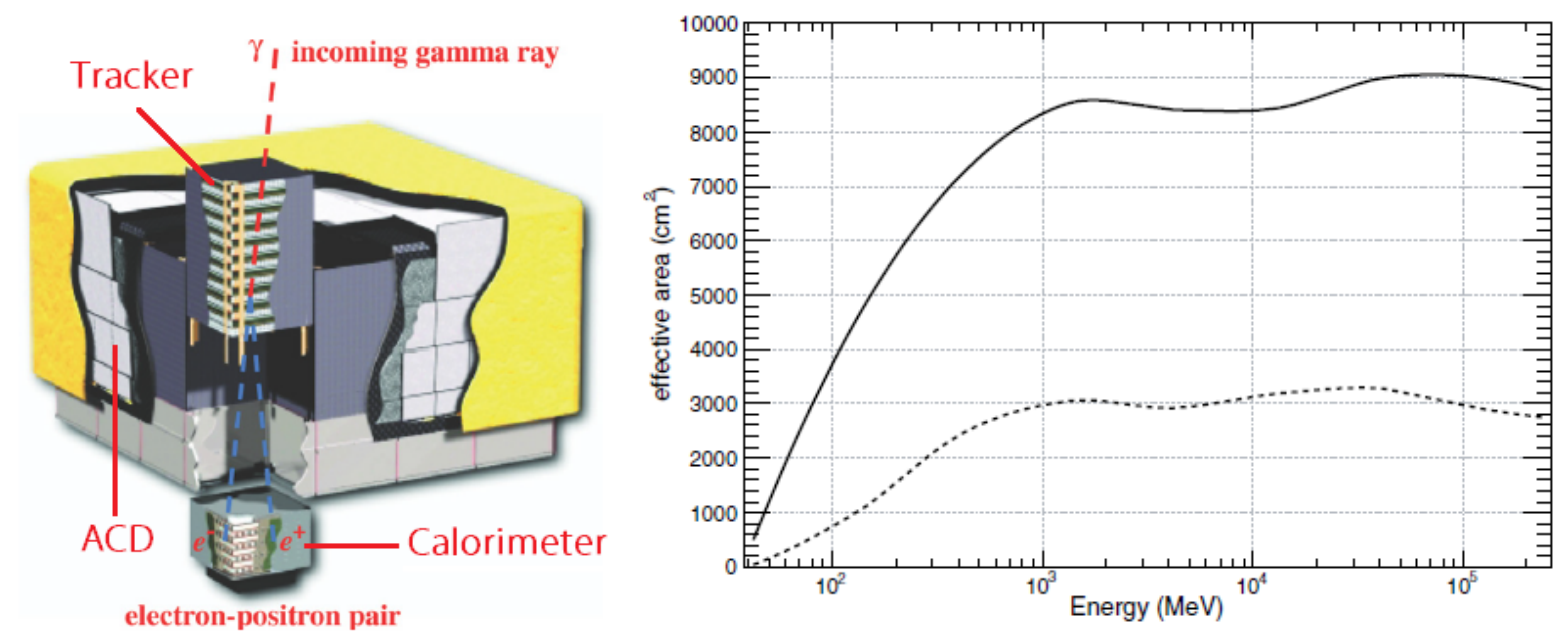

Figure 1.2: The Fermi-LAT. Left: cartoon with all Fermi-LAT parts assembled together and covered by the micrometeoroid shield (yellow). Right: effective area vs. energy at normal incidence (solid curve) and at $60^{\circ}$ off-axis (dashed curve) for Source analysis class. Images modified from Atwood et al. (2009).

\subsubsection{Fermi-LAT}

The Fermi-LAT consists of three main parts: i) a $4 \times 4$ array of identical tracker towers, where $\gamma$-rays interact via pair production $\left(\gamma \rightarrow e^{+}+e^{-}\right)$and the resulting particles are tracked down to point to the original arrival direction of the $\gamma$-ray photon (see §1.2.1.1); ii) a cesium iodide calorimeter where the energy of the particles and then the pair-converted photon is measured (see $\S 1.2 .1 .2$ ), and iii) the anticoincidence detector (ACD; see $§ 1.2 .1 .3$ ), which is a plastic scintillator covering the tracker towers, aiming to reject the huge cosmic ray background found in the low-Earth orbit of the satellite (see Atwood et al., 2009, for a very detailed review on the Fermi-LAT). In the left panel of Figure 1.2 we show all three parts assembled. The yellow structure covering the tracker is a micrometeoroid shield.

The Fermi-LAT achieves its maximum performance in the 1-10 GeV energy range, where the photon count is relatively high, the effective area is $\sim 9000 \mathrm{~cm}^{2}$ (see right panel of Figure 1.2), the field of view is $2.4 \mathrm{sr}$ (or $19 \%$ of the whole sky), the energy resolution is better than 10\%, and the single-photon angular resolution (68\% containment angle) is $0.6^{\circ}$ (see Atwood et al., 2009, for more details). The Fermi-LAT specifications are shown in Table 1.1, where we also do a comparison with its predecessor EGRET.

In general, $\gamma$-rays cannot be refracted or reflected because they pair-produce once they interact with matter. It is impossible to converge them into a focal point and hence get amplified images. The only mechanism able to converge $\gamma$-rays into a focal point is gravitational lensing, which converges light beams regardless of their photon frequencies, 


\begin{tabular}{l|l|l} 
Quantity & Fermi-LAT & EGRET \\
\hline Energy range & $20 \mathrm{MeV}-500 \mathrm{GeV}$ & $20 \mathrm{MeV}-30 \mathrm{GeV}$ \\
Peak effective area & $\sim 9000 \mathrm{~cm}^{2}$ & $\sim 1500 \mathrm{~cm}^{2}$ \\
Field of view & $2.4 \mathrm{sr}$ & $0.5 \mathrm{sr}$ \\
Angular resolution & & \\
- & $<3.5^{\circ}(100 \mathrm{MeV})$ & $5.8^{\circ}(100 \mathrm{MeV})$ \\
- & $0.6^{\circ}(1 \mathrm{Gev})$ & - \\
Energy resolution $^{3}$ & $\leq 0.15^{\circ}(>10 \mathrm{GeV})$ & - \\
Deadtime per event $^{2}$ & $818 \%$ & $18 \sim 25 \%$ \\
Source location determination $^{4}$ & $<26 \mu \mathrm{s} / \mathrm{event}$ & $100 \mathrm{~ms} / \mathrm{event}$ \\
Point source sensitivity $^{5}$ & $<3 \times 10^{-9} \mathrm{~cm}^{-2} \mathrm{~s}^{-1}$ & $\sim 15^{\prime}$ \\
& &
\end{tabular}

Table 1.1 - Specifications and performance of Fermi-LAT in comparison with EGRET. The superscripts mean 1) After background rejection, 2) Single photon, 68\% containment, on-axis, 3) $1 \sigma$, on-axis, 4) $1 \sigma$ radius, flux $10^{-7} \mathrm{~cm}^{-2} \mathrm{~s}^{-1}(>100 \mathrm{MeV})$, at high $\left.|b|, 5\right)$ for a steady source after one-year sky survey above $100 \mathrm{MeV}$, at high $|b|$ and photon index -2.1 with no spectral cutoff. Data collected from Atwood et al. (2009) and Thompson et al. (1993).

hence being a completely achromatic phenomenon (see Landis, 2017, for a critical analysis of using Sun's gravity as a giant telescope in the far future). The best one can do right now in $\gamma$-ray astronomy is to pinpoint the arrival direction photons with a spatial resolution of a few arcminutes. In the case of Fermi-LAT, this task is performed by the tracker.

\subsubsection{Tracker}

The arrival direction of a $\gamma$-ray is measured by the tracker. Each tracker tower has 16 pair-conversion planes of tungsten interleaved with 18 tracking planes of silicon strip detectors, as shown in the left panel of Figure 1.3. The high atomic number of tungsten increases the probability of pair production, as this probability is roughly proportional to the square of the atomic number. After the pair production in one of the tungsten layers, the produced $\mathrm{e}^{-}+\mathrm{e}^{+}$pair leaves ionized paths in the silicon strip layers which can be mapped down to reconstruct the arrival direction of the $\gamma$-ray.

Multiple scattering of the $\mathrm{e}^{-}+\mathrm{e}^{+}$pair and bremsstrahlung production limit the final resolution. The best results require the $\mathrm{e}^{-}+\mathrm{e}^{+}$directions to be measured immediately after the conversion, thus the silicon layers must be placed just below the tungsten layers. The point-spread function (PSF) is defined as the probability distribution for the reconstructed direction of an incident $\gamma$-ray from a point source, and the signature of the pair conversion is further used to help in rejecting the large background of cosmic rays. The higher the photon energy, the lower is the impact of multiple scatterings in the measurements of $\mathrm{e}^{-}+$ 

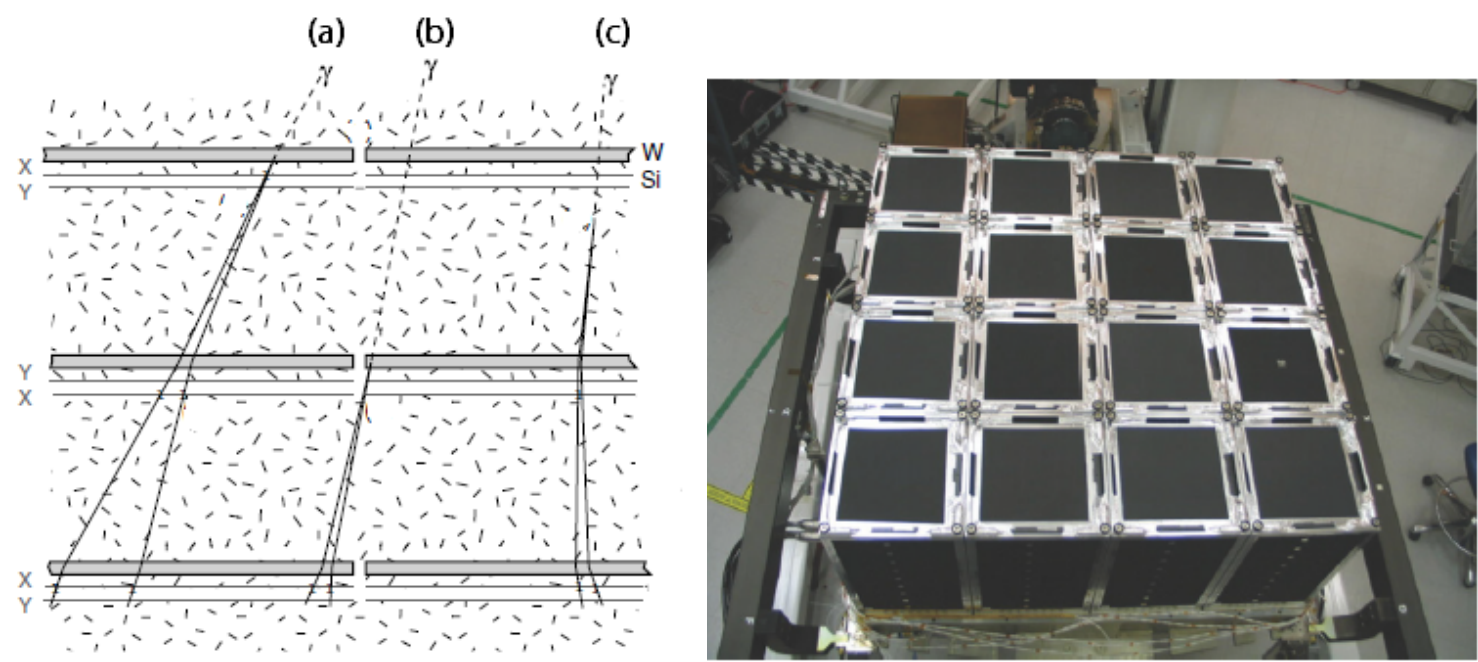

Figure 1.3: The tracker. Left: schematic of a tracker tower, where "W" represents the tungsten layers and "Si"the silicon tracking planes. The three cases shown are: (a) ideal conversion in $\mathrm{W}$, with the $\mathrm{Si}$ detector located as close as possible to the $\mathrm{W}$ foils, minimizing the lever arm for multiple scattering; (b) a missed hit in the first layer degrades the PSF but gives a reasonable result anyway; and (c) a conversion in the structural material or Si layers can give long lever arms for multiple scattering and degrade the PSF, so such material is minimized. Right: the $4 \times 4$ array of tracker towers before integration with the ACD. Images modified from Atwood et al. (2009).

$\mathrm{e}^{+}$directions, thus implying in a better PSF for higher energies (the PSF goes roughly with $1 / E$, where $E$ is the photon energy).

In the right panel of Figure 1.3 we show the $4 \times 4$ array of tracker towers before integration with the ACD. The whole structure is placed above the calorimeter, which has the task of reconstructing the energy of the incoming photon based on the particle cascade originated when the $\gamma$-ray pair-produces in one of the tungsten layers of the tracker. Each tracker tower has its own calorimeter module.

\subsubsection{Calorimeter}

The main goals of the calorimeter are (i) to measure the energy deposition by the electromagnetic shower resulted from the $\mathrm{e}^{-}+\mathrm{e}^{+}$pair, which is produced by the incident $\gamma-$ ray, and (ii) to roughly image the shower development profile, hence providing an important background discriminator. Each calorimeter module has $96 \mathrm{CsI}(\mathrm{Tl})$ scintillator crystals (cesium iodide activated with thallium) optically isolated from each other, and arranged in eight alternating orthogonal layers (see Figure 1.4).

At normal incidence the calorimeter's depth is 8.6 radiation lengths (increasing to 10.1 radiation lengths if we consider the depth of the whole Fermi-LAT). The shower imaging capability and depth of the calorimeter enable the high-energy reach (up to a couple TeV) 


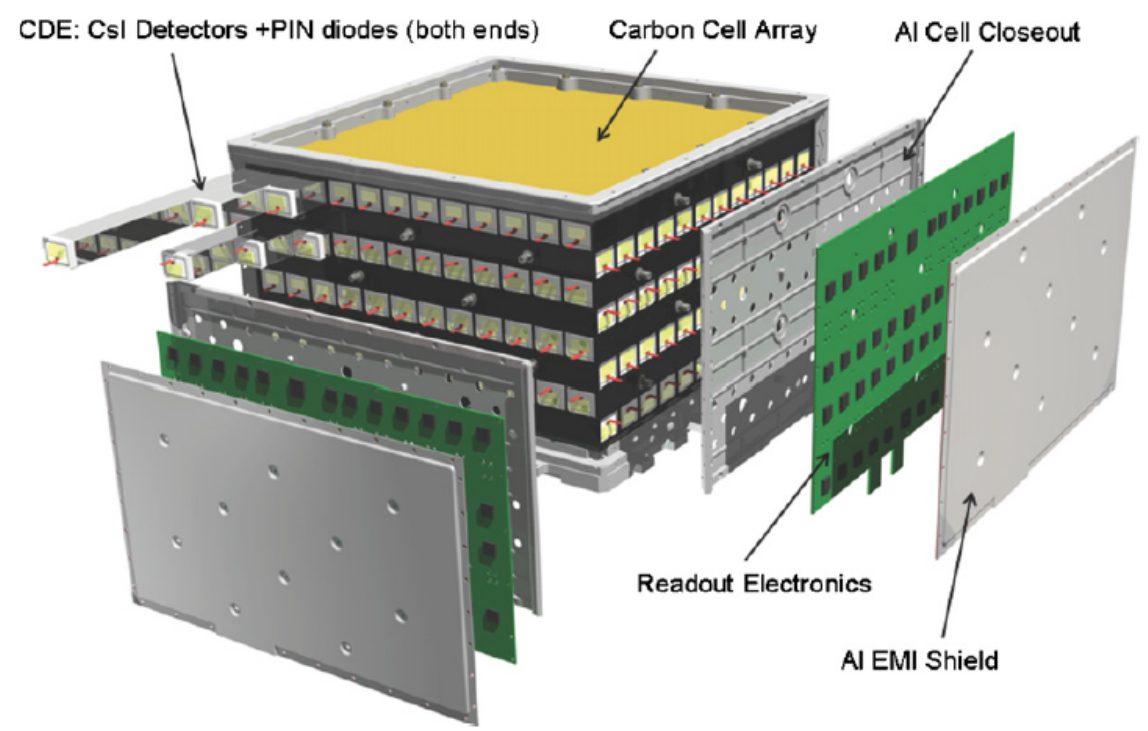

Figure 1.4: A calorimeter module. The $96 \mathrm{CsI}(\mathrm{Tl})$ scintillator crystals are arranged in eight alternating orthogonal layers. Each calorimeter module has a depth of 8.6 radiation lengths at normal incidence. Image from Atwood et al. (2009).

of the Fermi-LAT and contribute significantly to background rejection. By measuring the longitudinal shower profile and fitting the measurements to an analytical description of the cascade, an unbiased estimate of the initial $\mathrm{e}^{-}+\mathrm{e}^{+}$pair energy is obtained. Although the tracker and calorimeter can help in rejecting the particle background, the major defense of Fermi-LAT against cosmic rays is the ACD.

\subsubsection{Anticoincidence detector (ACD)}

The ACD is Fermi-LAT's best defense against the enormous cosmic ray background and presents an average efficiency of $99.97 \%$ for detection of charged particles entering the telescope's field of view. The ACD covers the 16 tracker towers with segmented tiles of plastic scintillator which produce a flash of light once they are traversed by a charged particle, but are transparent to the relatively rare $\gamma$-rays. When combined with the other subsystems, the Fermi-LAT total cosmic ray rejection becomes $99.999 \%$.

To minimize the probability of physical damage by micrometeorites and space debris, the ACD is covered by a low-mass shield, built in a way to avoid $\gamma$-ray production from cosmic ray interactions with the shield material. The characteristic $\gamma$-ray signature in the Fermi-LAT is then (i) no signal in the ACD, (ii) an electromagnetic profile for the cascade induced in the tracker, and (iii) an electromagnetic profile for the particle cascade in the calorimeter. 


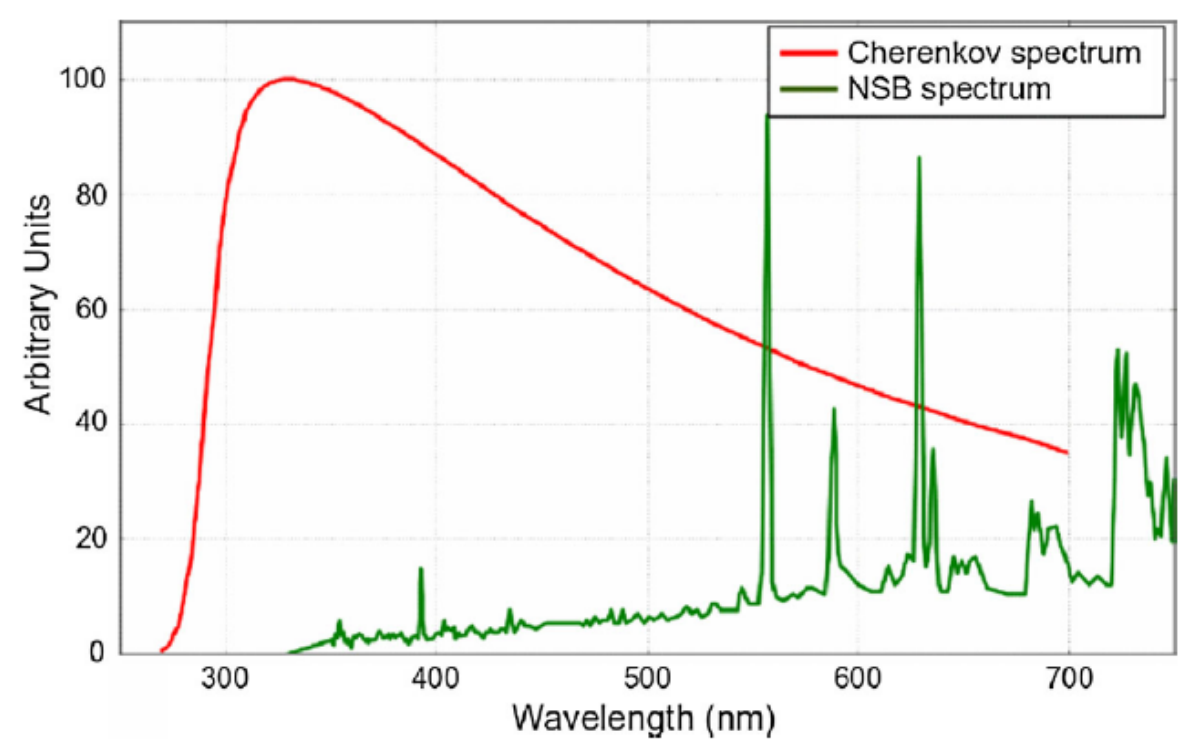

Figure 1.5: Cherenkov and night sky background (NSB) spectra in La Palma, Spain, at $2200 \mathrm{~m}$ above sea level. Image from Bonardi et al. (2014).

\subsubsection{Ground-based observatories}

Earth's atmosphere is opaque to $\gamma$-rays. At energies $\gtrsim 1 \mathrm{MeV}$ (i.e., more than twice the electron rest mass) the dominant photon-matter interaction is pair production. After a $\gamma$-ray pair-produces in the atmosphere, secondary particles give origin to a new generation of $\gamma$-rays via bremsstrahlung, which in turn can generate tertiary particles and so on. This process starts an electromagnetic shower with an increasing number of $\mathrm{e}^{-}+\mathrm{e}^{+}$ pairs and lower-energy $\gamma$-rays as a function of the atmospheric depth. The average energy of the secondary particles eventually drops to the point where the cross section for ionization losses exceeds those for bremsstrahlung, thus braking the further development of the shower. In this scenario, Earth's atmosphere behaves a bit like a tracker and a bit as a deep calorimeter with a few tens of radiation length (see $§ 1.2 .1 .1$ and $§ 1.2 .1 .2$ above).

In practice, IACTs can detect air showers produced by primary $\gamma$-rays with energies above a couple tens of $\mathrm{GeV}$, where we have a huge number of secondary particles traveling faster than light in the atmosphere. These faster-than-light particles create a blue flash of Cherenkov light (see the spectrum in Figure 1.5) typically spreading over an area with a few hundreds of meters in diameter and lasting for a few billionths of a second.

The main background for IACTs are air showers induced by cosmic rays. When a hadronic cosmic ray interacts with a nucleus in the Earth's atmosphere, it produces nuclear fragments and a number of pions with relatively large transverse momentum (see Aharonian 

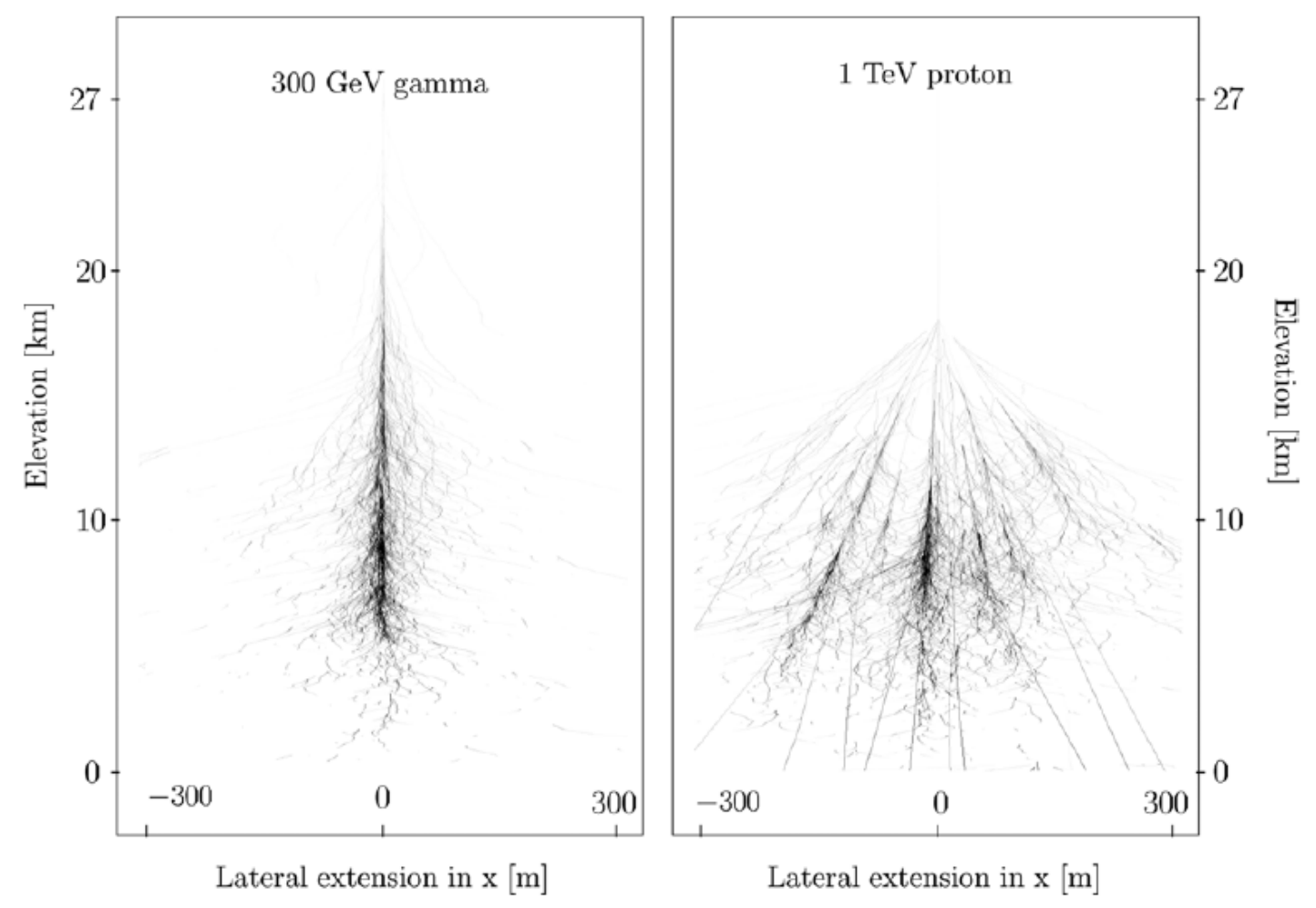

Figure 1.6: Electromagnetic (left) and hadronic (right) shower profiles. The shown electromagnetic shower simulates the interaction of a $300 \mathrm{GeV} \gamma$-ray with the Earth's atmosphere, while the hadronic shower simulates a similar scenario for a $1 \mathrm{TeV}$ proton. Image from Aharonian et al. (2008).

et al., 2008, for details). The neutral pions $\left(\pi^{0}\right)$ quickly decay into a pair of $\gamma$-rays, which in turn initiate electromagnetic cascades. Charged pions, on the other hand, decay typically to muons and neutrinos (i.e., $\pi^{+} \rightarrow \mu^{+}+\nu_{\mu}$ and $\pi^{-} \rightarrow \mu^{-}+\bar{\nu}_{\mu}$ ), and these muons give rise to compact circular patches of Cherenkov light at the ground level. Cosmic ray showers then project a more irregular structure of Cherenkov radiation at the ground if compared with electromagnetic showers (see Figures 1.6 and 1.7). The differences in the shape of both types of cascades is then used to distinguish between electromagnetic and hadronic showers.

By recording a snapshot of the Cherenkov light along the longitudinal development of the shower, it is possible to determine the energy and arrival direction of the primary $\gamma$ ray. Intrinsic fluctuations in the shower development as well as limited sampling with the ground-based detectors limit the energy resolution that can be achieved with the current generation of IACTs to the level of $\Delta E / E \sim 15 \%$ (Tridon et al., 2010). We expect to improve it to $\Delta E / E \sim 5 \%$ with the advent of CTA (Hassan et al., 2015).

Although we do not use IACT observations in this thesis, this is an important topic to be discussed, as we want to put our work in the perspectives of the CTA, which will 


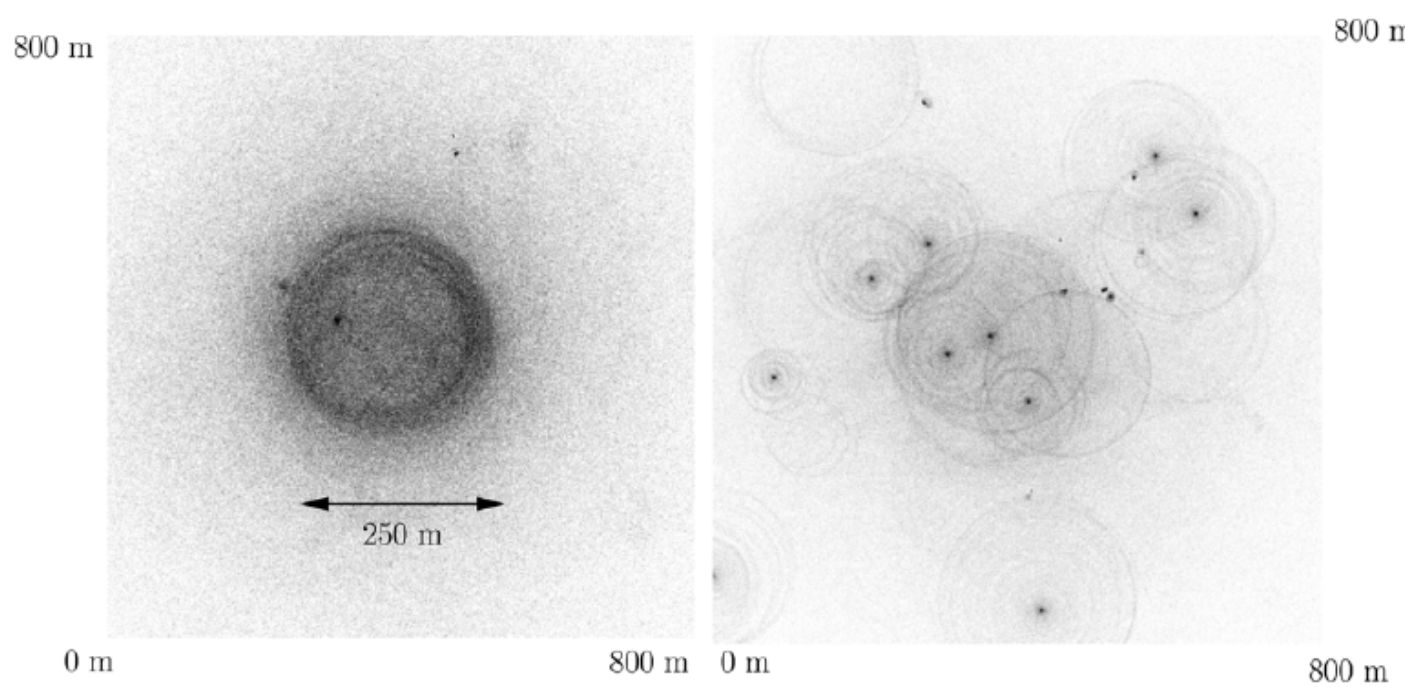

Figure 1.7: Ground-level distribution of Cherenkov light for the showers discussed in Figure 1.6. Image from Aharonian et al. (2008).

start operations in the next few years. The CTA will be the next level in TeV astronomy, being ten times more sensitive than the current generation of IACTs, and carrying enough power to significantly improve the results discussed in the next chapters of this thesis. 
Chapter 2

\section{Relevant sources and key science problems}

The $\gamma$-ray sky as observed by Fermi-LAT is filled with more than 5000 sources (Figure 2.1). In the Fermi-LAT fourth source catalog (4FGL; Abdollahi et al., 2020), these sources fall in several distinct classes, from AGN and normal galaxies to globular clusters and supernova remnants. But even with the improvements of Fermi-LAT with respect to EGRET, the poor angular resolution of the telescope imposes a challenge for source association. Indeed, $\sim 25 \%$ of the sources in 4FGL have no association with a low-energy counterpart.

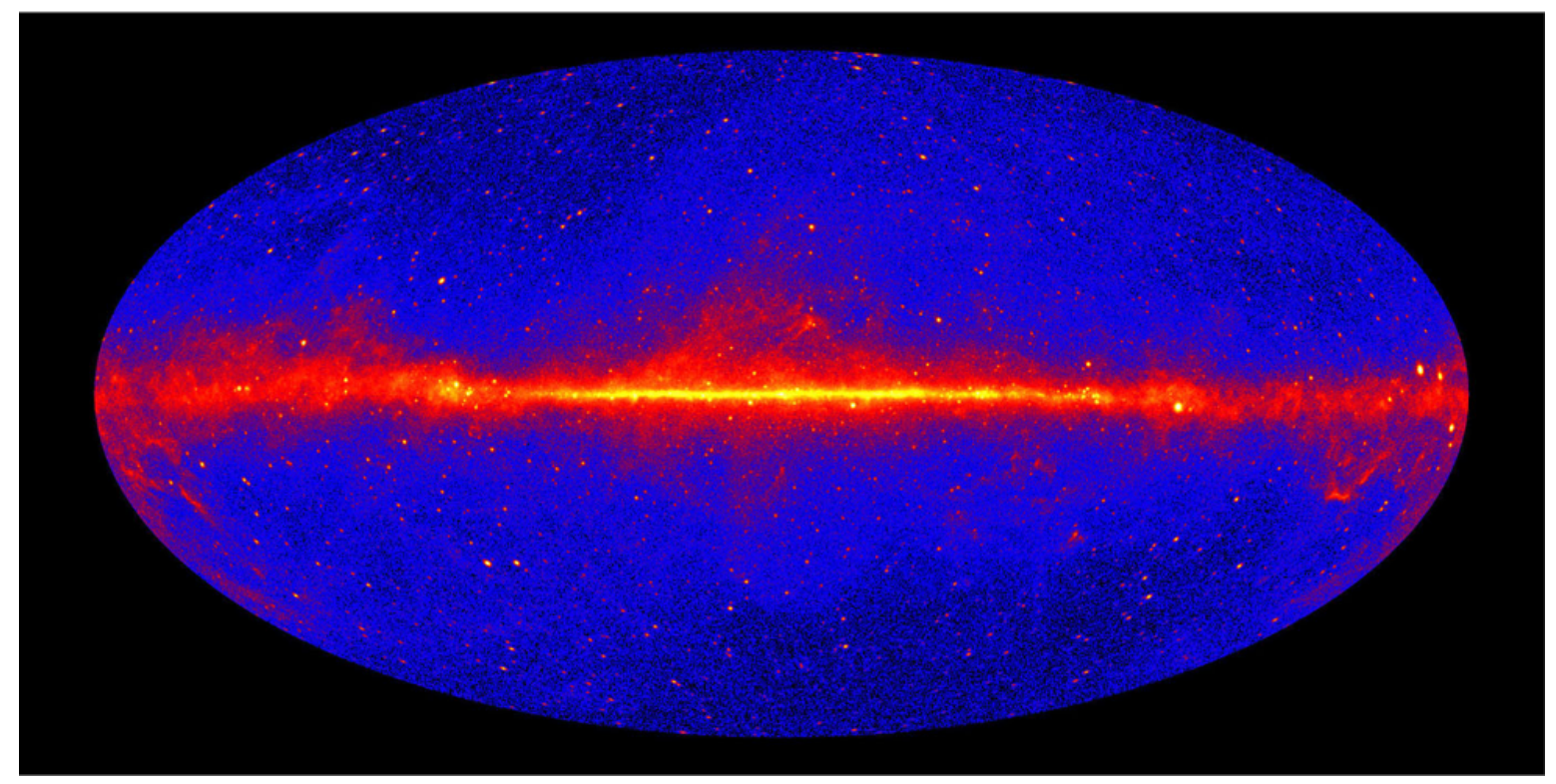

Figure 2.1: Fermi-LAT all sky survey in Galactic coordinates with photons collected over nine years of mission. Credit: The Fermi-LAT Collaboration, in public domain.

In this chapter we review the relevant source populations for the studies carried in this thesis. These are i) globular clusters, whose $\gamma$-ray emission is likely originated in the magnetospheres of the millisecond pulsars (MSPs) they usually host; ii) a subclass of AGN 
known as low-luminosity AGN (LLAGN), where the accretion is driven by hot accretion flows; and iii) the unassociated $\gamma$-ray sources (UGSs), which are expected to be mostly faint blazars. In the following sections, the key science problems are highlighted in italics.

\subsection{Globular clusters}

Globular clusters are evolved collections of stars, roughly spherical and tightly bounded by gravity (Figure 2.2). Given their high stellar densities, dynamical interactions between stars are a common phenomenon. The stellar flybys eventually produce close compact binaries in a mass-transferring regime, which can evolve in different ways depending on the mass of the companion (donor) star and the initial orbital period of the system. When the binary is formed by a neutron star and heavier companion, the mass transfer results in a common envelope evolution where the stars are subjected to drag forces from the envelope, shrinking the binary system up to the merger or until the envelope is ejected, which happens in timescales smaller than $10^{4}$ years (Tauris and Savonije, 1999). For a donor star lighter than the neutron star, the system becomes a low-mass X-ray binary (LMXB), which evolves on a stable and much longer timescale of $10^{7}-10^{9}$ years. These LMXBs are believed to be the progenitors of millisecond pulsars (MSPs), i.e., old "dead" pulsars spun up to millisecond periods by mass-accretion from a binary companion and hence "recycled" back to life. The formation rate of LMXBs in globular clusters is higher than anywhere else in the Milky Way and so is the presence of MSPs (Cheng et al., 2010).

A total of 31 globular clusters are listed in 4FGL (Abdollahi et al., 2020) and their $\gamma$-ray emission is commonly attributed to high energy processes occurring in the magnetospheres of MSPs (Abdo et al., 2010). This attribution has been further strengthened by the detection of pulsed $\gamma$-ray emission from the globular cluster NGC 6624 by Freire et al. (2011). On the other hand, folding the $\gamma$-ray data of the globular cluster 47 Tuc on known pulsar ephemerides reveals no significant pulsation, suggesting that the $\gamma$-ray emission in 47 Tuc is not due to a single MSP but rather from an entire population of MSPs, and this is likely the case for most of the $\gamma$-ray globular clusters. Understanding the physics of pulsars and MSPs is a fundamental step to better comprehend the high energy emission from globular clusters. 


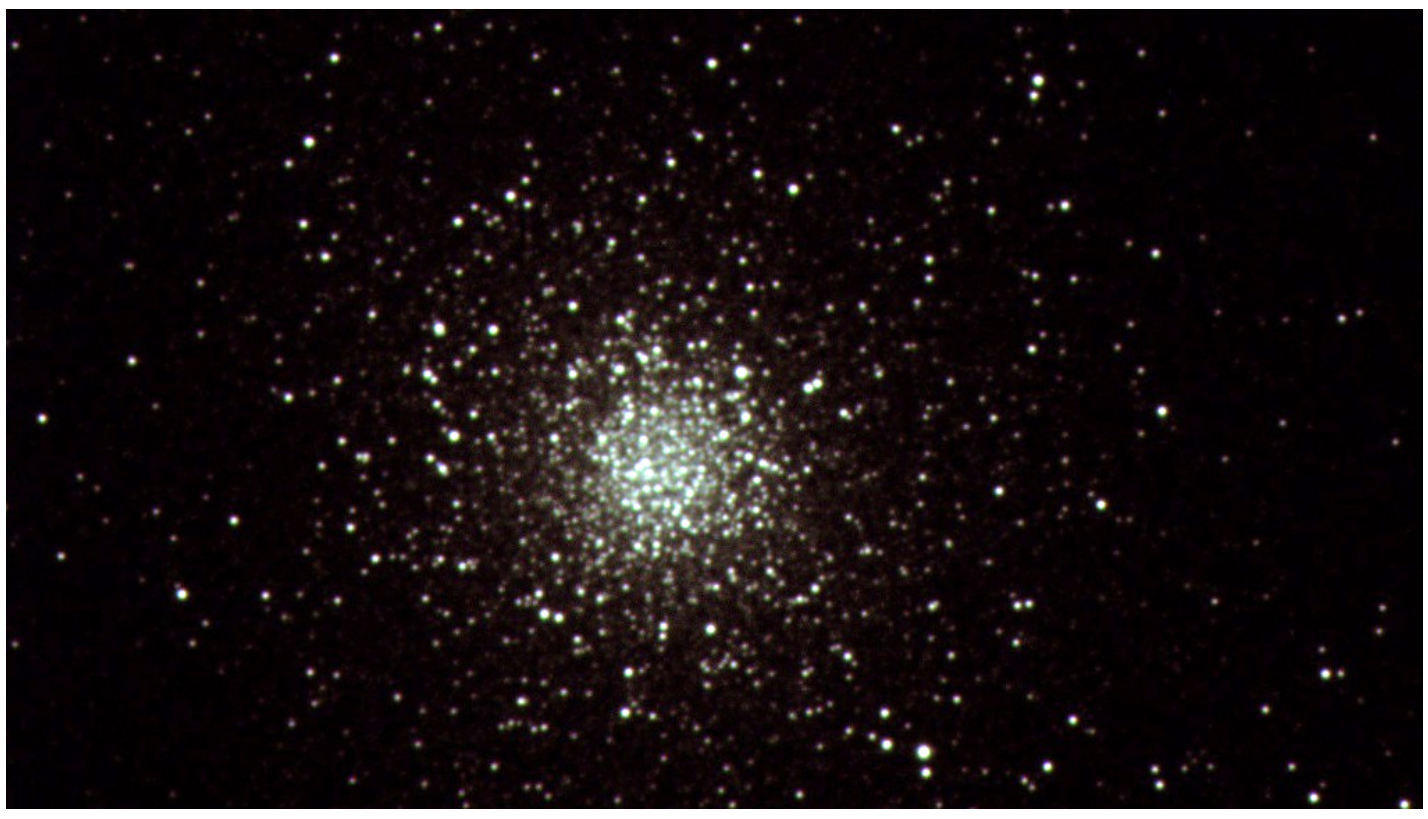

Figure 2.2: Messier 5, a typical globular cluster which is also detected in $\gamma$-rays by the Fermi-LAT. Image obtained by the author with the IAG $0.6 \mathrm{~m}$ Boller \& Chivens Telescope at the Observatório do Pico dos Dias, Brazil.

\subsubsection{Pulsars: back-of-the-envelope calculations}

The existence of neutron stars, audaciously proposed by Baade and Zwicky (1934), was serendipidously confirmed in 1967 when Hewish et al. (1968) observed the first radio pulsar, the PSR B1919+21. Pulsars are spinning highly magnetized neutron stars, where the spin axis is inclined with respect to the magnetic dipole axis and electromagnetic radiation is emitted out of its magnetospheric particle acceleration sites.

Neutron stars are forced by nature to be very dense and compact objects. We can establish a lower limit on the density $\rho$ of a gravitationally bound star by requiring that the centrifugal acceleration at its equator does not exceed the gravitational acceleration. Lets assume that a star of mass $M$ and radius $R$ rotates with angular velocity $\Omega=2 \pi / P$, where $P$ is the rotation period. Then:

$$
\begin{gathered}
\Omega^{2} R<\frac{G M}{R^{2}} \\
P^{2}>\frac{4 \pi R^{3}}{3} \frac{3 \pi}{G M},
\end{gathered}
$$

and by writing the mass in terms of the mean density $M=\rho\left(4 \pi R^{3} / 3\right)$, we have:

$$
\rho>\frac{3 \pi}{G P^{2}}
$$


which is a quite naive lower limit to $\rho$, but give us a fairly good result. For the fastest known pulsar, for instance, which has a period of $P=1.4 \times 10^{-3} \mathrm{~s}$, the minimum mean density is $10^{14} \mathrm{~g} \mathrm{~cm}^{-3}$, which is roughly the same density of nuclear matter.

For a star with mass greater than the Chandrasekhar limit

$$
M_{C h} \approx\left(\frac{h c}{2 \pi G}\right)^{3 / 2} \frac{1}{m_{p}^{2}} \approx 1.4 M_{\odot}
$$

the maximum radius is $R<(3 M / 4 \pi \rho)^{1 / 3}$, thus, in the case of the fastest pulsar, with $P=1.4 \times 10^{-3}$ s and $\rho>10^{14} \mathrm{~g} \mathrm{~cm}^{-3}$, we have $R \lesssim 20 \mathrm{~km}$. Stars less massive than $M_{C h}$ can survive as stable white dwarfs and are not considered here.

When the core of a star surpasses $M_{C h}$, it collapses from a radius $\sim 10^{11} \mathrm{~cm}$ to $\sim 10^{6} \mathrm{~cm}$, causing a reduction in surface area of $\sim 10^{10} \mathrm{~cm}^{2}$. As the core's magnetic flux $\Phi=\int \vec{B} \cdot \vec{n} d a$ must be conserved, the initial magnetic field then increases by the same factor of $\sim 10^{10}$, although in some cases a dynamo effect can intensify the magnetic field even more. The result is an extremely dense, highly magnetized star. The same logic above applies to the conservation of angular momentum, causing a boost on the spin of the collapsed core/star.

If the neutron star magnetic filed is approximately a dipole, any slight deviation angle $\alpha$ of the magnetic axis from the rotation axis will result in the emission of low-frequency electromagnetic radiation. The Larmor formula for the emitted radiation power from a rotating electric dipole is (Lorimer and Kramer, 2004):

$$
P_{r a d}=\frac{2 q^{2} a^{2}}{3 c^{3}}=\frac{2}{3} \frac{(q \ddot{r} \sin \alpha)^{2}}{c^{3}}=\frac{2}{3} \frac{\ddot{p}_{\perp}^{2}}{c^{3}}
$$

where $p_{\perp}$ is the perpendicular component of the electric dipole moment. Similarly, the power emitted by a magnetic dipole inclined with respect to the rotation axis is

$$
P_{\text {rad }}=\frac{2}{3} \frac{\ddot{m}_{\perp}^{2}}{c^{3}}
$$

where $m_{\perp}$ is the perpendicular component of the magnetic dipole moment. If we consider the neutron star as a uniformly magnetized sphere with radius $\mathrm{R}$ and surface magnetic field B, then $m=B R^{3}$ (Jackson, 1999), and for an inclined magnetic dipole rotating with angular velocity $\Omega$, we have

$$
m=m_{0} e^{-i \Omega t}
$$




$$
\begin{gathered}
\dot{m}=-i \Omega m_{0} e^{-i \Omega t} \\
\ddot{m}=\Omega^{2} m .
\end{gathered}
$$

Substituting this result into Equation 2.2 gives

$$
P_{\text {rad }}=\frac{2}{3} \frac{m_{\perp}^{2} \Omega^{4}}{c^{3}}=\frac{2}{3} \frac{m_{\perp}^{2}}{c^{3}}\left(\frac{2 \pi}{P}\right)^{4}=\frac{2}{3 c^{3}}\left(B R^{3} \sin \alpha\right)^{2}\left(\frac{2 \pi}{P}\right)^{4} .
$$

This electromagnetic radiation is emitted at the same frequency as the rotation of the star, thus being very low frequency radio waves, reaching $\lesssim 1 \mathrm{kHz}$ for the fastest known MSPs, so low that these radio waves cannot propagate through the ionized interstellar medium and hence cannot be directly observed (Lorimer and Kramer, 2004). The amount of energy emitted by this process is huge (see next paragraphs) and slows down the pulsar as it extracts the star's rotational kinetic energy (Ostriker and Gunn, 1969). In fact, the longwavelength radiation absorbed by the interstellar medium can light up a surrounding pulsar wind nebula, acting as a "megawave oven". The long-wavelength radio waves emitted by this process may not be confused with the much shorter wavelength radio pulses that we observe from the Earth.

We will come back to Equation 2.3 soon. By now this equation at least gives a hint that pulsars may gradually slow down due to radiation losses. This implies that the period derivative for a pulsar is $\dot{P}>0$ (note that $\dot{P}$ is dimensionless, i.e., seconds per second). From a purely mechanical perspective, if we can observe the period $\mathrm{P}$ and period derivative $\dot{P}$ (and indeed we can), then the rate at which the rotational energy $E_{\text {rot }}$ of a pulsar decreases is

$$
\frac{d E_{r o t}}{d t}=\frac{d}{d t}\left(\frac{1}{2} I \Omega^{2}\right)=I \Omega \dot{\Omega}
$$

where $I$ as the star's moment of inertia. As $\Omega=(2 \pi / P)$, its derivative on time is $\dot{\Omega}=$ $2 \pi\left(-P^{-2} \dot{P}\right)$. Substituting in the equation above:

$$
\frac{d E_{r o t}}{d t}=\frac{-4 \pi^{2} I \dot{P}}{P^{3}}
$$

By assuming a moment of inertia $I=\left(2 M R^{2}\right) / 5 \approx 10^{45} \mathrm{~g} \mathrm{~cm}^{2}$, with mass $M=1.4 M_{\odot}$ and radius $R=10 \mathrm{~km}$, the total emitted power for the Crab pulsar, which has $P=0.033$ 
s and $\dot{P}=10^{-12.4}$, is

$$
-P_{\text {rad }}=\frac{d E_{\text {rot }}}{d t} \approx-4 \times 10^{38} \mathrm{erg} \mathrm{s}^{-1}
$$

or $P_{\text {rad }} \approx 10^{5} L_{\odot}$ in very long-wavelength radiation $(\sim 30 \mathrm{~Hz})$. It greatly exceeds the average higher-frequency radio pulse luminosity from the Crab pulsar, $P_{\text {pulse }} \sim 10^{30} \mathrm{erg}$ $\mathrm{s}^{-1}$.

By putting Equations 2.3 and 2.4 together we have

$$
\begin{gathered}
P_{\text {rad }}=-\frac{d E_{\text {rot }}}{d t} \\
\frac{2}{3 c^{3}}\left(B R^{3} \sin \alpha\right)^{2}\left(\frac{2 \pi}{P}\right)^{4}=\frac{4 \pi^{2} I \dot{P}}{P^{3}} .
\end{gathered}
$$

By isolating $B$ and taking $0 \leq \sin ^{2} \alpha \leq 1$, we have

$$
B>\left(\frac{3 c^{3} I}{8 \pi^{2} R^{6}}\right)^{1 / 2}(P \dot{P})^{1 / 2} .
$$

Assuming $I \approx 10^{45} \mathrm{~g} \mathrm{~cm}^{2}$ and $R=10 \mathrm{~km}$ as above, the minimum magnetic field strength at the pulsar surface is

$$
B>3.2 \times 10^{19}(P \dot{P})^{1 / 2}
$$

which gives $B \gtrsim 4 \times 10^{12} \mathrm{G}$ for the surface magnetic field in the Crab pulsar.

We now have a simple way to estimate the magnetic field in the surface of a pulsar. As stated in Equation 2.6, we just need to measure the period $P$ and the time derivative of the period $\dot{P}$. Interestingly, the age of a pulsar can also be estimated based only on $P$ and $\dot{P}$. If we assume $B \sin \alpha$ as a constant in time in Equation 2.5 and isolate $P \dot{P}$, then

$$
P \dot{P}=P \frac{d P}{d t}=\frac{8 \pi^{2} R^{6}(B \sin \alpha)^{2}}{3 c^{3} I} \approx c t e
$$

and by integrating over the unknown pulsar lifetime $\tau$, we have

$$
\begin{aligned}
& \int_{P_{0}}^{P} P d P=c t e \int_{0}^{\tau} d t \\
& \frac{P^{2}-P_{0}^{2}}{2}=c t e \times \tau .
\end{aligned}
$$

By assuming $P_{0} \ll P$ and using the fact that $c t e=P \dot{P}$ (see Equation 2.7), we find the 
characteristic age of a pulsar

$$
\tau=\frac{P}{2 \dot{P}},
$$

which gives $\tau=1300$ years for the Crab pulsar (its actual age is $\approx 970$ years).

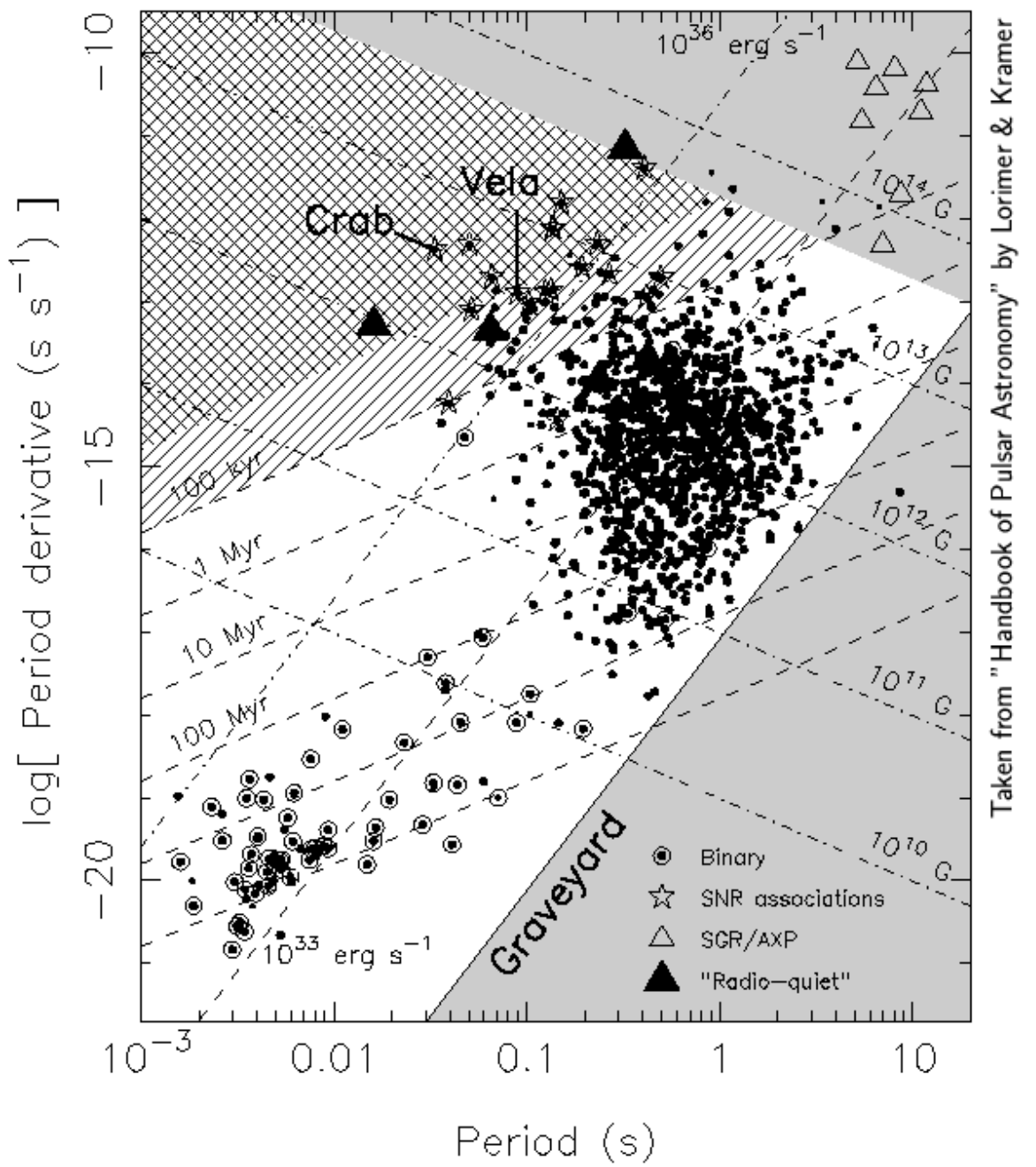

Figure 2.3: A $P-\dot{P}$ diagram. This diagram tracks the evolution of pulsars, playing a role similar to the Hertzsprung-Russell diagram for ordinary stars. It summarizes a huge amount of information based on only two observables, $P$ and $\dot{P}$. By measuring these parameters we can quickly estimate the age and magnetic field strength of a pulsar. See text for details. Image from (Lorimer and Kramer, 2004).

Equations 2.6 and 2.8 give $B$ and $\tau$ in terms of $P$ and $\dot{P}$, which are conveniently represented in the $P-\dot{P}$ diagram shown in Figure 2.3. This diagram is a powerful tool for following the evolution of pulsars, similar to the Hertzsprung-Russell diagram for ordinary stars. It offers the possibility to quickly estimate the pulsar's age, magnetic field strength, and even its spin-down power $\dot{E}$ (see Equation 2.4) based simply on the two observables $P$ and $\dot{P}$. Pulsars are born in supernovae explosions and appear in the upper left corner of the diagram. If $B$ is constant in time and pulsars age roughly as described above, they 
gradually move to the right and down, along lines of constant $B$ and crossing lines of constant characteristic age $\tau$.

Young pulsars $\left(\tau<10^{5}\right.$ years) are often found inside or on the neighborhood of supernova remnants, while older pulsars are not, either because their supernova remnants have faded away or due to the expel of the pulsar by an asymmetric supernova explosion (also known as pulsar kick; see Hansen and Phinney, 1997, for more details). As pulsars age, they move across the graveyard line, below which they cease to emit radio pulses. Basically all short-period pulsars (bottom left corner of the diagram) are in binary systems. These pulsars have been spun up to millisecond periods by accreting mass and angular momentum from their companions up to the point where they emit radio pulses again. The mass accreted by the neutron star in the recycling process reduces the pulsar magnetic field by a factor as high as $\sim 1000$, causing a relatively small spin-down rate (Urpin and Geppert, 1995; Cheng and Zhang, 1998), and hence making the pulsar luminous for as long as billions of years.

\subsubsection{Magnetospheric gamma-rays}

There are three major models explaining the $\gamma$-ray emission from MSPs. In the polar cap model, charged particles are accelerated by strong electric fields induced by the rotating magnetic field in the star's polar cap (Sturrock, 1971). These particles move along magnetic field lines and produce curvature radiation up to the GeV energy range. Photons with energies above $\sim 1 \mathrm{GeV}$ quickly interact with the thermal X-rays from the black body radiation in the surface of the neutron star and with the polar magnetic field, hence producing $e^{+}+e^{-}$pairs and shaping the predicted $\gamma$-ray spectrum as a super-exponential cutoff curve.

In the slot gap (Arons, 1983) and outer gap (Cheng et al., 1986) models, particle acceleration takes place along vacuum regions (i.e., no magnetic field lines) in the pulsar's magnetosphere, both far from the neutron star surface, as shown in Figure 2.4. In the case of the outer gap model, the gap is limited by the light cylinder, a virtual cylindrical surface of radius $R_{L C}>>R_{\text {star }}$ up to which the plasma frozen to the magnetic field lines can corotate with the neutron star at speeds $\Omega \times R_{L C}<c$. The $\gamma$-rays from both models are produced mainly by curvature radiation.

The $\gamma$-ray pulsar spectra observed by Fermi-LAT are commonly well described by a 


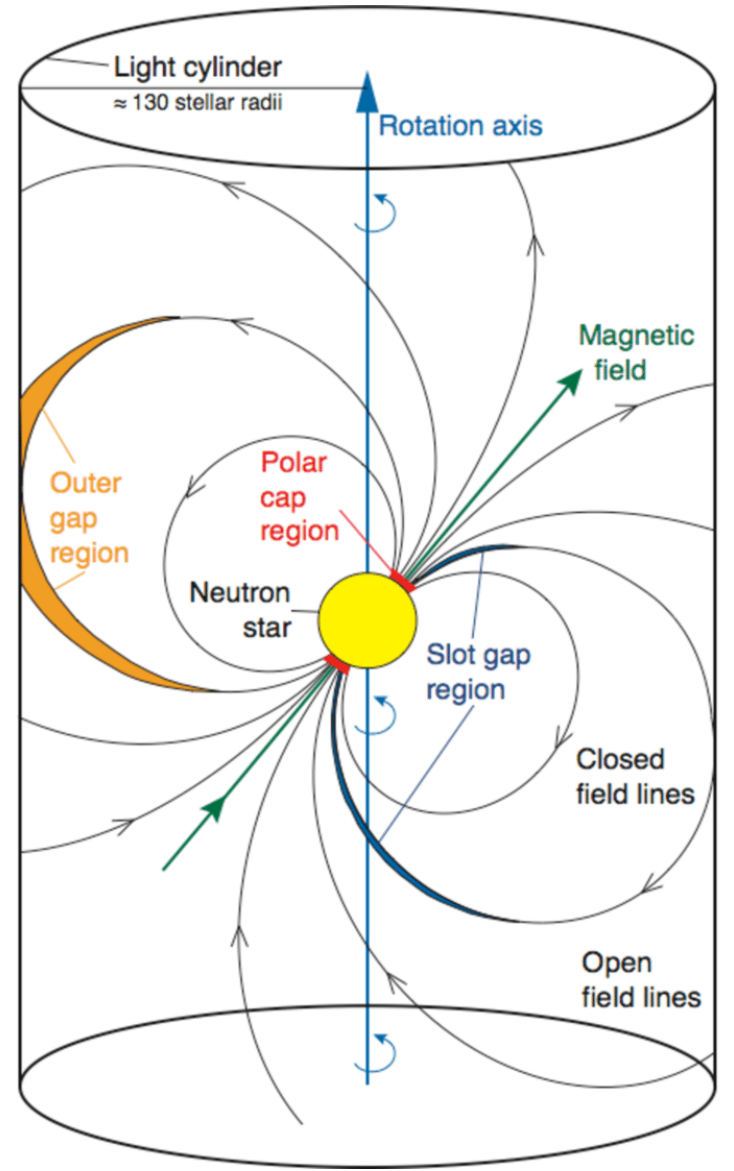

Figure 2.4: A sketch of the Crab pulsar magnetospheric emission sites. The polar cap, slot gap and outer gap emission sites are indicated in colors. Image from Aliu et al. (2008).

power law with an exponential cutoff in the 1-5 GeV energy range, disfavoring the superexponential cutoff predicted by the polar cap model and favoring the slot and outer gap models (Caraveo, 2014). The slot gap scenario, however, needs a boost in efficiency to account for the observed set of Fermi-LAT detections (Caraveo, 2014). The outer gap model is then the best model to date, although it is not perfect and, in a few cases, the pulsars are better modelled by the other two scenarios (Romani and Watters, 2010). All these models, however, share the common emission mechanism of curvature radiation, which will be discussed in Chapter 3 .

\subsubsection{Dynamical formation and evolution of MSPS}

Unlike the pulsars found anywhere else in the Galaxy, those found in globular clusters are mostly MSPs. As MSPs are likely the progeny of LMXBs, and the total number of LMXBs in a globular cluster scales with the stellar encounter rate (Pooley et al., 2003), it is reasonable to assume that the number of MSPs per globular cluster scales in a similar 
way. Observations in the Fermi-LAT energy band are ideal for testing such a correlation, as $\gamma$-rays are not affected by dispersion and scattering by the turbulent ionized interstellar medium (as is the case for radio waves), neither by interstellar absorption (like X-rays and UV; Fireman, 1974).

The encounter rate comes down to bringing two stars close together, hence depending on the square of the clusters' stellar number density $n$. The faster the stars in a globular cluster, the weaker are the one-to-one interactions, and thus the overall encounter rate may be somehow inversely proportional to the stellar velocity dispersion $\sigma$. By integrating over the cluster's radius, we write the two-body stellar encounter rate as $\Gamma \propto \int\left(n^{2} / \sigma\right) 4 \pi r^{2} d r$ (Bahramian et al., 2013).

Stellar distributions in globular clusters are well modelled by truncated isothermal spheres known as King models (King, 1962, 1966). These models have a core region of nearly constant stellar density followed by an abrupt density cutoff outside the core. To simplify the computation of the encounter rate, several works have approximated the total $\Gamma$ of a cluster by only considering the averaged $\Gamma$ in the nearly constant density core (see e.g. Abdo et al., 2010; Hui et al., 2010) with radius $r_{c}$. In some cases, when $\sigma$ is not well known for the cluster, we can use $\sigma \propto n^{0.5} r_{c}$ to simplify the encounter rate to $\Gamma \propto n^{1.5} r_{c}^{2}$ (Verbunt and Hut, 1987).

There is a hypothesis (Verbunt and Freire, 2014) that the lifetime of a binary system within a globular cluster depends on another dynamical parameter: the encounter rate per formed binary $\Lambda \propto \sqrt{n} / r_{c}$. The argument is that for a cluster with specific dynamical properties, the slow and stable evolution of LMXBs may be interrupted before the formation of MSPs, in a timescale $\tau=1 / \Lambda$. This translates into the probability that a formed binary will go through a subsequent encounter, close enough to disrupt the binary or in some cases exchange binary members (Verbunt and Freire, 2014). However, this influence of $\Lambda$ on the formation of MSPs has never been tested before. In this thesis we test this hypothesis by assessing the dependence of the total number of $\gamma$-ray MSPs per globular cluster as a function of $\Lambda$. The details on our results can be found in Chapter 4 . 


\subsection{Low-luminosity active galactic nuclei (LLAGNs)}

The gravitational potential energy released by the accretion of matter onto a black hole is the primary source of power in AGNs. A reliable model describing the accretion physics was first developed by Shakura and Sunyaev (1973) and Lynden-Bell and Pringle (1974). The gas temperature in this model depends on the mass of the central object and ranges from $10^{4}$ to $10^{7} \mathrm{~K}$, which is cold if we compare to the virial temperature of the system. The disk is geometrically thin and optically thick (commonly referred as Shakura-Sunyaev disk), with an expected spectrum of integrated black-body radiation, and can be applied when the mass accretion rate $\dot{m}$ lies in the range $0.01 \dot{m}_{E d d}<\dot{m}<\dot{m}_{E d d}$, where $\dot{m}_{E d d}$ is the Eddington accretion rate defined by $\dot{m}_{E d d}=10 L_{E d d} / c^{2}=1.39 \times 10^{18}\left(M / M_{\odot}\right) \mathrm{g} \mathrm{s}^{-1}$, with $M$ being the mass of the central object and assuming an accretion efficiency of $10 \%$.

Several AGNs and stellar black hole binaries have been successfully modeled by thin disks (see e.g. Koratkar and Blaes, 1999; McClintock et al., 2013), but there are other possibilities for accretion in astrophysics. For instance, when $\dot{m}$ approaches or exceeds $\dot{m}_{E d d}$, the optical depth of the infalling gas become too high to allow the produced radiation to escape. The radiation is then trapped in the gas and advected towards the central object, lowering the radiative efficiency of the accretion and consequently decreasing the system's luminosity. This kind of disk is known as slim disk (Ohsuga et al., 2005).

Another possibility for the accretion scenario is the hot accretion flow model (Yuan and Narayan, 2014), where the accretion rate is very low and the low-density accreted gas is optically thin. One of the main characteristics of this model is the introduction of a twotemperature plasma, where the ions are two or three orders of magnitude hotter than the electrons and reach temperatures similar to the virial temperature. The two-temperature nature of the plasma in a hot accretion flow is not simply an assumption, but a consequence of the model. As the electrons radiate much more efficiently than ions, their temperature drops faster. Furthermore, due to the low gas densities expected in hot flows, Coulomb collisions are not that common, hence quenching the transfer of energy between ions and electrons. AGNs hosting hot accretion flows are termed LLAGNs.

We should avoid thinking of LLAGNs as scaled-down versions of quasars and Seyferts. LLAGNs have several characteristics which cannot be explained by a geometrically thin, optically thick disk. They also account for the majority of the nuclear activity in nearby 
galaxies (Ho, 2008), and the matter around their central black holes are likely in the radiatively inefficient accretion flow (RIAF) mode.

\subsubsection{Radiatively inefficient accretion flow (RIAF)}

When the luminosity of the system is $L \ll 0.1 \dot{m} c^{2}$, the gas in the surroundings of a black hole is reasonably described by a RIAF model (Narayan and Yi, 1994a,b). The released gravitational potential energy in the accretion flow is trapped in the gas and boosts the thermal energy of the system to a level comparable to its gravitational potential energy. Close to the black hole (at a few Schwarzschild radii), this implies

$$
\left(\frac{G M m_{p}}{r}\right)\left(\frac{2}{3 k T}\right) \sim 1
$$

or

$$
T \sim\left(\frac{2 G M m_{p}}{3 k r}\right) \sim 0.1 m_{p} c^{2} / k \sim 10^{12} K,
$$

for the ions, and $T \sim 5 \times 10^{9} \mathrm{~K}$ for electrons. Note that because collisions are unimportant, there is no strong reason to assume a thermal electron distribution function, although this possibility is often explored in the literature (Quataert, 2003; Rieger, 2011). The absence of collisions implies in a critical accretion rate $\dot{m} \sim 0.05 \dot{m}_{E d d}$ above which RIAFs cannot exist.

There is a simplified case of a RIAF known as advection-dominated accretion flow (ADAF), which predicts that the structure of the flow is similar to a spherically symmetric accretion (i.e., Bondi accretion; Bondi, 1952), even after accounting for angular momentum and viscosity (Narayan and Yi, 1994a). In ADAF models the gas rotates at $\Omega \approx(0.3-$ $0.5) \Omega_{K}$, where $\Omega_{K}=\sqrt{G M / R^{3}}$ is the Keplerian angular velocity. The sub-Keplerian rotation is a consequence of the large internal pressure support, which also inflates the disk to a geometrically thick profile with scale height $H \sim R$ (Quataert, 2003). Note that advection dominated accretion is not synonymous with hot accretion. For instance, the slim disk is advection dominated, although for a very different reason (long radiative diffusion time) compared with the hot ADAF (long cooling time).

In contrast with Shakura-Sunyaev disks where $L \propto \dot{m}$, the total radiative luminosity of an ADAF goes with the square of the mass accretion rate (Mahadevan, 1997). For a 


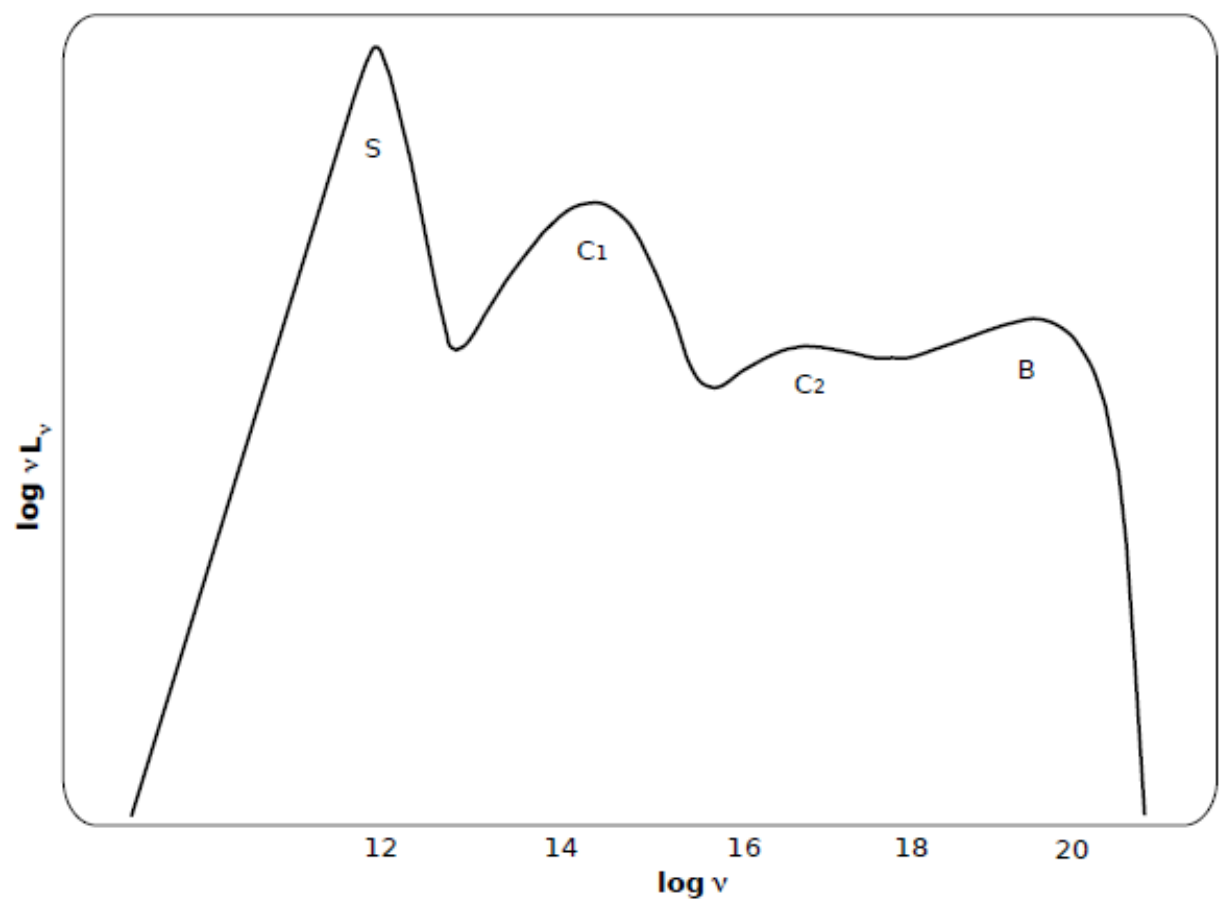

Figure 2.5: Outline of a pure ADAF spectrum for a supermassive black hole. Relativistic electrons in the flow produce a synchrotron peak $(\mathrm{S})$ around $10^{12} \mathrm{~Hz}$. At low accretion rates, multiple Compton peaks are formed $(\mathrm{C} 1=$ Compton scattered, $\mathrm{C} 2=$ twice Compton scattered), and the hard X-ray/soft $\gamma$-ray band is dominated by bremsstrahlung emission (B). Image modified from Rieger (2011).

viscosity parameter $\alpha$, the luminosity is

$$
L_{A D A F} \sim \frac{2 \times 10^{-2}}{\alpha^{2}}\left(\frac{\dot{m}}{\dot{m}_{E d d}}\right) \dot{m} c^{2} \propto \dot{m}^{2}
$$

thus being much smaller than the luminosity expected from a Shakura-Sunyaev disk.

The spectral energy distribution (SED) generated by a pure ADAF model is shown in Figure 2.5. The emission ranges from radio up to hard X-rays, where the synchrotron radio emission at the highest peak on the plot (tagged by "s") comes from the innermost part of the disk:

$$
\nu_{p}(r)=10^{12}\left(\frac{10^{8} M_{\odot}}{M}\right)^{1 / 2}\left(\frac{\dot{m}}{0.01 \dot{m}_{E d d}}\right)^{1 / 2}\left(\frac{T_{e}}{10^{9} K}\right)^{2}\left(\frac{r_{s}}{r}\right)^{5 / 4} \mathrm{~Hz},
$$

while the lower frequency synchrotron emission originates from further out (Rieger, 2011). Compton upscattering of the low-energy photons by relativistic electrons can occur more than once, forming multiple inverse Compton peaks extending up to X-rays. As we decrease $\dot{m}$, the inverse Compton scattering becomes less effective, and the X-ray spectrum is dominated by bremsstrahlung emission peaking at $h \nu \sim k T_{e}$, and with luminosity scaling 


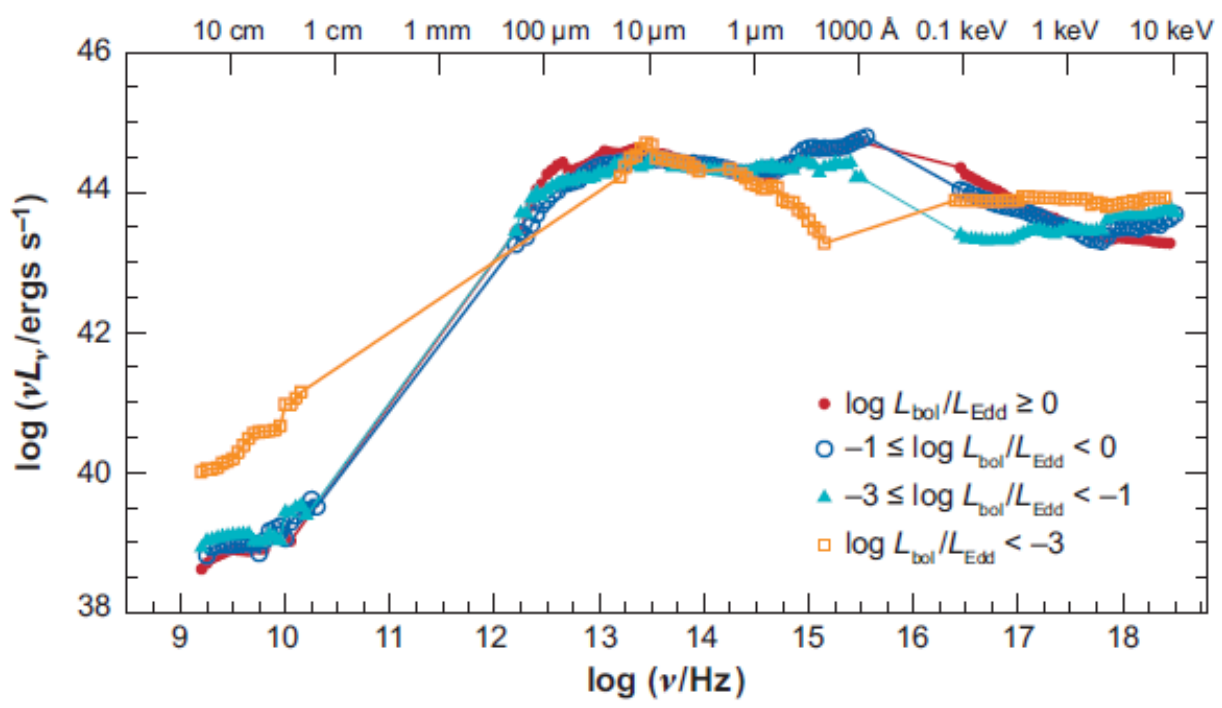

Figure 2.6: Average SEDs for AGN populations binned by Eddington ratio. The SEDs are normalized at $1 \mu \mathrm{m}$. We see that LLAGNs are usually radio loud and lack a big blue bump. Image from Ho (2008).

as $L_{\text {brems }} \propto \dot{m}^{2}$.

Given the high pressure, $p$, and low density, $\rho$, environment in an ADAF, the isothermal sound speed $\left(c_{s}=\sqrt{p / \rho}\right)$ of the system is comparable to the escape speed of the black hole surroundings, therefore making this model prone to developing strong outflows and, by extension, jets (Quataert, 2003). Indeed, the observational evidence indicates that essentially all LLAGNs are radio loud (Ho, 2008). In contrast, jets tend to be weaker and often missing in systems with cool geometrically thin disks (Yuan and Narayan, 2014). In Figure 2.6 we show the normalized and averaged SEDs of AGN populations in different accretion scenarios and two features pop up instantly: i) LLAGN are radio loud, where they generally exceed the radio-to-optical luminosity ratio $L_{\nu}(5 \mathrm{GHz}) / L_{\nu}(B)=10$, and ii) they lack the "big blue bump" characteristic of the integrated black-body radiation of Shakura-Sunyaev systems.

Besides the possibility of $\gamma$-ray emission via inverse Compton in the jets of LLAGNs, the hot accretion flow is predicted (Mahadevan et al., 1997) to host the necessary conditions for proton-proton collisions and hence pion generation (Figure 2.7). If this prediction holds, the pion decay should generate $\gamma$-rays by different channels as already explained in §1.2.2, which in principle are within the energy range of Fermi-LAT (see §3 for details in the physics of pion-generated $\gamma$-rays). This prediction, however, has never been tested before. Therefore, in this thesis we use Fermi-LAT observations to test this hypothesis and probe the origin of $\gamma$-rays in LLAGNs. A detailed discussion of our results can be found in 


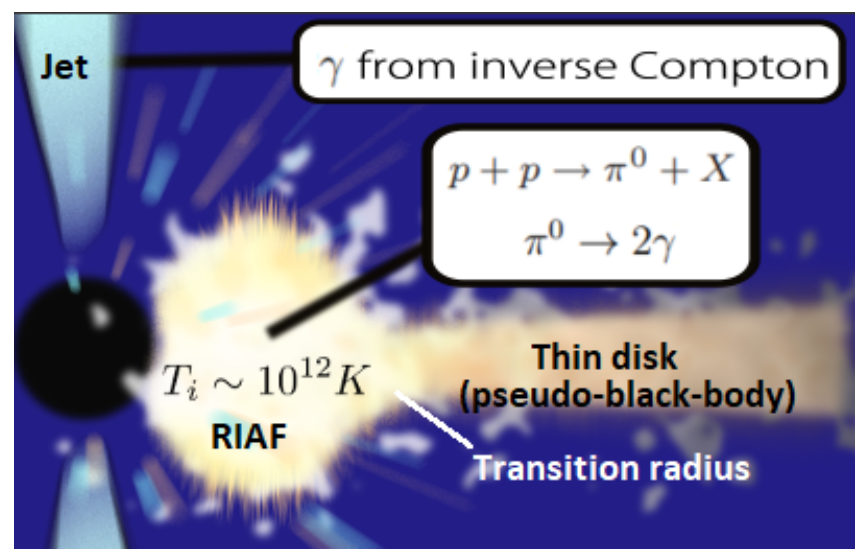

Figure 2.7: Illustration of a RIAF system highlighting two channels of $\gamma$-ray production.

Chapter 5 .

\subsection{Unassociated gamma-ray sources (UGSs)}

Finding the low-energy counterparts of $\gamma$-ray sources is challenging. As explained in $\S 1.2 .1, \gamma$-rays cannot be reflected or refracted. The detector is then limited to reconstruct the arrival direction of a photon based on the induced particle shower, unavoidably limiting the telescope's angular resolution. The positional uncertainties of the $\gamma$-ray sources can then be orders of magnitude larger than those obtained in lower energy bands, compromising the association by positional coincidence on the sky in several cases. The typical $\gamma$-ray positional uncertainty in the 4FGL is $4^{\prime} \sim 5^{\prime}$ at the $95 \%$ confidence level, but can reach $\sim 1^{\circ}$ in some cases (Abdollahi et al., 2020).

The number of potential counterparts lying within the error ellipse of a $\gamma$-ray source can be huge, as shown in Figure 2.8, and generally increases towards the Galactic plane, where we have a larger density of background sources and a limited knowledge of the Galactic $\gamma$-ray background. This problem, however, is attenuated by the fact that any $\gamma$-ray source must be dominated by non-thermal radiation (Massaro et al., 2016). Normal stars and thermal X-ray objects, for instance, are not plausible candidates as counterparts of 4FGL sources, implying that relatively few astrophysical objects are expected to be $\gamma$-ray emitters. Exceptions to the rule are the Sun and the Moon, which are visible by Fermi-LAT due to their proximity and production of $\gamma$-rays via interactions of cosmic rays with the solar wind/lunar surface.

Since the CGRO era, the association of $\gamma$-ray sources to their counterparts is based on 


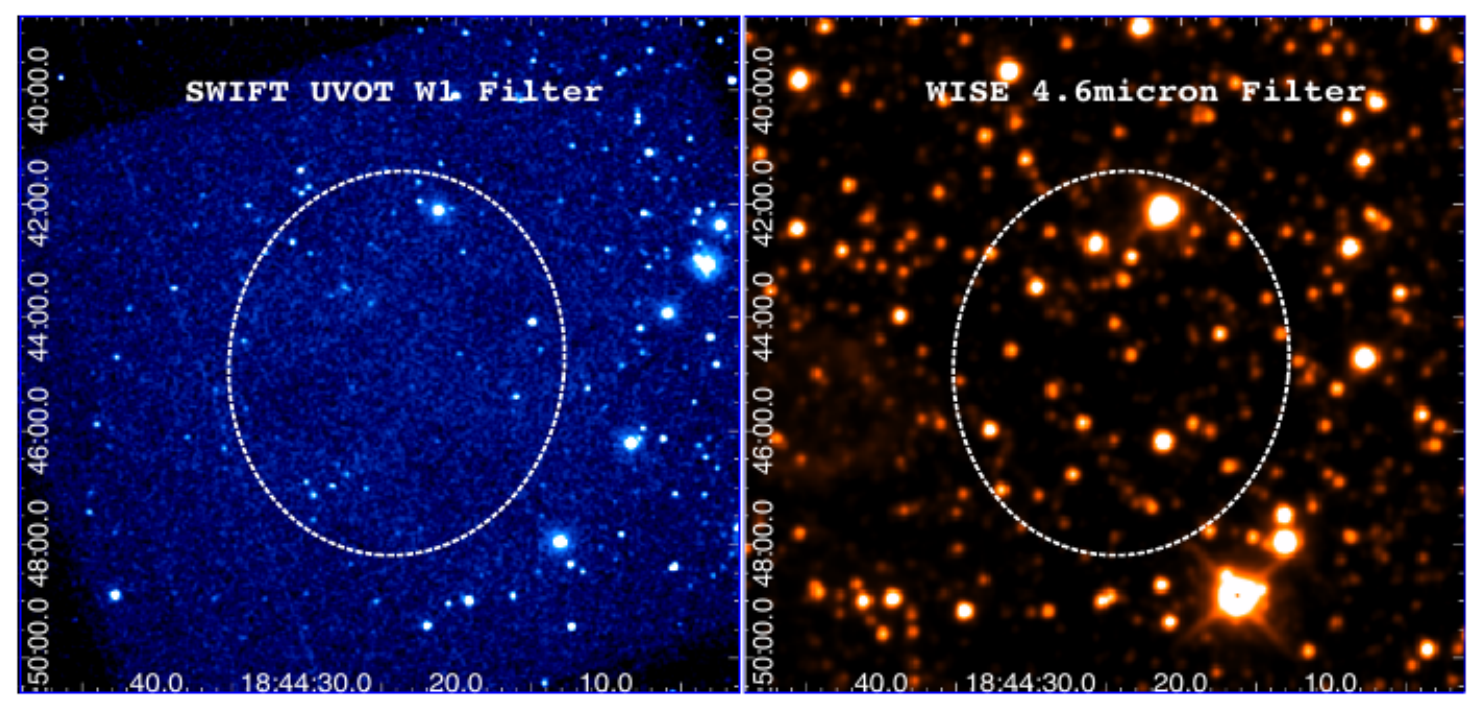

Figure 2.8: Ultraviolet (left) and mid-infrared (right) images overlaid with a typical $\gamma$-ray source error ellipse (at 95\% confidence level). Not all of these point sources are good $\gamma$-ray counterpart candidates, as plenty of them may be dominated by a thermal spectrum. Image from Massaro et al. (2016).

statistical methods (Mattox et al., 1997). These methods assign a value for the probability that the low-energy source is the real counterpart for a given $\gamma$-ray object. In the FermiLAT catalogs, only high-confidence counterpart candidates ( $>80 \%$ association probability) are selected as associations. Of course some real associations will be missed and some false associations will be taken as correct, however, in general, these associations provide a welldefined sample of $\gamma$-ray sources that can be used for population studies. An extra and rare problem occurs when two or more counterpart candidates belonging to known classes of $\gamma$-ray emitters lie within a single positional uncertainty region of a $\gamma$-ray source. In these few cases (nearly a handful) the $\gamma$-ray emission could be due to one of them or both.

The methods commonly used in the Fermi-LAT catalogs are i) the Bayesian method, where the association probability (AP) is assessed by taking into account the counterpart candidate background densities and angular separation between the center of the $\gamma$-ray source and the candidate counterpart, ii) the likelihood ratio method, where the AP is computed based on angular separation and integrated flux, and iii) the $\log N-\log S$ approach, which is a modified version of the Bayesian method used in the Fermi-LAT AGN catalogs (Ackermann et al., 2011).

All these association methods rely on angular separation and sometimes on integrated flux and background density, thus not taking into account intrinsic physical properties, as luminosity or spectral shape, which could reflect the nature of the candidate counterpart when computing the AP. In this thesis, we propose a couple of alternative association 


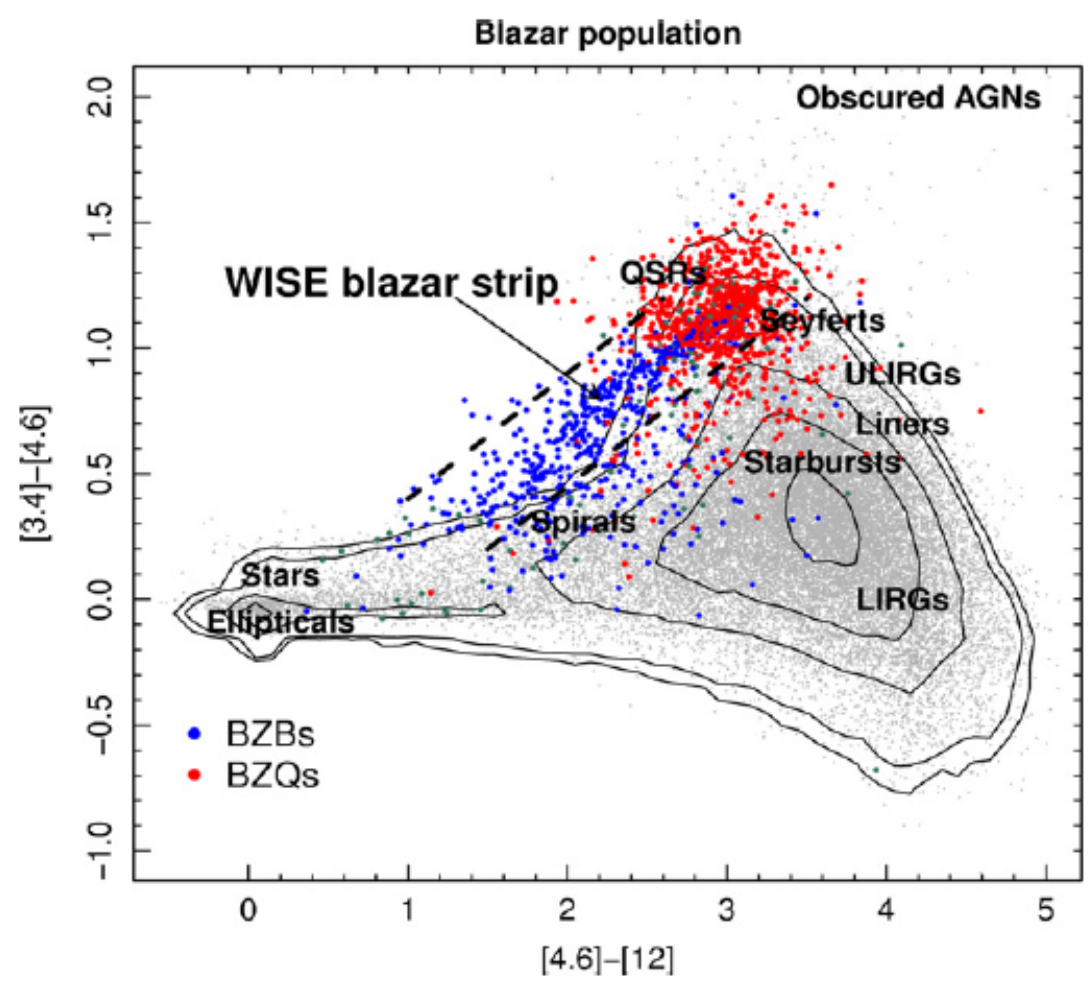

Figure 2.9: The [3.4]-[4.6] $\times$ [4.6]-[12] $\mu \mathrm{m}$ MIR color-color diagram of WISE sources. The blazars, divided into flat spectrum radio quasars (red) and BL Lacs (blue), occupy a very distinct region of the diagram, the so called blazar strip, due to their non-thermal emission. Thermal sources are represented as grey dots. Image modified from Massaro et al. (2011a).

methods which assess the AP of a given counterpart based on its mid-infrared (MIR) colors and radio-loudness. No angular separation or integrated flux are required, hence making our methods mainly independent of non-physical parameters. Our approach starts by testing the efficiency of the Wide-field Infrared Survey Explorer (WISE) Blazar-like Radio-Loud Sources (WIBRaLS; D'Abrusco et al., 2019) catalog in spotting the MIR counterparts of $\gamma$-ray blazars, and then we use these results in a modified version of the likelihood ratio method and in a random forest algorithm. Our methods, however, are only applicable to the association of $\gamma$-ray blazars, as they have very characteristic infrared colors (see Figure 2.9), and must be modified for different classes of $\gamma$-ray emitters. A detailed discussion of these results can be found in Chapters 6 and 7 .

Going further in this topic, we worked on an optical spectroscopic follow-up campaign to directly spot $\gamma$-ray counterpart candidates for UGSs and unveil the nature of blazar candidates of uncertain type (BCUs), which are the counterparts of $\gamma$-ray sources having blazar-like SEDs but with no optical spectra available on literature. The observations we carried out are part of a long-standing campaign responsible for significantly increasing 
the number of associated $\gamma$-ray sources (Massaro et al., 2013; Paggi et al., 2014; Landoni et al., 2015a; Massaro et al., 2015; Ricci et al., 2015; Crespo et al., 2016). Our searches are focused on blazars, and indeed most of the UGSs are expected to be blazars, due to their almost uniform sky distribution (indicating that they are potentially extragalactic sources). A detailed discussion of our results can be found in Chapter 8. 
Chapter 3

\section{Physics of gamma-ray emission}

For the source populations relevant to this work, the $\gamma$-rays are produced mainly by nonthermal processes located deep into extreme astrophysical environments and controlled by a compact object like a neutron star or black hole. In this chapter we summarize the main radiative processes driving the $\gamma$-ray emission and attenuation in the sources relevant to this thesis. We start by reviewing (i) the curvature radiation emitted by charged particles accelerating in open magnetic field lines in the magnetospheres of pulsars, (ii) the decay of neutral pions in a hot RIAF, (iii) the inverse Compton scattering of soft photons to $\gamma$-ray energies in AGN jets, and (iv) the $\gamma-\gamma$ absorption in environments with a high density of soft photons.

\subsection{Outer gap acceleration and curvature radiation}

In $\gamma$-ray emission models for pulsars, $e^{+}+e^{-}$pairs are accelerated by rotation-induced electric fields $E_{\|}$parallel to the open and curved magnetic field lines of the neutron star (Hirotani, 2008b). The acceleration resulting from the curvature stimulates the emission of curvature radiation by these charged particles, which is strongly polarized parallel to the plane of curvature (exactly the opposite of synchrotron radiation).

For spinning compact objects embedded in a magnetic field, several mechanisms would sustain enough $e^{+}+e^{-}$pair production to shield $E_{\|}$, except in a region close to the border of the light cylinder (radius $=R_{L C}=c / \Omega$ ) known as outer gap (Cheng et al., 1986). The

outer gap size is inward limited by the null surface $\vec{\Omega} \cdot \vec{B}=0$ (i.e., where the magnetic field lines are perpendicular to the star's rotation axis) and outward limited by the light cylinder, as shown in Figure 3.1. The maximum electric potential drop across the gap is 


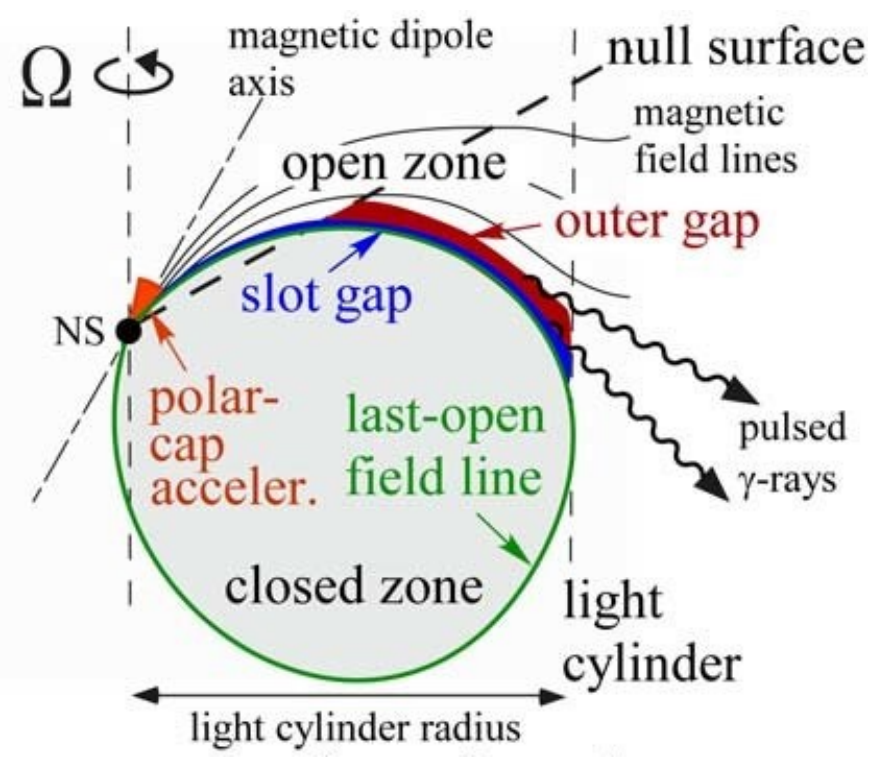

Figure 3.1: A slice of the pulsar magnetosphere. The outer gap is assumed to be located between the null charge surface and the light cylinder. Image modified from Hirotani (2008a).

(in statvolts):

$$
\Delta V=2 \times 10^{15}\left(\frac{\mu}{0.1}\right)\left(\frac{\Phi_{B}}{10^{22} \mathrm{G} \mathrm{cm}^{2}}\right)\left(\frac{\Omega}{100 \mathrm{rad} \mathrm{s}^{-1}}\right)\left(\frac{10^{8} \mathrm{~cm}}{R_{c}}\right)\left(\frac{L}{10^{8} \mathrm{~cm}}\right) \mathrm{V}
$$

where $\mu$ is the ratio between the vertical width and azimuthal breadth of the gap, assumed to be constant over the gap. The nearly constant magnetic flux through the gap is represented by $\Phi_{B}$, and the neutron star angular velocity is $\Omega$. The parameter $R_{c}$ is the curvature radius of the magnetic field lines and $L$ is the total length of the gap (Cheng et al., 1986).

In the case of synchrotron radiation, relativistic charged particles move around an arc or circle perpendicular to the magnetic field lines, while in curvature radiation the charged particles are frozen to the strong magnetic field and accelerated by the electric potential given in Equation 3.1 along the open magnetic field lines, such that the actual acceleration corresponds to the centripetal one, i.e., with curvature radius

$$
R_{c}=\frac{\gamma m_{0} c^{2}}{Z e B \sin \theta}
$$

where $\gamma m_{0} c^{2}$ is the total energy of the accelerated particle, $Z$ is the charge number, $e$ is the electron's charge and $B \sin \theta$ is the perpendicular component of the magnetic field with respect to the rotation axis. 
The curvature radius from Equation 3.2 defines the critical frequency $\nu_{c} \approx 3 c \gamma^{3} / 4 \pi R_{c}$ where most of the radiation is emitted. Just like synchrotron emission, the spectrum produced by curvature radiation is a power law $I(\nu) \propto \nu^{1 / 3}$ for $\nu \ll \nu_{c}$ with an exponential decay for $\nu \gg \nu_{c}$. The peak of the emission, however, is shifted towards the $\gamma$-ray band of the electromagnetic spectrum, and the total radiated power by a single charged particle follows (Rieger, 2011)

$$
P_{c}=\frac{2 Z^{2} e^{2} c}{3 R_{c}^{2}} \gamma^{4}
$$

thus indicating a larger emitted power for a stronger magnetic field (as $R_{c} \propto B^{-1}$ in Equation 3.2). Radiative losses in curvature radiation are supposed to produce a cutoff in the spectrum above energies of $\epsilon_{c u t}=150 \times(E / B)^{3 / 4} \sqrt{R / R_{L C}} \mathrm{GeV}$, where the electric field $E \lesssim 0.1 B$ in pulsar gaps, and $R$ needs to be at least $\gtrsim 0.1 R_{L C}$ to avoid $\gamma-\gamma$ absorption (see $\S 3.4)$ near the pulsar surface (Bühler and Blandford, 2014). The expected cutoff energy for the values above is then $\epsilon_{\text {cut }} \approx 8 \mathrm{GeV}$.

\subsection{Pion decay in hot accretion flows}

From radio up to X-rays, the spectrum of a RIAF is determined by considering only the cooling processes of the electrons, i.e., synchrotron, bremsstrahlung and inverse Compton scattering. For the $\gamma$-ray emission, however, the electrons play no significant role. Indeed, as shown in $\S 2.2$, the ion temperature in a RIAF can be as high as $T_{i} \sim 10^{12} \mathrm{~K}$, and $\gamma$-rays are expected to be produced through the decay of neutral pions, $\pi^{0}$, created in proton-proton collisions (Mahadevan et al., 1997):

$$
p+p \rightarrow p+p+\pi^{0}, \quad \pi^{0} \rightarrow \gamma+\gamma
$$

The process of $\pi^{0}$ decay generates a pair of $\gamma$-rays in $\approx 99 \%$ of the cases, providing the main channel for ion cooling in a RIAF. For $\pi^{0}$ production to become kinematically possible, the kinetic energy of the bombarding proton must exceed $E_{t h} \sim 280 \mathrm{MeV}$, which is above the rest energy of the $\pi^{0}$ (i.e., $135 \mathrm{MeV}$ ). This is required because, in the end of the interaction, there is still considerable kinetic energy in the remaining protons from the original proton-proton collision (Rieger, 2011).

Once the radio-to-X-rays RIAF spectrum due to the cooling processes of electrons is 


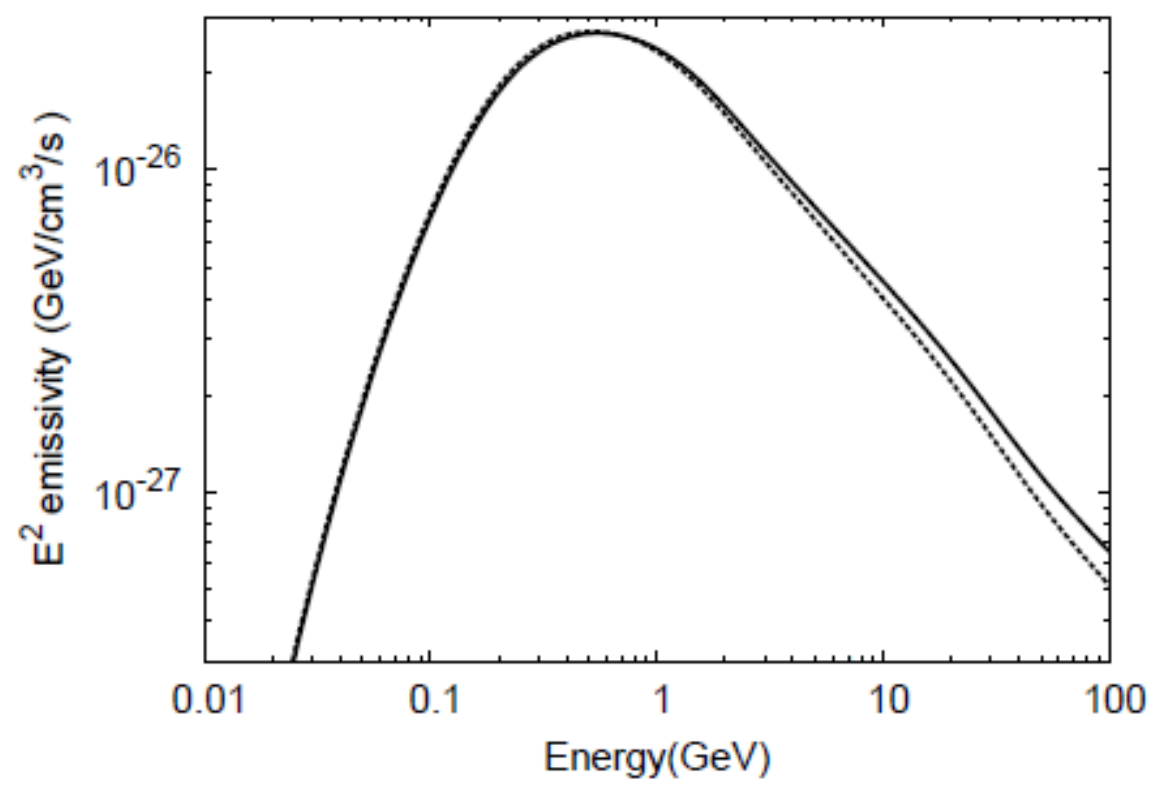

Figure 3.2: The $\gamma$-ray SED from $\pi^{0}$ decay. The normalization is arbitrary and $\alpha=2.85$. Image modified from Yang et al. (2018).

determined, all the parameters in the RIAF are determined, making the computation of the $\gamma$-ray spectrum due to the cooling process of ions almost unique (Oka and Manmoto, 2003). The $\gamma$-ray spectrum produced from $\pi^{0}$ decay is given by (Stecker, 1971)

$$
f_{\gamma}\left(E_{\gamma}\right)=2 \int_{E_{\min }}^{\infty} \frac{f_{\pi}\left(E_{\pi}\right)}{\sqrt{E_{\pi}^{2}-m_{\pi}^{2}}} d E_{\pi} \quad \text { photons } \mathrm{cm}^{-3} \mathrm{~s}^{-1} \mathrm{GeV}^{-1}
$$

where the factor of 2 is to account that each $\pi^{0}$ generates $2 \gamma$-rays, $E_{\pi}$ is the $\pi^{0}$ energy in $\mathrm{GeV}, m_{\pi}$ is the rest mass of the $\pi^{0}$ in $\mathrm{GeV} \mathrm{c} c^{-2}$, and $f_{\pi}\left(E_{\pi}\right)$ is the $\pi^{0}$ spectrum. The parameter $E_{\text {min }}$ is the minimum $\pi^{0}$ energy required to produce a $\gamma$-ray with energy $E_{\gamma}$, given by

$$
E_{\min }=E_{\gamma}+\frac{m_{\pi}^{2}}{4 E_{\gamma}}
$$

The SED from Equation 3.3 has a distinct bump around $1 \mathrm{GeV}$ (Figure 3.2), for which the shape and position depends on the spectral index $\alpha$ of the proton energy distribution in the RIAF (Yang et al., 2018). This bump was observed by Fermi-LAT in several supernova remnants (Ackermann et al., 2013), like, e.g., IC 443 and W44, but was never observed in AGNs.

Unfortunately, the proton energy distribution in a RIAF is not well known. For a hard enough distribution $(\alpha<2)$, the bump seen in Figure 3.2 can even disappear (Yang et al., 2018). This problem rises due to our lack of knowledge on the mechanism guiding 
particle acceleration in RIAFs, which make us unable to tell whether the process leads to a relativistic Maxwell-Boltzmann (i.e., thermal) or power-law (i.e., non-thermal) distribution (Mahadevan et al., 1997).

\subsection{Inverse Compton scattering}

In the collimated jets of AGNs, the main process producing $\gamma$-rays is the inverse Compton scattering. Through this process, soft photons with typical energies in the radio-tooptical range are boosted to the $\gamma$-ray regime by relativistic electrons. The equations shown below characterize the main properties of this phenomenon (Rybicki and Lightman, 2008).

Before we derive the equations describing the inverse Compton scattering, lets consider the non-relativistic Thomson scattering in the rest frame of an electron. In this case, the Poynting flux (power per unit area) of a plane wave incident on the electron is

$$
\vec{S}=\frac{c}{4 \pi} \vec{E} \times \vec{H}=\frac{c}{4 \pi}|\vec{E}|^{2}=c U_{r a d}
$$

where $U_{\text {rad }}$ is the energy density of the incident radiation. The electric field of the incident radiation then accelerates the electron, which in turn emit radiation according to Larmor's equation. The emitted power is simply the scatter of a portion of the incoming radiation with no net transfer of energy between the radiation and the electron:

$$
P=\sigma_{T} c U_{\text {rad }}
$$

where $\sigma_{T}$ is the Thomson cross-section. In other words, the electron will extract from the incident radiation the amount of power crossing the area $\sigma_{T}$ and reradiate that power back to the environment. However, if the electron originally has a relativistic speed in the rest frame of the observer, the observed power needs to be corrected to

$$
P=\sigma_{T} c U_{r a d}^{\prime}
$$

In order to transform $U_{\text {rad }}^{\prime}$ into $U_{\text {rad }}$, lets suppose that the electron is moving with a speed $v=v_{x}$ in the rest frame of the observer. The time between the arrival of two photons 
in the observer's frame is

$$
\Delta t=\Delta t^{\prime}[\gamma(1+\beta \cos \theta)]
$$

where $\beta \equiv v / c, \theta$ is the photon incidence angle relative to the $\mathrm{x}$ axis and $\gamma$ is the electron's Lorentz factor. From Equation 3.5, we can easily derive the relativistic Doppler effect if we interpret the time interval $\Delta t$ as the time between the arrivals of two successive cycles of an electromagnetic wave with frequency $\nu=\Delta t^{-1}$ in the observer's frame and $\nu^{\prime}=\Delta t^{\prime-1}$ in the electron's frame. Then

$$
\nu^{-1}=\nu^{\prime-1}[\gamma(1+\beta \cos \theta)] \quad \rightarrow \quad \nu^{\prime}=\nu[\gamma(1+\beta \cos \theta)]
$$

This means that in the electron's frame, the incoming photon energy $h \nu^{\prime}$ is multiplied by $\gamma(1+\beta \cos \theta)$. Similarly, the photon number density in the electron's frame, $N^{\prime}$, depends on the same factor $N^{\prime}=N[\gamma(1+\beta \cos \theta)]$. By considering a monochromatic background radiation field, we have $U_{\text {rad }}=N h \nu$ in the observer's frame. Equivalently, in the electron's frame we have

$$
U_{\text {rad }}^{\prime}=N^{\prime} h \nu^{\prime}=N[\gamma(1+\beta \cos \theta)] h \nu[\gamma(1+\beta \cos \theta)]=U_{\text {rad }}[\gamma(1+\beta \cos \theta)]^{2}
$$

The total energy density in the electron's frame for an isotropic and monochromatic radiation field in the observer's frame is obtained by integrating over all directions:

$$
U_{\text {rad }}^{\prime}=\frac{U_{\text {rad }}}{4 \pi} \int_{\phi=0}^{2 \pi} \int_{\theta=0}^{\pi}[\gamma(1+\beta \cos \theta)]^{2} \sin \theta d \theta d \phi=\frac{U_{\text {rad }} \gamma^{2}}{2} \int_{\theta=0}^{\pi}(1+\beta \cos \theta)^{2} \sin \theta d \theta
$$

where $\phi$ is the azimuthal angle around the $\mathrm{x}$ axis. This integral yields

$$
U_{\text {rad }}^{\prime}=\frac{4 U_{\text {rad }}\left(\gamma^{2}-1 / 4\right)}{3}
$$

which when substituted in Equation 3.4 gives

$$
P=\frac{4 \sigma_{T} c U_{r a d}\left(\gamma^{2}-1 / 4\right)}{3}
$$

As the initial power before the scattering was $P=\sigma_{T} c U_{\text {rad }}$, the net gain in power due to 
inverse Compton scattering is

$$
P_{I C}=\frac{4 \sigma_{T} c U_{r a d}\left(\gamma^{2}-1 / 4\right)}{3}-\sigma_{T} c U_{r a d}
$$

or

$$
P_{I C}=\frac{4 \sigma_{T} c \beta^{2} \gamma^{2} U_{r a d}}{3}
$$

where we used $\left(\gamma^{2}-1\right)=\beta^{2} \gamma^{2}$. If compared with the corresponding synchrotron power

$$
P_{\text {syn }}=\frac{4 \sigma_{T} c \beta^{2} \gamma^{2} U_{B}}{3}
$$

we find a fairly simple ratio of inverse Compton to synchrotron radiation losses:

$$
\frac{P_{I C}}{P_{\text {syn }}}=\frac{U_{\text {rad }}}{U_{B}}
$$

Equation 3.7 holds as long as the background photons have $\gamma h \nu \ll m_{e} c^{2}$ (i.e., Thomson scattering in the rest frame) and states that in a medium with a high density of photons, like the surroundings of an AGN, the inverse Compton power becomes an important process for cooling the relativistic electrons.

The higher the energy of the electrons, the higher is the frequency of the scattered photons. Lets suppose again an isotropic monochromatic field in the observer's frame where the photons have frequency $\nu_{0}$. If the photons are scattered by a single ultrarelativistic electron moving along the $\mathrm{x}$ axis, relativistic aberration causes most of the photons to approach nearly head on in the electron's rest frame. The relativistic Doppler effect shown in Equation 3.6 thus gives the frequency $\nu_{0}^{\prime}$ in the electron's rest frame for a photon approaching near the $\mathrm{x}$ axis $(\theta \sim 0)$ :

$$
\nu_{0}^{\prime} \approx \nu_{0}[\gamma(1+\beta)]
$$

In the electron's rest frame, Thomson scattering produces radiation with the same frequency as the incident radiation; the scattered photons thus have $\nu^{\prime}=\nu_{0}^{\prime}$. In the observer's frame, however, relativistic aberration beams the scattered photons in the x direction and the frequency $\nu$ of the scattered photons is given, again, by the relativistic Doppler 
formula:

$$
\nu \approx \nu^{\prime}[\gamma(1+\beta)] \approx \nu_{0}[\gamma(1+\beta)]^{2}
$$

which, in the ultrarelativistic limit $\beta \approx 1$, becomes

$$
\nu \approx 4 \gamma^{2} \nu_{0}
$$

This is the maximum frequency of the upscattered photons in the observer's frame. Oblique collisions $(\theta>0)$ result in lower frequencies.

\subsubsection{Synchrotron Self-Compton and external Compton}

The inverse Compton scattering of synchrotron radiation by the same relativistic electrons that produced the synchrotron emission is termed synchrotron self-Compton. Equation 3.8, describes the synchrotron power emitted by a single electron. If we multiply this equation by a number $N$ of electrons, then $U_{\text {rad }}$ is also multiplied by $N$ (i.e., $U_{\text {rad }} \propto N$ ). The inverse Compton power (Equation 3.7) from the scattering of synchrotron photons then scales with $P_{I C} \propto N \times U_{\text {rad }} \propto N^{2}$. Hence, for any synchrotron source, it is expected the presence of inverse Compton radiation which strongly depends on the electrons density distribution.

The relativistic electrons in an AGN jet can also scatter thermal photons from the accretion disk or even from the cosmic microwave background in a phenomenon known as external Compton scattering. The shape of the emitted spectrum, however, will always resemble that of synchrotron radiation (a power law with a cut off), as it depends on the non-thermal energy distribution of electrons instead of the original spectrum of the background radiation.

\section{$3.4 \gamma-\gamma$ absorption}

The interaction of $\gamma$-rays with lower energy target photons can produce $e^{+}+e^{-}$pairs once the following kinematic threshold condition is reached

$$
(h \nu)\left(h \nu_{t}\right)>\frac{2\left(m_{e} c^{2}\right)^{2}}{1-\cos \alpha}
$$


where $\alpha$ is the angle between the incident directions of the photons, $h \nu$ is the energy of the $\gamma$-ray and $h \nu_{t}$ is the energy of the target photon. This effect leads to a suppression of the $\gamma$-ray flux from a source if the medium in which the $\gamma$-rays are propagating has a high density of soft photons or if the $\gamma$-rays travel a long enough distance among a low density background of soft photons.

The $e^{+}+e^{-}$pairs produced in these interactions tend to be highly relativistic and move in the same direction as the original $\gamma$-ray in order to conserve linear momentum. For a given $\gamma$-ray dimensionless energy $\epsilon=h \nu /\left(m_{e} c^{2}\right)$, the polarization averaged cross-section for pair production $\sigma_{\gamma \gamma}$ depends only on the target photon dimensionless energy $\epsilon_{t}$ and incidence angle $\alpha$ (see eq. 13-40 in Jauch and Rohrlich, 2012):

$$
\sigma_{\gamma \gamma}\left(\epsilon_{t}, \alpha \mid \epsilon\right)=\frac{3 \sigma_{T}}{16}\left(1-s^{2}\right)\left[\left(3-s^{4}\right) \ln \left(\frac{1+s}{1-s}\right)-2 s\left(2-s^{2}\right)\right]
$$

where $s \equiv \sqrt{1-2 /\left(\epsilon \epsilon_{t}[1-\cos \alpha]\right)}$, and $\sigma_{T}$ is the Thomson cross-section (see also Böttcher and Els, 2016, for more details). The $\sigma_{\gamma \gamma}$ cross-section then rises steeply from the threshold $\operatorname{energy}(s=0)$ up to a maximum $\approx \sigma_{T} / 4$ at $s \approx 0.7$ and decreases approximately $\propto 1 / \epsilon_{t}$ towards higher energies, as follows

$$
\sigma_{\gamma \gamma}\left(\epsilon_{t}, \alpha \mid \epsilon\right)=\frac{3 \sigma_{T}}{8} \frac{1}{\epsilon \epsilon_{t}(1-\cos \alpha)}\left[\ln \left(2 \epsilon \epsilon_{t}[1-\cos \alpha]\right)-2\right]
$$

By considering the maximum cross-section $(s \approx 0.7)$, we have

$$
s \approx 0.7 \approx \sqrt{1-\frac{1}{\epsilon \epsilon_{t}}} \rightarrow \epsilon_{t} \approx \frac{2}{\epsilon} \rightarrow \nu_{t} \approx \frac{2}{\nu} \frac{\left(m_{e} c^{2}\right)^{2}}{h^{2}}
$$

where we have considered a head on interaction $(\cos \alpha=-1)$. Hence, for a $1 \mathrm{TeV} \gamma$-ray $\left(\nu=2.418 \times 10^{26} \mathrm{~Hz}\right)$, the optimum target for a head on interaction is an infrared photon with $\nu_{t} \approx 1.2 \times 10^{14} \mathrm{~Hz}$. In the case of a neutron star, where the thermal black body radiation peaks in X-rays $\left(\nu_{t}=6.2 \times 10^{16} \mathrm{~Hz}\right.$, for a surface temperature of $T_{N S} \approx 600000$ $\mathrm{K}$ ), Equation 3.10 implies that the X-rays will better interact with $\gamma$-rays of a few $\mathrm{GeV}$ (i.e., $\sim 4 \mathrm{GeV}$ ).

The total optical depth, $\tau$, characterizes the absorption of a $\gamma$-ray of dimensionless energy $\epsilon$ when crossing a target photon field with differential number density $n\left(\epsilon_{t}\right)$. For a 
homogeneous and isotropic target photon field, the optical depth is (Herterich, 1974)

$$
\tau(\epsilon)=R \int_{2 / \epsilon}^{\infty} n\left(\epsilon_{t}\right) \bar{\sigma}_{\gamma \gamma} d \epsilon_{t}
$$

where $R$ is the size of the field and $\bar{\sigma}_{\gamma \gamma}$ is the cross-section averaged over all incoming directions. The $\gamma$-ray intensity then decreases as $I_{\gamma}=I_{\gamma 0} e^{-\tau}$. As the optical depth for a $\gamma$-ray is determined by a relatively narrow band of target photons centered at $\mathrm{s}=0.7$, a useful order of magnitude estimate is given by

$$
\tau(\epsilon) \approx R\left(\sigma_{T} / 4\right) n_{t} \times \frac{4}{\epsilon}
$$

where we have approximated $\bar{\sigma}_{\gamma \gamma}$ by a rectangular function with height $\sigma_{T} / 4$ and width $2 \times E_{\min } \rightarrow 2 \times 2 / \epsilon$. Equation 3.11 indicates that $\gamma$-rays cannot propagate in luminous environments (large $n_{t}$ ). For a luminous AGN, for instance, $\mathrm{TeV} \gamma$-rays generated close to the black hole will quickly interact with infrared photons from the accretion disk and hence never escape from the AGN. A remaining possibility for observing high energy photons from near the event horizon is the expected $\mathrm{TeV}$ emission from black hole outer magnetospheric gaps in LLAGNs (Rieger, 2011). 
Chapter 4

\title{
The gamma-ray emission from globular clusters
}

\author{
This thesis chapter is a shortened version of the paper "Milky Way \\ globular clusters in $\gamma$-rays: analyzing the dynamical formation of millisecond \\ pulsars" by de Menezes et al. (2019), published in the journal Monthly Notices \\ of the Royal Astronomical Society, volume 486, number 1. The original work \\ can be found online here.
}

\section{Abstract}

Globular clusters (GCs) are evolved stellar systems containing entire populations of millisecond pulsars (MSPs), which are efficient $\gamma$-ray emitters. Observations of this emission can be used as a powerful tool to explore the dynamical processes leading to binary system formation in GCs. In this work, 9 years of Fermi Large Area Telescope data are used to investigate the $\gamma$-ray emission from all GCs in the Milky Way. 23 clusters are found as $\gamma$-ray bright, with 2 of them never having been reported before. We also found that magnetic braking probably has a smaller impact on the formation rate of binary systems in metal-rich GCs than previously suggested, while a large value for the two-body encounter rate seems to be a necessary condition. The influence of the encounter rate per formed binary is for the first time explored in conjunction with $\gamma$-ray data, giving evidence that if this quantity is very high, binary systems will get destroyed before having time to evolve into MSPs, thus decreasing the total number of MSPs in a GC. We found no extended emission even for clusters whose optical extent is $\approx 0.5^{\circ}$; all of them are point-like sources spatially in agreement with the optical cores of the GCs, supporting previous X-rays results of heavier objects sinking into the clusters' cores via dynamical friction. 


\subsection{Introduction}

Since the first detection of $\gamma$-rays from 47 Tucanæ (Abdo et al., 2009) with the Fermi Large Area Telescope (LAT), globular clusters (GCs) have become a new class of $\gamma$-ray source. Previous observations in the 90's with the Energetic Gamma-ray Experiment (EGRET) on board the Compton Gamma-ray observatory found no signal of $\gamma$-ray emission from these sources but resulted in important flux upper limits for more than a dozen of them (Michelson et al., 1994). By studying GCs in $\gamma$-rays, we can learn about the dynamical evolution of these systems, as well as the mechanisms behind the formation of their millisecond pulsar (MSP) populations.

The $\gamma$-ray emission from GCs is attributed to their large number of MSPs (Bednarek and Sitarek, 2007; Abdo et al., 2010; Freire, 2012; Caraveo, 2014), which are known to be efficient $\gamma$-ray emitters (Chen, 1991; Harding et al., 2005). The recent detection of pulsed $\gamma$-ray emission from some GCs has further strengthened this connection (Freire et al., 2011; Johnson et al., 2013). The populations of MSPs in these systems ${ }^{1}$, as the descendants of low-mass X-ray binaries (LMXBs), are believed to be formed as a natural consequence of frequent stellar encounters (Pooley et al., 2003), with the high number density of stars within a GC providing an excellent laboratory to test scenarios for compact binary formation. Evidence favoring this dynamical origin, i.e., positive correlations between the cluster's $\gamma$-luminosity $\left(L_{\gamma}\right)$ with its stellar encounter-rate $(\Gamma)$ and with its metallicity $([\mathrm{Fe} / \mathrm{H}])$ have been found in the past few years (Abdo et al., 2010; Hui et al., 2010; Bahramian et al., 2013; Hooper and Linden, 2016; Lloyd et al., 2018). In interpretations of the former, the stars are assumed to be captured one by the other at a rate proportional to $\Gamma \propto \rho_{0}^{3 / 2} r_{c}^{2}$, where $\rho_{0}$ is the central luminosity density and $r_{c}$ is the cluster core radius (Verbunt, 2003a). In the latter, for a metal-rich cluster, magnetic braking can be more efficient, facilitating orbital decay in binary systems as well as a higher probability of MSP formation (Hui et al., 2010; Tam et al., 2016).

In contrast with other pulsars, MSPs begin as spun-down neutron stars in binary systems where the companion star is massive enough to evolve into a giant and overflow the Roche limit of the system (Lorimer, 2001). The neutron star is then spun-up and wakes up as a recycled pulsar by accreting matter and increasing angular momentum at

\footnotetext{
${ }^{1}$ List containing all known pulsars in GCs: https://www.naic.edu/ pfreire/GCpsr.html
} 
the expense of the orbital angular momentum of the binary system (Alpar et al., 1982). Due to their weak surface magnetic fields, MSPs lose their larger store of rotational kinetic energy much more slowly than common pulsars (Lorimer et al., 2005), remaining luminous for up to billions of years. In MSPs, $\gamma$-rays are mainly produced in magnetosphereric emission sites, likely driven by inverse Compton scattering, synchrotron and curvature radiation (Sturrock, 1971; Harding et al., 1978; Arons, 1983; Cheng et al., 1986; Bednarek and Sitarek, 2007).

In this work we analyze all Milky Way globular clusters listed in Harris (1996), using 9 years of Fermi-LAT data as an effort for detecting them and characterizing their $\gamma$-ray emission and MSP formation scenarios. The observations and cuts on Fermi-LAT data are described in Section 4.2; followed by the light curve analysis and study of correlations between $\gamma$-ray emission, encounter rate and metallicity, in Section 4.3. Sections 4.4 and 4.5 discuss the influence of magnetic braking on the formation of MSPs and present our conclusions.

\subsection{Data selection and analysis}

The analyzed sample consists of all 157 Milky Way GCs as listed in Harris (1996 - 2010 edition). For 25 of them, $\gamma$-ray emission was previously described in Acero et al. (2015) and Hooper and Linden (2016), although we do not confirm four of these detections (2MASSGC02, M15, NGC 6342 and Pal6). Each GC treated here was observed with Fermi-LAT during a 9-year period ranging from August 5th 2008 to August 5th 2017 (mission elapsed time 239587201-523584005). We analyzed the data using the Fermi Science Tools v10r0p5, fermipy python package v0.16.0 (Wood et al., 2017) and Pass 8 (Atwood et al., 2013), which present better energy and angular resolution as well as an increased effective area and energy range than its predecessor Pass 7.

Following standard procedures ${ }^{2}$, we selected the data for each source within a $12^{\circ} \times 12^{\circ}$ region-of-interest (ROI), centered on the GCs positions given in the GLOBCLUST catalog (Harris 1996 - 2010 edition), with energies ranging between $100 \mathrm{MeV}$ and $100 \mathrm{GeV}$ divided into 12 logarithmically spaced energy bins. We neglected photons with energies $>100$ $\mathrm{GeV}$, as the spectra of MSPs frequently presents an exponential cutoff behavior above only

\footnotetext{
${ }^{2}$ Fermi science tools and fermipy tutorials: https://fermi.gsfc.nasa.gov/ssc/data/analysis/ scitools/ and http://fermipy.readthedocs.io/en/latest/quickstart.html
} 
a few GeV (Abdo et al., 2010). Sources included in the Fermi-LAT Third Source Catalog (3FGL - Acero et al., 2015) and lying up to $5^{\circ}$ outside the ROIs were taken into account as well as all sources found with the fermipy function find_sources(sqrt_ts_threshold=5.0, min_separation=0.4). Only events belonging to the class source have been used (evclass $=128$ and evtype=3), and the filters applied with gtmktime were DATA_QUAL $>0$ and the recommended instrument configuration for science LAT_CONFIG $==1$. We applied a zenith angle cut of $90^{\circ}$ to avoid contamination from the Earth limb. For modeling the Galaxy and the extragalactic background emission, we adopted the Galaxy background model gll_iem_v06.fits and the isotropic spectral template iso_P8R2_SOURCE_V6_v06.txt.

All sources have been investigated by means of binned likelihood analysis (gtlike tool - MINUIT algorithm). To quantify the significance among the detections, we computed a test statistic (TS), defined as $T S=2\left(\mathcal{L}_{1}-\mathcal{L}_{0}\right)$, where the term inside parentheses is the difference between the maximum log-likelihoods with $\left(\mathcal{L}_{1}\right)$ and without $\left(\mathcal{L}_{0}\right)$ modeling the source. The chosen criteria for detection was $T S>25$, corresponding formally to a significance slightly above $4 \sigma$ (Mattox et al., 1996). If the detected GC belonged to 3FGL, we modelled its spectrum according to its description there. If a detected GC was not listed in 3FGL (post-3FGL clusters, from now on), we modelled its spectrum with a power-law. For all sources lying within a radius of $5^{\circ}$ from the center of the ROIs, we left the normalization parameter free to vary.

We added a point sources consistent with the optical center of the GCs to the models for each GC in the analysed sample and a TS residuals map was constructed for each one of them. These residuals maps were used together with aperture photometry light curves to obtain a clean sample of GCs, where only sources with a clear indication for an isolated $\gamma$-ray point-like emission spatially coincident with the optical position of the cluster and presenting steady light curves were taken into account. All detections are described in Section 4.3, while the flux upper limits for all non-detected GCs are shown in Appendix 4.6 . 


\subsection{Results}

\subsubsection{Detections}

Among the 157 GCs, 23 presented $\gamma$-ray emission spatially coincident with the optical center of the clusters, as shown in Table 4.1, which is segmented in three panels: the upper one shows the 15 GCs cataloged in 3FGL; the middle panel shows the post-3FGL clusters, and the last/bottom panel shows the new GCs candidates detected in this work.

\begin{tabular}{l|c|c|c|c} 
Cluster & SM & $\begin{array}{c}\text { Luminosity } \\
10^{34} \mathrm{erg} \mathrm{s}^{-1}\end{array}$ & $\begin{array}{c}\text { Energy flux } \\
10^{-12} \mathrm{erg} \mathrm{cm}^{-2} \mathrm{~s}^{-1}\end{array}$ & TS \\
\hline 47 Tuc & LP & $6.26 \pm 0.19$ & $25.81 \pm 0.77$ & 5604 \\
Terzan5 & LP & $42.39 \pm 1.54$ & $74.41 \pm 2.71$ & 3854 \\
M62 & LP & $8.96 \pm 0.55$ & $16.2 \pm 0.99$ & 1036 \\
NGC6388 & LP & $29.34 \pm 1.24$ & $25.01 \pm 1.06$ & 880 \\
R Cent & LP & $3.57 \pm 0.26$ & $11.03 \pm 0.8$ & 863 \\
2MS-GC01 & LP & $8.89 \pm 0.54$ & $57.33 \pm 3.46$ & 731 \\
NGC6440 & PL & $25.64 \pm 1.4$ & $29.66 \pm 1.62$ & 518 \\
NGC6316 & PL & $22.38 \pm 1.62$ & $17.29 \pm 1.25$ & 276 \\
NGC6441 & PL & $27.37 \pm 1.86$ & $17.0 \pm 1.16$ & 331 \\
NGC6752 & PL & $1.02 \pm 0.1$ & $5.32 \pm 0.54$ & 149 \\
NGC6652 & LP & $4.19 \pm 0.58$ & $3.5 \pm 0.48$ & 129 \\
M80 & PL & $7.10 \pm 0.95$ & $5.94 \pm 0.79$ & 92 \\
NGC2808 & PL & $4.89 \pm 0.67$ & $4.43 \pm 0.61$ & 81 \\
NGC6541 & PL & $3.34 \pm 0.44$ & $4.97 \pm 0.66$ & 78 \\
NGC6717 & PL & $1.76 \pm 0.38$ & $2.92 \pm 0.64$ & 31 \\
\hline Glimp01 & PL & $9.83 \pm 1.29$ & $46.54 \pm 6.09$ & 286 \\
Glimp02 & PL & $8.6 \pm 1.07$ & $23.74 \pm 2.96$ & 98 \\
NGC6397 & PL & $0.35 \pm 0.05$ & $5.5 \pm 0.79$ & 64 \\
NGC6139 & PL & $8.22 \pm 1.31$ & $6.73 \pm 1.07$ & 59 \\
M12 & PL & $0.88 \pm 0.17$ & $3.20 \pm 0.61$ & 43 \\
M5 & PL & $1.52 \pm 0.33$ & $2.25 \pm 0.49$ & 31 \\
\hline M14 & PL & $3.17 \pm 2.49$ & $3.06 \pm 2.4$ & 29 \\
M79 & PL & $3.61 \pm 0.82$ & $1.81 \pm 0.41$ & 26 \\
\hline
\end{tabular}

Table 4.1 - Observations of 23 globular cluster candidates with Fermi-LAT in an energy range from $100 \mathrm{MeV}$ up to $100 \mathrm{GeV}$. The upper panel displays the 3FGL associations, the middle panel shows the post-3FGL associations and the bottom panel shows the new GC candidates. The adopted spectral models (SM) are indicated on the second column, where $\mathrm{PL}=$ power-law and LP $=\log$ Parabola. The computation of the luminosities was performed assuming isotropic $\gamma$-ray emission and using the distances available in the GLOBCLUST catalog. 

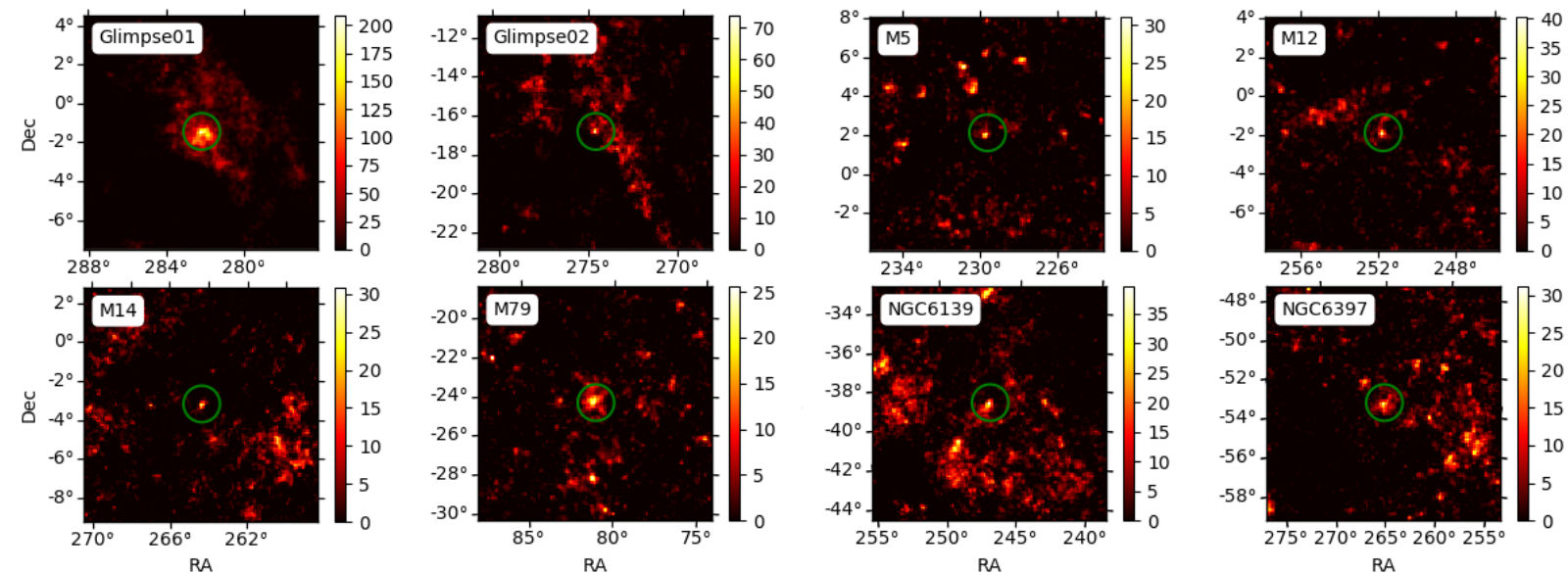

Figure 4.1: Mosaic of TS residuals maps for the firmly detected post-3FGL sources. The green circles guide the readers to the center of the maps.

\subsubsection{Fermi-LAT 8 years source list}

Cross checking the detections in this work with the ones listed on the preliminary LAT 8-year Point Source List (FL8Y3), some differences are found. Six sources (2MASS-GC02, M92, NGC 362, NGC 6304, NGC 6342 and Terzan 1) associated to GCs in FL8Y are not significant detections in this work; and only one of the GCs detected in this work is not listed in FL8Y (M79). All of these clusters (with exception of Terzan 1) are very close to the detection threshold. These differences may be related to the alternative likelihood method adopted in FL8Y (weighted likelihood ${ }^{4}$ ) and may vary depending on the analyzed region. The clusters 2MASS-GC02, NGC 362 and Terzan 1, for instance, are located on very complicated regions of the sky with bright diffuse emission. Also M92 and M79 are $\sim 1^{\circ}$ apart from very bright sources. Future analyses including the 4 th source catalog of the Fermi-LAT (4FGL, in preparation) will benefit from a new Galactic diffuse $\gamma$-ray emission model based on Pass 8 data, allowing for better results.

\subsubsection{Point-like sources}

The spatial consistency between the $\gamma$-ray and optical/infrared emission for the firmly detected post-3FGL sources can be checked in Figure 4.1, where the low-energy centers of the GCs are always coincident with the centers of the TS residuals maps. All maps have evidence for $\gamma$-ray emission with a maximum lying less than $0.15^{\circ}(\sim 2$ pixels $)$ from

\footnotetext{
3 https://fermi.gsfc.nasa.gov/ssc/data/access/lat/fl8y/

4 https://fermi.gsfc.nasa.gov/ssc/data/access/lat/fl8y/FL8Y_description_v8.pdf
} 


\begin{tabular}{l|c|c|c|c} 
Cluster & RA & Dec & $r_{1 \sigma}$ & $\alpha$ \\
\hline M14 & $264.412^{\circ}$ & $-3.238^{\circ}$ & $0.035^{\circ}$ & $0.014^{\circ}$ \\
M79 & $81.120^{\circ}$ & $-24.410^{\circ}$ & $0.140^{\circ}$ & $0.134^{\circ}$
\end{tabular}

Table 4.2 - Best fit position for M14 and M79. $r_{1 \sigma}$ is the $1 \sigma$ error circle radius and $\alpha$ is the angular separation between the position of the $\gamma$-ray detection and the optical center of the cluster. In both cases $\alpha$ lies inside the $1 \sigma$ uncertainty region. Positions are given in J2000 coordinates.

their centers. TS maps for 3FGL clusters are not shown here, as their emission is generally easily visible in their counts maps. The two new sources found in this work, M14 and M79, had their best fitted positions obtained with the function localize(), available in fermipy, which found a $\gamma$-ray-optical/infrared spatial separation within $1 \sigma$ uncertainty radius for both cases (Table 4.2).

\subsubsection{Extended source analysis}

For $\Omega$ Centauri, 47 Tucanæ and NGC 6397, the three $\gamma$-ray bright GCs with largest optical angular diameter $\left(\gtrsim 0.5^{\circ}\right)$, we have tested extended emission models. The extended emission templates were created in two ways: with DSS optical maps available in NASA's SkyView ${ }^{5}$ virtual telescope (McGlynn et al., 1998) and with 25 2D-Gaussian source templates with sizes ranging from $0.003^{\circ}$ to $1^{\circ}$. For all tests, the likelihood ratio method favored the point-like model instead of the extended emission models, which, in the most optimistic cases, presented TS values of only $T S_{\text {ext }}=0.73$ for $\Omega$ Centauri $\left(R_{68}=0.055 \pm 0.031\right), T S_{\text {ext }}=0.38$ for 47 Tucanæ $\left(R_{68}=0.033 \pm 0.022\right)$ and $T S_{\text {ext }}=0.00$ for NGC $6397\left(R_{68}=0.003 \pm 0.080\right)$. These results are in agreement with heavier objects sinking into the clusters' cores via dynamical friction (Fregeau et al., 2003), as both binaries and single MSPs are significantly more massive than typical stars in a GC. The same conclusion is reached from X-ray observations of 47 Tucanæ (Edmonds et al., 2003; Heinke et al., 2005), where the cluster's X-ray source population is highly concentrated in its core.

\subsubsection{Light curves}

The intrinsic variability of MSPs completely disappears when the observations span through timescales much longer than the MSP typical revolution time. A light curve with bins of months is thus expected to be quiescent (Abdo et al., 2010), where its variability is attributed only to statistical fluctuations. High levels of variability in a light curve with

\footnotetext{
${ }^{5}$ https://skyview.gsfc.nasa.gov/current/cgi/query.pl
} 

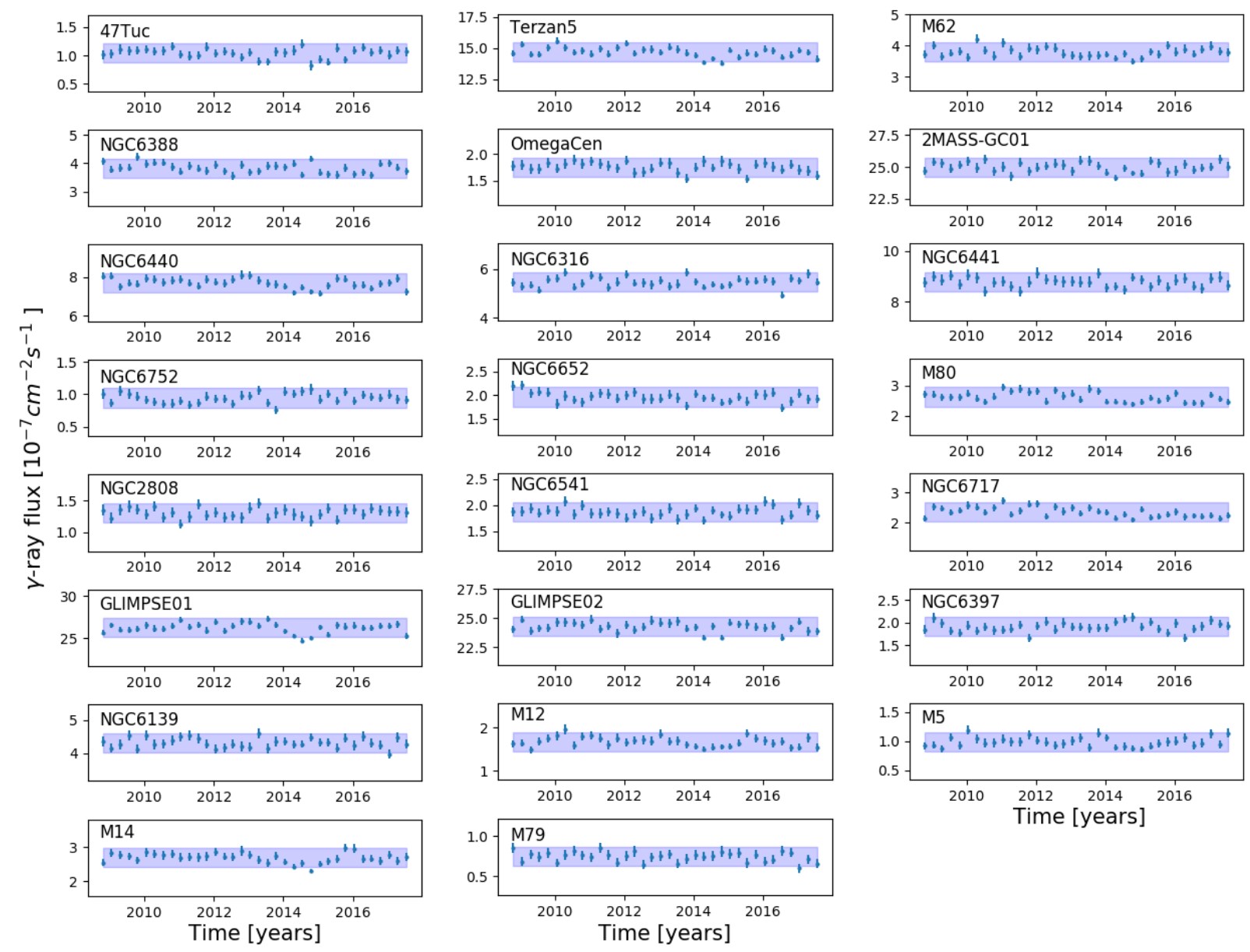

Figure 4.2: Aperture photometry light curves for the $23 \gamma$-ray bright GCs. Each time bin corresponds to 3 months of data in an energy range from $100 \mathrm{MeV}$ to $100 \mathrm{GeV}$ and aperture radius of $1^{\circ}$. All sources exhibit weak variability, showing no flares with peaks significantly above the $2 \sigma$ deviation level (blue band). None of the clusters have been excluded from the analyzed sample based on their light curves.

such timescales are unlikely to be associated with a GC; they could nevertheless be due to a background active galactic nucleus.

To test for variability, we have created aperture photometry light curves with gtbin for every single $\gamma$-ray GC. The results are shown in Figure 4.2, where the data is binned in 3 -month intervals, with an aperture radius of $1^{\circ}$ and photon index fixed in 2 . All light curves analyzed show a quiescent behavior, as expected for GCs, with all data points lying within the $2 \sigma$ standard deviation level (blue band).

\subsubsection{Spectral emission models}

The high energy $\gamma$-ray spectra for 19 of the 23 detected GCs are shown in Figure 4.6 in Appendix 4.6. All spectra are reasonably well fitted by a logparabola or a power-law model, in agreement with the GCs discussed in Abdo et al. (2010). As no significant 
deviation from such models is observed, the traditional interpretation that the observed $\gamma$ ray emission is coming from populations of MSPs is assumed throughout this work. Other models are discussed below.

One possible mechanism for producing $\gamma$-rays in GCs is inverse Compton scattering (ICS) by a relativistic population of electrons in the intracluster medium (Bednarek and Sitarek, 2007). The spectra derived from such model, however, predict a hardening of the spectrum around 1-10 GeV (Bednarek and Sitarek, 2007; Lloyd et al., 2018). In this work, no strong evidence for a spectral hardening is found in this band (Figure 4.6).

Although dark matter annihilation has been proposed as another possible source of $\gamma$-rays in GCs (Brown et al., 2018), the lack of strong dynamical evidence (Moore, 1996; Haşegan et al., 2005) suggests that this process alone cannot explain the observed $\gamma$-ray emission.

The light curves shown in Figure 4.2 reduces the possibility of associating the observed $\gamma$-ray emission with cataclysmic variables within the clusters, as these sources are transient in nature (Abdo et al., 2010) and no significant variability is observed in the analyzed light curves.

\subsubsection{Correlated quantities}

Parameters such as the two-body encounter rate $\Gamma$ and metallicity $[\mathrm{Fe} / \mathrm{H}]$ are expected to influence the formation rate - and so the total number - of MSPs in a GC (see Section 4.1). For estimating the total number of MSPs, $N_{\mathrm{MSP}}$, within a cluster, a simple calculation is performed (Abdo et al., 2010):

$$
N_{\mathrm{MSP}}=\frac{L_{\gamma}}{\langle\dot{E}\rangle\left\langle\eta_{\gamma}\right\rangle}
$$

where $L_{\gamma}$ is the cluster's isotropic $\gamma$-ray luminosity, $\langle\dot{E}\rangle$ is the average power emitted during the spin down of MSPs and $\left\langle\eta_{\gamma}\right\rangle$ is the average efficiency with which the spin down power is converted into $\gamma$-ray luminosity. The isotropic energy luminosity is given by $L_{\gamma}=4 \pi r^{2} \epsilon_{\gamma}$, where $\epsilon_{\gamma}$ is the measured energy flux (Table 4.1) and $r$ is the distance to the cluster taken from Harris (1996 - 2010 edition). We adopted an average spin-down power and average spin-down-to- $\gamma$-ray efficiency of $\langle\dot{E}\rangle=(1.8 \pm 0.7) \times 10^{34} \mathrm{erg} \mathrm{s}^{-1}$ and $\left\langle\eta_{\gamma}\right\rangle=0.08$, respectively, for all clusters (Abdo et al., 2010). These values have been 
estimated from comparisons of the $\log \dot{P}$ distributions of Galactic field MSPs with the accelerated corrected $\log \dot{P}$ distribution for MSPs in 47 Tucanæ and by the average $\eta_{\gamma}$ efficiency of the nearest MSPs to date as described in Abdo et al. (2009). Equation (4.1) is a rough estimate of $N_{\gamma}$, as in some cases $L_{\gamma}$ may be dominated by the emission of a single MSP (Freire et al., 2011). Nevertheless, it should be good enough to at least establish an upper limit for the actual number of MSPs in a GC.
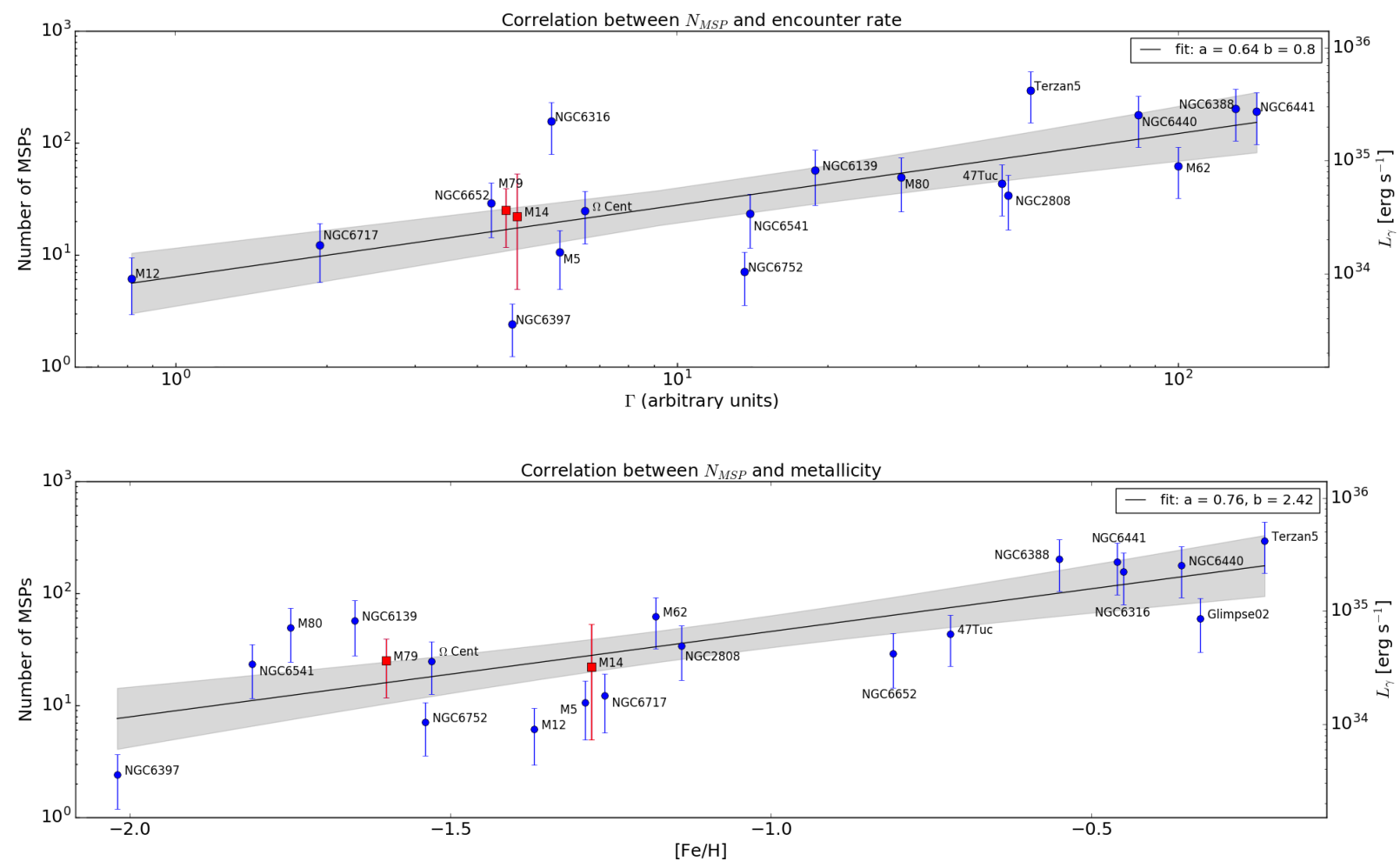

Figure 4.3: Correlation plots for $N_{\mathrm{MSP}} \times \Gamma$ and $N_{\mathrm{MSP}} \times[\mathrm{Fe} / \mathrm{H}]$ for the $\gamma$-ray bright GCs described in this work. The two new candidates for GCs found in this work are plotted as red squares. Sources lacking values of metallicity, central luminosity density or cluster's core radius in GLOBCLUST catalog are neglected. The shaded areas are the $1 \sigma$ confidence bands. For a version of the upper panel plot including upper limits, see Appendix 4.6.

The scatter plots for $N_{\mathrm{MSP}}$ versus $\Gamma$ and $N_{\mathrm{MSP}}$ versus $[\mathrm{Fe} / \mathrm{H}]$ are shown in Figure 4.3. For these plots, all $\gamma$-ray bright GCs with data values for metallicity and central luminosity density (needed for calculating $\Gamma$ ) available in the GLOBCLUST catalog are used. Note that the encounter rate is in arbitrary units and normalized such that $\Gamma=100$ for $\mathrm{M} 62$, as done in Abdo et al. (2010).

A simple linear least-squares regression was performed to the data displayed in Fig. 4.3. In the upper panel,

$$
\log N_{\mathrm{MSP}}=a \log \Gamma+b
$$




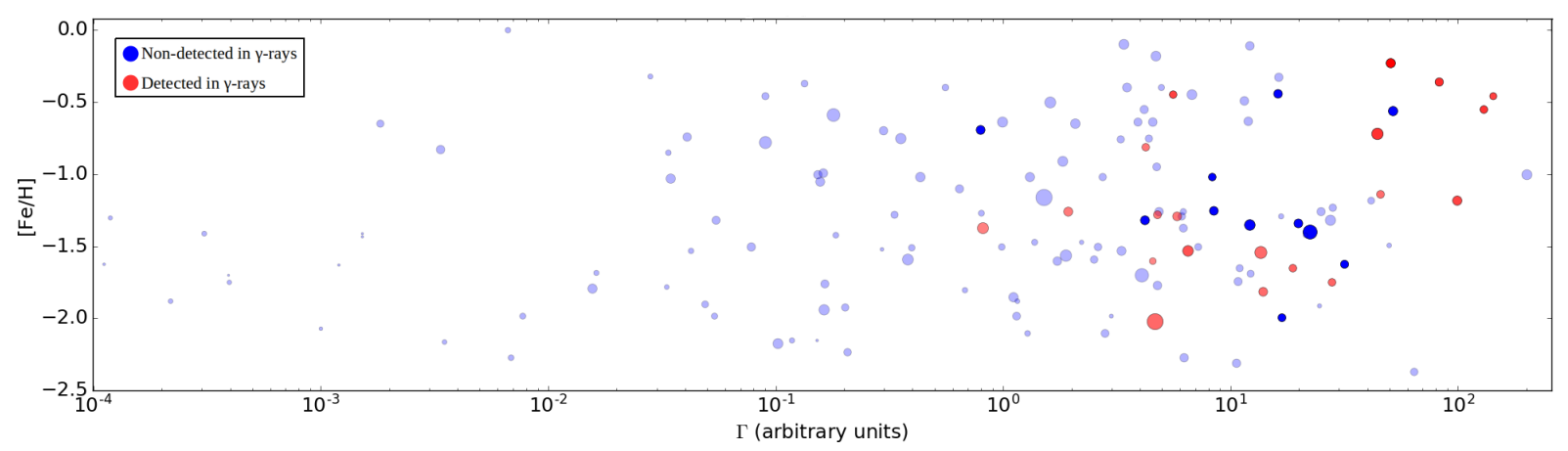

Figure 4.4: Influence of $[\mathrm{Fe} / \mathrm{H}]$ and $\Gamma$ for $143 \mathrm{GCs}$ in the Milky Way (those for which metallicity and encounter rate can be taken or calculated from data available in GLOBCLUST). The size of each point scales with distance: the closer the cluster, the larger the point. Blue points: GCs non-detected by Fermi-LAT. Clusters with $\Lambda>350$ are represented in dark blue. These sources are expected to be faint in $\gamma$-rays (see section 4.3.7). Red points: GCs detected in $\gamma$-rays. In this case, the darker the point, the larger the $\gamma$-ray energy flux. Detections are concentrated towards high values of encounter rate.

where $a=0.64 \pm 0.15, b=0.80 \pm 0.20$ with a mean deviation of $\Delta\left(\log N_{\mathrm{MSP}}\right)=0.40 \mathrm{dex}$ and a Pearson correlation coefficient $P_{\text {corr }}$ and $p$-value for testing non-correlation $p_{\mathrm{n}-\mathrm{c}}$ of $P_{\text {corr }}=0.72$ and $p_{\mathrm{n}-\mathrm{c}}=0.00034$, respectively. In the bottom panel,

$$
\log N_{\mathrm{MSP}}=a[\mathrm{Fe} / \mathrm{H}]+b
$$

with $a=0.76 \pm 0.14, b=2.42 \pm 0.17, \Delta\left(\log N_{\mathrm{MSP}}\right)=0.37$ dex, $P_{\text {corr }}=0.76$ and $p_{\mathrm{n}-\mathrm{c}}=$ $6.2 \times 10^{-5}$. Both correlations indicate that the $\gamma$-ray luminosity (or $N_{\mathrm{MSP}}$, since $N_{\mathrm{MSP}} \propto L_{\gamma}$ ) of a cluster increases with its $\Gamma$ and/or metallicity.

To test if these correlations are valid for all GCs in the Milky Way, Figure 4.4 shows the $[\mathrm{Fe} / \mathrm{H}] \times \Gamma$ scatter plot. As can be seen, all detected $\gamma$-ray clusters have a relatively large encounter rate, while their metallicities assume very dispersed values. This result may indicate that the magnetic braking effect is not significantly enhanced in metal-rich clusters, having a smaller role in the compact binary system formation rate than previously suggested (Hui et al., 2010; Tam et al., 2016). Although the metallicity and the $N_{M S P}$ (or $L_{\gamma}$ ) indeed present a correlation, as shown in Figure 4.3, the high metallicity may not be interpreted as causing the formation of MSPs (via magnetic braking). If the metallicity increased the MSP formation, then the detections (red dots) in Figure 4.4 should be concentrated in the upper part of the plot. On the other hand, the presence of MSPs is an indicative of a past full of supernovae explosions, which could enhance the clusters' environments with metals (see section 4.4 for a discussion). 


\subsubsection{Secondary encounters and binary disruption}

Besides the observed correlation between $N_{\mathrm{MSP}}$ and $\Gamma$, there are some aspects of the formation and evolution of MSPs not described by $\Gamma$, like the effects caused by secondary encounters. Once the binary is formed, it may undergo subsequent encounters, which may disrupt the system or even exchange binary members.

Analogously to the estimation of $\Gamma$ described in Section 4.1, one can estimate the encounter rate per formed binary as $\Lambda \propto \sqrt{\rho_{0}} / r_{c}$ (Verbunt, 2003b; Verbunt and Freire, 2014), where $\rho_{0}$ and $r_{c}$ are the central luminosity density and core radius respectively. In GCs with large values for $\Lambda$, the lifetime of binaries should be relatively short $(\tau=1 / \Lambda)$ before being disrupted or undergoing an exchange. The evolution of LMXBs in such clusters may be interrupted before their neutron stars become completely recycled, which may affect their overall population of MSPs. This behavior is perhaps what is seen in Figure 4.5, where a drop in $N_{\mathrm{MSP}}$ is evident for very large values of $\Lambda$ (normalized such that $\Lambda=100$ for M62). The high $\Lambda$ values for NGC 6752 and NGC 6397 can also explain why these clusters have such low $\gamma$-ray luminosity and appear as outliers in Figure 4.3.

Interestingly, GCs with intermediate values of $\Lambda$ are those with the largest populations of MSPs (Figure 4.5). This suggests that MSPs are preferentially formed in secondary exchange encounters, as only clusters with intermediate or high $\Lambda$ are likely to host pulsar binaries formed in this way (Verbunt and Freire, 2014).

High values for $\Lambda$ may also be the explanation for many of the non-detections shown in Figure 4.4. Many of the clusters with high $\Gamma$, which are roughly expected to be $\gamma$-ray bright, also have high $\Lambda$, which may negatively affect their MSP population. Non-detected clusters with $\Lambda>350$ are plotted in dark blue in Figure 4.4.

\subsection{Discussion}

In a scenario where the magnetic braking effect is significantly enhanced by a metalrich environment, high $\gamma$-ray fluxes should be seen for clusters with high metallicities; but this is not observed in Figure 4.4, which shows that metal-rich clusters are not necessarily efficient $\gamma$-ray emitters. This result suggests that a higher metallicity does not imply a significantly larger magnetic braking effect (and thus a higher MSP formation rate). Hypotheses for explaining the high metallicities in clusters crowded with MSP may be 


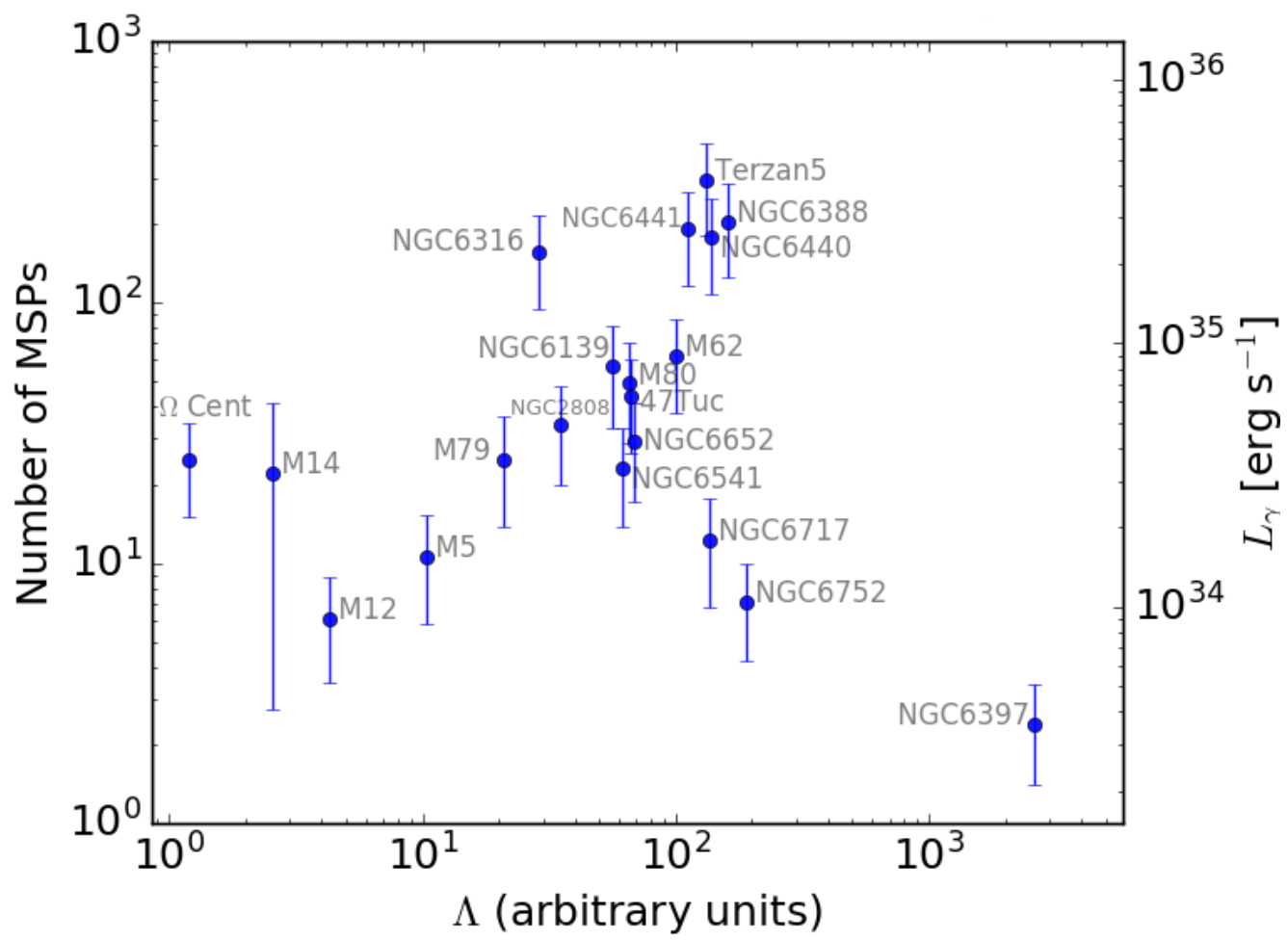

Figure 4.5: Impact of $\Lambda$ on the populations of MSPs. The largest populations of MSPs are concentrated in clusters with intermediate values of $\Lambda$, indicating that MSPs are preferentially formed in secondary exchange encounters.

related to the feedback of MSPs or their progenitors within the clusters environment.

At least 5 of the 23 clusters described in this work ( $\Omega$ Cen, NGC 2808, NGC 6397, NGC 6752 and M5) are abundant in Calcium (Lee et al., 2009). Calcium and other heavy elements can only be supplied to these systems via supernovae explosions (Timmes et al., 1995). As the gravitational potential well in present day clusters cannot retain most of the ejecta from such explosions (Baumgardt et al., 2008), it has been suggested by Lee et al. (2009) that these GCs are most likely relics of what were once the nuclei of primordial dwarf galaxies accreted and disrupted by the Milky Way (Bica et al., 2006). This supernova enrichment hypothesis is also supported by some GCs formation models, where stellar winds and supernova ejecta within proto-GCs are decelerated to speeds below the clusters' escape velocity by the pressure of the surrounding hot gas in which they are embedded (Brown et al., 1991). The metallicity of a cluster, in this context, is simply a function of its total number of supernovae (and not necessarily $N_{\text {MSP }}$ ). 


\subsection{Conclusions}

Nine years of Fermi-LAT data have been analyzed, revealing GC candidates characterized by quiescent $\gamma$-ray emission spatially coincident with the optical centers of the clusters. The novelty of this work is mainly:

- Evidence that metallicity does not have a significant impact on the MSPs formation. If a metal-rich environment is one of the causes of MSP formation (enhancing the magnetic braking), a concentration of detections (red dots) should be seen in the upper part of Figure 4.4. The results indicate that the MSP formation may be dominated by the encounter rate and encounter rate per binary rather than by an enhanced magnetic braking effect.

- It is the first time that the encounter rate per formed binary $(\Lambda)$ is analyzed in conjunction with the $\gamma$-ray luminosity (Figure 4.5). The resulting insight is that if $\Lambda$ is very high, binary systems will get destroyed before having time to evolve into a MSP, so impacting the total number of MSPs in a GC.

- The characterization of a clean sample of $23 \gamma$-ray bright GCs, where 2 of them (M14 and M79) have never been reported before, as well as upper limits on energy flux for all remaining Milky Way GCs as listed in Harris (1996).

- No detected cluster presented extended emission; all of them are point-like sources spatially in agreement with the optical core of the GCs. We confirm (for the first time in $\gamma$-rays) the X-rays results of heavier objects sinking into the clusters' cores via dynamical friction.

\section{Acknowledgments}

We acknowledge useful discussions with Paulo C. C. Freire, Matthew Kerr, Philippe Bruel, Heitor Ernandes, Ana Chies Santos and Beatriz Barbuy. We thank the anonymous referee for constructive comments which helped to improve the manuscript. This work was supported by CNPq and FAPESP (Fundação de Amparo à Pesquisa do Estado de São Paulo, grants 2016/25484-9 and 2017/01461-2). The full acknowledgments can be found in the original paper by de Menezes et al. (2019). 


\subsection{Appendix: Upper limits on non-detected GCs and SEDs}

Energy flux upper limits with 95\% confidence levels and integrated over the whole analysis energy range are provided in Table 4.3, where the sources were assumed to have a power-law spectrum with a spectral index fixed at 2. A few of the sources described below are associated with globular clusters very close to the detection threshold in the preliminary LAT 8-year point source list ${ }^{6}$ (FL8Y) and will likely be part of the 4th FermiLAT catalog. The reason why these sources have TS slightly above the threshold in FL8Y is likely related to the different likelihood method used for creating the catalog (weighted likelihood).

The $\gamma$-ray spectra for 19 GCs, as discussed in Section 4.3.5, are shown in Figure 4.6. The spectra of only 4 of the 23 detected clusters were not included, all of them due to problems with low statistics. The clusters detected with high significance in $\gamma$-rays are mainly dominated by a logparabola spectral shape, while for the low-significance ones, the logparabola model is not statistically preferred over a power-law. The spectra of 2MASSGC01, Glimpse 01 and Glimpse 02 may have significant contamination from the Galactic diffuse emission, as these GCs are localized very close to the Galactic plane. The 19 spectra were obtained using the fermipy function $\operatorname{sed}()^{7}$, which computes the $\gamma$-ray spectra by performing independent fits for the flux normalization of a source in logarithmic spaced bins of energy (from $100 \mathrm{MeV}$ up to $100 \mathrm{GeV}$ in this case).

In section 4.3.6 a linear regression was performed taking into account only the measurements of $\Gamma$ and $N_{\text {MSP }}$ (cf. Fig. 4.3a). In order to quantify the impact of the non-detections on the results, the Python port of the LINMIX_ERR package ${ }^{8}$ was used. LINMIX_ERR is a Bayesian linear regression method that takes into account both measurement errors and non-detections (Kelly, 2007). The fit incorporating upper limits is given by

$$
\log N_{\mathrm{MSP}}=(2.17 \pm 0.55) \log \Gamma+(-2.86 \pm 1.07)
$$

which should be compared with the fit incorporating only measured values (cf. section 4.3.6),

$$
\log N_{\mathrm{MSP}}=(0.64 \pm 0.15) \log \Gamma+(0.80 \pm 0.20) .
$$

\footnotetext{
${ }^{6}$ https://fermi.gsfc.nasa.gov/ssc/data/access/lat/fl8y/

7 https://fermipy.readthedocs.io/en/latest/advanced/sed.html

8 https://github.com/jmeyers314/linmix
} 
Given that non-detections outnumber the detections by a factor of $\sim 7$, it is not surprising that the two fits are considerably different from each other, as can be seen in Figure 4.7. The fit involving only the detections is in good agreement with what is observed in X-rays and $\gamma$-rays for LMXBs and MSPs in previous works (Pooley et al., 2003; Abdo et al., 2010; Hooper and Linden, 2016), while the fit incorporating upper limits seems to underestimate the results in these works, giving a much lower number of MSPs per GC. This may be related to the high values of $\Lambda$ found for many of these non-detections, which may diminish the expected $\gamma$-ray flux even if the source has a large value for $\Gamma$ (see section 4.3.7 for details). 

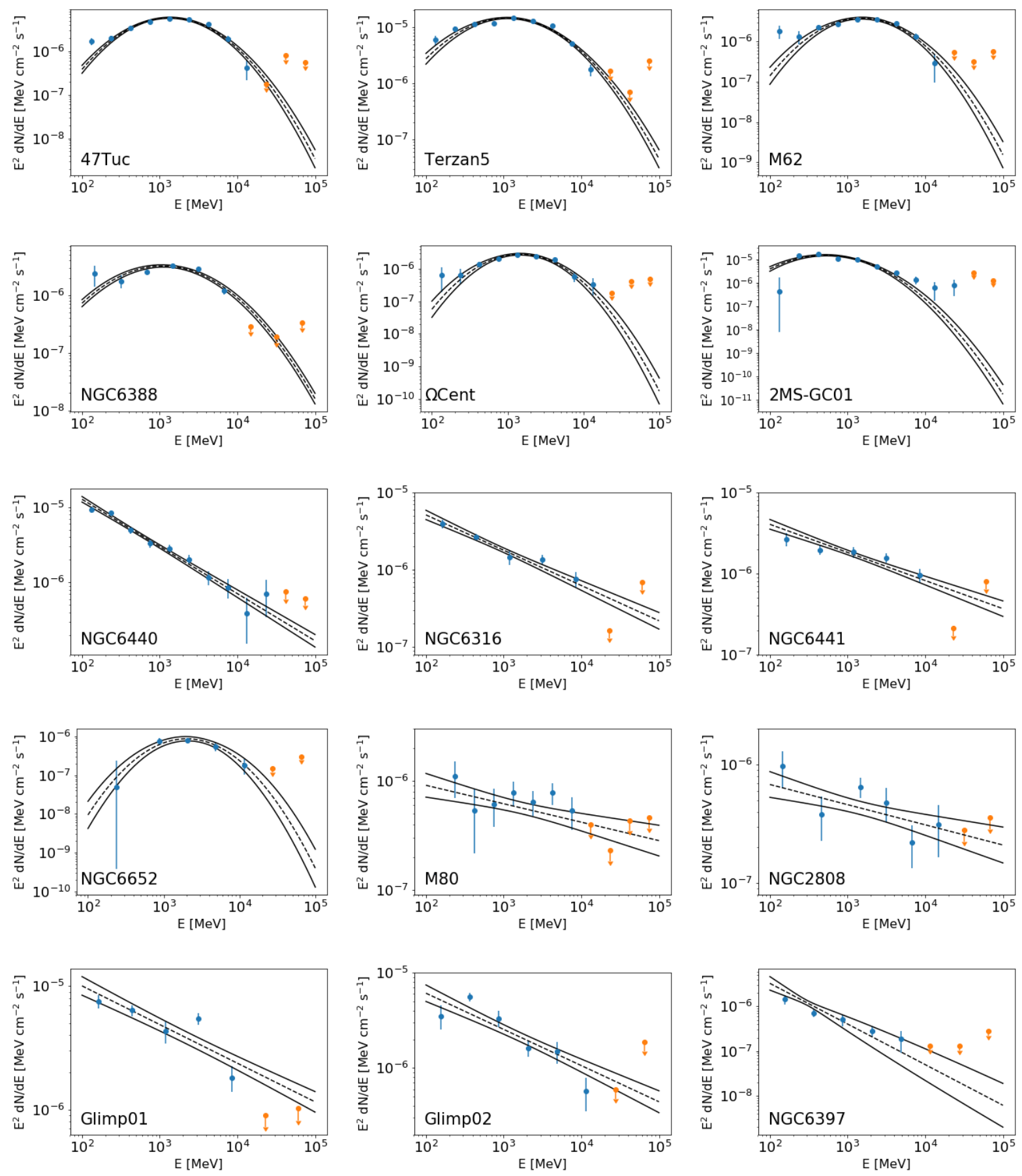

Figure 4.6: High energy $\gamma$-ray spectra for 19 of the $23 \gamma$-ray-bright GCs. The panels are organized accordingly with Table 4.1, with higher significance detections located in the top. All spectra are reasonably well fitted with a logparabola or power-law. It is easily noticeable the dominance of logparabola spectral shape between the most significant sources; the low-significance sources, on the other hand, are better described by a power-law. The adopted energy range was from $100 \mathrm{MeV}$ up to $100 \mathrm{GeV}$. 

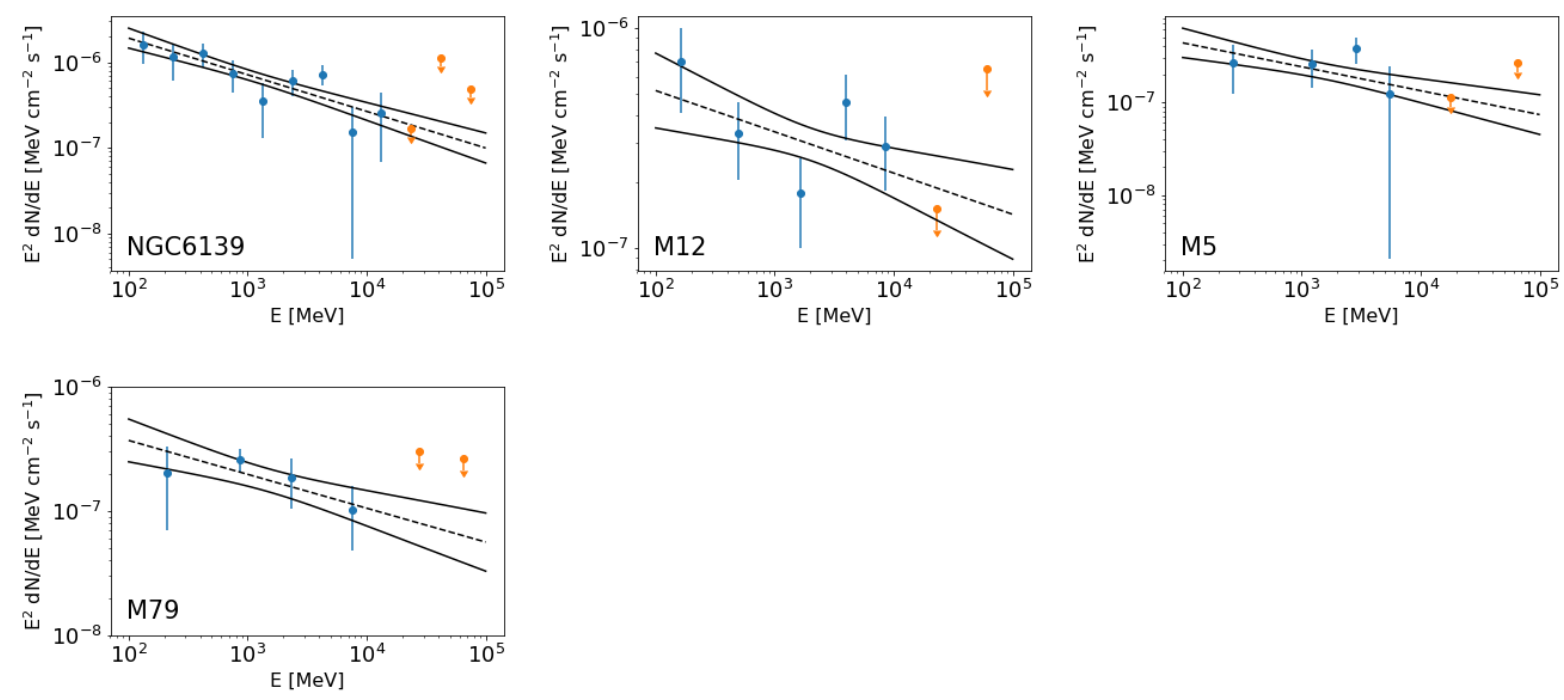

Figure 4.6: High energy $\gamma$-ray spectra for 19 of the $23 \gamma$-ray-bright GCs. The panels are organized accordingly with Table 4.1, with higher significance detections located in the top. All spectra are reasonably well fitted with a logparabola or power-law. It is easily noticeable the dominance of logparabola spectral shape between the most significant sources; the low-significance sources, on the other hand, are better described by a power-law. The adopted energy range was from $100 \mathrm{MeV}$ up to $100 \mathrm{GeV}$.

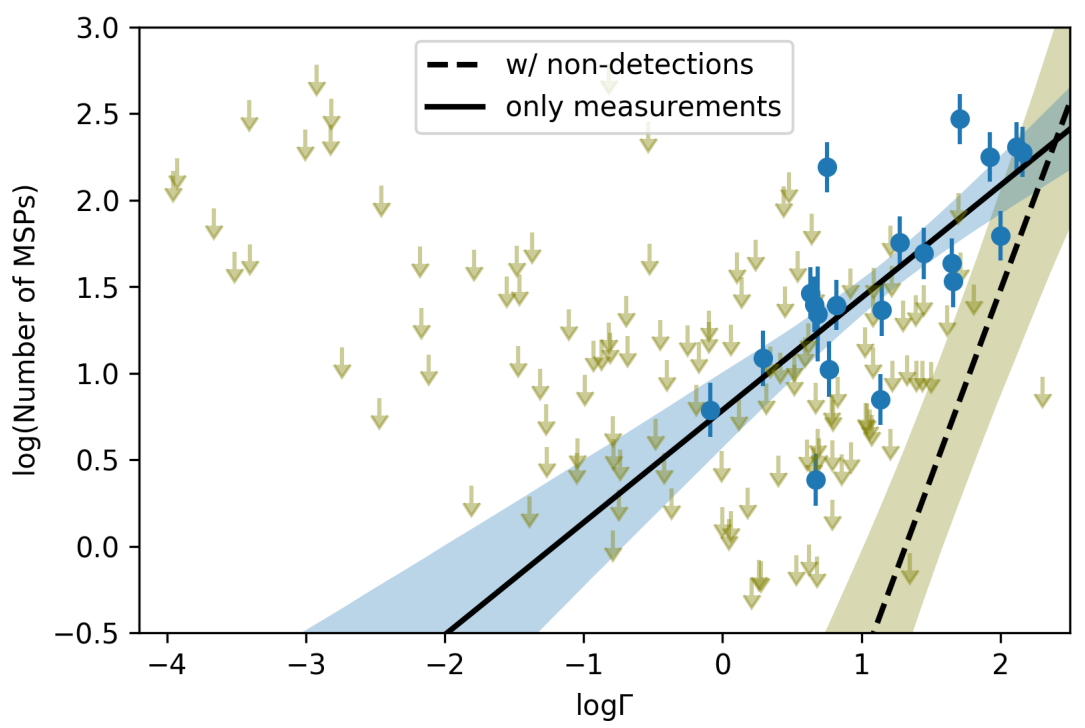

Figure 4.7: Linear regression for the number of MSPs and encounter rates. The solid line shows the fit to the data incorporating only actual measurements, while the dashed line takes into account measurements and non-detections (upper limits). The shaded regions around each line display the $1 \sigma$ credibility bands for each fit. 


\begin{tabular}{|c|c|c|}
\hline Cluster & $\begin{array}{c}\text { Upper limit } \\
10^{-13} \mathrm{erg} \mathrm{cm}^{-2} \mathrm{~s}^{-1}\end{array}$ & $\mathrm{TS}$ \\
\hline 2MASS-GC02 & 129.6 & 18 \\
\hline AM1 & 32.0 & 0 \\
\hline AM4 & 20.3 & 5 \\
\hline Arp2 & 8.2 & 1 \\
\hline BH176 & 18.2 & 1 \\
\hline BH261 & 6.0 & 0 \\
\hline Djorg1 & 7.6 & 0 \\
\hline Djorg2 & 26.4 & 3 \\
\hline E3 & 13.1 & 4 \\
\hline Eridanus & 5.7 & 0 \\
\hline ESO452-SC11 & 40.9 & 14 \\
\hline ESO-SC06 & 10.1 & 1 \\
\hline FSR1735 & 86.2 & 23 \\
\hline HP1 & 35.1 & 1 \\
\hline IC1257 & 41.1 & 14 \\
\hline IC1276 & 84.1 & 4 \\
\hline IC4499 & 22.2 & 7 \\
\hline Ko1 & 4.7 & 0 \\
\hline Ko2 & 1.7 & 0 \\
\hline Liller1 & 8.5 & 0 \\
\hline Lynga7 & 107.4 & 3 \\
\hline M2 & 6.1 & 0 \\
\hline M4 & 54.3 & 24 \\
\hline M9 & 1.7 & 0 \\
\hline M10 & 5.1 & 0 \\
\hline M13 & 32.3 & 23 \\
\hline$\ldots$ & $\ldots$ & $\cdots$ \\
\hline
\end{tabular}

Table 4.3 - Energy flux $2 \sigma$ upper limits and TS values for all 129 GCs non-detected in $\gamma$-rays. The energy range adopted is from $100 \mathrm{MeV}$ up to $100 \mathrm{GeV}$. The full version of this table can be found in de Menezes et al. (2019). 
Chapter 5

\section{The gamma-ray emission from LLAGNs}

This thesis chapter is a shortened version of the paper "Gamma-ray observations of low-luminosity active galactic nuclei" by de Menezes et al. (2020), published in the journal Monthly Notices of the Royal Astronomical Society, volume 492, number 3. The original work can be found online here.

\section{Abstract}

The majority of the activity around nearby $(z \approx 0)$ supermassive black holes is found in low-luminosity active galactic nuclei (LLAGN), the most of them being classified as low ionization nuclear emission regions. Although these sources are well studied from radio up to X-rays, they are poorly understood in $\gamma$-rays. In this work we take advantage of the all sky-surveying capabilities of the Large Area Telescope on board Fermi Gamma ray Space Telescope to study the whole Palomar sample of LLAGN in $\gamma$-rays. Precisely, the four radio-brightest LLAGN in the sample are identified as significant $\gamma$-ray emitters, all of which are recognized as powerful Fanaroff-Riley I galaxies. These results suggest that the presence of powerful radio jets is of substantial importance for observing a significant $\gamma$-ray counterpart even if these jets are misaligned with respect to the line of sight. We also find that most of the X-ray-brightest LLAGN do not have a significant $\gamma$-ray and strong radio emission, suggesting that the X-rays come mainly from the accretion flow in these cases. A detailed analysis of the spectral energy distributions (SEDs) of NGC 315 and NGC 4261, both detected in $\gamma$-rays, is provided where we make a detailed comparison between the predicted hadronic $\gamma$-ray emission from a radiatively inefficient accretion flow (RIAF) and the $\gamma$-ray emission from a leptonic jet-dominated synchrotron self-Compton 
(SSC) model. Both SEDs are better described by the SSC model while the RIAF fails to explain the $\gamma$-ray observations.

\subsection{Introduction}

Most active galactic nuclei (AGN) in the present day Universe are typified by low luminosities, being thus designated as low-luminosity AGN (LLAGN; e.g. Ho 2008). LLAGN consist of galaxies which host supermassive black holes (SMBHs) accreting with low, subEddington accretion rates $\dot{M}<0.01 \dot{M}_{\text {Edd }}$, where $\dot{M}_{\text {Edd }}$ is the Eddington accretion rate. Locally, some notable examples of LLAGN are Sagittarius A* - the SMBH at the Galactic center (Genzel et al., 2010) - and M87* at the center of the radio galaxy M87 (Event Horizon Telescope Collaboration et al., 2019). The bulk of the LLAGN population $(\sim 2 / 3)$ consists of low-ionization nuclear emission regions (LINERs; Halpern and Steiner, 1983) which have an average Eddington ratio of $L_{\mathrm{bol}} / L_{\mathrm{Edd}}=10^{-5}$ (Ho, 2009), where $L_{\mathrm{bol}}$ and $L_{\text {Edd }}$ are the bolometric and Eddington luminosities respectively.

The observational properties of LLAGN favor a scenario for their central engines which is quite different from that of more luminous AGN: since the SMBHs are accreting at low rates they are in the radiatively inefficient accretion flow (RIAF) mode (Ichimaru, 1977; Narayan and Yi, 1994a) rather than radiatively efficient geometrically thin accretion disks (Novikov and Thorne, 1973; Shakura and Sunyaev, 1973). Unlike standard thin disks (Shakura and Sunyaev, 1973) in which the viscously generated energy is thermalized and radiated locally, RIAFs store most of the viscous energy and advect it into the SMBH (Yuan and Narayan, 2014). The viscous heating affects mainly the ions, while the radiation is produced primarily by the electrons (Rees et al., 1982). Since the ions transfer only a small fraction of their energy to the electrons via Coulomb scattering, the energy which is radiated is much less than the total energy released during accretion (Rees et al., 1982). Due to the high temperatures and low densities, the leptonic and hadronic particle populations in RIAFs are expected to generate a broadband, multiwavelength electromagnetic spectrum from radio to $\gamma$-rays (Mahadevan et al., 1997; Oka and Manmoto, 2003).

LLAGN generally show compact radio core emission which is indicative of the presence of compact jets (Nagar et al., 2000, 2005). Furthermore, there is evidence that whenever LLAGN are observed at high angular resolutions, extended radio jet emission is detected 
(Mezcua and Prieto, 2014). Thus, observationally it seems that LLAGN are associated with relativistic jets. On the theoretical side, general relativistic magnetohydrodynamic simulations have demonstrated that RIAFs can easily produce powerful relativistic jets (Tchekhovskoy, 2015) provided that enough magnetic flux accumulates near the event horizon and the black hole spin is moderate at least (Nemmen et al., 2007; Sikora and Begelman, 2013). The presence of highly magnetized relativistic jets, carrying significant numbers of nonthermal particles (Petropoulou et al., 2016), introduces an important synchrotron radiation contribution (Spada et al., 2001) to the spectral energy distribution (SED).

The presence of both RIAFs and jets in LLAGN produces a rich multiwavelength SED (Yu et al., 2011; Nemmen et al., 2014; van Oers et al., 2017) in which a $\gamma$-ray component should be expected. There are multiple possible origins for the $\gamma$-ray emission. In the vicinity of the event horizon, the ion temperature of a RIAF can reach $10^{12} \mathrm{~K}$ (Yuan and Narayan, 2014), thus enabling proton-proton collisions and the production of neutral pions, which subsequently decay into pairs of GeV photons (Mahadevan et al., 1997; Oka and Manmoto, 2003). Furthermore, synchrotron self-Compton (SSC) emission is expected from the jet (Finke et al., 2008; Takami, 2011). Quantifying and modeling the $\gamma$-ray emission from LLAGN can give us valuable information on the relative importance of the contributions of the accretion flow and jet to the electromagnetic spectrum, the particle acceleration processes and particle populations responsible for the high-energy emission near the SMBHs.

The Fermi Large Area Telescope (LAT) has been observing the whole sky for over ten years in the $100 \mathrm{MeV}$ to $>300 \mathrm{GeV}$ energy band (Atwood et al., 2009). The excellent sky coverage, broad spectral range and time-domain resolution of Fermi-LAT has brought a revolution in our understanding of the extragalactic $\gamma$-ray sky (Massaro et al., 2016). Naturally, in the last few years progress has been made on the observational search for $\gamma$-rays coming from LLAGN as well as Seyfert 1 AGN, which we now review.

Using 3 years of Fermi-LAT observations, Ackermann et al. (2012) performed a search for $\gamma$-rays in a sample of 120 radio-quiet Seyferts, selected based on the presence of hard X-ray emission from the Neil Gehrels Swift Observatory Burst Alert Telescope (BAT) 58 month catalog (Barthelmy et al., 2005; Cusumano et al., 2010). Ackermann et al. (2012) found no significant $\gamma$-ray emission in Seyfert galaxies that can be attributed to the AGN. 
Teng et al. (2011) used 2.1 years of Fermi-LAT data to search for $\gamma$-rays from Seyferts selected from the same catalog used by Ackermann et al. (2012) and found that the radioloud AGN-mostly blazars-were detected by Fermi-LAT whereas only two radio-quiet AGN are $\gamma$-ray emitters, NGC 1068 and NGC 4945. Wojaczyński et al. (2015) searched for $\gamma$ ray emission in a sample of 11 prominent Seyfert 1s including the Fanaroff-Riley type I (FRI) galaxy Centaurus A. Wojaczynski et al. found significant $\gamma$-ray detection in NGC 6814, and weak signals associated with NGC 4151 and NGC 4258 which could be due to background emission.

Another interesting non-blazar class of objects observed with Fermi-LAT are radio galaxies with their jets pointing away from us, so-called misaligned AGN (Abdo et al., 2010)-many of which host LLAGN. They are radio-loud sources with steep radio spectra $\left(\alpha_{r}>0.5\right.$, in the convention $\left.F_{\nu} \propto \nu^{-\alpha_{r}}\right)$. Observations of these objects in $\gamma$-rays were previously obtained at $\mathrm{GeV}$ and even TeV energies (Abdo et al., 2009; Fermi LAT Collaboration, 2010a; Abdo et al., 2010; Grandi et al., 2013; Aleksic et al., 2014; Aleksić et al., 2014; Abdalla et al., 2018). Since the emission from misaligned AGN is not as affected by relativistic boosting due to their misaligned jets, in contrast with blazars, their $\gamma$-ray emission could potentially have a hadronic contribution from the RIAF. The observed $\gamma$ ray SEDs, however, indicate a dominance of leptonic emission, most likely SSC emission, rather than a hadronic process (Abdo et al., 2009; Fermi LAT Collaboration, 2010a).

The observed $\gamma$-ray properties of LLAGN as a class has not been systematically explored yet. In this work we make the first systematic investigation of the Fermi-LAT observations of LLAGN as a class, and we compare the observations with predictions from RIAF and jet models for the $\gamma$-ray emission. Throughout this work, we use a Hubble constant $H_{0}=75$ $\mathrm{km} \mathrm{s}^{-1} \mathrm{Mpc}^{-1}$, to be consistent with Nagar et al. (2005) and Ho (2009), who tabulated the radio and X-ray observations of the Palomar sample. The definition of LLAGN we adopt is based on the optical nuclear spectra of the sources which are generally LINERs. Some of these sources, however, can be very bright in other wavelengths, especially in radio. This paper is structured as follows. Sections 5.2 and 5.3 describe the sample, data selection and the $\gamma$-ray, X-ray and radio analyses. The theoretical models for the electromagnetic spectrum are presented in Section 5.4. The results are presented in Section 5.5 and discussed in Section 5.6. We finish with a summary of the work and conclusions in Section 5.7. 


\subsection{Fermi-LAT observations}

Our sample consists of the 197 AGN found among the 486 bright (apparent blue magnitudes $B_{T} \leq 12.5 \mathrm{mag}$ ) northern (declination $\delta>0^{\circ}$ ) galaxies in the Palomar spectroscopic survey of nearby galactic nuclei (Ho et al., 1995; Ho et al., 1997). This sample is characterized by high-quality, moderate-resolution, long-slit spectra with a high completeness degree due to the bright magnitude limit adopted. The properties of the Palomar survey make it suitable to the study of nearby $(z \approx 0)$ AGN, especially LLAGN (Ho, 2008).

For each AGN in our sample we analyzed data collected with Fermi-LAT during a period of 10.25 years ranging from August 4th 2008 to November 15th 2018. The analysis was performed with fermitools ${ }^{1}$ v1.0.0 and fermipy ${ }^{2}$ v0.17.4 (Wood et al., 2017), as well as the Pass 8 event processed data (Atwood et al., 2013).

Following the recommended criteria ${ }^{3}$, we selected data within a $15^{\circ} \times 15^{\circ}$ region-ofinterest (ROI), centered on the AGN positions given in Ho et al. (1995), with energies ranging between $100 \mathrm{MeV}$ and $300 \mathrm{GeV}$ and 8 logarithmically spaced bins per energy decade. We then manually added a point source to the center of each ROI, except when the target was already available in the Fermi-LAT 8-year Source Catalog ${ }^{4}$ (4FGL; Abdollahi et al., 2020).

Only events belonging to the Source class have been used (evclass=128 and evtype=3) and the filters applied with gtmktime were DATA_QUAL $>0$ and the recommended instrument configuration for science (LAT_CONFIG==1). We applied a maximum zenith angle cut of $90^{\circ}$ to reduce contamination from the Earth limb. For modeling the Galaxy and the extragalactic background emission, we adopted the Galaxy background model gll_iem_v07 and the isotropic spectral template iso_P8R3_SOURCE_V2_v1.

We investigated these AGN by means of binned likelihood analysis using MINUIT as minimizer and, to quantify the significance among the detections, we used a test statistic (TS) defined as $T S=2\left(\mathcal{L}_{1}-\mathcal{L}_{0}\right)$, where the term inside parentheses is the difference between the maximum log-likelihoods with $\left(\mathcal{L}_{1}\right)$ and without $\left(\mathcal{L}_{0}\right)$ including a point source with power-law photon index $\alpha=2$ in the model. The adopted criteria for detection was

\footnotetext{
${ }^{1}$ https://fermi.gsfc.nasa.gov/ssc/data/analysis/software/

2 https://fermipy.readthedocs.io/en/latest/index.html

3 https://fermi.gsfc.nasa.gov/ssc/data/analysis/documentation/Cicerone/Cicerone_Data_ Exploration/Data_preparation.html

${ }^{4}$ https://fermi.gsfc.nasa.gov/ssc/data/access/lat/8yr_catalog/
} 


\begin{tabular}{l|c|c|c|c|c|c} 
Source & TS & $\mathrm{TS}_{\text {comp }}$ & $\Gamma$ & $\Gamma_{\text {comp }}$ & $\begin{array}{c}\text { Energy flux } \\
10^{-12} \mathrm{erg} \mathrm{cm}^{-2} \mathrm{~s}^{-1}\end{array}$ & $\begin{array}{c}\text { Energy flux } \\
10^{-12} \mathrm{erg} \mathrm{cm}^{-2} \mathrm{~s}^{-1}\end{array}$ \\
\hline NGC 315 & 90.09 & 90.66 & $2.32 \pm 0.11$ & $2.33 \pm 0.11$ & $3.38 \pm 0.43$ & $3.40 \pm 0.43$ \\
NGC 4261 & 46.22 & 47.80 & $2.15 \pm 0.16$ & $2.12 \pm 0.15$ & $2.15 \pm 0.42$ & $2.19 \pm 0.43$ \\
NGC 4374 & 15.01 & 15.49 & $2.05 \pm 0.20$ & $2.08 \pm 0.21$ & $1.09 \pm 0.38$ & $1.12 \pm 0.38$ \\
NGC 4151 & 11.05 & 13.05 & $1.99 \pm 0.29$ & $2.14 \pm 0.28$ & $0.72 \pm 0.30$ & $0.83 \pm 0.32$
\end{tabular}

Table 5.1 - Comparison between the values obtained in the main analysis and in the complementary analysis (tagged with the subscript). The complementary analysis was computed with an extra iteration with DRMNFB minimizer and with all sources within a circle with $7^{\circ}$ radius from the ROI center free to vary. The results are in agreement with the main analysis within the error bars.

TS $>25$, corresponding to a significance of $5 \sigma$ (Mattox et al., 1996). For all sources lying within a radius of $5^{\circ}$ from the center of the ROIs, the normalization parameter was left free to vary.

All sources listed in 4FGL and lying up to $5^{\circ}$ outside the ROIs have been taken into account as well as all sources found with the fermipy function find_sources () using the parameters sqrt_ts_threshold=5.0 and min_separation=0.5. Three of the 197 AGN analyzed (NGC 315, NGC 1275 and NGC 4486) belong to 4FGL and thus we modelled their spectral shape accordingly to the catalog. These three sources together with NGC 4261 were the only AGN detected in $\gamma$-rays (TS $>25$; see Section 5.5). All remaining sources were modelled with a power-law spectrum and their flux upper limits are listed in Appendix 5.8.

To test the robustness of our analysis, we performed a complementary analysis from scratch only for the LLAGN with TS values close to the adopted detection threshold of $\mathrm{TS}=25$. A total of four sources were selected within the range $10<\mathrm{TS}<100$. This time we applied an extra iteration with DRMNFB minimizer (before using MINUIT) and let the normalization and spectral shape of all sources within a radius of $7^{\circ}$ from the ROI center free to vary. The results of this complementary analysis are in agreement with the results of the main analysis of this work and can be checked in Table 5.1.

\subsection{X-ray and radio data}

\subsubsection{X-rays}

The X-ray observations we adopted are from Ho (2009), where he performed a literature search and collected all published X-ray observations for the LLAGN in the Palomar sample. Because most nearby AGN are very dim in X-rays, they can be easily outshi- 
ned by circumnuclear emission. Hence, the most important consideration when selecting X-ray observations in the literature is the angular resolution, in order to avoid possible contamination from other sources not associated with the AGN. Most of the observations (about 75\%) were acquired with Chandra/ACIS, and the rest of them with ROSAT/HRI or XMM-Newton, with resolutions between $1^{\prime \prime}-5^{\prime \prime}$ which is acceptable for our purposes. This selection resulted in X-ray data for 175 LLAGN. As different instruments have been used with different techniques, all X-ray luminosities were converted to the standard bandpass of 2-10 keV using the best fit spectral slope available in the literature. When not available, a photon index of $\Gamma=1.8$ was assumed. The details of the X-ray analysis are described in Ho (2009). Among the four AGN detected in $\gamma$-rays in this work (see Section 5.5), NGC 315, NGC 4261 and NGC 4486 were observed with Chandra, while NGC 1275 was observed with XMM-Newton.

\subsubsection{Radio}

The radio data we adopted are from the Very Large Array (VLA) survey of LLAGN by Nagar et al. $(2000,2005)$ with angular resolution of $\sim 0.15^{\prime \prime}$. All but four of the Palomar sample LLAGN have been observed at sub-parsec resolution with the VLA at $15 \mathrm{GHz}$. The exceptions are NGC 5850, NGC 5970, NGC 5982 and NGC 5985 and none of them are expected to be detected in the radio survey, as their measured fluxes at 1.4-5 GHz with $1^{\prime \prime}-5^{\prime \prime}$ resolution are less than 1 mJy (Wrobel and Heeschen, 1991).

\subsection{Spectral models}

In order to make sense of the best-sampled multiwavelength broadband SEDs among our targets and contrast different possible origins for the electromagnetic emission, we used two different models. In the first model, we assumed that the emitting particles are located in a relativistic jet while in the second one the emission comes from the RIAF, as appropriate for sub-Eddington LLAGN.

The jet model consists of a spherical blob moving at relativistic speeds filled with nonthermal electrons - the usual homogeneous one-zone SSC model (Finke et al., 2008) which has been successfully applied to explain the SEDs of several radio galaxies observed with the LAT (Fermi LAT Collaboration, 2010a; Aleksic et al., 2014). The blob moves 
with a bulk Lorentz factor $\Gamma_{j}=(1-\beta)^{-1 / 2}$ (where the jet speed is $\beta c$ ) at an angle to the line of sight $\theta$. This gives a Doppler factor $\delta_{D}=\left[\Gamma_{j}(1-\beta \cos \theta)\right]^{-1}$. We use a broken power-law electron distribution,

$$
N_{e}(\gamma) \propto \begin{cases}0 & \gamma<\gamma_{\text {min }} \\ \gamma^{-p_{1}} & \gamma<\gamma_{\text {break }} \\ \gamma^{-p_{2}} & \gamma_{\text {break }}<\gamma \\ 0 & \gamma_{\max }<\gamma\end{cases}
$$

where $\gamma$ is the electron Lorentz factor and $p_{1}, p_{2}, \gamma_{\text {break }}, \gamma_{\min }$, and $\gamma_{\max }$ are free parameters. Other parameters for this model include the comoving blob radius $R_{b}$ and the comoving magnetic field strength $B$. We adopted the parameters $\Gamma_{j}=1.5, \gamma_{\min }=1.0$ and $p_{2}=3.0$ (see e.g., Finke, 2013).

For the RIAF emission model, we used a semi-analytical approach to treat the radiation from the accretion flow in which its structure is considered stationary assuming an $\alpha$ viscosity and a pseudo-Newtonian gravity appropriate for a Schwarzschild black hole, while the radiative transfer is treated in considerable detail (Nemmen et al., 2006, 2014). For simplicity, when modeling the RIAF emission we do not consider the contribution to the emission by an optically thick, geometrically thin accretion disk. The main parameters of this model are described below. We incorporated the presence of mass loss from the accretion flow through winds by modifying the RIAF density profile, with the parameter $s$ describing the radial variation of the accretion rate as $\dot{M}(R)=\dot{M}_{\mathrm{o}}\left(R / R_{\mathrm{o}}\right)^{s}($ or $\rho(R) \propto$ $R^{-3 / 2+s}$ ) where $\dot{M}_{\mathrm{o}}$ is the rate measured at the outer radius $R_{\mathrm{o}}$ of the RIAF (Blandford and Begelman, 1999). The other parameters that describe the RIAF solution are the black hole mass $M_{\bullet}$; the viscosity parameter $\alpha$; the modified plasma $\beta$ parameter, defined as the ratio between the gas and total pressures, $\beta=P_{g} / P_{\text {tot }}$; the fraction of energy dissipated via turbulence that directly heats electrons $\delta$; and the adiabatic index $\gamma_{a}$. Following Nemmen et al. (2014), in our calculations we adopted the typical choice of parameters $\alpha=0.3$, $\beta=0.9, \gamma_{a}=1.5$ and $R_{o}=10^{4} R_{S}$.

One novelty in this work is that in addition to computing the electromagnetic radiation spectrum of electrons which can explain the observed radio-to-X-ray emission (Almeida et al., 2018), we also take into account the contribution of the proton population. Incorporating hadronic emission is essential in order to be able to model the Fermi-LAT 
observations and assess whether they can be explained by a RIAF scenario.

For the leptonic part, we considered the synchrotron, inverse Compton scattering and bremsstrahlung processes due to a thermal distribution of electrons. For the hadronic part, the flow temperatures are so high (see Yuan and Narayan, 2014) that proton-proton collisions create neutral pions which subsequently decay into $\gamma$-rays (Mahadevan et al., 1997). We considered the presence of a small fraction of nonthermal protons following a power-law energy distribution which is properly normalized following Mahadevan et al. (1997). The pion decay spectrum itself was calculated using Naima ${ }^{5}$ v0.8.3 (Zabalza, 2015), which uses a parametrization for the integral cross-section, pion production rates and photon energy spectra from p-p interactions based on Monte Carlo simulations (Kafexhiu et al., 2014). A power-law index of 2.3 was assumed for the proton energy distribution function, consistent with general expectations of particle acceleration theories (Sironi and Spitkovsky, 2014).

We now describe our modeling approach for the synchrotron/SSC one-zone jet and the RIAF. For a given target, we first searched the literature for important empirical priors for our modeling such as the black hole mass and jet inclination angle - which is relevant only for the jet model since the RIAF emission is roughly isotropic - and fixed the values of those parameters in the models to the measured ones.

For the jet model, we fixed the jet angle $\theta$ to the values available in Canvin et al. (2005) and Piner et al. (2001) for NGC 315 and NGC 4261, respectively. Part of the radio emission was considered to contribute as upper limits to the one-zone emission, as it likely comes from a larger region than the one responsible for the rest of the multiwavelength emission. For both NGC 315 and NGC 4261 it was not possible to fit the X-ray together with all of the $\gamma$-ray emission with a single SSC component from the same population of electrons (see Section 5.5).

We divided the RIAF modeling process in two parts. In the first, we allowed the parameters $\dot{M}_{o}, s$ and $\delta$ to vary in an iterative way, where we changed individually the parameters, keeping the others fixed, until we find the set of values that best reproduces visually the mm-to-X-ray observations, as previously done in other works (Almeida et al., 2018). These bands comprise the synchrotron and inverse Compton emission of the leptonic population. Once we have fixed the parameters using the leptonic model, they determine

\footnotetext{
${ }^{5}$ https://github.com/zblz/naima
} 
the radial structure of the accretion flow such as the electron and proton number density and temperature. As a post-processing step, we used the proton radial profiles as inputs to calculate the $\gamma$-ray contribution of the hadronic population.

\subsection{Results}

The four AGN detected with TS $>25$ by the Fermi-LAT - i.e. with significant $\gamma$-ray emission - are listed in Table 5.2 along with their radio, X-ray and $\gamma$-ray luminosities, and spectral type. They are all known radio galaxies, including the familiar $\gamma$-ray emitters M87 and NGC 1275 (Abdo et al., 2009; Nemmen et al., 2018).

\begin{tabular}{lccccccc} 
Name & type & Dist. $(\mathrm{Mpc})$ & $\log \left(L_{15 G H z}\right)$ & $\log \left(L_{x}\right)$ & $\log \left(L_{\gamma}\right)$ & $\Gamma$ & TS \\
\hline NGC 1275 & Seyfert 1.5 & 70 & 41.42 & 42.86 & $44.30 \pm 0.01$ & $2.10 \pm 0.01$ & 132350 \\
NGC 4486 & LINER 2 & 16 & 40.14 & 40.78 & $41.83 \pm 0.02$ & $2.04 \pm 0.03$ & 1629 \\
NGC 315 & LINER 1.9 & 65 & 40.57 & 41.63 & $42.20 \pm 0.06$ & $2.32 \pm 0.11$ & 90 \\
NGC 4261 & LINER 2 & 35 & 39.83 & 40.59 & $41.50 \pm 0.08$ & $2.15 \pm 0.16$ & 46
\end{tabular}

Table 5.2 - The 4 AGN in the Palomar sample detected in $\gamma$-rays. The columns give the name, nuclear spectral type, distance, the radio, X-ray and $\gamma$-ray luminosities in erg/s, the $\gamma$-ray power-law spectral index $(\Gamma)$ and the TS. Radio and X-ray data were taken from Nagar et al. (2005) and Ho (2009), respectively. The distances adopted for calculating the $\gamma$-ray luminosities, assumed as isotropic, were taken from Nagar et al. (2005)

Figure 5.1 shows the relation between the radio and $\gamma$-ray luminosities for the sample. Interestingly, the four nuclei detected in $\gamma$-rays are also the radio-brightest objects in the Palomar sample, corresponding to FRI radio galaxies (Fanaroff and Riley, 1974; Venturi et al., 1993; Sambruna et al., 2003; Nagar et al., 2005).

In order to assess any possible correlation between the $\gamma$-ray and radio luminosities in Figure 5.1 in the presence of data with left-censoring (upper limits), we have performed two different tests: the Cox's regression based on the proportional hazard model (Cox, 1972; Isobe et al., 1986) and the Akritas-Thiel-Sen (ATS) Kendall $\tau$-rank correlation test (Feigelson and Babu, 2012). We performed Cox's regression using the ASURV package (Lavalley et al., 1992) and obtain the probability $P_{\text {Cox }}=0.03$ that no correlation exists. Following Feigelson and Babu (2012), we computed the ATS Kendall $\tau$ correlation coefficient using $\mathrm{R}$ and the $\mathrm{NADA}^{6}$ package, finding $\tau=0.04$ with the associated probability $P_{\mathrm{ATS}}=0.4$ that no correlation is present. The two methods give conflicting results, with the ATS method giving more conservative results and suggesting no correlation whereas

\footnotetext{
${ }^{6}$ https : //CRAN.R-project.org/package=NADA
} 


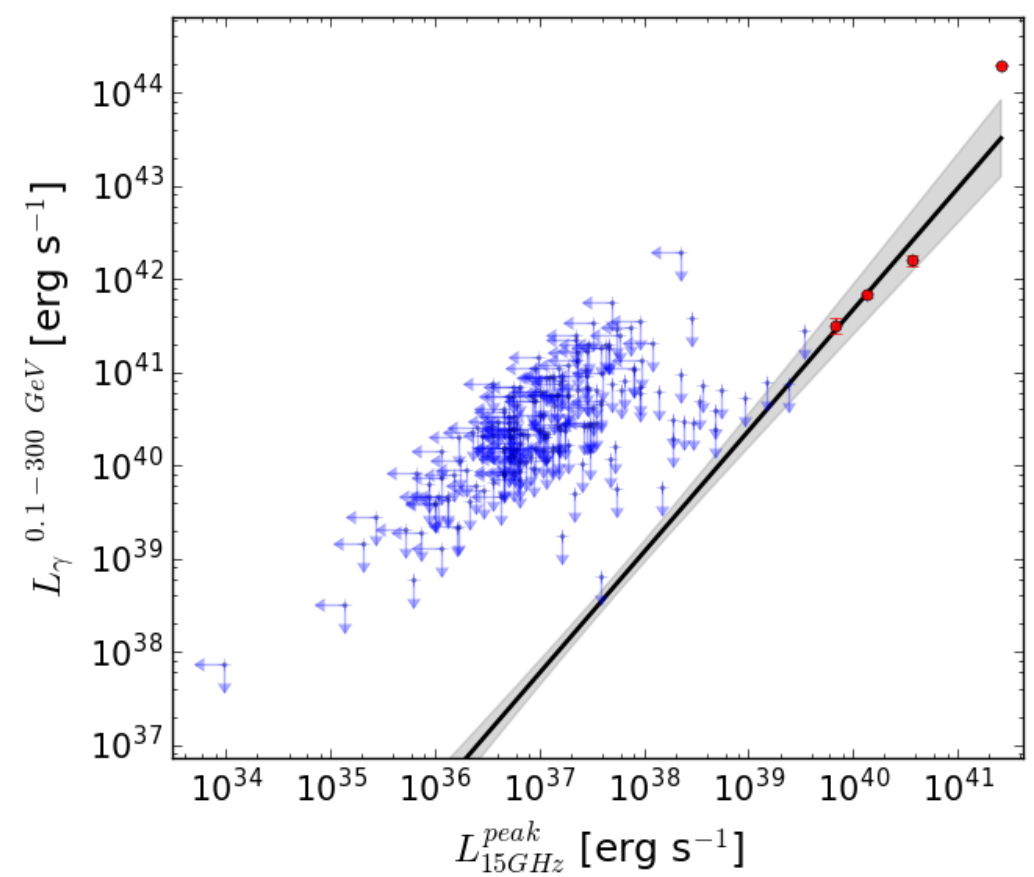

Figure 5.1: The $\gamma$-ray luminosity $(100 \mathrm{MeV}-300 \mathrm{GeV})$ as a function of radio luminosity (15 GHz) for the AGN in the Palomar sample. The arrows indicate upper limits. The filled red circles indicate the significant detections in $\gamma$-rays. The solid line shows the linear regression fit to the data taking into account measurements and non-detections (upper limits). The shaded regions around the line display the $1 \sigma$ credibility bands for each fit. All values for upper limits are available in Appendix 5.8.

the Cox's method suggests a possible correlation with low significance (null hypothesis can be rejected at the $2.1 \sigma$ level). We conclude that there is a weak evidence for a $\gamma$-ray-radio correlation considering all upper limits.

For the fit in Figure 5.1 we used the Bayesian linear regression method LINMIX_ERR package $^{7}$ that takes into account both measurement errors and non-detections (Kelly, 2007). The fit gives:

$$
\log L_{\gamma}=(1.30 \pm 0.12) \log L_{\text {radio }}+(-10.33 \pm 4.61)
$$

Even though the slope of the linear regression is large, one should keep in mind the low significance of the radio- $\gamma$-ray correlation as obtained from the nonparametric Cox and ATS methods.

Figure 5.2 shows the relation between the $\gamma$-ray and X-ray $(2-10 \mathrm{keV})$ luminosities for the sample. Interestingly, most of the brightest X-ray AGN have not been detected in $\gamma$-rays, which may indicate that most of the X-ray emission in these $\gamma$-ray-faint nuclei is probably not powered by a jet (Markoff et al., 2001, 2003), but instead coming from the accretion flow itself (Veledina et al., 2013). This hypothesis is reinforced by the fact

\footnotetext{
7 https://github.com/jmeyers314/linmix
} 


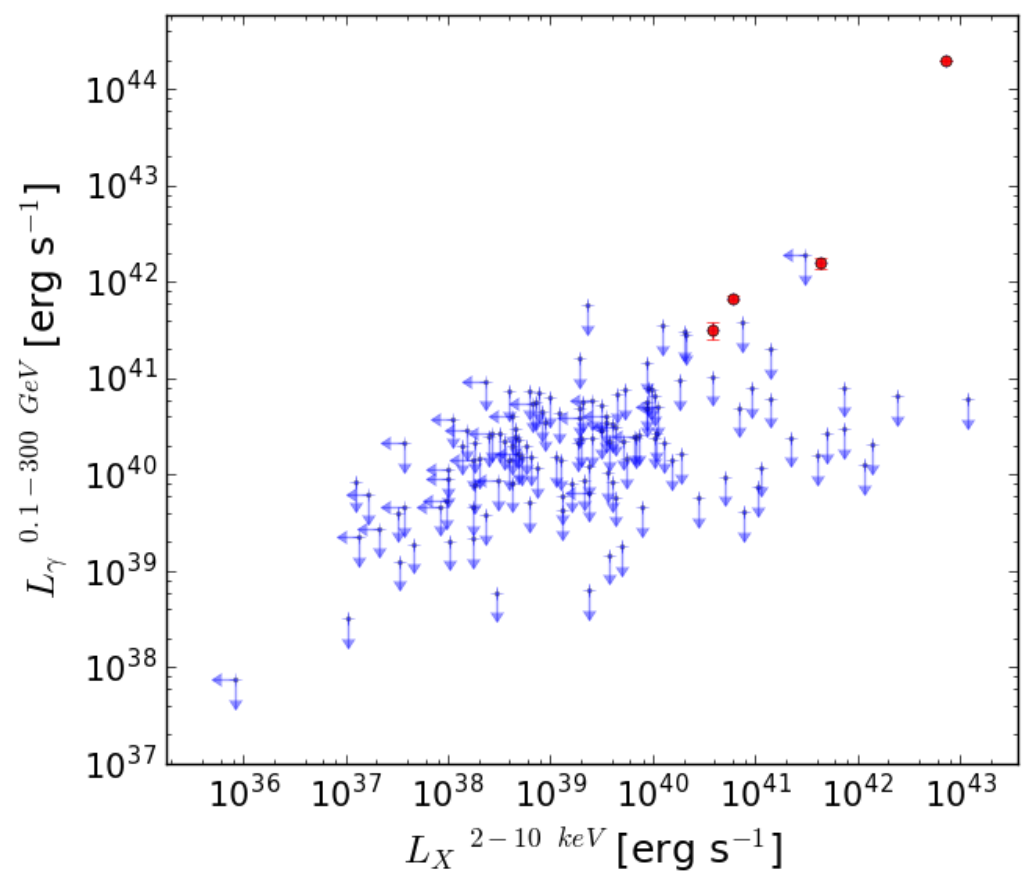

Figure 5.2: The $\gamma$-ray luminosity as a function of the X-ray luminosity $(2-10 \mathrm{keV})$ for the AGN in the Palomar sample. Most bright X-ray AGN are not detected in $\gamma$-rays.

that most of these X-ray-bright AGN are relatively faint in radio (Nagar et al., 2005). Applying the Cox and ATS correlation tests, we find $P_{\text {Cox }}=0.3$ and $P_{\text {ATS }}=0.4$ taking into account all upper limits. Therefore, there is no correlation between $L_{\gamma}$ and $L_{X}$ in the sample except perhaps for the $\gamma$-ray-bright sources (red points in Figure 5.2). Indeed, these four AGN are known by having powerful jets or bubbles resolved in X-rays (Young et al., 2002; Gonzalez-Martin et al., 2006; Worrall et al., 2010; Fabian et al., 2015), thus meaning that their jets can significantly contribute to their X-ray emission.

In order to probe the nature of the observed Fermi-LAT emission in our samplei.e. do the $\gamma$-rays originate in the jet or in the accretion flow? - we explored the two models described in section 5.4: jet and RIAF. The radiation in the jet model is purely of leptonic origin while in the RIAF it is a combination of leptonic and hadronic processes. We proceed by comparing their radio-to- $\gamma$-rays SEDs with the two models. We would like to emphasize that the adopted multiwavelength data are not simultaneous, with the radio-to-X-ray observations being collected from 1996 up to 2014 (see references in Table 5.3), making it difficult to consistently fit the whole SED with a single model.

Two of the strong $\gamma$-ray sources have already been well-explained in terms of synchrotron/SSC jet models: M87 and NGC 1275 (Abdo et al., 2009; Tanada et al., 2018). For this reason, we choose to perform a detailed modeling for the other two sources, NGC 315 


\begin{tabular}{lcc}
\hline$\nu(\mathrm{Hz})$ & $\nu L_{\nu}\left(\mathrm{erg} \mathrm{s}^{-1}\right)$ & Reference \\
\hline $1.40 \mathrm{E}+09$ & $2.86 \mathrm{E}+39$ & $\mathrm{a}$ \\
$2.50 \mathrm{E}+09$ & $5.28 \mathrm{E}+39$ & $\mathrm{~b}$ \\
$5.00 \mathrm{E}+09$ & $9.11 \mathrm{E}+39$ & $\mathrm{c}$ \\
$1.50 \mathrm{E}+10$ & $3.68 \mathrm{E}+40$ & $\mathrm{c}$ \\
$8.62 \mathrm{E}+10$ & $(2.28 \pm 0.13) \mathrm{E}+41$ & $\mathrm{~d}$ \\
$2.29 \mathrm{E}+11$ & $(3.08 \pm 0.23) \mathrm{E}+41$ & $\mathrm{~d}$ \\
$3.75 \mathrm{E}+13$ & $(1.47 \pm 0.08) \mathrm{E}+42$ & $\mathrm{e}$ \\
$5.17 \mathrm{E}+13$ & $(7.72 \pm 0.59) \mathrm{E}+41$ & $\mathrm{e}$ \\
$6.67 \mathrm{E}+13$ & $(4.63 \pm 0.45) \mathrm{E}+41$ & $\mathrm{e}$ \\
$8.33 \mathrm{E}+13$ & $(2.95 \pm 0.35) \mathrm{E}+41$ & $\mathrm{e}$ \\
$3.68 \mathrm{E}+14$ & $2.00 \mathrm{E}+41$ & $\mathrm{f}$ \\
$5.40 \mathrm{E}+14$ & $9.48 \mathrm{E}+40$ & $\mathrm{f}$ \\
$(4.8-24) \mathrm{E}+17$ & $4.36 \mathrm{E}+41$ & $\mathrm{~g}$ \\
\hline $1.63 \mathrm{E}+09$ & $2.40 \mathrm{E}+38$ & $\mathrm{~h}$ \\
$5.00 \mathrm{E}+09$ & $5.88 \mathrm{E}+38$ & $\mathrm{c}$ \\
$8.39 \mathrm{E}+09$ & $1.24 \mathrm{E}+39$ & $\mathrm{~h}$ \\
$1.50 \mathrm{E}+10$ & $6.71 \mathrm{E}+39$ & $\mathrm{c}$ \\
$1.66 \mathrm{E}+13$ & $(5.39 \pm 0.24) \mathrm{E}+41$ & $\mathrm{i}$ \\
$2.50 \mathrm{E}+13$ & $(4.79 \pm 1.10) \mathrm{E}+41$ & $\mathrm{i}$ \\
$3.64 \mathrm{E}+14$ & $6.03 \mathrm{E}+39$ & j \\
$4.41 \mathrm{E}+14$ & $4.60 \mathrm{E}+39$ & j \\
$5.41 \mathrm{E}+14$ & $3.96 \mathrm{E}+39$ & $\mathrm{j}$ \\
$(4.8-24) \mathrm{E}+17$ & $1.03 \mathrm{E}+41$ & $\mathrm{k}$ \\
\hline
\end{tabular}

Table 5.3 - SED data for NGC 315 (top section) and NGC 4261 (bottom section). The references are ${ }^{a}$ Capetti et al. (2005), ${ }^{b}$ Lazio et al. (2001), ${ }^{\mathrm{c}}$ Nagar et al. (2005), ${ }^{\mathrm{d}}$ Agudo et al. (2014), ${ }^{\mathrm{e}} \mathrm{Gu}$ et al. (2007), ${ }^{\mathrm{f}}$ Verdoes Kleijn et al. (2002), ${ }^{\mathrm{g}}$ Gonzalez-Martin et al. (2006), ${ }^{\mathrm{h}}$ Jones and Wehrle (1997), ${ }^{\mathrm{i}}$ Asmus et al. (2014), ${ }^{\mathrm{j}}$ Ferrarese et al. (1996) and ${ }^{\mathrm{k}}$ Zezas et al. (2005).

and NGC 4261. The jet inclinations were $38^{\circ} \pm 2^{\circ}$ (NGC 315, Canvin et al. 2005) and $63^{\circ} \pm 3^{\circ}$ (NGC 4261, Piner et al. 2001), respectively. All data points used to assemble the SEDs correspond to the core emission. The infrared data is likely contaminated by dust emission, so the observations in this band are treated as upper limits to the jet emission.

The result from modeling the SED of NGC 315 with the jet scenario is displayed in the left panel of Figure 5.3. The X-ray spectrum and the lowest LAT energy bins $(E<5$ $\mathrm{GeV}$ ) are adequately reproduced with a SSC component. However, the highest energy LAT emission $(E>5 \mathrm{GeV})$ cannot be explained by jet SSC emission and could be from yet another component which we have not taken into account. The jet model parameters are displayed in Table 5.4. The model fitted to the data is not rigorous, but rather obtained through visual inspection and iterative calculations, and the values of the parameters in Table 5.4 are not unique. Another feature of the jet model is that it underpredicts the 

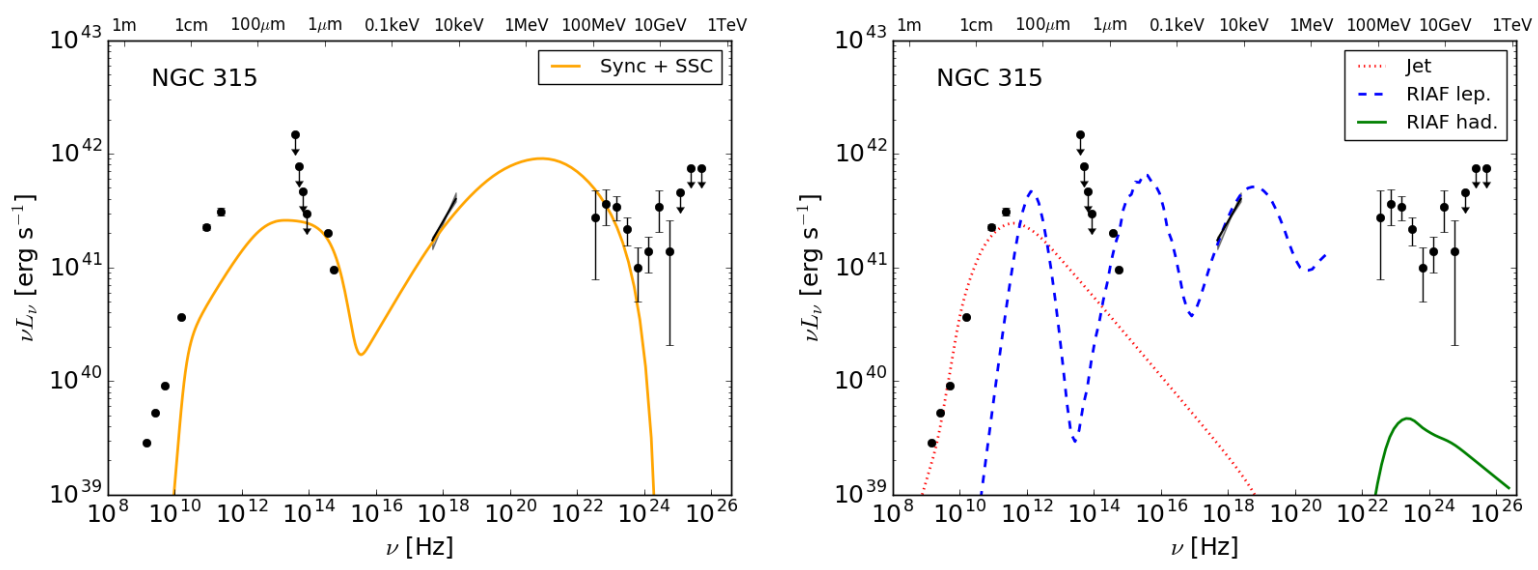

Figure 5.3: Comparison between observed and modelled SEDs for NGC 315. The infrared data were considered as upper limits during the fits, as they are likely contaminated by dust emission. Left: onezone jet model (solid orange lines) where the first and second bumps correspond to the synchrotron and SSC emission components, respectively. Right: RIAF model with thermal lepton (dashed blue) and nonthermal hadronic (green solid) components. The dotted line indicates an illustrative purely synchrotron jet model capable of reproducing the radio observations. The references corresponding to observations taken from other works are listed in Table 5.3.

\begin{tabular}{lccc}
\hline Parameter & Symbol & NGC 315 & NGC 4261 \\
\hline Maximum electron Lorentz factor & $\gamma_{\max }$ & $2.7 \times 10^{4}$ & $2.0 \times 10^{4}$ \\
Break electron Lorentz factor & $\gamma_{\text {brk }}$ & $2.0 \times 10^{3}$ & $3.0 \times 10^{3}$ \\
Lower electron spectral index & $p_{1}$ & 2.0 & 1.8 \\
Doppler factor & $\delta_{D}$ & 1.6 & 1.0 \\
Blob radius [cm] & $R_{b}^{\prime}$ & $1.7 \times 10^{16}$ & $2.1 \times 10^{16}$ \\
Jet power in electrons [erg/s] & $P_{j, e}$ & $3.3 \times 10^{43}$ & $2.7 \times 10^{43}$ \\
Jet power in magnetic field [erg/s] & $P_{j, B}$ & $1.5 \times 10^{41}$ & $1.2 \times 10^{41}$ \\
Comoving magnetic field [G] & $B$ & 0.2 & 0.15 \\
\hline
\end{tabular}

Table 5.4 - SSC model parameters for NGC 315 and NGC 4261. We fixed the following parameters to their typical choices: $\Gamma_{j}=1.5, \gamma_{\min }=1.0$ and $p_{2}=3.0$ (see Section 5.4).

radio emission, but this happens because the observed radio emission likely comes from a different region than that of the rest of the SED.

For the RIAF modeling, the adopted SMBH masses were $7.9 \times 10^{8} M_{\odot}$ for NGC 315 (Woo and Urry, 2002) and $5.2 \times 10^{8} M_{\odot}$ for NGC 4261 (Tremaine et al., 2002). The right panel in Figure 5.3 indicates the best RIAF model found for NGC 315 and the corresponding parameters are listed in Table 5.5. It is known that the compact radio emission in LLAGN is underpredicted by models which only incorporate a thermal distribution of electrons in the RIAF (Yu et al., 2011; Liu and Wu, 2013; Nemmen et al., 2014) and we find the same behavior here for NGC 315 as well as NGC 4261. By including the synchrotron emission of a relativistic jet modeled following Nemmen et al. (2014), the radio emission can be accounted for, as the figure illustrates. The RIAF leptonic emission is able to 


\begin{tabular}{ccc}
\hline Parameter & NGC 315 & NGC 4261 \\
\hline$\dot{M}_{o}$ & $8.9 \times 10^{-3}$ & $3.0 \times 10^{-2}$ \\
$s$ & 0.50 & 0.91 \\
$\delta$ & 0.3 & 0.3 \\
\hline
\end{tabular}

Table 5.5 - Best fit parameters for the RIAF model as applied to NGC 315 and NGC 4261, where $\dot{M}_{o}$ is the accretion rate measured at the outer radius $R_{o}$ of the RIAF in $\dot{M}_{E d d}$ units, $s$ is the index describing the radial variation of the accretion rate due to mass loss in RIAF outflows and $\delta$ is the fraction of energy dissipated via turbulence directly heating electrons. We fixed the parameters $R_{o}=10^{4} R_{S}, \gamma=1.5$, $\alpha=0.3$ and $\beta=0.9$ to their typical choices (see Section 5.4).
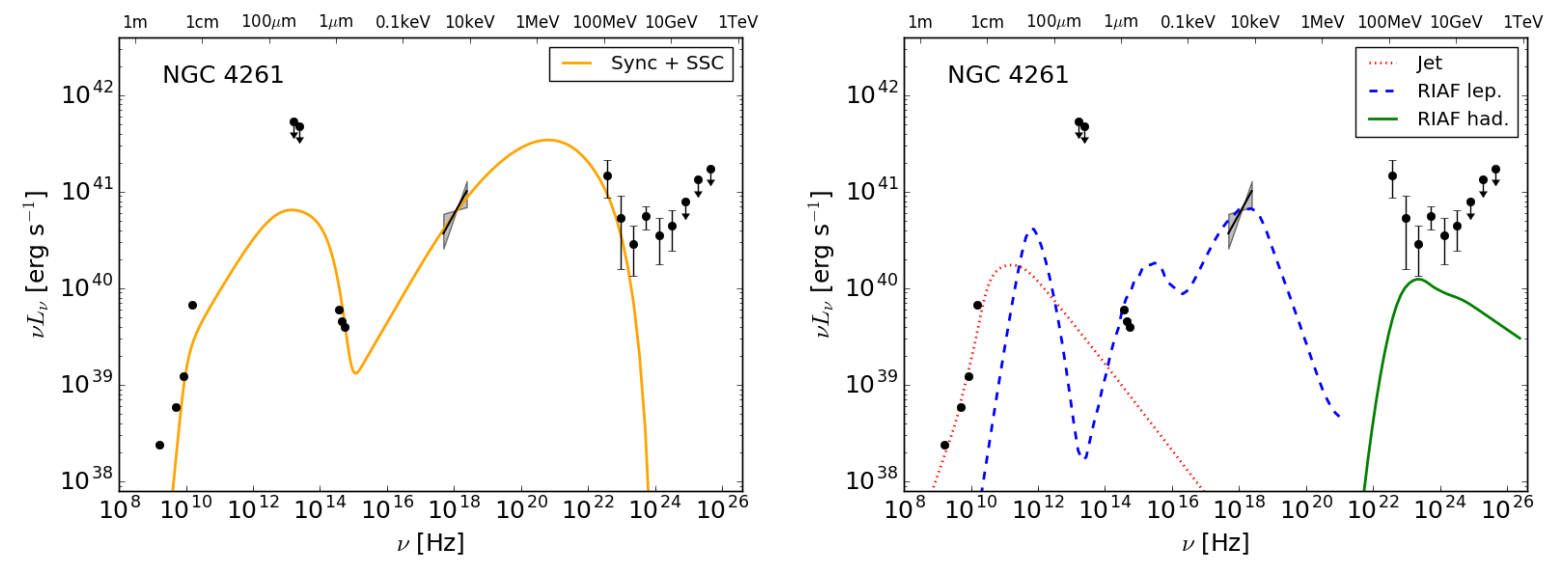

Figure 5.4: Comparison between observed and modelled SEDs for NGC 4261 following the same aesthetic conventions used in Figure 5.3. Left: one-zone synchrotron/SSC jet model. Right: RIAF model. The references corresponding to observations taken from other works are listed in Table 5.3.

broadly account for the $1 \mu \mathrm{m}$ observations and fits the X-ray continuum emission very well. However, the associated hadronic emission fails to reproduce the Fermi-LAT observations, underpredicting the $\gamma$-ray luminosity by about two orders of magnitude.

The results from modeling the SED of NGC 4261 with the jet and RIAF scenarios are displayed in Figure 5.4. Similar to the case of NGC 315, observations at $E<500 \mathrm{MeV}$ are adequately reproduced with an SSC component while the $E>500 \mathrm{MeV}$ data are not. The X-ray spectrum is overpredicted by SSC emission, while the radio spectrum is overall adequately fitted by the jet synchrotron emission.

As before, the RIAF model severely underpredicts the radio emission which can be accounted by synchrotron emission from the misaligned jet. The comptonized RIAF emission accounts for the overall X-ray luminosity even though it does not fit the spectrum in detail. As was found for NGC 315, the RIAF hadronic emission fails to reproduce the Fermi-LAT observations, underpredicting the $\gamma$-ray luminosity by a factor of four.

To probe the size of their $\gamma$-ray emitting regions, we generated $\gamma$-ray photon flux light 
curves with 6 and 3 month bins for both NGC 315 and 4261, starting on August 2008 up to November 2018. The lack of significant variability, however, did not allow for any precise conclusion. These results are in agreement with Abdo et al. (2010) and Grandi et al. (2013), where they found that FRI galaxies are generally characterized by quiescent $\gamma$-ray light curves. Such behavior can be due to the presence of a more structured jet in FRIs, with most of the $\gamma$-ray emission being produced in less beamed more extended regions Grandi et al. (2013), like the extended emission observed for Centaurus A (Fermi LAT Collaboration, 2010b).

\subsection{Discussion}

The four $\gamma$-ray bright LLAGN in the Palomar sample consist of misaligned radio galaxies, each displaying resolved, kpc-scale radio jets (Jones and Wehrle, 1997; Junor et al., 1999; Cotton et al., 1999; Walker et al., 2000). A comparison of models to the SEDs of these sources indicate that they are well described by a jet-dominated model in the form of a one-zone synchrotron/SSC jet scenario (cf. previous section), similarly to blazars (Finke, 2013; Ghisellini and Tavecchio, 2015). It was also found that models invoking the origin of the electromagnetic radiation in a RIAF failed to explain the Fermi-LAT observations while being able to reproduce the radio-to-X-ray emission. Since the $\gamma$-ray-bright LLAGN consist of powerful radio galaxies, it is not entirely unexpected that their emission can be dominated by a jet even if misaligned to the observer's line of sight.

Unfortunately, the other 193 LLAGN were not detected by Fermi-LAT $($ TS $<25$ ) and provided only upper limits (Appendix 5.8). Two of these sources, namely NGC 4374 (M84) and NGC 4151, present a shy $\gamma$-ray emission above the $3 \sigma$ level (TS $>9$ ) and may contribute to the correlation seen in Figure 5.1 in the next few years, when more FermiLAT data will be available. Despite the small number of detections, it is still possible to use the hint of correlation between the luminosities in the $\gamma$-ray band with the radio and X-ray ones and make educated guesses for the production site of high-energy photons in the sample. A weak radio- $\gamma$-ray correlation was found in the sample where only the radiobrightest LLAGN were actually detected in $\gamma$-rays. On the other hand, no X-ray $/ \gamma$-ray correlation was found, with most X-ray bright sources not being observed in $\gamma$-rays. These results are interpreted as pointing to a different origin for the $\gamma$-ray and radio radiation 
compared to the X-rays. The $\gamma$-rays would be produced in the jet which also produce the seed radio photons which are upscattered to high energies, disfavoring models where the $\gamma$-ray emission is coming from hadronic interactions in the RIAF.

The lack of correlation between $\gamma$-rays and X-rays (Figure 5.2) can be interpreted as the X-ray photons being mostly produced in the RIAF when the LLAGN presents no $\gamma$-ray emission. In summary, this suggests that different particle populations are responsible for the different parts of the spectrum in LLAGN as opposed to models that invoke the same particle populations as being responsible for the entirety of the electromagnetic broadband emission (Markoff et al., 2005; Khiali et al., 2015). On the other hand, the X-rays directly scales with the $\gamma$-rays for the four $\gamma$-ray-bright AGN (Figure 5.2), indicating that for these few AGN with very powerful jets, the jets significantly contribute to the X-ray emission. We stress that these four AGN are well known by presenting jets or even bubbles resolved in X-rays (Young et al., 2002; Gonzalez-Martin et al., 2006; Worrall et al., 2010; Fabian et al., 2015).

These results are not easily accommodated in the context of models which predict the relative spectral dominance of the RIAF versus the jet, such as the one proposed by Yuan and Cui (2005). According to Yuan and Cui (2005), the X-ray emission of the system should be dominated by the jet rather than by the RIAF when $L_{X}<L_{\mathrm{X} \text {,crit }}$, where $L_{\mathrm{X} \text {,crit }}(M) \propto$ $M^{-0.17}$ is a critical value according to which $L_{\mathrm{X}, \text { crit }}\left(10^{8} M_{\odot}\right)=2.4 \times 10^{39} \mathrm{erg} \mathrm{s}^{-1}$ and $L_{\mathrm{X}, \text { crit }}\left(10^{9} M_{\odot}\right)=1.6 \times 10^{40} \mathrm{erg} \mathrm{s}^{-1}$. In the present sample, all four $\gamma$-ray-bright LLAGN have $L_{X}>L_{\mathrm{X} \text {,crit }}$ but are well-explained by jet-dominated models. Given the absence of a $L_{\gamma}-L_{\mathrm{X}}$ correlation, for most of the sample the X-rays should not be coming from the jet which is in conflict with Yuan \& Cui's prediction since most LLAGN are typified by $L_{X}<L_{\mathrm{X}, \text { crit }}$.

The resulting parameters for the jet models as applied to NGC 315 and NGC 4261 are typical of other low-power radio galaxies (Abdo et al., 2009; Tanada et al., 2018), with lower Doppler factors compared to BL Lacs (Costamante et al., 2018). Such low-power radio galaxies are on the threshold of detection with Fermi-LAT. Future observations of the Palomar sample with higher-sensitivity $\gamma$-ray observatories such as the Cherenkov Telescope Array (CTA; Hassan et al., 2017) and the All-sky Medium Energy Gamma-ray Observatory (AMEGO; McEnery et al., 2019) should produce more detections of LLAGN. AMEGO is particularly interesting for this purpose as our analysis (Figures 5.3 and 5.4) 
indicate that the peak output energy is in the soft $\gamma$-ray region of the SEDs. Indeed, LLAGN like NGC 4374 (M84) and NGC 4151 may be the best candidates for future observations, given their computed TS values of 15 and 11, respectively (see Appendix $5.8)$.

The highest energy observations in Figures 5.3 and 5.4 do not agree well with the SSC emission from the jet model. This suggests that a one-zone synchrotron/SSC interpretation for the whole SEDs of NGC 315 and NGC 4261 does not provide a complete explanation for the SEDs. To test for a possible hardening of the spectra starting at few $\mathrm{GeV}$, similar to what is observed for Centaurus A (Abdalla et al., 2018), two more models were fitted to the $\gamma$-ray data: a Broken Power-law and a Log Parabola with a concave upwards shape. Both models, however, are less significant than a simple power-law model, suggesting that the hardening is not statistically significant for the $\gamma$-ray spectra.

Finally, if the weak $\gamma$-ray-radio correlation holds, it disfavours models where the $\gamma$-ray emission is generated by particles accelerated in a magnetospheric gap in the surroundings of the SMBH (Levinson and Rieger, 2011; Rieger, 2011; Hirotani, 2018; Rani, 2019). In this magnetospheric gap scenario, the $\gamma$-ray luminosity increases with decreasing accretion rate, and thus an anti-correlation between the magnetospheric gap $\gamma$-ray emission and RIAF radio emission is predicted (Hirotani, 2018). On the other hand, the high-energy emission originating in the jet is expected to naturally correlate with the jet radio synchrotron emission (Max-Moerbeck et al., 2014; Ramakrishnan et al., 2015). Our data thus point to a jet origin for the observed radio and $\gamma$-ray emissions, although we stress that the observed $\gamma$-ray-radio correlation shown in Figure 5.1 is relatively weak.

\subsection{Conclusions}

This is the first systematic study of the emission properties of supermassive black holes accreting at low rates in the $\gamma$-ray energy band, where we have analyzed 10.25 years of Fermi-LAT observations of LLAGN in the Palomar sample. Our main conclusions can be summarized as follows:

(i) Of the 197 LLAGN in the Palomar sample, only four sources were detected in $\gamma$-rays with significance above $5 \sigma$ (TS > 25). These four sources correspond to misaligned FRI radio galaxies, each displaying kpc-scale radio jets: NGC 315, NGC 1275, NGC 4261 and 
NGC 4486 (M87).

(ii) The presence of radio jets in LLAGN seems to be a necessary condition for observing a significant $\gamma$-ray counterpart: the four $\gamma$-ray-bright sources are also the radio-brightest in the sample and we see a possible hint of correlation between $\gamma$-ray and radio luminosities, which disfavours RIAF and magnetospheric gap models for $\gamma$-ray emission.

(iii) The presence of strong X-ray emission does not necessarily imply high $\gamma$-ray luminosity: only one of the $\gamma$-ray-bright sources is among the seven X-ray-brightest and we find no correlation between $L_{X}$ and $L_{\gamma}$.

(iv) We interpret the above results as indicating that in most LLAGN the $\gamma$-ray and radio emission are produced in the relativistic jet while the X-rays are coming from the accretion flow.

(v) We performed a detailed comparison of RIAF and jet models to the SEDs of NGC 315 and NGC 4261. We have found that they are well-described by a jet-dominated model in the form of a one-zone synchrotron/SSC leptonic scenario, similar to blazars and other FRI radio galaxies. The hadronic emission from the RIAF failed to explain the Fermi-LAT observations.

(vi) The jet model is unable to reproduce the spectrum at energies above $1 \mathrm{GeV}$, suggesting that a more complex, multi-component model may be more appropriate to account for the high-energy tail of Fermi-LAT observations. A more detailed analysis of the observations indicate that the hardening at $>1 \mathrm{GeV}$ is not significant.

Future observational campaigns focused on LLAGN with CTA and AMEGO should produce more $\gamma$-ray detections of sub-Eddington active SMBHs.

\section{Acknowledgements}

This work was supported by FAPESP (Fundação de Amparo à Pesquisa do Estado de São Paulo) under grants 2016/25484-9, 2018/24801-6 (R.M.), 2017/01461-2 (R.N.), 2016/24857-6 and 2019/10054-7 (I.A.). J.D.F. was supported by NASA under contract S-15633Y. We thank Simone Garrappa, Vaidehi Paliya, Fabio Cafardo and the anonymous referee for the constructive comments allowing us to improve the manuscript. The full acknowledgments can be found in the original paper by de Menezes et al. (2020). 


\subsection{Appendix: Energy flux upper limits for all AGN non-detected in gamma-rays}

Energy flux upper limits with $95 \%$ confidence levels and integrated over the whole analysis energy range are provided in Table 5.6, where the sources have been assumed to have a power-law spectrum with a spectral index fixed in 2. The TS values are also listed and highlight the shy presence of NGC 4374 (M84) and NGC 4151, known to host remarkably powerful radio cores (Ly et al., 2004; Mundell et al., 2003), as possible $\gamma$-ray emitters (significance $\sim 3 \sigma$ ).

\begin{tabular}{l|c|c} 
Source & $\begin{array}{c}\text { Upper limit } \\
10^{-13} \mathrm{erg} \mathrm{cm}^{-2} \mathrm{~s}^{-1}\end{array}$ & TS \\
\hline IC239 & 5.09 & 0.04 \\
IC356 & 1.89 & 0.00 \\
IC520 & 3.06 & 0.00 \\
IC1727 & 5.32 & 0.00 \\
NGC185 & 26.92 & 3.31 \\
NGC266 & 5.70 & 0.00 \\
NGC404 & 6.07 & 0.18 \\
NGC410 & 6.22 & 0.17 \\
... & $\ldots$ & $\ldots$
\end{tabular}

Table 5.6 - Energy flux upper limits and TS values for all 193 AGN non-detected in $\gamma$-rays. The energy range adopted is from $100 \mathrm{MeV}$ up to $300 \mathrm{GeV}$. The full version of this table can be found in de Menezes et al. (2020). 
Chapter 6

\section{The infrared colors of gamma-ray blazars}

This thesis chapter is a shortened version of the paper "Optical characterization of WISE selected blazar candidates" by de Menezes et al. (2019), published in the journal Astronomy \& Astrophysics, volume 630, A55. The author was responsible for writing the paper, analyzing the data, and finding the main results. The original work can be found online here.

\section{Abstract}

Context. Over the last decade more than five thousand $\gamma$-ray sources have been detected by the Large Area Telescope (LAT) on board Fermi Gamma-ray Space Telescope. Given the positional uncertainty of the telescope, nearly $30 \%$ of these sources remain without an obvious counterpart in lower energies. This motivated the release of new catalogs of $\gamma$-ray counterpart candidates and several follow up campaigns in the last decade.

Aims. Recently, two new catalogs of blazar candidates were released, they are the improved and expanded version of the Wide-field Infrared Survey Explorer (WISE) Blazar-Like Radio-Loud Sources (WIBRaLS2) catalog and the Kernel Density Estimation selected candidate BL Lacs (KDEBLLACS) catalog, both selecting blazar-like sources based on their infrared colors from WISE. In this work we characterize these two catalogs, clarifying the true nature of their sources based on their optical spectra from SDSS data release 15, thus testing how efficient they are in selecting true blazars.

Methods. We first selected all WIBRaLS2 and KDEBLLACS sources with available optical spectra in the footprint of Sloan Digital Sky Survey data release 15. Then we analyzed these spectra to verify the nature of each selected candidate and see which fraction of the ca- 
talogs is composed by spectroscopically confirmed blazars. Finally, we evaluated the impact of selection effects, specially those related to optical colors of WIBRaLS2/KDEBLLACS sources and their optical magnitude distributions.

Results. We found that at least $\sim 30 \%$ of each catalog is composed by confirmed blazars, with quasars being the major contaminants in the case of WIBRaLS2 $(\approx 58 \%)$ and normal galaxies in the case of KDEBLLACS $(\approx 38.2 \%)$. The spectral analysis also allowed us to identify the nature of 11 blazar candidates of uncertain type (BCUs) from the Fermi-LAT 4th Point Source Catalog (4FGL) and to find 25 new BL Lac objects.

\subsection{Introduction}

One of the main challenges of modern $\gamma$-ray astronomy in the era of Fermi Large Area Telescope (LAT) is the association of $\gamma$-ray sources with their low-energy counterparts (Massaro et al., 2015, 2016). The main problem underlying it is the large positional uncertainty of $\gamma$-ray detected sources, typically $\sim 4^{\prime}$ in the Fermi-LAT 4 th Point Source Catalog ${ }^{1}$ (4FGL; Abdollahi et al., 2020). The association task also affects source classification that covers up to $\sim 70 \%$ of the 4 FGL leaving 1521 unidentified/unassociated $\gamma$-ray sources (UGSs) to date.

These UGSs are distributed uniformly throughout the whole sky - although showing some concentration towards the Galactic plane $\left(|b|<20^{\circ}\right)$ - indicating that most of them could have extragalactic nature. The $\gamma$-ray sky is mainly populated by non-thermal sources and in particular $\sim 80 \%$ of 4FGL associated sources are classified as blazars. These blazars belong to one of the rarest class of active galactic nuclei (AGNs), whose emission arises from particles accelerated in a relativistic jet closely aligned with line of sight (Blandford et al., 1978). It is therefore expected that a significant fraction of UGSs is composed of blazars, at least at high Galactic latitudes.

Blazars are divided into BL Lacs (BZBs) and blazars of quasar type, labelled in the Roma-BZCAT as BZQs (Massaro et al., 2015), and are classified based on their optical spectra, where the former have featureless optical spectra, or only absorption lines of Galactic origin and weak and narrow ( $<5 \AA$; Stickel et al., 1991; Landoni et al., 2014, 2015,b) emission lines; and the latter have flat radio spectra, with optical spectra showing

\footnotetext{
${ }^{1}$ https://fermi.gsfc.nasa.gov/ssc/data/access/lat/8yr_catalog/
} 
broad emission lines and a dominant intrinsically blue continuum. Blazars, emitting nonthermal radiation over the whole electromagnetic spectrum, also show large variability at all wavelengths, a flat or inverted radio spectrum, significant polarized emission and in some cases even apparent super-luminal motion (Urry and Padovani, 1995; Abdo et al., 2010).

Blazars occupy a specific region in the mid-infrared (IR) color space defined by the Wide-field Infrared Survey Explorer filters (WISE; Wright et al., 2010). This region is known as the WISE Blazar Strip (Massaro et al., 2011b, 2012; D'Abrusco et al., 2012) when it is described in a two-dimensional space, and locus when it is modeled in the full three-dimensional WISE color space (D'Abrusco et al., 2014). Such distinctive mid-IR colors are due to the non-thermal emission arising from their relativistic jets (Böttcher, 2007). This discovery led to search for blazar-like sources lying within the positional uncertainty ellipses of UGSs that could be their potential counterparts (D'Abrusco et al., 2014; Massaro et al., 2015).

One of the catalogs that provides the largest number of associated blazar candidates for Fermi-LAT catalogs is the WISE Blazar-Like Radio-Loud Sources catalog (WIBRaLS; D'Abrusco et al., 2014, 2019). Selected sources in the WIBRaLS are detected in all four mid-IR WISE bands (nominally at 3.4, 4.6, 12 and $22 \mu \mathrm{m}$ ) and show mid-IR colors similar to confirmed Fermi blazars. Sources in WIBRaLS are also required to have a radio-loud counterpart (see more details in Section 6.2.1). In the latest release of the WIBRaLS catalog (hereinafter WIBRaLS2), an additional list of candidate BZBs has been built based on a kernel density estimation (KDE) technique, namely KDEBLLACS (D'Abrusco et al., 2019). Sources in KDEBLLACS are also radio-loud. Sources in both catalogs are selected with Galactic latitude $|b|>10^{\circ}$.

The main goal of the present analysis is to characterize sources listed in both WIBRaLS2 and KDEBLLACS, on the basis of the optical spectra available for those lying in the footprint of the Sloan Digital Sky Survey (SDSS; York et al., 2000) 15th data release (DR15; Aguado et al., 2019). We aim to classify them spectroscopically mainly to determine the source classes that contaminate the WIBRaLS2 selection criteria. SDSS is ideal to achieve our purposes since it is the survey with higher number of spectroscopic objects available in the literature.

The paper is organized as follows: in Section 6.2 we describe samples used to carry out 
our analysis, providing basic details of both WIBRaLS2 and KDEBLLACS, selecting those sources in the SDSS footprint. Sections 6.3 and 6.4 describe our spectral classification method and results, respectively. Finally, we summarize our results and conclusions in Section 6.5.

Throughout this work we use $\mathrm{h}=0.70, \Omega_{m}=0.30$, and $\Omega_{\Lambda}=0.70$, where the Hubble constant is $H_{0}=100 \mathrm{~h} \mathrm{~km} \mathrm{~s}^{-1} \mathrm{Mpc}^{-1}$ (Tegmark et al., 2004). Spectral indices are defined by flux density $S_{\nu} \propto \nu^{-\alpha}$, indicating a flat spectrum when $\alpha<0.5$. The WISE magnitudes are in the Vega system and are not corrected for the Galactic extinction, since, as shown in D'Abrusco et al. (2014), such correction only affects the magnitude at $3.4 \mu \mathrm{m}$ for sources lying close to the Galactic plane and it ranges between $2 \%$ and $5 \%$ of the magnitude, thus not affecting significantly the results. WISE bands are indicated as W1, W1, W3 and W4, and correspond respectively to the nominal wavelengths at 3.4, 4.6, 12, and $22 \mu \mathrm{m}$.

\subsection{Sample selection}

For this analysis we used observations available in the AllWISE catalog (Cutri et al., 2013 , 2014), which contains astrometry and photometry in the IR for $\sim 3 \times 10^{7}$ sources in W1, W2, W3 ad W4 bands, it includes data from the cryogenic and post-cryogenic survey phases (Mainzer et al., 2011), increasing to $\sim 10^{8}$ sources detected only on the first three bands W1, W2 and W3.

\subsubsection{WIBRaLS2}

The WIBRaLS2 catalog was conceived to provide a good sample of potential $\gamma$-ray sources based on IR and radio data. It includes mid-IR sources detected in the four WISE filters, with colors similar to those of blazars listed in the Fermi-LAT 4-year Point Source Catalog (3FGL; Acero et al., 2015), spatially cross-matched with a radio counterpart found in one of the three major radio surveys: the National Radio Astronomy Observatories Very Large Array (VLA) Sky Survey (NVSS; Condon et al., 1998), the VLA Faint Images of the Radio Sky at Twenty-cm Survey (FIRST; White et al., 1997; Helfand et al., 2015) and the Sydney University Molonglo Sky Survey Source Catalog (SUMSS; Mauch et al., 2003); and selected to be radio-loud based on their $q_{22}$ spectral parameter, defined as $q_{22}=\log \left(S_{22 \mu m} / S_{\text {radio }}\right)$, with $S_{22 \mu m}$ and $S_{\text {radio }}$ being the flux densities at $22 \mu m$ and in 


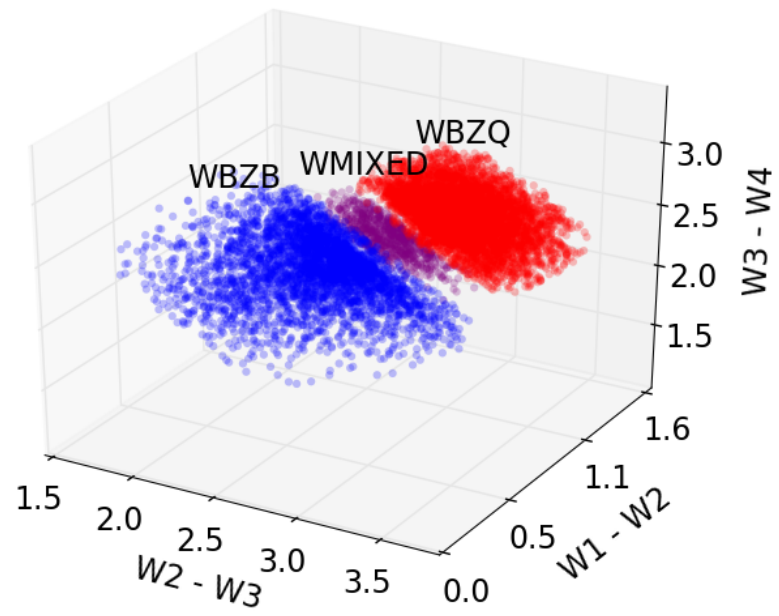

Figure 6.1: The whole WIBRaLS2 sample divided into three categories accordingly to the sources' IR colors: BZB candidates (WBZBs, in blue), BZQ candidates (WBZQs, in red) and blazars with intermediate colors (WMIXEDs, in purple).

radio respectively. The total number of blazar candidates in WIBRaLS2 is 9541 and they are classified (see Figure 6.1) as candidate BZBs, candidate BZQs or MIXEDs (hereinafter WBZBs, WBZQs and WMIXEDs, respectively), the latter defined as those with midIR colors which are intermediate between WBZBs and WBZQs (for more details, see D’Abrusco et al., 2019).

In this paper, we have cross-matched the WIBRaLS2 catalog with SDSS DR15 associating blazar candidates listed therein with their optical counterparts within an angular separation of $2^{\prime \prime}$ (Massaro et al., 2014). We found a total number of 3407 unique associations and then used the SDSS Science Archive Server ${ }^{2}$ to select only those sources with an available optical spectrum. We then selected only those sources with a spectral signal to noise ratio $(\mathrm{S} / \mathrm{R})$ above 5 and sources with $\mathrm{S} / \mathrm{R}<5$ but listed in Roma-BZCAT. This led us to a final sample of 1424 WIBRaLS2 sources, which corresponds to $\sim 15 \%$ of the whole WIBRaLS2. A flowchart describing the effects of our selection of the number of candidates in the WIBRaLS2 catalog is displayed in Figure 6.2.

\subsubsection{KDEBLLACS}

The KDEBLLACS catalog was built by first applying a kernel density estimation (KDE) to a two-dimensional distribution of training set sources of BZB type in the WISE W1-W2 $\times$ W2-W3 color diagram to determine its probability distribution function (PDF). Sources were then selected when located within the $90 \%$ isodensity contour of the training set

\footnotetext{
2 https://dr15.sdss.org/optical/spectrum/search
} 


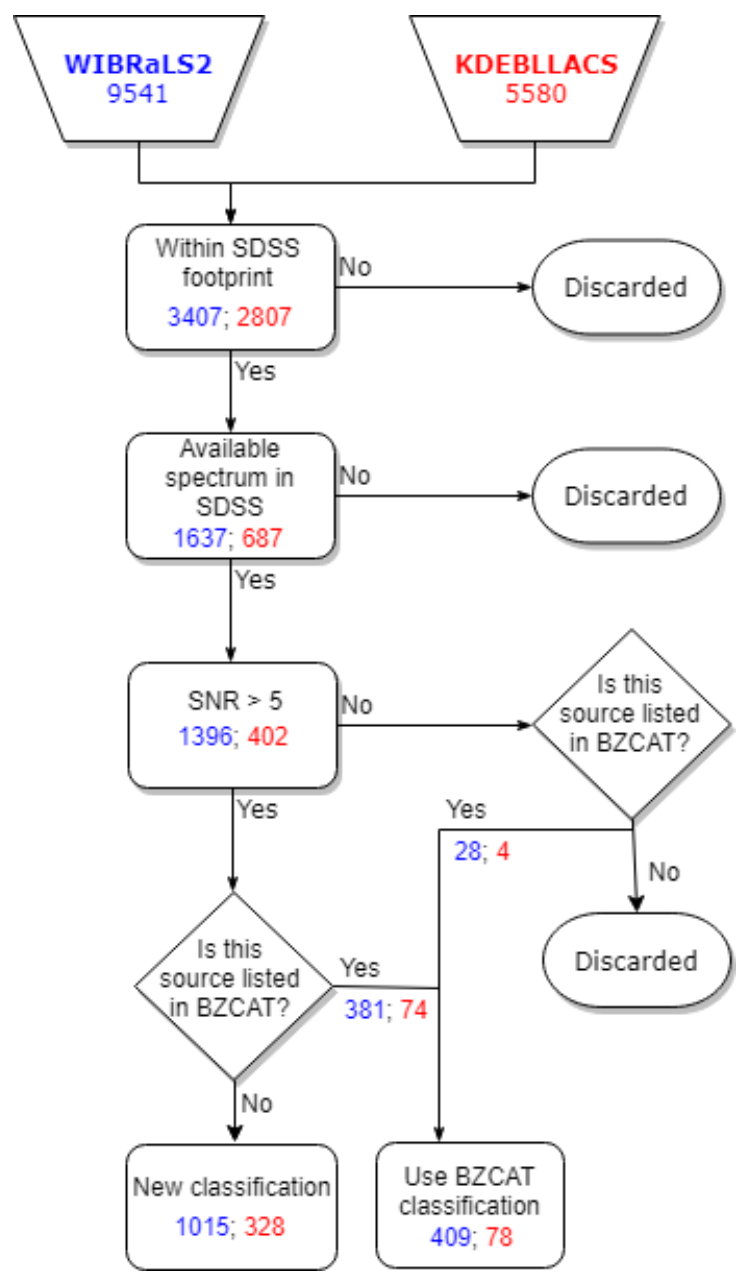

Figure 6.2: Flow chart with all steps adopted in the sample selection and spectral classification. The numbers in blue refer to sources in WIBRaLS2 while numbers in red refer to sources in KDEBLLACS after each step.

(D'Abrusco et al., 2019) built with the KDE. The color uncertainty ellipses of each source in KDEBLLACS must be fully contained in this $90 \%$ contour. Sources in KDEBLLACS are radio-loud according to the $q_{12}$ parameter, defined as $q_{12}=\log \left(S_{12 \mu m} / S_{\text {radio }}\right)$, with $S_{12 \mu m}$ and $S_{\text {radio }}$ being the flux densities at $12 \mu m$ and in radio respectively (D'Abrusco et al., 2019).

KDEBLLACS lists 5580 candidate BZBs (hereinafter labelled as KBZBs). This number decreases to 2807 sources lying in the SDSS footprint, however, only 402 of them have good (i.e., S/R > 5) optical spectra. Our final sample consists of these 402 sources together with 4 sources listed in Roma-BZCAT which have low significance $(\mathrm{S} / \mathrm{R}<5)$ SDSS spectra. This sample corresponds to $7 \%$ of the whole KDEBLLACS (see Figure 6.2 for details). 


\subsection{Spectral analysis and classification}

To classify sources in WIBRaLS2 and KDEBLLACS according to their optical spectra, we started by cross-matching both samples, previously defined, with Roma-BZCAT (Massaro et al., 2015), finding a total of 409 counterparts (out of 1424) for WIBRaLS2 and 78 (out of 404) for KDEBLLACS, respectively. These sources were already classified as blazars and we assumed their classification without further check.

We then adopted the following classification scheme, divided into sources listed in Roma-BZCAT and remaining sources:

- Roma-BZCAT

- BZB

- BL Lac - galaxy-dominated (BZG)

- Blazar uncertain type (BZU)

- BZQ

- Remaining sources

$-\mathrm{BZB}$

- Quasi-stellar object (QSO), or BZQ in some cases

- Low-luminosity active galactic nucleus (LLAGN)

- Star-forming galaxy

- BZG

- Galaxy

- Star

Figure 6.3 shows the typical smoothed spectra for all classes discussed above. The criteria adopted in the classification of the new analyzed sources is explained below.

Particularly for blazars, we classified sources as BZBs when they showed a featureless optical spectra with a dominant blue continuum, and as QSOs when presenting redshifted broad emission lines again above a dominant blue continuum coupled with a radio luminosity $L_{r}$ greater than $10^{38} \mathrm{erg} \mathrm{s}^{-1}$. 

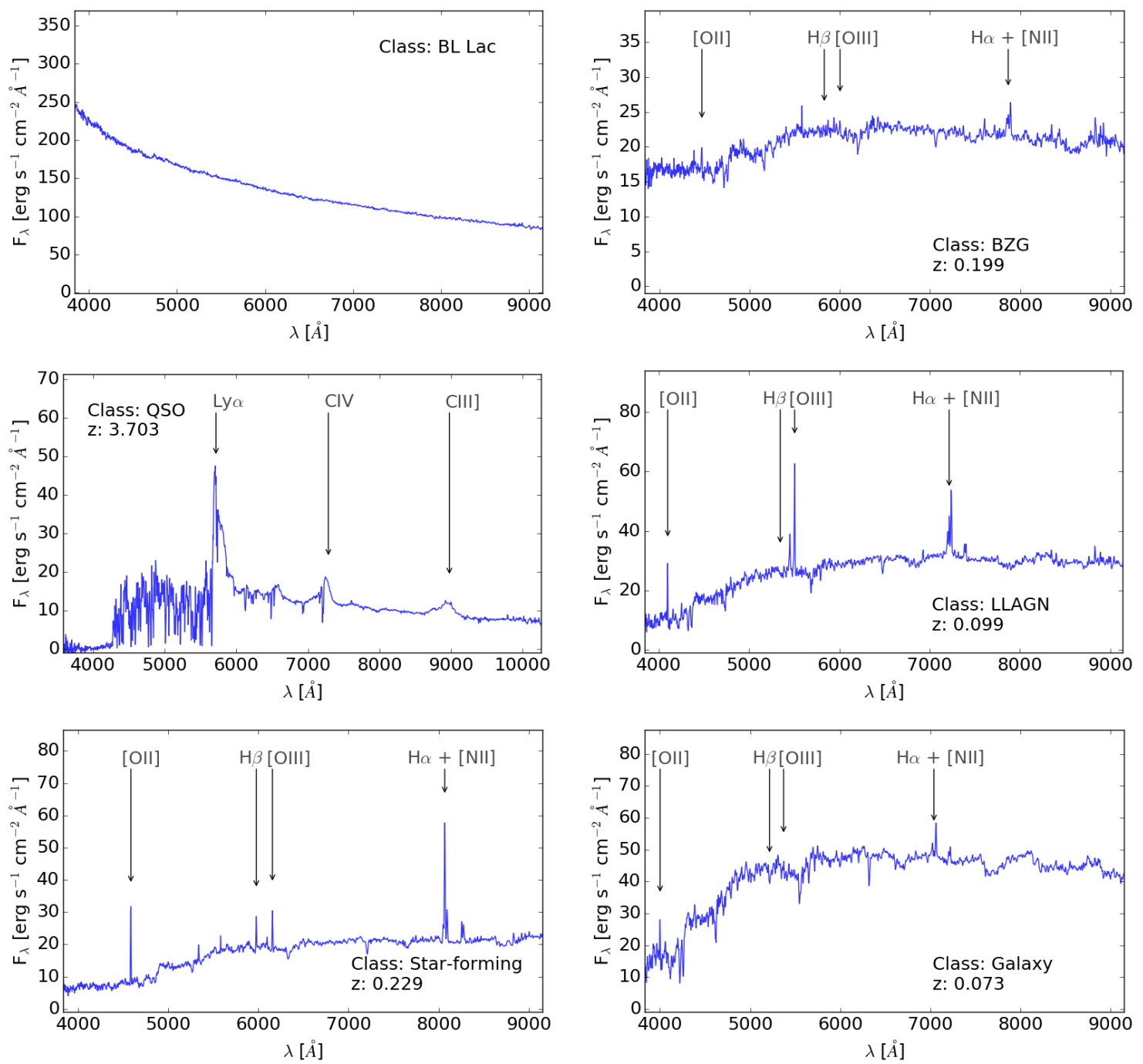

Figure 6.3: Typical smoothed spectra in observed wavelengths for each one of the spectral categories found in WIBRaLS2 and KDEBLLACS.

On the other hand, LLAGNs and star-forming galaxies were distinguished based on their line ratios $[\mathrm{OIII}] / \mathrm{H}_{\beta}$ and $[\mathrm{NII}] / \mathrm{H}_{\alpha}$ according to the BPT diagram (Baldwin et al., 1981; Kewley et al., 2006). A total of 4 star-forming galaxies have been identified in WIBRaLS2 and only 2 in KDEBLLACS.

To distinguish between normal galaxies and BZGs, we measured their relative flux depression bluewards the Ca II break. This parameter was defined as $C B=\left(F_{+}-F_{-}\right) / F_{+}$, with $F_{+}$and $F_{-}$meaning the flux densities measured in ranges of $200 \AA$ at wavelengths just above and below that of the Ca II H\&K break (Dressler and Shectman, 1987; Massaro et al., 2012). As in Stocke et al. (1991), we adopted a threshold value of 0.25 for CB to ensure the presence of a substantial non-thermal continuum (i.e., $\mathrm{CB}<0.25$ ) leading to a BZG classification; otherwise, it was classified as a normal galaxy. Finally, a total of 3 
and 2 stars were found contaminating WIBRaLS2 and KDEBLLACS respectively; four of them typical cold M- or G-type, and one white dwarf.

\subsection{Results}

The optical classification previously described enabled us to characterize WIBRaLS2 and KDEBLLACS subsamples. The results are presented here split in the following subsections.

\subsubsection{WIBRALS2}

We analyzed a total of 1424 spectra (1396 with $S / N>5$ and 28 with $S / N<5$ but with a counterpart in Roma-BZCAT) among the 9541 sources available in WIBRaLS2 but only 3407 in the SDSS footprint. According to their WIBRaLS2 classification, these sources have been divided into 471 WBZBs, 833 WBZQs and 120 WMIXEDs. As can be seen in Figure 6.4, their main contaminant class is QSOs. A significant fraction of these QSOs, however, may have a radio flat spectrum, indicating that they could be indeed blazars of BZQ type. Subsequently, we found that 17.6\% of WBZBs show a featureless optical spectrum; $26.1 \%$ of WBZQs are confirmed BZQs (i.e., radio flat spectrum) and $30.8 \%$ of WMIXEDs sources are also BZQs.
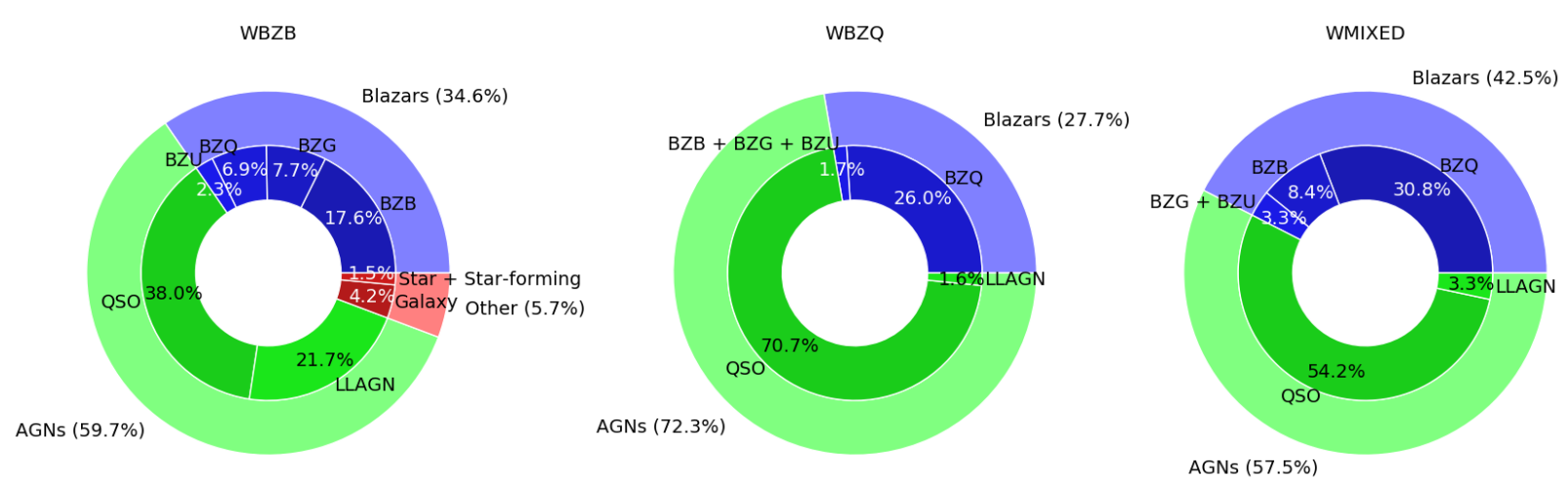

Figure 6.4: Nested pie charts for the 3 classes of sources described in WIBRaLS2 (WBZB, WBZQ and WMIXED). The major contaminant in all categories are AGNs. A significant fraction of these AGNs, however, may be blazars (BZQ). The subclass LLAGN is represented by Seyferts and LINERs.

As can be seen in the left and middle panels of Figure 6.5, the majority of contaminants in WIBRaLS2 are concentrated in the WBZQ and WMIXED regions of the mid-IR colorcolor diagram. On the other hand, the WBZB sample is cleaner, and its purity can reach 
$\sim 50 \%$ in the area bounded by the $68 \%$ isodensity contours of 4 FGL $\gamma$-ray blazars (Figure 6.5 , right panel).
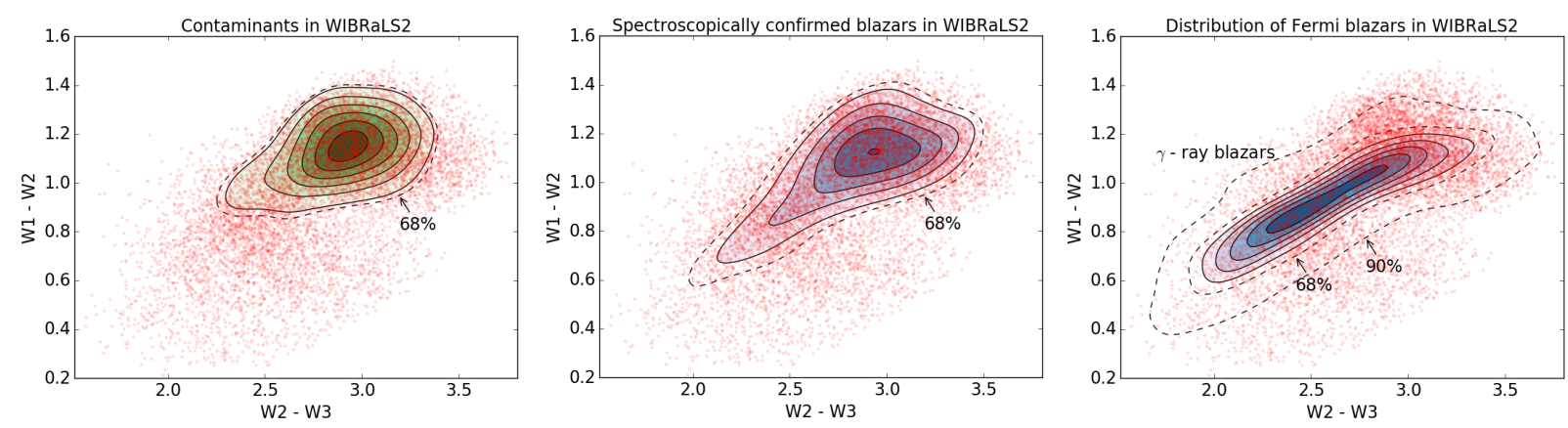

Figure 6.5: Contaminants in WIBRaLS2. Left: Contamination of WIBRaLS2 obtained based on a KDE and using the SDSS DR15 spectra of 953 sources (833 QSOs, 100 Seyferts and 20 LINERs) as training sample. The contaminants are mainly QSOs concentrated in the WBZQ region of the color-color diagram (as pointed in Figure 6.1). Middle: PDF of spectroscopically confirmed blazars in WIBRaLS2 (KDE training set composed of $281 \mathrm{BZQs}, 95 \mathrm{BZBs}, 36$ BZGs and $24 \mathrm{BZUs}$ ). Most of them lie in the most contaminated region of WIBRaLS2. Right: PDF of $\gamma$-ray blazars obtained using 1320 cross-matches between 4FGL blazars and WIBRaLS2 sources as a training sample for a KDE. The $\gamma$-ray blazars are mainly located in a narrow strip peaking in the WBZB region. The solid lines represent the isodensity contours obtained with the KDE, while the dashed lines indicate the $68 \%$ and (for the last panel) $90 \%$.

\subsubsection{KDEBLLACS}

With a total of 406 spectra analyzed, KBZBs in KDEBLLACS were classified as follows: 75 BZBs (60 of them confirmed in Roma-BZCAT), 39 BZGs (17 confirmed in RomaBZCAT), 155 normal galaxies, 47 LLAGNs (20 LINERs and 27 Seyferts), 84 QSOs, 2 confirmed BZQs (1 of them listed in Roma-BZCAT), 2 stars and 2 star-forming galaxies, all according to the criteria previously described. Figure 6.6 summarizes these results in a nested pie chart.

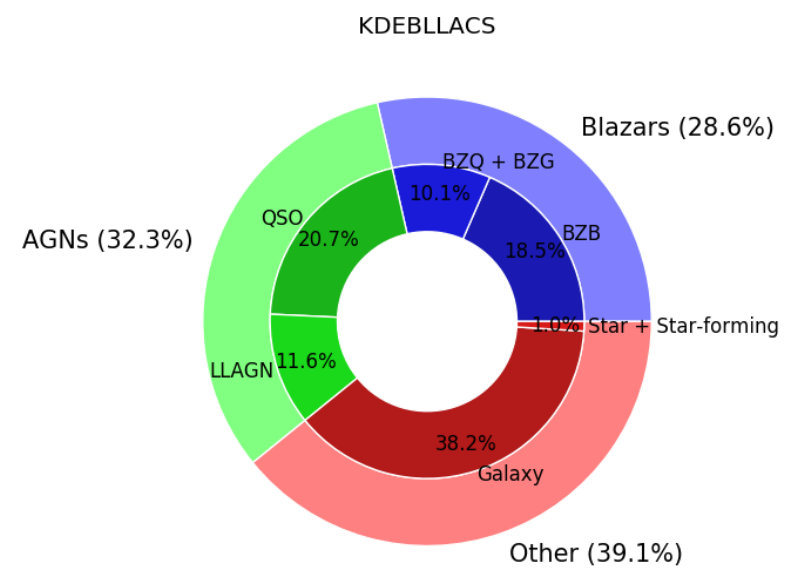

Figure 6.6: Contaminants for KDEBLLACS divided by classes. The major contaminant for this catalog are normal galaxies. Some fraction of the QSOs can have a flat radio spectrum and be BZQs. 
It is worth noting that normal galaxies and QSOs are the major contaminant classes of KDEBLLACS selection criteria. After applying two KDEs to this sample, one using spectroscopically confirmed QSOs and the other using spectroscopically confirmed galaxies as training sets, we observe (as expected) that these contaminants are concentrated towards the edges of the mid-IR color-color diagram (Figure 6.7, left panel), mainly in the bottom and upper-right corners. Indeed, most of the BZBs classified in 4FGL lie outside of these regions (Figure 6.7, right panel).
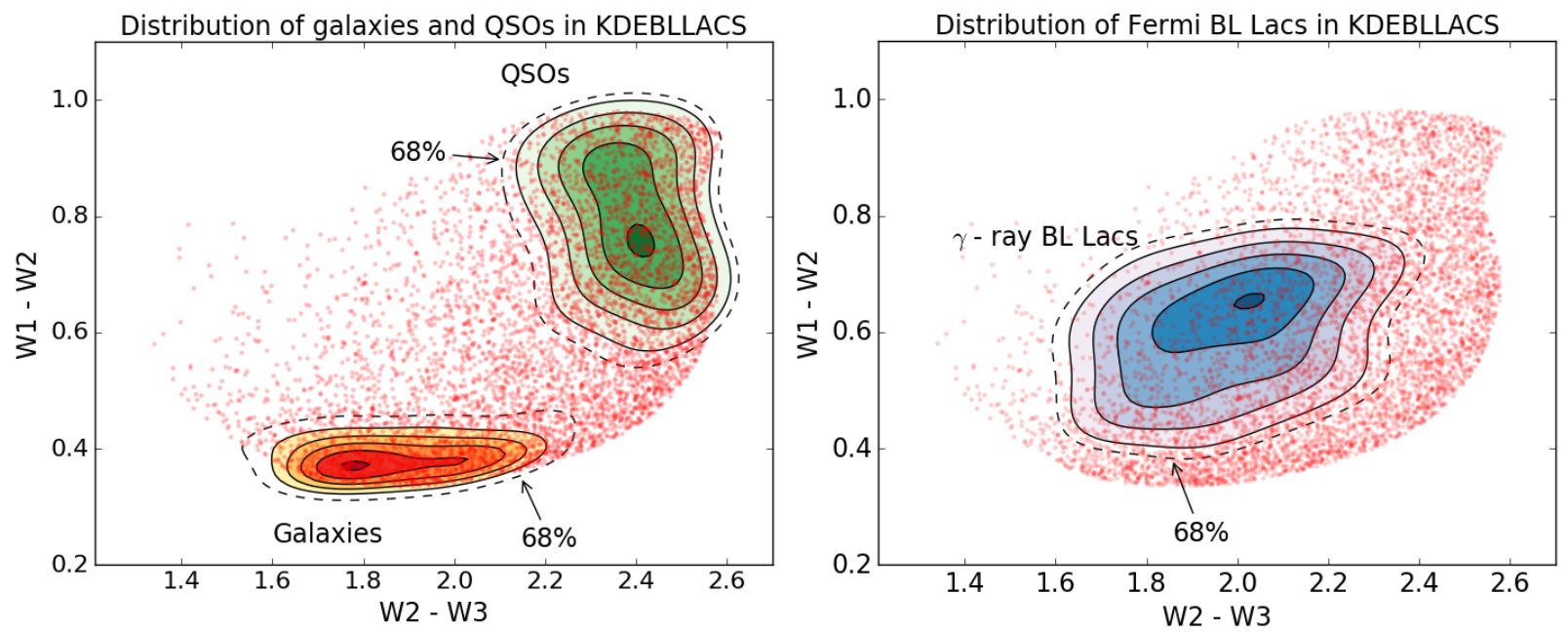

Figure 6.7: Contamination of KDEBLLACS. Left: The main contaminants of KDEBLLACS are normal galaxies and QSOs, both located in the edges of the sample. Right: PDF of the $\gamma$-ray BZBs available in 4FGL obtained with a KDE. We can see that the majority of BZBs are indeed located outside of the two contaminated zones. The solid lines represent the KDE isodensity contours, while the dashed lines represents the $68 \%$ isodensity contour.

\subsubsection{Selection effects}

Since $\sim 52 \%$ of WIBRaLS2 and $\sim 75 \%$ of KDEBLLACS sources in the footprint of SDSS have no available spectrum, we investigated possible selection effects, starting by comparing the typical optical colors of sources in WIBRaLS2 and KDEBLLACS with the colors of sources spectroscopically observed by SDSS, split by their SDSS spectroscopic classification. The lines in Figure 6.8 show the $90 \%$ and $50 \%$ isodensity contours for 10000 spectroscopically classified Galaxies (red), QSOs (green) and Stars (black), randomly selected from SDSS DR15. Overall, the candidates from WIBRaLS2 and KDEBLLACS catalog occupy very similar region of the $u-g$ vs $g-r$ optical diagram (Figure 6.8). Most of the WIBRaLS2 and KDEBLLACS candidates are located within the $90 \%$ isodensity contours defined by spectroscopic SDSS QSOs and Galaxies, which significantly overlap 
with the $90 \%$ contour for stars. In particular, in the case of WIBRaLS2 candidates (left panel), a large fraction (48\%) of candidates lie within the $50 \%$ contour of sources classified as QSOs, although other sources are scattered in the Galaxies and Stars-dominated areas. WIBRaLS2 candidates classified as WBZQ unsurpisingly peak in the area occupied by spectroscopically confirmed SDSS QSOs, while intermediate (WMIXED) and WBZB sources spread a much larger area consistent with both SDSS spectroscopic Stars and Galaxies. KDEBLLACS candidates are more evenly scattered through the isodensity contours for all three spectroscopic classes, indicating a likely larger contamination from the host galaxies. Similar behaviors are visible in the other SDSS color-color diagrams, not reported in Figure 6.8. In general, we can rule out the existence of significant selection effects due to the colors of the optical counterparts of our candidates.
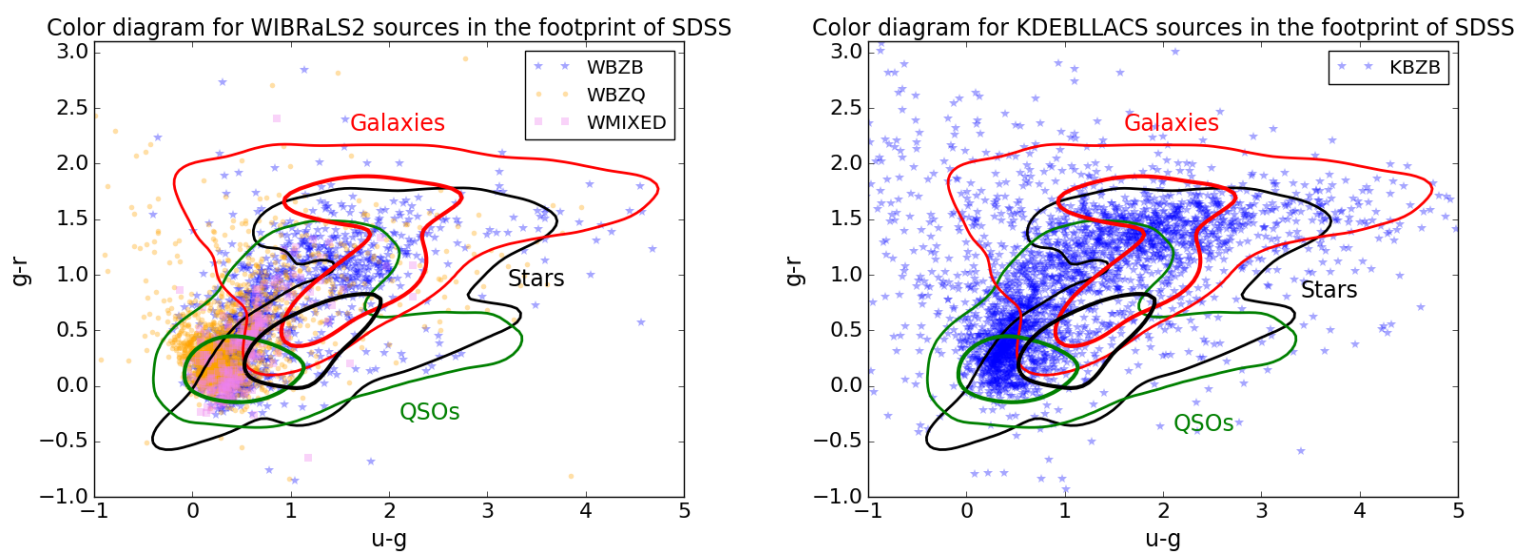

Figure 6.8: Optical color-color diagrams for WIBRaLS2 (left panel) and KDEBLLACS (right panel) sources in the footprint of SDSS. The total number of sources is 3407 for WIBRaLS2 and 2807 for KDEBLLACS, and are divided in WBZBs/KBZBs (blue stars), WBZQs (orange circles) and WMIXEDs (violet squares), accordingly to their classification in WIBRaLS2 and KDEBLLACS. The red, green and black thin and thick lines represent, respectively, the $90 \%$ and $50 \%$ isodensity contours based on the distribution of 10000 Galaxies, 10000 QSOs and 10000 Stars with available spectra in SDSS DR15.

Selection effects caused by the magnitude distribution of our candidates, however, are of major importance. As shown in Figure 6.9, a large majority of the candidates missing optical spectra, especially for KDEBLLACS catalog, are fainter than the candidates that have been followed-up spectroscopically. In particular, the reason why KDEBLLACS is spectroscopically less complete than WIBRaLS2 is because this catalog has in general fainter sources. This effect is expected as KDEBLLACS candidates appear to be intrinsically fainter than WIBRaLS2 sources in all three WISE bands (W1, W2 and W3) where they are detected, as shown in Figure 7 from D'Abrusco et al. (2019). The magnitude distributions of WIBRaLS2 candidates with and without SDSS spectroscopic counterparts (left panel 
in Figure 6.9), are very similar. This suggests that other selection effects, likely based on the colors, extension and morphology of the sources, increase the number of WIBRaLS2 sources lacking SDSS spectroscopic counterparts ${ }^{3}$.
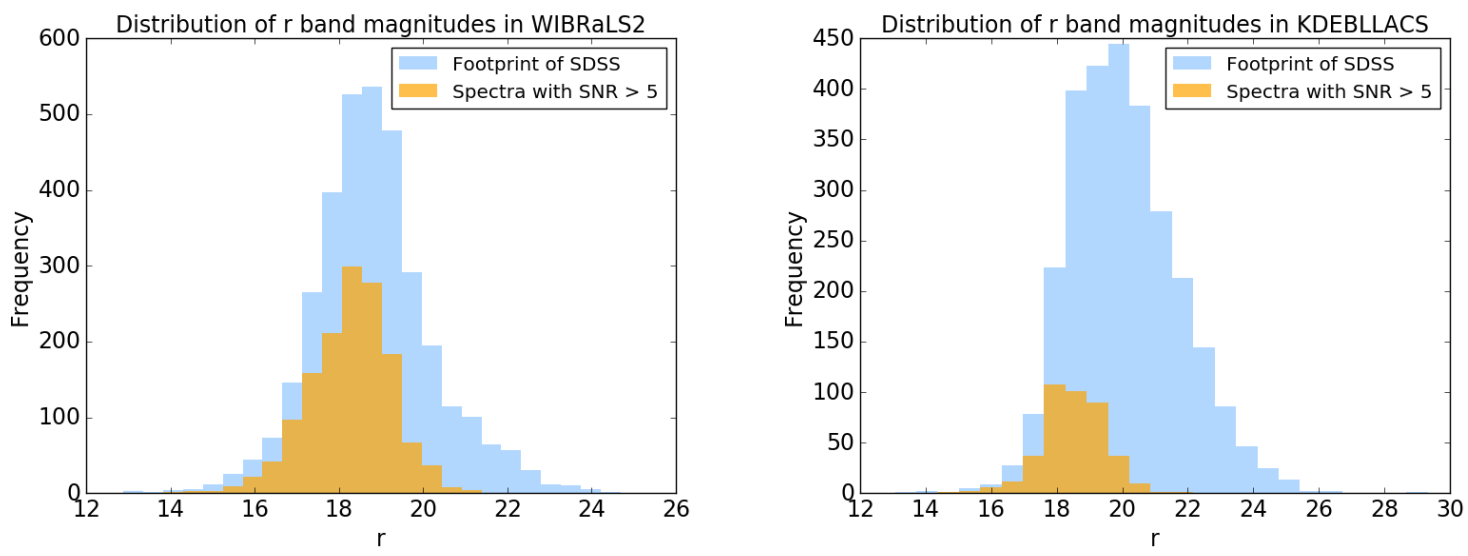

Figure 6.9: Distribution of $\mathrm{r}$ band magnitudes for WIBRaLS2 (left panel) and KDEBLLACS (right panel). The blue histograms stand for all WIBRaLS2 and KDEBLLACS sources in the footprint of SDSS (3407 and 2807 , respectively), while the orange histograms are only for sources with high quality $(\mathrm{S} / \mathrm{R} i \mathrm{5})$ spectra in SDSS DR15.

\subsubsection{Blazar candidates of uncertain type}

Some sources in the Fermi-LAT catalogs are considered blazar candidates of uncertain type (BCUs) because the adopted association methods select a counterpart that satisfies at least one of the following conditions (Ackermann et al., 2015; Abdollahi et al., 2020): i) An object classified as blazar of uncertain or transitional type in Roma-BZCAT. ii) A source with multiwavelength data indicating a typical two-humped blazar-like spectral energy distribution (SED) and/or a flat radio spectrum. BCUs are divided into three sub-types (Ackermann et al., 2015):

- BCU I: the counterpart has a published optical spectrum which is not sensitive enough for classifying it as BZQ or BZB.

- BCU II: there is no available optical spectrum but an evaluation of the SED synchrotron peak position is possible.

- BCU III: the counterpart shows typical blazar broadband emission and a flat radio

\footnotetext{
${ }^{3}$ For a general picture of the SDSS selection criteria for spectroscopic observations, see https: //www . sds. org/dr15/algorithms/legacy_target_selection/
} 
spectrum, but lacks a optical spectrum and reliable measurement of the synchrotron peak position.

In 4FGL, 1155 sources are considered as BCUs. Our analysis based on the optical spectra available in SDSS DR15 (Section 6.3) allowed us to give a conclusive classification for 11 of them, as shown in Table 6.1.

The 4FGL catalog associated the source 4FGL J0038.7-0204 with the broad line radio galaxy (RDG) 3C 17 at redshift $\sim 0.22$ as previously determined by Schmidt (1965) in agreement with the SDSS spectrum we analyzed. The kiloparsec-scale radio morphology of 3C 17 is dominated by a single-sided, curved jet (Morganti et al., 1993) also shining in the X-rays (Massaro et al., 2009, 2010). Recent optical spectroscopic observations also revealed that $3 \mathrm{C} 17$ is the brightest cluster galaxy in its large scale environment (Madrid et al., 2018).

\begin{tabular}{c|c|c|c} 
WISE name & 4FGL name & Class & $\mathrm{z}$ \\
\hline J003719.15+261312.6 & J0037.9+2612 & BZG & 0.1477 \\
J003820.53-020740.5 & J0038.7-0204 & RDG & 0.2204 \\
J013859.14+260015.7 & J0139.0+2601 & BZB & - \\
J020239.94-030207.9 & J0202.6-0258 & BZQ & 1.3444 \\
J084734.29+460928.0 & J0846.9+4608 & BZQ & 1.2165 \\
J094452.09+520233.4 & J0945.2+5200 & BZQ & 0.5630 \\
J095608.57+393515.8 & J0956.0+3936 & BZQ & 1.1730 \\
J130407.31+370908.1 & J1304.0+3704 & BZB & - \\
J134243.61+050432.1 & J1342.7+0505 & BZG & 0.1365 \\
\hline J123124.08+371102.2 & J1230.9+3711 & BZG & 0.2180 \\
J154150.09+141437.6 & J1541.7+1413 & BZG & 0.2230
\end{tabular}

Table 6.1 - Spectral classification for 11 sources classified as BCUs in 4FGL. This table is segmented in WIBRaLS2 (upper part) and KDEBLLACS (bottom). The columns give the name of the sources as in WISE and 4FGL, our optical classification and the redshifts as in SDSS DR15.

\subsubsection{New BZBS}

During the spectroscopic classification (Section 6.3), we found a total of 25 (10 in WIBRaLS2 and 15 in KDEBLLACS) sources with featureless optical spectra and a relatively strong blue continuum - typical characteristics of BZB non-thermal emission - which are not available in Roma-BZCAT. Their smoothed spectra, names/positions and $r$ band magnitudes are shown in Figure 6.10 

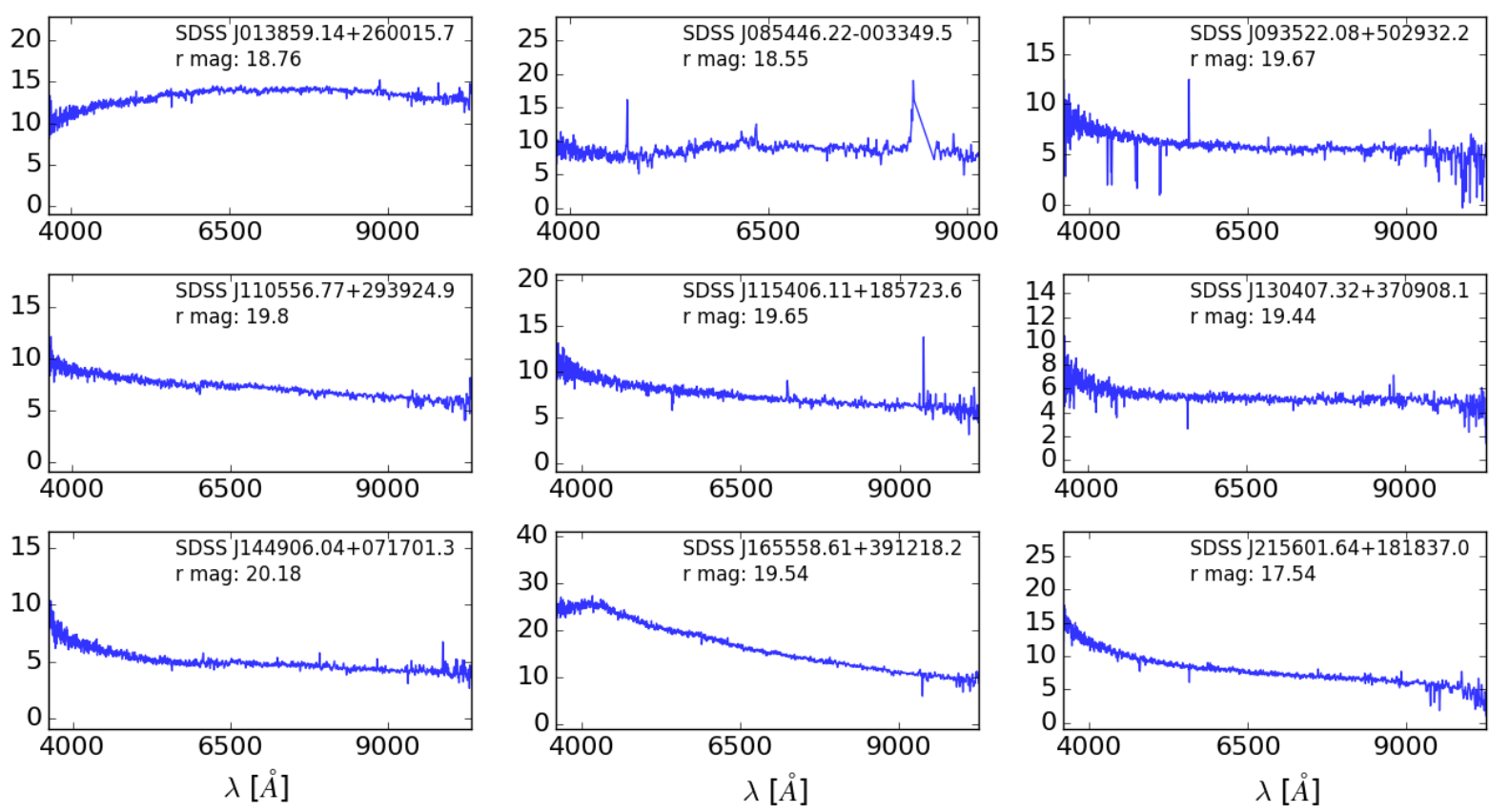

Figure 6.10: SDSS smoothed optical spectra of the new BZBs in WIBRaLS2 and KDEBLLACS. All sources present featureless spectra with color index $u-r<1.4$. We also show their SDSS name and $\mathrm{r}$ magnitude. The full version of this Figure can be found in de Menezes et al. (2019).

To check these new BZBs, we considered the $u-r$ color index of the SDSS photometric system (AB magnitudes), which is an efficient discriminator between BZBs and nuclei with weak or even absent activity (Massaro et al., 2012). When computing these indexes, we considered the extinction-corrected SDSS model magnitudes accordingly to the following formula:

$$
(u-r)=(u-r)_{o b s}-0.81 A_{r}
$$

where $A_{r}$ is the extinction in the $\mathrm{r}$ band, given in the SDSS database. Typically, BZBs present a color index $u-r \leq 1.4$ (Massaro et al., 2012), however, this criterium is not suited for sources at high redshifts $(\mathrm{z}>0.5)$. Table 6.2 summarizes our findings.

\subsection{Summary and conclusions}

Here we characterized the two newly released catalogs of blazar candidates WIBRaLS2 and KDEBLLACS based on 1830 optical spectra - 1798 spectra with $S / N>5$ and 32 with $S / N<5$ but with a counterpart in Roma-BZCAT - available in SDSS DR15 data. Both catalogs indeed presented a high number of spectroscopic confirmed blazars, although the contamination level of WIBRaLS2 was 60\%, mainly due to QSOs, and the contamination 


\begin{tabular}{c|c|c} 
WISE name & SDSS name & $u-r$ \\
\hline J013859.14+260015.7 & J013859.14+260015.7 & 1.09 \\
J085446.24-003348.1 & J085446.22-003349.5 & 0.82 \\
J093522.08+502932.2 & J093522.08+502932.2 & 0.88 \\
J115406.13+185723.6 & J115406.11+185723.6 & 1.16 \\
J130407.31+370908.1 & J130407.32+370908.1 & 1.11 \\
J144906.05+071701.2 & J144906.04+071701.3 & 0.86 \\
J165558.59+391218.1 & J165558.61+391218.2 & 0.91 \\
J215601.64+181837.1 & J215601.64+181837.0 & 0.45 \\
J220812.70+035304.5 & J220812.70+035304.6 & 0.85 \\
J235915.62+221450.0 & J235915.62+221450.1 & 1.38 \\
\hline J000710.65-032029.4 & J000710.65-032029.4 & 0.74 \\
J020303.61-024547.3 & J020303.62-024546.9 & 0.82 \\
J024024.36-025334.3 & J024024.37-025334.3 & 0.70 \\
J083706.00+583152.9 & J083706.01+583152.9 & 0.60 \\
J083955.10+121702.9 & J083955.09+121703.0 & 0.71 \\
J091804.13+071653.6 & J091804.13+071653.6 & 1.22 \\
J101147.54+360018.7 & J101147.50+360018.8 & 0.68 \\
J103852.20+325651.7 & J103852.17+325651.6 & 1.22 \\
J111356.24+552255.3 & J111356.30+552255.6 & 0.60 \\
J114221.77+334201.8 & J114221.76+334201.8 & 0.85 \\
J114352.67+155821.9 & J114352.66+155822.0 & 0.51 \\
J135154.45+285008.0 & J135154.45+285007.9 & 1.26 \\
J154714.91+265442.2 & J154714.91+265442.4 & 0.77 \\
J171747.84+392607.3 & J171747.85+392607.3 & 0.99 \\
J171841.42+360522.6 & J171841.44+360522.2 & 1.32
\end{tabular}

Table 6.2 - New BZBs found during the spectroscopic classification described in Section 6.3. The first and second columns present the name of the objects in WISE and SDSS. The last column shows the color index $u-r$ based on SDSS AB magnitudes. The upper section of the table shows the BZBs found in WIBRaLS2 while the bottom section shows the ones found in KDEBLLACS.

of KDEBLLACS was $\sim 70 \%$, mainly due to QSOs and normal galaxies. We stress that these contamination estimates represent upper limits due to the selection effects impacting the SDSS spectroscopic sample used in this paper, as described in Section 6.4.3. Our main results can be summarized as follows:

- Including the objects in Roma-BZCAT, $34.6 \%, 27.7 \%$ and $42.5 \%$ of the sources respectively in WBZB, WBZQ and WMIXED are blazars, which gives an overall weighted lower limit to the efficiency of WIBRaLS2 blazar selection of $\approx 31 \%$, with the major contaminants being, as expected, QSOs.

- The lower limits to the efficiency of KDEBLLACS in identifying blazars is $\sim 30 \%$, and its contaminants - mainly galaxies and QSOs - are concentrated on the edges 
of the mid-IR color-color diagram (Figure 6.7).

- The spectral analysis carried on in Section 6.3 led us to the discovery of 25 new BZBs not available anywhere else in literature and to the classification of 11 BCUs listed in 4 FGL.

This work contributes to a better understanding of the $\gamma$-ray sky in the Fermi-LAT era. In particular, the community will benefit from the characterization of WIBRaLS2 and KDEBLLACS in population studies of blazars and in subsequent programs of spectroscopic follow-up needed to confirm the nature of the UGSs.

\section{Acknowledgements}

We thank the anonymous referee for constructive comments which helped to improve the manuscript. The accomplishment of this project was only possible due to the ongoing support from the São Paulo Research Foundation (FAPESP), grants 2016/25484-9, 2018/24801-6 and 2017/01461-2. PH acknowledges support from the CONACyT program for Ph.D. studies. FR acknowledges support from FONDECYT Postdoctorado 3180506 and CONICYT Chile grant Basal-CATA PFB-06/2007. R.D'A. is supported by NASA contract NAS8-03060 (Chandra X-ray Center). The full acknowledgments can be found in the original paper by de Menezes et al. (2019). 
Chapter 7

\title{
On the association of gamma-ray sources
}

\author{
This thesis chapter is a shortened version of the paper "On the phy- \\ sical association of Fermi-LAT blazars with their low-energy counterparts" by \\ de Menezes et al. (2020), published in the Astrophysical Journal Supplement \\ Series, volume 248, number 2. The author was responsible for writing the paper \\ and developing both algorithms for counterpart association. The original work \\ can be found online here.
}

\section{Abstract}

Associating $\gamma$-ray sources to their low-energy counterparts is one of the major challenges of modern $\gamma$-ray astronomy. In the context of the Fourth Fermi Large Area Telescope Source Catalog (4FGL), the associations rely mainly on non-physical parameters, as apparent magnitude, integrated flux, and angular separation between the $\gamma$-ray source and its low-energy candidate counterpart. In this work we propose a new use of likelihood ratio and a complementary supervised learning technique to associate $\gamma$-ray blazars in 4FGL, based only on spectral parameters as $\gamma$-ray photon index, mid-infrared colors and radio-loudness. In the likelihood ratio approach, we crossmatch the WISE Blazar-Like Radio-Loud Sources catalog with 4FGL and compare the resulting candidate counterparts with the sources listed in the $\gamma$-ray blazar locus to compute an association probability for 1138 counterparts. In the supervised learning approach, we train a random forest algorithm with 869 high confidence blazar associations and 711 fake associations, and then compute an association probability for 1311 candidate counterparts. A list with all 4FGL blazar candidates of uncertain type associated by our method is provided to guide future 
optical spectroscopic follow up observations.

\subsection{Introduction}

The association of $\gamma$-ray sources with their low-energy counterparts is a long-standing challenge since the beginning of modern $\gamma$-ray astronomy (Fichtel et al., 1994). Its major underlying difficulty is related to the large positional uncertainty of $\gamma$-ray observations. Even in the era of the Fermi Large Area Telescope (LAT; Atwood et al., 2009), the positional uncertainty of $\gamma$-ray sources range from a couple of arcminutes up to $\sim 1^{\circ}$ (Abdollahi et al., 2020). Then, according to the recent release of the Fermi-LAT Fourth Source Catalog (4FGL; Abdollahi et al., 2020), 25\% of its sources lack an assigned low energy counterpart and thus have uncertain nature. Although the fraction of unassociated $\gamma$-ray sources (UGSs) is still large, 4FGL presents a modest improvement in comparison with its previous releases, which had $\sim 30 \%$ of unassociated sources (Abdo et al., 2010; Nolan et al., 2012; Acero et al., 2015). The largest population of associated $\gamma$-ray sources is dominated by pulsars, pulsar wind nebulae and supernova remnant in the Galactic plane, and by blazars in the extragalactic sky (Abdollahi et al., 2020)

Several studies searching for the counterparts of $\gamma$-ray sources have been performed in the past decade (Abdo et al., 2010; Nolan et al., 2012; Acero et al., 2015). Dedicated follow up observations at radio, infrared and X-rays allowed for the identification of potential counterparts for several Fermi-LAT sources, for which optical spectra were then collected to establish their nature (Massaro et al., 2015, 2016a; Peña-Herazo et al., 2017; Marchesini et al., 2019; Peña-Herazo et al., 2019). Once a candidate counterpart is found, it can be considered in the association methods of the Fermi-LAT catalogs. All association methods used by the Fermi-LAT Collaboration (Abdollahi et al., 2020) consist in computing the association probability (AP) based mainly on the angular separation between the center of the $\gamma$-ray source and the position of its candidate counterpart, thus being mostly a geometrical approach and neglecting physical properties of candidate counterparts, such as colors, spectral shape and radio-loudness, to name a few. When a candidate counterpart has AP $>80 \%$ in 4FGL, the source is considered associated.

In this work we propose two different association procedures, both independent of angular separation and relying mainly on the $\gamma$-ray-mid-infrared connection of candidate 
blazar counterparts selected from the WISE Blazar-Like Radio-Loud Sources catalog (WIBRaLS; D'Abrusco et al., 2019). WIBRaLS is a catalog of radio-loud candidate blazars whose WISE mid-infrared (MIR) colors are selected to be consistent with MIR colors of confirmed $\gamma$-ray emitting blazars (Massaro et al., 2011b; D'Abrusco et al., 2012). We compute the APs with two different methods: i) the likelihood ratio (LR; Sutherland and Saunders, 1992), already adopted by the Fermi-LAT Collaboration, but with a different setup; and ii) a random forest algorithm (RF; Breiman, 2001). By assigning an AP to each candidate counterpart, we can schedule/program future optical spectroscopic follow-ups on these results, prioritizing those targets with higher AP.

Computing an AP for each counterpart candidate is crucial because all methods used to select candidate blazars are statistical in nature and do not take into account the specific $\gamma$-ray properties of each Fermi source to be associated but only collective features of a population of sources (in our case, the $\gamma$-ray emitting confirmed blazars in the blazar locus. See $\$ 7.2)$. Thus, association methods bridge the gap between collective behavior and each specific case, giving an intra-source prioritization - should there be multiple candidates for the same $\gamma$-ray source - and an inter-source prioritization to maximize the effectiveness of spectroscopic follow-ups.

Machine learning techniques have been used before in the context of Fermi-LAT catalogs to i) predict the spectral class of UGSs (Doert and Errando, 2014; Parkinson et al., 2016; Lefaucheur and Pita, 2017; Salvetti et al., 2017) based only on the $\gamma$-ray properties available in the Second and Third Fermi Source Catalogs (2FGL and 3FGL, respectively; Nolan et al., 2012; Acero et al., 2015); ii) predict the nature of blazar candidates of uncertain type (BCUs; Hassan et al., 2012; Chiaro et al., 2016; Kovačević et al., 2019); iii) and even for spotting candidates of dark matter Galactic subhalos (Mirabal et al., 2012). This is the first time, however, that machine learning is used to associate Fermi-LAT sources with their low-energy counterparts.

The idea underlying this work is that $\gamma$-ray blazars have some specific radio-MIR characteristics which can tell them between, e.g., two counterpart candidates lying in the same elliptical uncertainty region of a $\gamma$-ray source. Indeed, some sources associated in 4FGL have a very high AP with counterparts that are not the closest ones if compared with sources listed in the latest version of WIBRaLS (D'Abrusco et al., 2019). This can happen simple because the latest version of WIBRaLS was not taken into account when 
associating 4FGL sources, but highlights a possible bias in a method that associate sources based mainly on angular separation: if the real $\gamma$-ray source counterpart is not listed in one of the catalogs used by the association algorithms, then the method will simply choose the closest candidate.

The paper is organized as follows. In $\S 7.2$ and $\S 7.3$ we describe the samples used to carry out our analysis, providing basic details on both WIBRaLS and 4FGL. In $\S 7.4$ and $\S 7.5$ we describe the adopted methods followed by the achieved results in $\S 7.6$ and $\S 7.7$. We conclude and summarize our work in $\S 7.8$. The WISE magnitudes are in the Vega system and are not corrected for the Galactic extinction, since, as shown in D'Abrusco et al. (2014), such correction only affects the magnitude at $3.4 \mu \mathrm{m}$ for sources lying close to the Galactic plane and it ranges between $2 \%$ and $5 \%$ of the magnitude, thus not affecting significantly the results. WISE bands are indicated as W1, W1, W3 and W4, and correspond respectively to the nominal wavelengths at $3.4,4.6,12$, and $22 \mu \mathrm{m}$, while the colors are defined as $c_{12}=W 1-W 2, c_{23}=W 2-W 3$ and $c_{34}=W 3-W 4$. The adopted MIR radio-loudness parameter is defined as $q_{22}=\log \left(S_{22 \mu m} / S_{\text {radio }}\right)$, where $S_{22 \mu m}$ is the flux density in the WISE W4 band, and $S_{\text {radio }}$ is the radio flux density at $1.4 \mathrm{GHz}$ or at $843 \mathrm{MHz}$, depending on the radio survey in which the WIBRaLS radio counterpart is identified (see D'Abrusco et al., 2019, for more details).

\subsection{Samples used for the likelihood ratio method}

We apply the LR method to all 1311 sources listed in 4FGL with at least one counterpart in WIBRaLS, comparing their MIR colors and radio-loudness with those of the $\gamma$-ray blazars lying within the blazar locus (D'Abrusco et al., 2013).

The blazar locus is defined by a sample of confirmed Fermi-LAT blazars listed in RomaBZCAT (Massaro et al., 2015) and associated with WISE counterparts detected in all four filters W1, W2, W3 and W4. The locus is modeled in a three-dimensional space generated by the principal components of the MIR color-color-color distribution and describes the typical MIR colors of $\gamma$-ray blazars, thus being ideal for our purposes (see, e.g., D'Abrusco et al., 2013, 2019). 

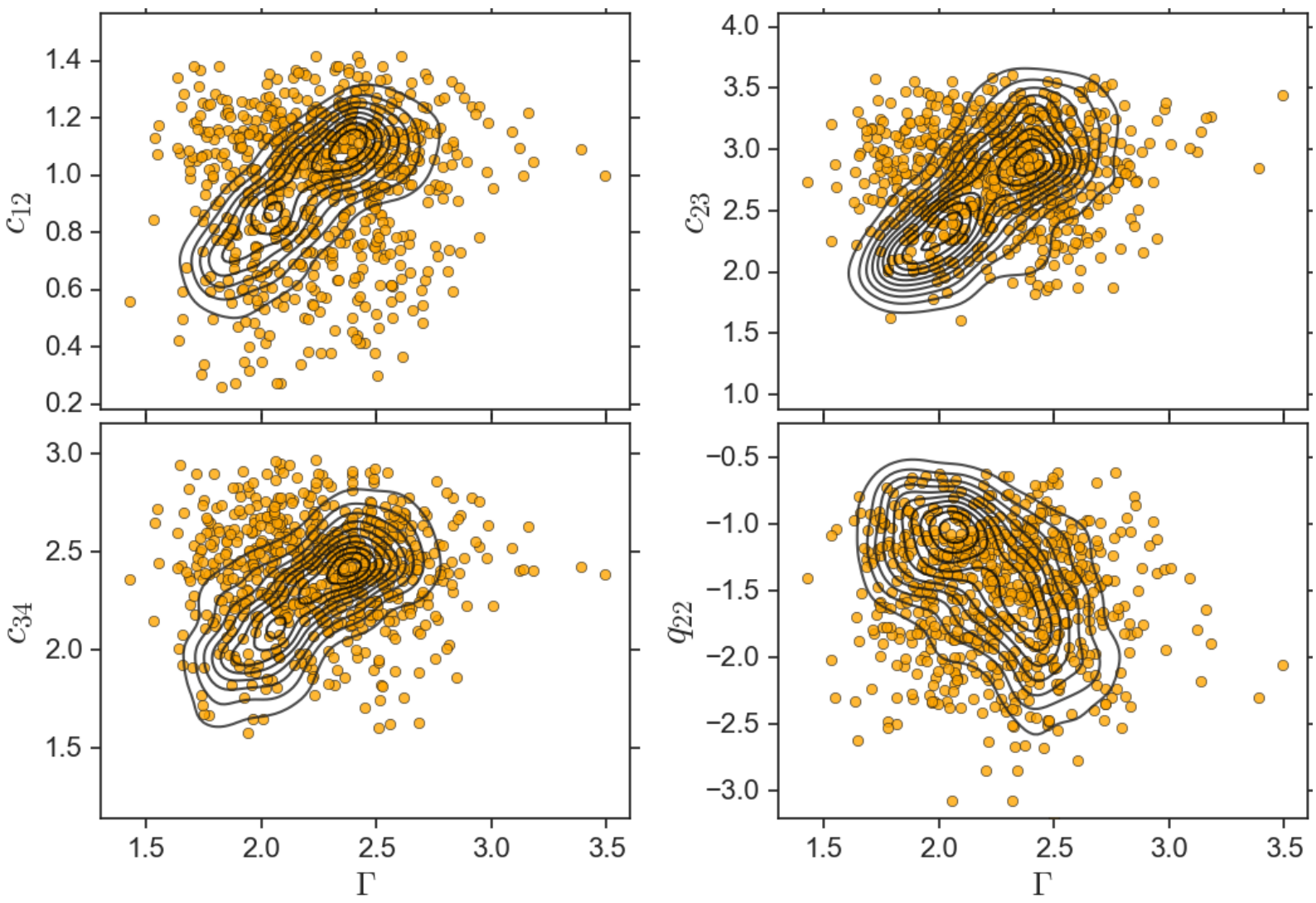

Figure 7.1: Training sample for the RF algorithm. The contours represent the distribution of the 869 high confidence associations, while the orange dots represent the fake associations. The spectral parameters for the training sample are shown in terms of the $\gamma$-ray photon index, $\Gamma$, the three MIR colors $c_{12}, c_{23}$ and $c_{34}$ (as defined in the last paragraph of $\$ 7.1$ ) and the MIR radio-loudness $q_{22}$.

\subsection{Samples used for the supervised learning method}

The training set of supervised learning algorithms needs to be representative of the expected distribution of outcomes in the test set, or the sample on which the trained algorithm will be applied. In this case, we train a RF algorithm by gathering high-confidence associations and not-associated (fake) counterparts. We do it by selecting all associated sources in 4FGL having a counterpart in WIBRaLS and with $\gamma$-ray statistical significance above $10 \sigma$. Such high confidence $\gamma$-ray detections tend to have smaller error ellipses, and for this reason they are easier to correctly associated with low energy counterparts. The final number of high confidence associations in the training set was 869. The fake associations were selected as the WIBRaLS sources not associated in 4FGL but lying within $0.5^{\circ}$ from 4FGL sources that are already associated with a low-energy counterpart, resulting in a total of 711 fake counterparts for the training sample. It is possible that a few of these fake associations are the real counterparts of the $\gamma$-ray sources. 
The panels in Figure 7.1 show the differences between high confidence associations (contourns) and fake associations (orange dots) in the training sample. Based on these $\gamma$-ray-MIR characteristics, supervised learning algorithms can be trained to tell how likely a WIBRaLS source is associated as the counterpart of a $\gamma$-ray source. Once trained, we applied the RF method to a test sample made of the 1311 4FGL-WIBRaLS crossmatches, the same sample used in the LR method.

\subsection{The likelihood ratio method}

The LR technique was first used in the context of source association by Richter (1975) and has been introduced and developed to look for possible counterparts among faint radio, infrared and X-ray sources (Wolstencroft et al., 1986; Sutherland and Saunders, 1992; Masci et al., 2001). In the context of Fermi-LAT, the LR between a candidate counterpart $i$ within the error ellipse of a 4FGL source $j$ is computed as (Ackermann et al., 2011; Ajello et al., 2020):

$$
L R_{i j}=\frac{e^{-r_{i j}^{2} / 2}}{N A},
$$

where $r_{i j}=\theta /\left(\sigma_{i}^{2}+\sigma_{j}^{2}\right)^{1 / 2}$ is the normalized angular separation between the $\gamma$-ray source $j$ and candidate counterpart $i$, with $\theta$ being the angular separation between the counterpart and the center of the $\gamma$-ray source, $\sigma_{i}$ being the low-energy positional uncertainty and $\sigma_{j} \equiv \sigma_{95 \%} / 2.4477$ being the normalized geometric mean of the semi-major and semi-minor axes of the $95 \%$ FGL confidence error ellipse. $N$ is the surface density of objects brighter than the candidate $i$ and $A$ is the solid angle encompassed by the $95 \%$ confidence LAT error ellipse. Below we modify this method by taking into account only spectral properties of the candidate counterparts, as MIR colors and radio-loudness, and comparing these properties with what is expected for $\gamma$-ray blazars lying in the blazar locus (D'Abrusco et al., 2013). In $\S 7.6 .1$ we add a dependence on the angular separation to this method to measure the impact of angular separation in our results.

The LR method we adopt to estimate the AP is a modification of the LR method described in Sutherland and Saunders (1992) and Ackermann et al. (2011). The adopted steps are as follows.

1. We crossmatch WIBRaLS with 4FGL to create the list of all $\gamma$-ray blazar candidates 
that lie within the positional uncertainty region at $95 \%$ level of confidence of each Fermi-LAT object. With a total of 1311 potential counterparts, the result of this crossmatch correspond to all of the 4FGL candidate counterparts listed in WIBRaLS.

2. For each 4FGL source, we consider the $\gamma$-ray photon index interval $\Delta \Gamma=[\Gamma-$ $\left.\sigma_{\Gamma}, \Gamma+\sigma_{\Gamma}\right]$, where $\sigma_{\Gamma}$ is the uncertainty on the photon index as listed in $4 \mathrm{FGL}$. Then, given the correlations between $\Gamma$, the MIR colors $c_{12}$ and $c_{34}$, and the MIR radioloudness $q_{22}$ for the blazars in the $\gamma$-ray blazar locus (D'Abrusco et al., 2012), we select from WIBRaLS only those sources with parameters within the blazar locus ranges $\Delta c_{12}=\left[c_{12}^{\min }, c_{12}^{\max }\right], \Delta c_{34}=\left[c_{34}^{\min }, c_{34}^{\max }\right]$ and $\Delta q_{22}=\left[q_{22}^{\min }, q_{22}^{\max }\right]$ corresponding to the $\Delta \Gamma$ interval, as shown in Figure 7.2. This step reduces the total number of candidate counterparts from 1311 to 1138.

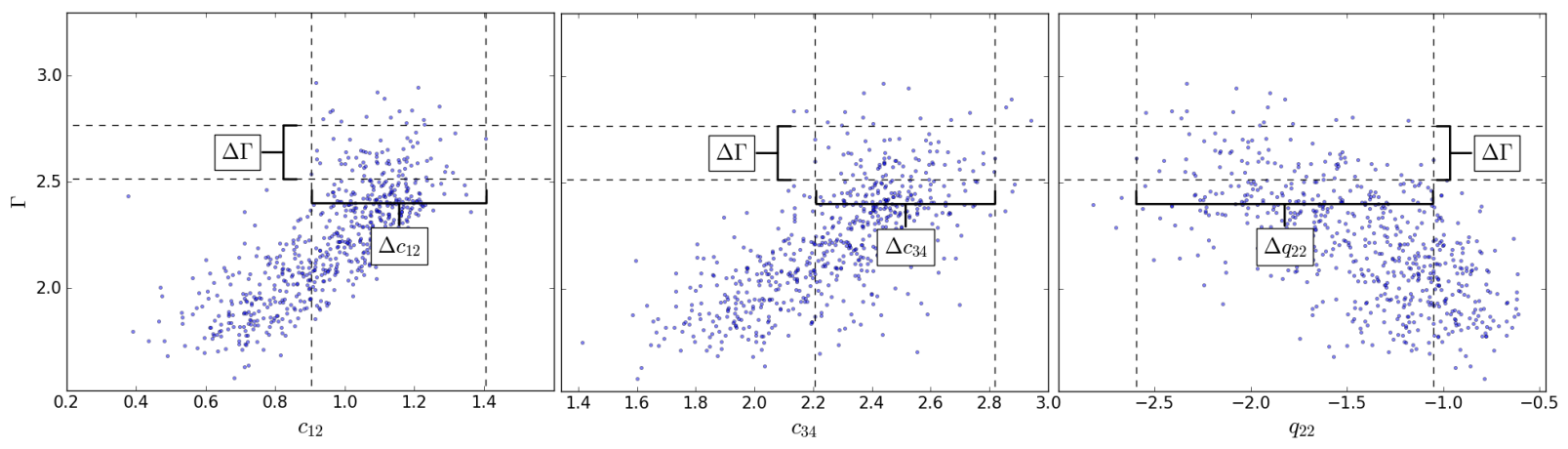

Figure 7.2: Region selected in the blazar locus for a source with $\gamma$-ray photon index $\Gamma=2.65 \pm 0.10$. Given an interval $\Delta \Gamma$, only the sources within the ranges $\Delta c_{12}, \Delta c_{34}$ and $\Delta q_{22}$ can be selected as possible counterparts of $\gamma$-ray sources.

3. For each MIR counterpart $i$ of a 4FGL source $j$ we compute the LR using the following equation:

$$
\log L R_{i, j}=\log \left[\frac{1}{2 \pi \sigma_{1} \sigma_{2}} \frac{Q\left(\Delta c_{12}, \Delta c_{34}, \Delta q_{22}\right)}{N\left(\Delta c_{12}, \Delta c_{34}, \Delta q_{22}\right)}\right]
$$

where $\mathrm{Q}\left(\Delta c_{12}, \Delta c_{34}, \Delta q_{22}\right)$ is the probability to find a WIBRaLS source with MIR colors and radio-loudness within the ranges $\Delta c_{12}, \Delta c_{34}$ and $\Delta q_{22}$ over the entire WIBRaLS catalog (i.e., it is the ratio between the total number of sources lying within the specified intervals and the total number of WIBRaLS sources), and $\mathrm{N}\left(\Delta c_{12}, \Delta c_{34}, \Delta q_{22}\right)$ is the local surface density of WIBRaLS sources within a circle of $8^{\circ}$ radius centered in the $4 \mathrm{FGL}$ source and having MIR colors and radio-loudness within the specified ranges (i.e., it is the surface density of the background objects at the appropriate Galactic latitude). The positional uncertainty errors of the $95 \%$ con- 
fidence regions of $4 \mathrm{FGL}$ sources are given by $\sigma_{1}$ and $\sigma_{2}$. Angular separation between the $\gamma$-ray source and its counterpart is not taken into account.

4. To compute the AP based on the LR calculated above, we first generate 500 fake $\gamma$-ray catalogs by shifting the sky positions of each 4FGL source by a random value between $0.3^{\circ}$ and $3^{\circ}$ in a random direction of the sky. These small shifts in R.A. and Dec. guarantee that we preserve the inhomogeneity of the $\gamma$-ray sky, which has more sources concentrated towards the Galactic plane. We then repeat steps 2 and 3 above for all matches between the fake sources (belonging to the 500 generated catalogs) and WIBRaLS sources to compute the average distribution function $(<$ $G_{\text {fake }}\left(L R_{i, j}\right)>$ ) of fake $\log L R_{i, j}$. The $\log L R_{i, j}$ distributions for the real and fake matches are shown in blue and green, respectively, in Figure 7.3. As a matter of comparison, we overplot the $\log L R_{i, j}$ distributions when adding the exponential dependence of angular separation, $r$, shown in Equation 7.1, to our method (hatched distributions).

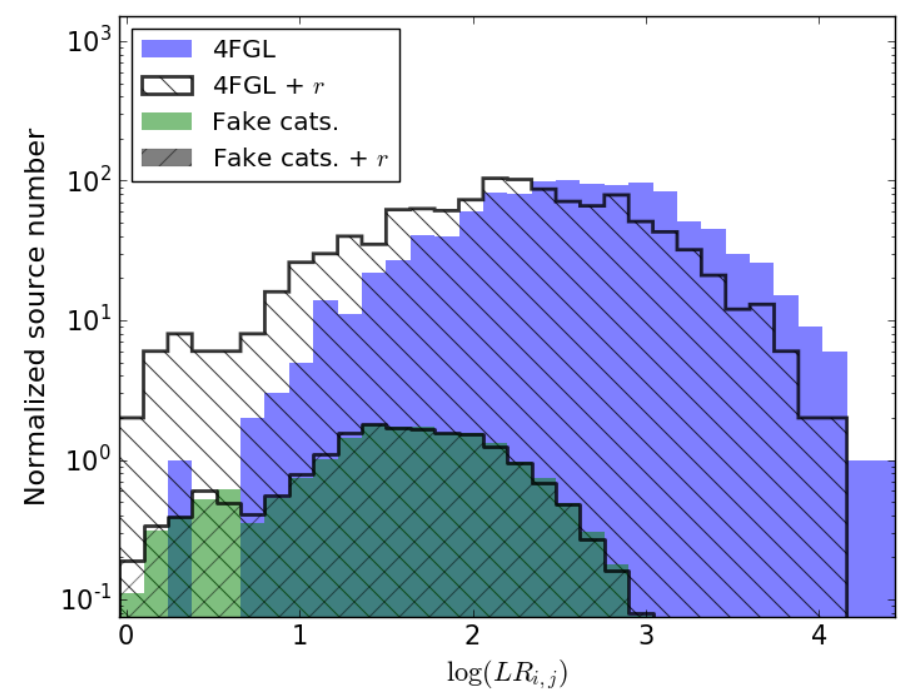

Figure 7.3: Distribution of the $\log \left(L R_{i, j}\right)$ for the crossmatches between WIBRaLS and 4FGL $\left(G_{\text {real }}\right)$, in blue; and average distribution for the crosmatches between WIBRaLS and the fake $\gamma$-ray catalogs $\left(<G_{\text {fake }}>\right.$ ), in green. The hatched histograms represent the same distributions when taking angular separation, $r$, into account with the exponential behaviour shown in Equation 7.1.

5. We then compare the real and the fake distributions of $\log L R_{i, j}$ to determine the reliability for the real associations by computing:

$$
\rho\left(L R_{i, j}\right)=1-\frac{<G_{\text {fake }}\left(L R_{i, j}\right)>}{G_{\text {real }}\left(L R_{i, j}\right)}
$$


where $\left\langle G_{\text {fake }}\left(L R_{i, j}\right)>\right.$ is the average $\log L R_{i, j}$ distribution for fake catalogs and $G_{\text {real }}\left(L R_{i, j}\right)$ is the $\log L R_{i, j}$ distribution for the real $\gamma$-ray source catalog, as shown in Figure 7.3. The reliability, $\rho$, computed according to this equation represents an approximate measurement of the AP for a potential counterpart having a given $\log L R_{i, j}$. In Figure 7.4 we show the dependence of $\rho$ on $\log \left(L R_{i, j}\right)$. Some fluctuations are observed for $\log \left(L R_{i, j}\right)<1.5$, but the overall behaviour of the curve is clear: for $\log \left(L R_{i, j}\right)>1.5$, basically all counterpart candidates have very high $(>95 \%)$ APs. The orange shadow in Figure 7.4 represent the uncertainty region caused by choosing different radii when computing the local surface density (see step 3 above). This region was generated by ranging the radii from $3.5^{\circ}$ up to $15^{\circ}$ in 200 linearly spaced intervals. We observe that, in the region defined by $\log \left(L R_{i, j}\right)>1.5$, the APs do not really depend on how we choose the background region radius.

The APs obtained with this method range from $60 \%$ to $100 \%$. Among the sources listed as identified in 4FGL-those for which the association to the low-energy counterpart is guaranteed-the APs are always above $99 \%$.

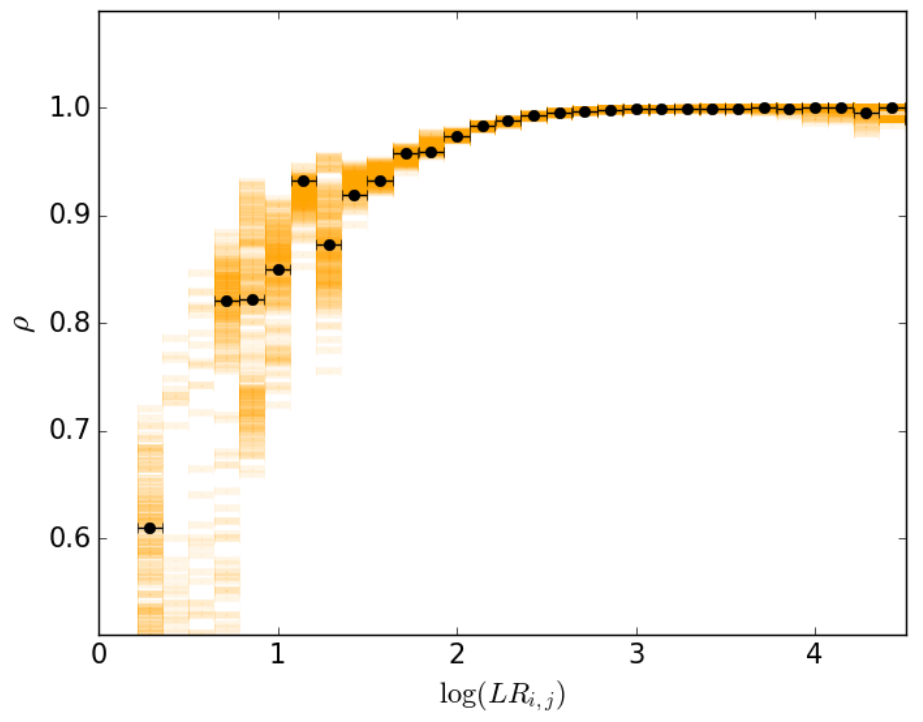

Figure 7.4: The reliability $\rho$ derived from the LR method as function of $\log \left(L R_{i, j}\right)$. The black dots are the points adopted in this work, while the orange shadow represents the uncertainty region due to different choices of radii when computing the local surface density $\mathrm{N}\left(\Delta c_{12}, \Delta c_{34}, \Delta q_{22}\right)$. In the region $\log \left(L R_{i, j}\right)>1.5$, the impact of choosing different radii is negligible. 


\subsection{The random forest method}

In the context of supervised learning algorithms, we use a RF (Breiman, 2001) to compute the AP of 4FGL counterparts. The RF method is an ensemble classifier that uses decision trees as building blocks for classification (James et al., 2013). For classifying a new object, each tree in the forest chooses one class and, by aggregating the predictions of all decision trees, the RF makes a final prediction based on the choice made by the majority of the trees, thus improving the predictive capability and reducing the tendency of standard decision trees to overfit the training sample.

We train and estimate the accuracy of the RF method with the sample described in $\S 7.3$ using cross-validation: the RF algorithm has been trained with 10 subsets, each containing $90 \%$ of the training sample (where the sources are randomly chosen), and testing it on the complementary 10 subsets containing $10 \%$ of the training sample, in a way that the final accuracy of $78.2 \% \pm 3.3 \%$ is simply the average ratio of correctly predicted observations to the total observations. The following attributes listed in 4FGL and WIBRaLS were used during the learning process: $\gamma$-ray power law photon index $\Gamma$, WISE colors $c_{12}, c_{23}$, and $c_{34}$, and radio-loudness $q_{22}$ computed with respect to the infrared flux in band W4. We tested several other parameters available in 4FGL and WIBRaLS, as $\gamma$-ray variability index and ratios between $\gamma$-ray flux densities (power law and logparabola spectral models) and radio flux density, but all of them presented a negligible impact on the results, with improvements in the algorithm accuracy of $\sim 3 \%$ in the best case (i.e., $\gamma$-ray variability index), and sometimes only adding noise. Among the used parameters, the one with largest impact in the accuracy when combined with $\Gamma$ is $c_{12}$, followed by $c_{34}, q_{22}$ and $c_{23}$, respectively. As discussed in $\S 7.1$, we are neglecting angular separation between the center of the $\gamma$-ray sources and the position of the MIR sources listed in WIBRaLS, as we are proposing an association method that relies only on spectral properties.

We use the RF classifier available in the Python library sklearn (Pedregosa et al., 2011). The code fits several decision trees on sub-samples of the dataset and average them to improve the predicted accuracy and prevent over-fitting. To guarantee the stability of our results, we use 5000 trees and let the nodes grow until all leaves contain less than the minimum number of samples required to split an internal node. The APs obtained with the RF algorithm range from $5 \%$ to $100 \%$ and the lowest AP obtained for blazars listed 
as identified in 4FGL (all of them included in the training sample) is $70 \%$.

\subsection{Results from the likelihood ratio approach}

The total number of associations with AP $>99 \%$ (i.e., as high as the AP obtained for the identified 4FGL sources. See $\S 7.4$ ) is 743 out of the 1138 original 4FGL-WIBRaLS crossmatches within the intervals $\Delta c_{12}, \Delta c_{34}$ and $\Delta q_{22}$. This number increases to 1051 when considering counterparts with AP $>95 \%$. Among all the 1138 sources for which we compute an AP, 283 are associated in 4FGL as BCUs, 473 as BL Lacs, 350 as flat spectrum radio quasars (FSRQs) and the rest of them are divided into a few non-blazar extragalactic and unknown objects. We found 5 UGSs with a counterpart in WIBRaLS and with APs ranging from $93 \%$ up to $99 \%$. The BCUs and UGSs associated here-specially those with APs as high as the identified 4FGL sources-are promising targets for future optical spectroscopic follow up missions. In fact, optical spectroscopic observations for some of them are available in de Menezes et al. (2020) and Peña-Herazo et al. (2020, in prep.), where all of the sources have blazar-like optical spectra. All BCUs and UGSs associated here are listed in Tables 7.2 and 7.3 in Appendix 7.9.

Among the $\gamma$-ray sources associated here (with any AP), 22 of them have different counterparts in 4FGL which are not listed in WIBRaLS (see Table 7.1). Probably due to the exponential dependence with angular separation (see Eq. 4.1) adopted in 4FGL, the 22 counterparts listed in 4FGL are generally closer to the center of the $\gamma$-ray sources than the WIBRaLS counterparts selected based on MIR colors. Furthermore, 165 of the 1138 WIBRaLS counterpart candidates are not associated by the LR method adopted in 4FGL. These sources were associated in 4FGL mainly via the Bayesian method (Abdollahi et al., 2020). Additionally, no correlation is observed between the APs computed here and the APs computed with the LR method adopted in 4FGL, indicating that the methods are completely independent.

\subsubsection{Considering angular separation}

When we consider angular separation (with an exponential dependence as shown in Equation 7.1) together with WISE MIR colors and $q_{22}$, the LR distributions look like the hatched histograms in Figure 7.3. As the distributions are very similar, the APs resulting 


\begin{tabular}{|l|l|l|r|r|}
\hline \multicolumn{1}{|c|}{ 4FGL name } & Association WIBRaLS & \multicolumn{1}{|c|}{ Association 4FGL } & AP LR WIB & AP LR 4FGL \\
\hline J0119.9+4053 & J011947.90+405418.4 & CRATES J012018+405314 & 0.97 & - \\
J0211.1-0646 & J021116.95-064419.9 & LEDA 1029376 & 0.99 & 0.96 \\
J0237.7+0206 & J023737.97+020742.5 & PKS 0235+017 & 0.93 & - \\
J0402.9+6433 & J040254.43+643510.0 & 1RXS J040301.8+643446 & 0.99 & 0.99 \\
J0640.9-5204 & J064111.26-520232.2 & ERC217 G261.25-22.62 & 0.99 & - \\
J0725.6-3530 & J072548.16-353041.8 & NVSS J072547-353039 & 0.61 & - \\
J0801.3-0617 & J080141.07-060535.2 & CRATES J080140-054037 & 0.93 & 0.92 \\
J0807.7-1206 & J080730.69-120608.9 & CRATES J080736.06-120745.9 & 0.96 & - \\
J1122.0-0231 & J112213.71-022914.0 & 2QZ J112156-0229 & 0.86 & - \\
J1136.3-0501 & J113547.40-050804.8 & NVSS J113607-050156 & 0.93 & 0.96 \\
J1145.7+0453 & J114631.75+045819.2 & PKS 1142+052 & 0.83 & 0.97 \\
J1300.4+1416 & J130020.92+141718.4 & OW 197 & 0.96 & - \\
J1305.3+5118 & J130645.87+511655.0 & IERS B1303+515 & 0.97 \\
J1516.8+2918 & J151641.71+291816.5 & RGB J1516+293 & 0.89 \\
J1518.6+0614 & J151847.70+061259.0 & TXS 1516+064 & 0.96 \\
J1550.8-1750 & J155053.18-175618.4 & TXS 1548-177 & 0.88 \\
J1734.0+0805 & J173510.44+080831.0 & 2MASS J17340287+0805237 & 0.86 & 0.83 \\
J1807.1+2822 & J180712.91+282059.5 & WISE J180634.08+281908.0 & 0.99 & - \\
J1953.0-7025 & J195306.71-702428.7 & PKS 1947-705 & 0.86 & 0.96 \\
J2110.2-1021 & J211038.30-103337.2 & PKS 2107-105 & 0.96 & 0.95 \\
J2209.8-5028 & J221040.80-502652.5 & PMN J2210-5030 & - \\
J2329.7-2118 & J232940.19-211345.0 & PKS 2327-215 & 0.97 & 0.88 \\
\hline
\end{tabular}

Table 7.1 - List of sources with associations provided with the LR method developed here and having a different association in 4FGL. As expected, most (but not all) of the associations listed in 4FGL are closer to the $\gamma$-ray emission center than the WIBRaLS candidate counterparts selected based on MIR colors. Starting from the left side, the columns are i) the name of the $\gamma$-ray source in 4FGL, ii) the WISE name of the WIBRaLS candidate counterpart selected based on MIR colors, iii) the candidate counterpart listed in 4FGL, iv) the AP obtained with the LR method developed here, and v) the AP obtained with the LR used in 4 FGL.

when considering angular separation are also similar. The total number of associated counterparts in this case is 580 with $\mathrm{AP}>99 \%$, or 1071 when considering $\mathrm{AP}>95 \%$. Furthermore, all sources with AP $<95 \%$ have low $\gamma$-ray statistical significance $(<10 \sigma)$, which generally implies in large positional and $\gamma$-ray photon index errors.

In the upper panel of Figure 7.5 we compare the APs obtained with the LR method developed here with those obtained with the LR adopted in 4FGL. In the bottom panel we show the same comparison, but when taking into account the angular separation, $r$, in the LR method. In both cases our method tend to give higher APs.

\subsection{Results from the random forest approach}

We found a total of 960 associations with $\mathrm{AP}>70 \%$ (i.e., in the same range of the identified 4FGL sources. See $\S 7.5$ ) out of 1311 test sources (see $\S 7.3$ ). Furthermore, the number of counterparts with AP $>50 \%$ increases to 1109 . Due to the low accuracy 


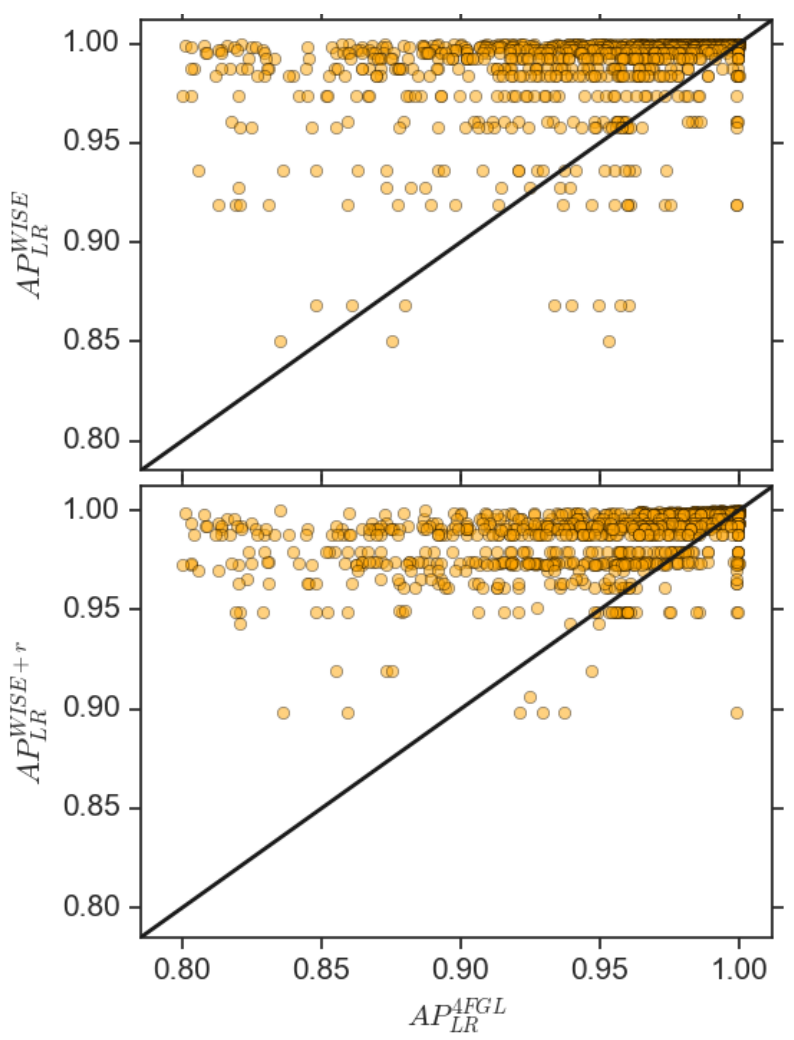

Figure 7.5: Comparison between the APs obtained with our LR method neglecting (upper panel) and considering (bottom panel) angular separation and the APs obtained with the LR method used in 4FGL. The APs computed by our method tend to be higher than those listed in 4FGL paper.

presented by the RF method (see $\S 7.5$ ), we use these results only as a comparison to the LR method described in the previous section. The APs for the BCUs and UGSs associated with the RF are shown in Tables 7.2 and 7.3 in Appendix 7.9.

When comparing the APs obtained with the RF algorithm and those obtained with the LR methods used here and in 4FGL, we found no significant correlation, although the APs computed with the RF approach tend to be smaller and distributed over a larger range. The major difference between both methods is the way we feed the two algorithms with the data. In Figure 7.1 it is clear that several fake associations (orange dots) occupy the same region as the high confidence associations, thus counterparts lying within the high confidence association region (black contours) still have a high chance of being a fake association in the RF approach. The same does not happen with the LR method. Furthermore, based on how we defined the training set and the fake associations, we are solving an intrinsically more complicated task with the RF approach than with the LR method. 


\subsection{Discussion and conclusions}

Associating $\gamma$-ray sources with their low-energy counterparts is challenging (Massaro et al., 2013). Given the absence of multiwavelength monitoring and optical spectroscopic data within the error ellipses of many 4FGL sources, we have to recur to statistical methods when associating $\gamma$-ray sources to their counterparts.

In this work we propose an alternative to the LR association method adopted by the Fermi-LAT Collaboration (Abdollahi et al., 2020). Here, the APs are independent of angular separation and apparent magnitude, and rely only on spectral parameters such as MIR colors and radio-loudness. As a complementary association method, we trained a RF algorithm to associate $\gamma$-ray sources to their counterparts. Both methods, however, are suited only for associating blazar candidates to $\gamma$-ray sources. Other types of $\gamma$-ray sources, like pulsars and starburst galaxies (to name a few), can not be directly associated with the proposed methods.

In the LR method adopted here we naturally loose some true blazar associations which have MIR colors just outside the blazar locus (i.e., the region of the WISE color-color-color diagram populated by confirmed $\gamma$-ray blazars). The sources associated here, however, are very good $\gamma$-ray blazar candidates and excellent targets for future optical spectroscopic follow ups. Furthermore, as all sources in WIBRaLS are radio-loud with respect to the $q_{22}$ parameter (D'Abrusco et al., 2019), it is likely to find many BL Lac-galaxy dominated among the sources excluded by our method. It is really unlikely that these sources have no sign of nuclear activity, as the presence of non-AGNs in WIBRaLS is estimated to be $<5 \%$ (de Menezes et al., 2019). The results from our analysis are as follows.

- The total number of associations computed with the LR method is 743 with AP $>99 \%$, or 1051 associations with AP $>95 \%$. We associate a total of 283 BCUs and 5 UGSs (listed in Tables 7.2 and 7.3 in Appendix 7.9).

- Adding a dependence on angular separation to the LR method do not change significantly the results, indicating that WIBRaLS sources are generally in good positional agreement with 4FGL sources.

- A supervised learning method is used for the first time to associate counterparts to $\gamma$ ray sources. As the performance of the algorithm is $\sim 80 \%$, the results derived from 
this method are considered complementary to those obtained with the LR method and should not be interpreted as giving definitive associations to 4FGL sources.

This is the first work where spectral properties of blazars are used to associate $\gamma$-ray sources to their low-energy counterparts. Previous methods rely basically on parameters like apparent magnitude and angular separation between the $\gamma$-ray source center and the position of its candidate counterpart.

The lists of BCUs and UGSs available in Appendix 7.9 give an excellent opportunity for testing the LR method presented here. In tables 7.2 and 7.3, the targets are sorted alphabetically and can be prioritized by choosing those with higher APs. Optical spectroscopy follow ups of these targets may be crucial to test if the association based on MIR colors is indeed an appropriate approach.

\section{Acknowledgements}

This work was supported by FAPESP (Fundação de Amparo à Pesquisa do Estado de São Paulo) under grants 2016/25484-9, 2018/24801-6 (R.M.) and 2017/01461-2 (R.N.). The work of F.M. is partially supported by the "Departments of Excellence 2018-2022" Grant awarded by the Italian Ministry of Education, University and Research (MIUR) (L. 232/2016) and made use of resources provided by the Compagnia di San Paolo for the grant awarded on the BLENV project (S1618_L1_MASF_01) and by the Ministry of Education, Universities and Research for the grant MASF_FFABR_17_01. F.M. also acknowledges financial contribution from the agreement ASI-INAF n.2017-14-H.0 while A.P. the financial support from the Consorzio Interuniversitario per la Fisica Spaziale (CIFS) under the agreement related to the grant MASF_CONTR_FIN_18_02. R.D'A. is supported by NASA contract NAS8-03060 (Chandra X-ray Center). This investigation is supported by the National Aeronautics and Space Administration (NASA) grants GO6-17081X and GO920083X. We thank the anonymous referee for the constructive comments allowing us to improve the manuscript. The full version of the acknowledgements can be found in the original paper by de Menezes et al. (2020). 


\begin{tabular}{|l|l|r|r|r|r|}
\hline 4FGL name & \multicolumn{1}{|c|}{ WISE name } & LR WISE & RF & Bayesian & LR 4FGL \\
\hline J0001.6-4156 & J000132.74-415525.2 & 0.99 & 0.97 & 1.0 & 0.85 \\
J0008.0-3937 & J000809.17-394522.8 & 0.87 & 0.61 & 0.92 & 0.88 \\
J0010.8-2154 & J001053.64-215704.2 & 0.96 & 0.42 & 0.99 & 0.95 \\
& & {$[\ldots]$} & & & \\
J1238.1-4541 & J123806.03-454129.6** & 0.99 & 1.0 & 1.0 & 0.94 \\
J1239.4+0728 & J123924.58+073017.2* & 1.0 & 0.96 & 1.0 & 0.94 \\
& & {$[\ldots]$} & & & \\
\hline
\end{tabular}

Table 7.2 - BCUs associated by the LR method based only on spectral parameters. The BCUs listed here are good targets for future optical spectroscopic follow ups. In the first two columns we have the names of the $\gamma$-ray source and its WISE counterpart. The last four columns show the APs as given by the LR and RF methods in this work and by the Bayesian and LR methods used in 4FGL. Sources tagged with "*" and "**" are confirmed blazars, with optical spectra available in de Menezes et al. (2020) and Peña-Herazo et al. (2020, in prep), respectively. The full version of this table is available in the online material.

\begin{tabular}{|l|l|r|c|}
\hline \multicolumn{1}{|c|}{ 4FGL Name } & \multicolumn{1}{|c|}{ WISE name } & LR WISE & RF \\
\hline J0202.4+2943 & J020239.70+294325.0 & 0.99 & 0.75 \\
J0620.7-5034 & J062046.13-503350.9 & 0.97 & 0.68 \\
J1028.1-3550 & J102751.56-355210.7 & 0.98 & 0.31 \\
J1309.3+8035 & J130628.16+803142.3 & 0.93 & 0.61 \\
J2013.9-8717 & J201440.97-871707.8 & 0.98 & 0.9 \\
\hline
\end{tabular}

Table 7.3 - UGSs associated by the LR method. These sources are good targets for future optical spectroscopic follow ups. In the first two columns we have the names of the $\gamma$-ray source and its WISE counterpart. The last two columns show the AP as given by the LR and RF methods.

\subsection{Appendix: List of targets for optical spectroscopic follow up}

A list with all BCUs associated with the LR method is provided in Table 7.2, where the sources are promising targets for future optical spectroscopic follow ups (Massaro et al., 2015; Ricci et al., 2015; Crespo et al., 2016). The columns are the name of the source in $\gamma$-rays and MIR, the AP obtained with the LR method developed here, the AP obtained with the RF approach, the AP obtained with the Bayesian method used in 4FGL and the AP computed with the LR method based on angular distance as in 4FGL. Similarly, Table 7.3 lists the 5 UGSs described in $\S 7.6$ and $\S 7.7$ and their APs. 
Chapter 8

\section{Spectroscopic follow-up of BCUs}

This thesis chapter is a shortened version of the paper "Optical spectroscopic observations of gamma-ray blazar candidates. X. Results from the 2018-2019 SOAR and OAN-SPM observations of blazar candidates of uncertain type" by de Menezes et al. (2020), published in the Astrophysical Journal Supplement Series, volume 248, number 2. The author was responsible for writing the paper, for half of the spectroscopic observations and for all of the data reduction. The original work can be found online here.

\section{Abstract}

The fourth Fermi Large Area Telescope Source Catalog (4FGL) lists over $5000 \gamma$ ray sources with statistical significance above $4 \sigma$. About $23 \%$ of the sources listed in this catalog are unidentified/unassociated $\gamma$-ray sources while $\sim 26 \%$ of the sources are classified as blazar candidates of uncertain type (BCUs), lacking optical spectroscopic information. To probe the blazar nature of candidate counterparts of UGSs and BCUs, we started our optical spectroscopic follow up campaign in 2012, which up to date account for more than 350 observed sources. In this paper, the tenth of our campaign, we report on the spectroscopic observations of 37 sources, mostly BCUs, whose observations were carried out predominantly at the Observatorio Astronómico Nacional San Pedro Mártir and the Southern Astrophysical Research Observatory between August 2018 and September 2019. We confirm the BL Lac nature of 27 sources and the flat spectrum radio quasar nature of three sources. The remaining ones are classified as six BL Lacs galaxy-dominated and one normal galaxy. We were also able to measure the redshifts for 20 sources, including $10 \mathrm{BL}$ 
Lacs. As in previous analyses, the largest fraction of BCUs revealed to be BL Lac objects.

\subsection{Introduction}

Blazars are one of the most peculiar and among the rarest class (Abdo et al., 2010) of active galactic nuclei (AGNs) whose emission, over the whole electromagnetic spectrum, is mainly arising from relativistic particles accelerated in a jet closely aligned to the line of sight (Blandford et al., 1978; Urry and Padovani, 1995). Together with radio galaxies they constitute the main class of AGNs emitting at MeV-to-TeV energies, with a few exceptions due to a small subset of nearby galaxies (Abdollahi et al., 2020).

There are two main sub-classes of blazars, distinguished on the basis of their optical spectra: BL Lac objects and flat spectrum radio quasars. The former subclass presents weak emission/absorption lines in their optical spectra with equivalent width (EW) smaller than $5 \AA$ (Stickel et al., 1991) or even completely featureless spectra, while sources belonging to the latter show quasar-like optical spectra with broad emission lines.

In the last decade we initiated and carried out an optical spectroscopic campaign to unveil the nature of potential counterparts of unidentified/unassociated $\gamma$-ray sources (UGSs, D'Abrusco et al., 2013; Massaro et al., 2016) discovered with the Fermi Large Area Telescope (LAT) and to confirm the classification of blazar candidates of uncertain type (BCUs; Ackermann et al., 2015).

Given the continuously increasing number of discovered UGSs by Fermi-LAT, our observations are mainly focused on searching blazar-like sources within the positional uncertainty of the UGSs (Acero et al., 2013; Massaro et al., 2015; Paggi et al., 2014) and/or eventually BCUs (Massaro et al., 2012). Given the large positional uncertainty of $\gamma$-ray sources, of the order of $\sim 4$ arcmin in the third and fourth releases of the Fermi-LAT point source catalogs (3FGL and 4FGL, Acero et al., 2015; Abdollahi et al., 2020), potential counterparts, targets of our spectroscopic observations, were selected on the basis of several criteria based, for example, on their mid-infrared colors (MIR; Massaro et al., 2011a; D'Abrusco et al., 2012), presence of X-ray emission (Paggi et al., 2013) and/or presence of radio sources (Schinzel et al., 2015).

Here, in the tenth paper of a series devoted to our optical spectroscopic campaign, we focused on the observations of a selected sample of BCUs, that being already associated 
with Fermi-LAT sources, needed an optical spectroscopic classification (Ackermann et al., 2015) to confirm their nature. In the present analysis, for the blazar classification, we adopted the nomenclature of the Roma-BZCAT (Massaro et al., 2015) distinguishing between BL Lac objects (i.e., BZBs), flat spectrum radio quasars (labelled as BZQs), as well as blazars of galaxy type (BZGs) whereas the spectrum is dominated by the emission from its host galaxy but presents a non-thermal blue continuum likely originated in a relativistic jet (see Section 8.3).

The main goal of our optical spectroscopic campaign is to "hunt" blazars among UGSs and BCUs, since they constitute the largest known population of $\gamma$-ray emitters (Abdollahi et al., 2020). If we confirm a blazar lies within the positional uncertainty region of a UGS, it bolsters its likelihood of being the counterpart of the UGS, due to the scarcity of such sources in the sky. It is thus a valuable information, that has been so far used in every subsequent release of the Fermi-LAT catalogs (Massaro et al., 2015). Even though there has been continued efforts to develop new and alternative methods to increase the association probability (e.g., D'Abrusco et al., 2019), still optical spectroscopy remains an efficient method that provides a precise classification of the potential counterpart of a $\gamma$-ray source.

The state of the art of our campaign can be briefly summarized as follows. We collected spectra for 337 sources to date and classified them as 255 BZBs, 39 BZQs and 20 BZGs, while additional 23 sources have been classified as normal quasars, due to the lack of multifrequency observations, particularly in the radio band, that could confirm the presence of a jet. In particular, 167 sources out of 337 were all BCUs for which the largest fraction (i.e., 114 out of 167, 70\%) were identified as BZBs, confirming that they are the most elusive subclass of blazars. During our campaign we extensively and continuously searched in the optical databases to exclude targets for which an optical spectrum was already available (see e.g., Massaro et al., 2016b) and we also compared our results with those present in the literature, due to other spectroscopic campaigns (Landoni et al., 2018; Paiano et al., 2019), to maintain an updated database. The largest fraction of the sources classified to date come from the archival search in Sloan Digital Sky Survey (SDSS) data, which accounts for a total of 127 spectra; and then more than 100 observations were also carried out in the southern hemisphere with the Southern Astrophysical Research Telescope (SOAR) telescope. All remaining ones were performed with other $2 \mathrm{~m}$ - to 6 
m-class telescopes as the Kitt Peak National Observatory (KPNO), the William Herschel Telescope (WHT), Telescopio Nazionale Galileo (TNG), Nordic Optical Telescope (NOT), Observatorio Astrofísico Guillermo Haro (OAGH), Observatorio Astronómico Nacional San Pedro Mártir (OAN-SPM) and Multiple Mirror Telescope (MMT) to name a few.

Our spectroscopic identifications were used by other groups to (i) build the luminosity function of BL Lacs (Ajello et al., 2013); (ii) select potential targets for the Cherenkov Telescope Array (CTA; Arsioli et al., 2015); (iii) obtain stringent limits on the dark matter annihilation in sub-halos (e.g., Berlin et al., 2014); (iv) search for counterparts of new flaring $\gamma$-ray sources (Bernieri et al., 2013); (v) test new $\gamma$-ray detection algorithms (Campana et al., 2016); (vi) perform population studies on the UGSs (e.g., Acero et al., 2013) and (vii) discover the new subclass of radio weak BL Lacs (e.g., Massaro et al., 2017). All the above studies confirm the legacy value of our results thus motivating the prosecution of our BCU follow up campaign.

The present paper is organized as follows. In $\S 8.2$ we present the selection criteria for the the BCU sample analysed here, while in $\S 8.3$ the data reduction procedures for SOAR and OAN-SPM observations are described. In $\S 8.4$ we discuss results obtained, and $\S 8.5$ is devoted to our summary and conclusions.

\subsection{Sample selection}

Our sample consists of 37 sources predominantly listed as counterparts of $\gamma$-ray sources in the 4FGL catalog (35 sources), as well as in the Fermi-LAT 8-year Point Source List ${ }^{1}$ (FL8Y; 2 sources), a preliminary release of 4FGL based on the same 8-years of data. We highlight that the two sources selected from FL8Y do not appear in 4FGL. The 37 targets are listed as 33 BCUs and 4 BZBs in these $\gamma$-ray catalogs.

Sources classified as BCUs have unknown blazar nature, as all BCUs i) lack an optical spectra sensitive enough to classify them as BZBs or BZQs, or ii) are classified as blazars of uncertain or transitional types in Roma-BZCAT. However, all BCUs have multiwavelength data indicating a typical blazar-like behaviour (see Ackermann et al., 2015; Abdollahi et al., 2020) and thus, as previously stated, our spectroscopic analysis has been carried out to confirm their real nature.

\footnotetext{
${ }^{1}$ https://fermi.gsfc.nasa.gov/ssc/data/access/lat/fl8y/
} 
In 4FGL, there are 1312 associated sources classified as BCUs (i.e., 26\% of the entire catalog), a fraction significantly higher than on its predecessor 3FGL, for which the BCUs accounted for $19 \%$ of the sample (Acero et al., 2015). All targets selected in our sample have optical magnitude in the $r$ band brighter than 18.5, as listed in the USNO-B catalog (Monet et al., 2003). During our observing nights we were able to observe 33 BCUs and 4 sources already classified as BZBs for which we searched for a spectroscopic confirmation and potentially a redshift estimate.

We also verified which of our targets are listed in the latest releases of the Wide-field Infrared Survey Explorer (WISE) Blazar-Like Radio-Loud Sources catalog (WIBRaLS; D'Abrusco et al., 2019) and the Kernel Density Estimation selected candidate BL Lacs catalog (KDEBLLACS; D'Abrusco et al., 2019), both known to present a high number of spectroscopically confirmed blazars (de Menezes et al., 2019). We found a total of 19 matches with WIBRaLS (14 classified here as BZBs, 2 BZGs and 3 BZQs; see $§$ 8.4) and 3 more matches with KDEBLLACS (all classified here as BZBs, see $\S$ 8.4). Additionally, two of our targets, 4FGL J1208.4+6121 and 4FGL J1239.4+0728, are listed in Roma-BZCAT and classified there as blazars of uncertain type.

Although we selected the BCUs mainly from 4FGL, in our previous papers we tend to select sources based on MIR methods, as the Fermi-LAT $\gamma$-ray blazars are known to occupy a distinct area in the WISE MIR color-color-color diagram (D'Abrusco et al., 2012, 2019). Here we compare the distribution of our targets in the WISE MIR color-color diagrams W1-W2 vs. W2-W3 and W2-W3 vs. W3-W4, using the magnitudes measured at the four WISE bands W1[3.4 $\mu \mathrm{m}], \mathrm{W} 2[4.6 \mu \mathrm{m}], \mathrm{W} 3[12 \mu \mathrm{m}]$ and W4[22 $\mu \mathrm{m}]$, with the distribution of MIR colors for $\gamma$-ray blazars listed in Roma-BZCAT, finding that all of them have MIR colors consistent with $\gamma$-ray blazars (Figure 8.1), except perhaps for 4FGL J1612.2+2828/WISE J161217.62+282546.3, the black star in the bottom of both panels, classified as the only normal galaxy in our sample. Among our sources, 27 are detected in WISE W4 band and plotted as black stars in Figure 8.1. The remaining targets are plotted as white stars. The distribution of our targets in these diagrams suggest that their infrared emission is dominated by non-thermal radiation, as expected for $\gamma$-ray blazars.

WISE magnitudes used here are in the Vega system and are not corrected for the Galactic extinction. As shown in our previous analyses (D'Abrusco et al., 2013), such correction affect only the magnitude measures at $3.4 \mu \mathrm{m}$ for sources lying at low Galactic 

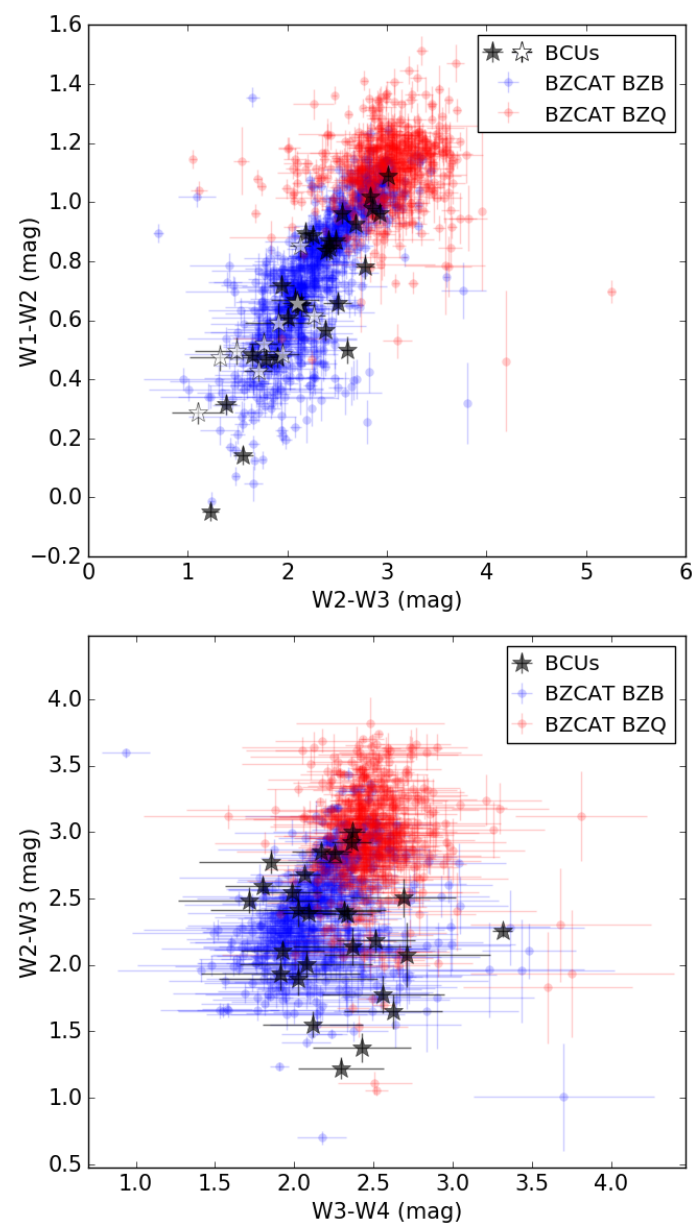

Figure 8.1: WISE color-color diagrams. Top: distribution of Roma-BZCAT $\gamma$-ray BZBs (blue circles), BZQs (red circles) and our targets (stars) in the W1-W2 $\times$ W2-W3 diagram. Here, black stars represent the targets detected in WISE W4 band, while the white stars lack such a detection. Bottom: same as upper panel but for $\mathrm{W} 2-\mathrm{W} 3 \times \mathrm{W} 3-\mathrm{W} 4$.

latitudes, and it ranges between $2 \%$ and $5 \%$, thus being almost negligible.

\subsection{Observations and data reduction}

We observed a total of 25 targets with the $2.1 \mathrm{~m}$ telescope at OAN-SPM, in Baja California, México, during several nights in 2018-2019 (see Table 8.1). The data were collected with the Boller \& Chivens spectrograph, with a slit width of $2.5^{\prime \prime}$, grating of 300 l/mm, wavelength range from $3800 \AA$ up to $7800 \AA$, and resolution of $\sim 14 \AA$.

A single source, 4FGL J0434.7+0922/TXS 0431+092, was observed with the $2.1 \mathrm{~m}$ telescope at OAGH in Cananea, México, using the Boller \& Chivens spectrograph. The collected spectrum has a wavelength range from $\sim 3600$ to $7300 \AA$ and was acquired with a slit width of $2.5^{\prime \prime}$ and grating of $150 \mathrm{l} / \mathrm{mm}$, having a final resolution of $\sim 14 \AA$. 


\begin{tabular}{|c|c|}
\hline & 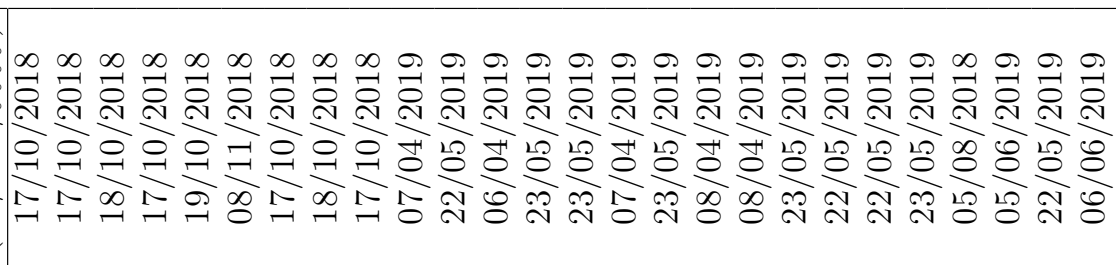 \\
\hline & 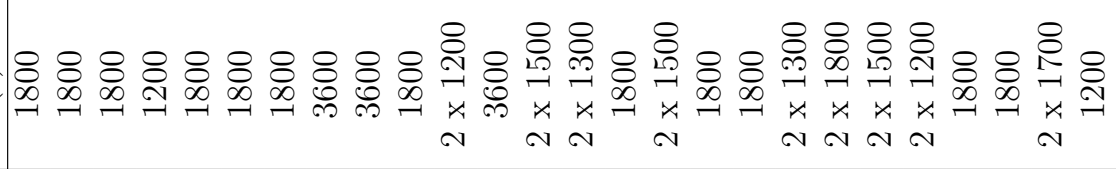 \\
\hline & \\
\hline & 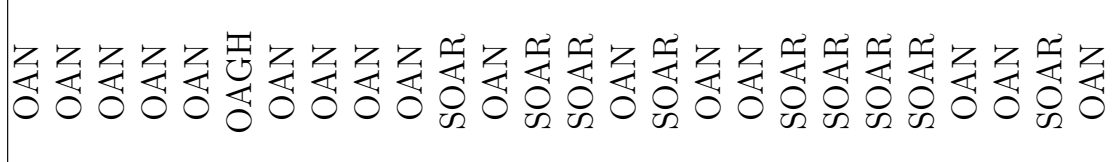 \\
\hline & 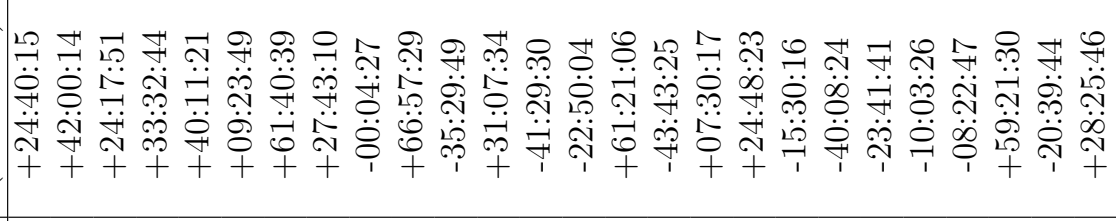 \\
\hline & 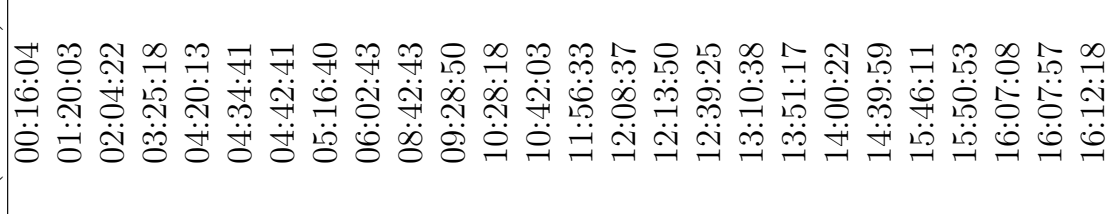 \\
\hline 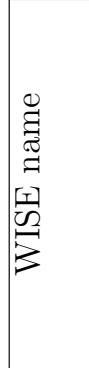 & 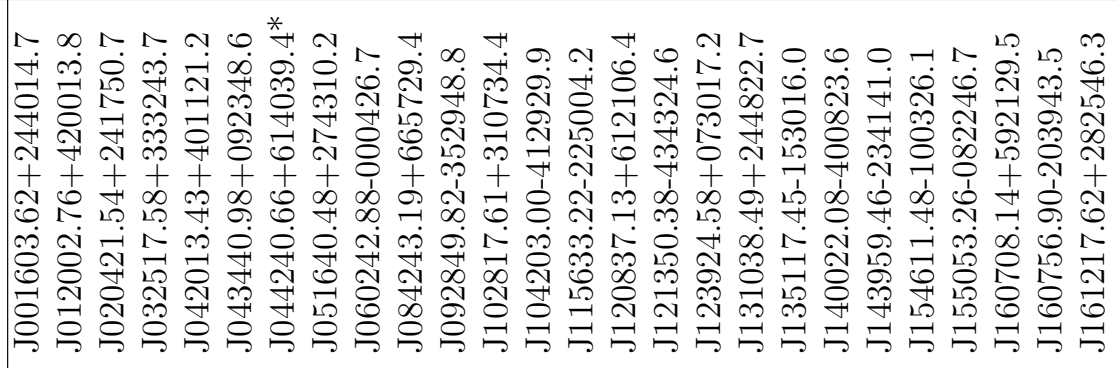 \\
\hline & 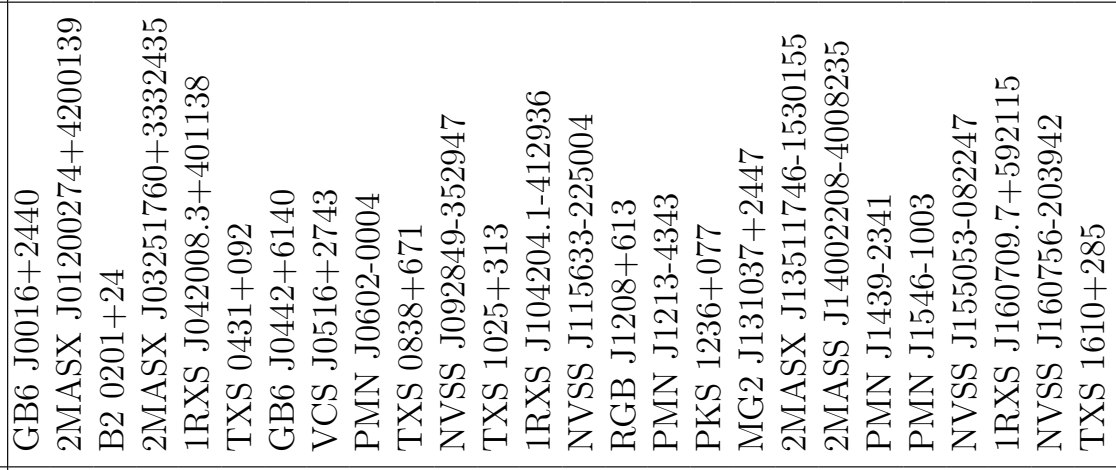 \\
\hline$\underset{\widetilde{Z}}{\mathbb{Z}}$ & 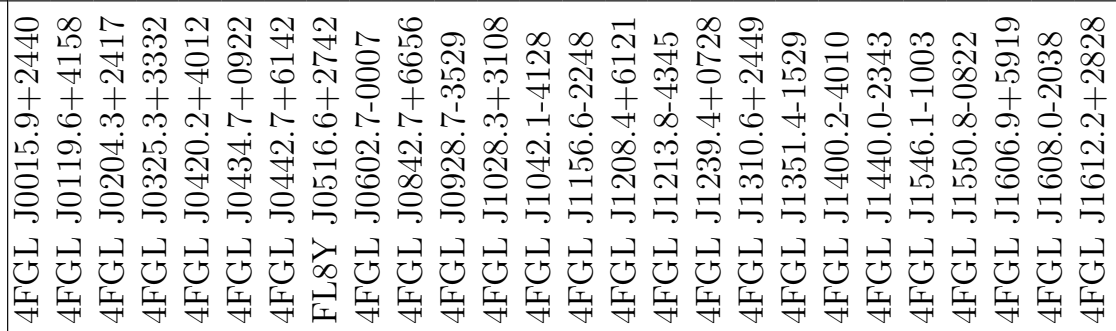 \\
\hline
\end{tabular}

푱

过 $\cong$

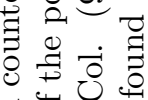
荧 虽 \&

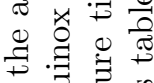

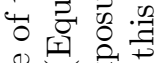

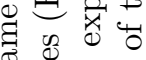

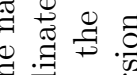

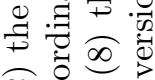
กิ 8 ن் O

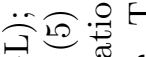
च ఫ్ష 索声 I -5 है $=0$ क $\stackrel{-10}{\circ}$ 60 员 票罚 ○ 更六 䛼氖思 . क्षे \&

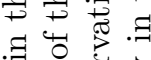

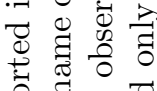

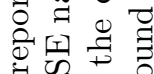

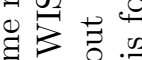
寻

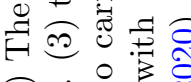

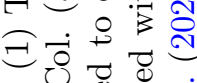
ن 000 .

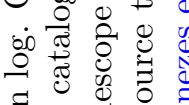

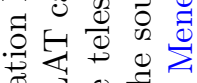

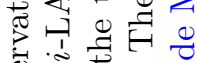
हैं 1 . के

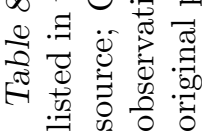


The data for the remaining 11 targets were collected with the $4.1 \mathrm{~m}$ SOAR telescope at Cerro Pachón, Chile, in remote observing mode on May 22 and 23 of 2019. We used the single, long slit mode of the Goodman High Throughput Spectrograph (Clemens et al., 2004) with slit width of $1^{\prime \prime}$ and a grating of $400 \mathrm{l} / \mathrm{mm}$, giving a dispersion of $\sim 3 \AA$ per pixel in a spectral range from $\sim 4100 \AA$ up to $7900 \AA$, and resolution of $\sim 6 \AA$.

In Table 8.1 we report the log of all observations, giving the name of the $\gamma$-ray source and its associated counterpart in the Fermi-LAT catalogs, the associated counterpart in WISE, the telescope used, the average signal to noise ratio (SNR), the exposure time and the date of observation.

The data were reduced using standard procedures, with the images trimmed, bias subtracted, corrected for flat field and stacked using standard IRAF packages (Tody, 1986). The cosmic ray removal was performed with Python packages ccdproc (Craig et al., 2015) and astropy (Price-Whelan et al., 2018), in the case of SOAR data, and using the L.A. Cosmic IRAF algorithm (Van Dokkum 2001) in the case of OAN-SPM data. All spectra were then flux and wavelength calibrated using, respectively, a standard spectrophotometric star and a Hg-Ar (SOAR) or CuNeHeAr (OAN-SPM) lamp. The corrections for Galactic extinction were made using the reddening law of Cardelli et al. (1989) and values of visual extinction $A_{v}$ computed based on Schlafly and Finkbeiner (2011) assuming a visual extinction to reddening ratio $A_{v} / E(B-V)=3.1$. Finally, we performed a box smoothing for visual representation, normalized the spectra using a 5th degree polynomial fitted to the continuum to highlight possible spectroscopic features, and visually identified the emission/absorption lines (Figure 8.2).

\subsection{Analysis and results}

Thanks to the new optical spectra collected, we are able to confirm the blazar nature for 36 targets in our sample, consisting of 27 BZBs, 6 BZGs, and 3 BZQs. Additionally, we identified a normal galaxy listed in 4FGL as the counterpart of 4FGL J1612.2+2828.

We obtained the redshifts $(z)$ for 20 of the targets, including 10 BZBs, all with relatively low $z$ distributed in the range 0.12-0.28, as expected for BZBs. Particularly we successfully measured redshifts for 3 of the 4 BZBs listed in the 4FGL (see Section 8.2). They are 4FGL J0420.2+4012/WISE J042013.43+401121.2 with $\mathrm{z}=0.132$, 4FGL 

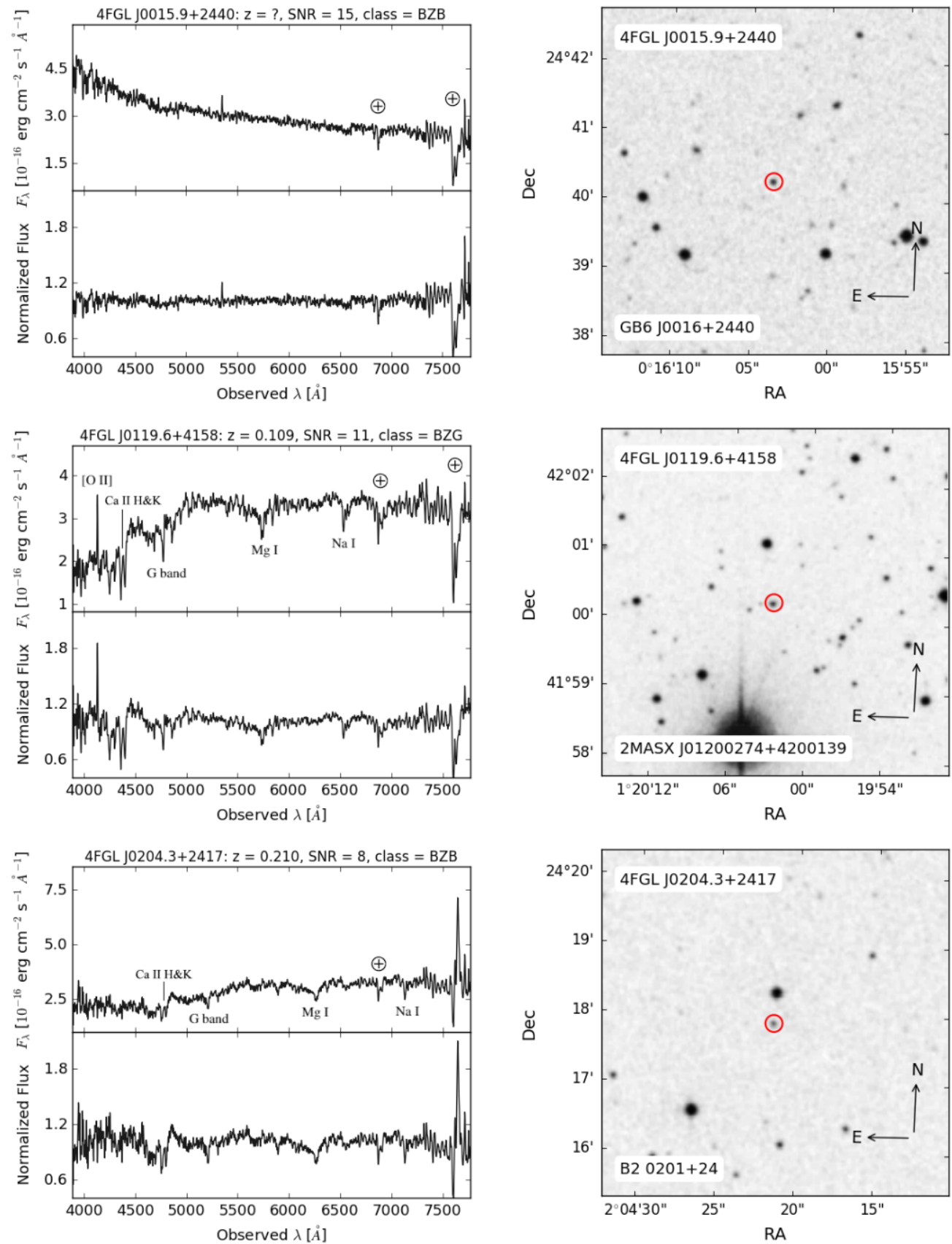

Figure 8.2: Left: flux calibrated and normalized optical spectra. Right: finding charts. The name of the $\gamma$-ray sources and their respective counterparts listed in the Fermi-LAT catalogs are indicated, together with the spectral class and average SNR. Redshifts and emission/absorption lines are shown whenever possible. The full version of this figure can be found in the original paper by de Menezes et al. (2020). 


\begin{tabular}{|c|c|c|c|c|c|c|c|c|}
\hline Fermi-LAT name & WISE counterpart & Class & $\mathrm{C}$ & $z$ & Line & EW $(\AA)$ & $\lambda_{o b s}(\AA)$ & Type \\
\hline 4FGL J0015.9+2440 & J001603.62+244014.7 & BZB & & & & & & \\
\hline \multirow[t]{5}{*}{ 4FGL J0119.6+4158 } & J012002.76+420013.8 & BZG & 0.30 & 0.109 & {$[\mathrm{O} \quad \mathrm{II}]$} & 5 & 4132 & $\mathrm{E}$ \\
\hline & & & & & Ca II H\&K & $10 ; 9$ & $4362 ; 4399$ & A \\
\hline & & & & & G band & & 4773 & A \\
\hline & & & & & Mg I & & 5737 & A \\
\hline & & & & & & & 6532 & A \\
\hline \multirow[t]{4}{*}{ 4FGL J0204.3+2417 } & J020421.54+241750.7 & BZB & 0.21 & 0.210 & Ca II H\&K & $7 ; 5$ & $4760 ; 4803$ & A \\
\hline & & & & & G band & 3 & 5510 & A \\
\hline & & & & & Mg I & 5 & 6267 & A \\
\hline & & & & & $\mathrm{Na} \mathrm{I}$ & 4 & 7131 & A \\
\hline \multirow[t]{3}{*}{ 4FGL J0325.3+3332 } & J032517.58+333243.7 & BZG & 0.36 & 0.128 & $\mathrm{Ca}$ II H\&K & $12 ; 9$ & $4440 ; 4477$ & A \\
\hline & & & & & $\mathrm{G}$ band & 6 & 4855 & A \\
\hline & & & & & Mg I & 6 & 5838 & A \\
\hline \multirow[t]{3}{*}{ 4FGL J0420.2+4012 } & J042013.43+401121.2 & BZB & 0.01 & 0.132 & $\mathrm{G}$ band & 4 & 4874 & A \\
\hline & & & & & Mg I & 6 & 5860 & A \\
\hline & & & & & $\mathrm{Na} I$ & 4 & 6676 & A \\
\hline 4FGL J0434.7+0922 & J043440.98+092348.6 & BZB & & & & & & \\
\hline 4FGL J0442.7+6142 & J044240.66+614039.4 & $\mathrm{BZB}$ & & & & & & \\
\hline \multirow[t]{4}{*}{ FL8Y J0516.6+2742 } & J051640.48+274310.2 & BZG & 0.34 & 0.060 & [O II] & 5 & 3952 & $\mathrm{E}$ \\
\hline & & & & & Ca II H\&K & $8 ; 6$ & $4172 ; 4206$ & A \\
\hline & & & & & G band & 3 & 4566 & A \\
\hline & & & & & Mg I & 5 & 5492 & A \\
\hline
\end{tabular}

Table 8.2 - Summary of our spectroscopic identifications. Col. (1) the name reported in the Fermi-LAT catalogs (i.e., FL8Y and 4FGL); Col. (2) the WISE name of the associated counterpart; Col. (3) the source class (i.e., BZB for BL Lac objects, BZQ for Blazars of quasar type, BZG for blazars of galaxy type); Col. (4) the measured Ca II H\&K contrast; Col. (5) measured redshift; Col. (6) Emission or Absorption lines identified; Col. (7) Measured equivalent width (EW); Col. (8) Observed wavelength of each emission/absorption line identified; Col. (9) type of line: absorption (A) or emission (E). The full version of this table can be found in the original paper by de Menezes et al. (2020).

J2220.5+2813/WISE J222028.72+281355.8 with $\mathrm{z}=0.148$ and 4FGL J2358.3+3830/WISE $\mathrm{J} 235825.19+382856.5$ with $\mathrm{z}=0.200$. For the last source, we observed emission lines with $\mathrm{EW}>5 \AA$, and thus classified it as a BZQ.

All results achieved for the entire sample are summarized in Table 8.2, where we show the name of the $\gamma$-ray sources as listed in the Fermi-LAT catalogs, the name of the WISE counterparts, the redshifts when available, the spectral classes and information about the observed absorption/emission lines.

The optical spectra and finding charts for the analysed sources are available in Figure 8.2, ordered by right ascension. Finding charts were obtained from ESO Online Digitized Sky Survey ${ }^{2}$ and have a size of $5^{\prime} \times 5^{\prime}$. The name of each emission/absorption line is also indicated in the figure. Telluric absorption lines are labelled with $\bigoplus$.

Criteria adopted to distinguishing between BZBs, BZGs and normal galaxies are based

\footnotetext{
2 http://archive.eso.org/dss/dss
} 
on the observed Ca II H\&K break contrast. We computed the ratio $C=\left(F_{+}-F_{-}\right) / F_{+}$ where $F_{+}$and $F_{-}$are the mean flux densities measured in small ranges of $200 \AA$ just above

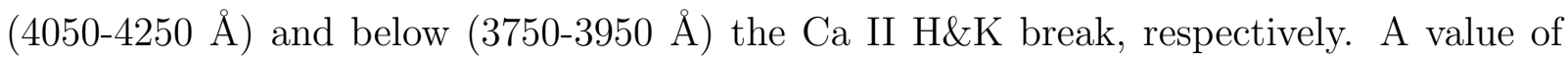
$C<0.4$ means that the source has a blue continuum emission dominated by non-thermal radiation likely originated in a relativistic jet (Landt et al., 2002). Then sources with $C>0.4$ are classified as normal galaxies, sources with $0.25<C \leq 0.4$ are classified as BZG, while values of $C \leq 0.25$ lead towards a BZB or BZQ classification.

To distinguish between BZBs and BZQs we computed the rest frame equivalent width (EW) of their emission lines (when available) and classified them as BZBs if all lines had $\mathrm{EW} \leq 5 \AA$. In particular, we found only 3 BCUs having emission lines with $\mathrm{EW}>5 \AA$, leading to a BZQ classification.

For those sources for which a redshift estimate has been provided, the redshift uncertainties are of the order of $\Delta \mathrm{z}=0.001$ for the data collected with SOAR, while those pointed with OAGH and OAN-SPM have $\Delta z \sim 0.004$.

\subsection{Summary and Conclusions}

We presented the tenth paper of our optical spectroscopic campaign aiming at the identification of BCUs listed in Fermi-LAT catalogs. We collected and analysed data for a total of 37 sources, confirming the blazar nature for 36 of them. Our results are summarized as follows:

- Among the 33 sources classified as BCUs in the Fermi-LAT catalogs, 24 of them $(73 \%)$ are found to be BZBs, 6 are BZGs (18\%), 2 are BZQs (6\%) and 1 is a normal galaxy (3\%). We also measured the redshifts for 17 of them.

- We observed 4 sources classified as BZBs in 4FGL and were able to measure the redshifts for 3 of them: 4FGL J0420.2+4012, 4FGL J2220.5+2813 and 4FGL J2358.3 +3830 , with $\mathrm{z}=0.132, \mathrm{z}=0.148$ and $\mathrm{z}=0.200$ respectively. In particular, 4FGL J2358.3+3830 presents emission lines with EW $>5 \AA$, thus being classified as BZQ.

- All sources classified as blazars (36 out of 37) have MIR colors consistent with what is expected for $\gamma$-ray blazars (Figure 8.1), except perhaps for the counterpart of 4FGL J1608.0-2038. 
The results here, as in the previous papers of our campaign (see e.g., Marchesini et al., 2019; Peña-Herazo et al., 2019), confirm that the largest fraction of BCUs tend to be BZBs. These objects, although very common in the $\gamma$-ray sky, continue to be one of the most elusive classes of $\gamma$-ray sources.

Our optical spectroscopic campaign is still ongoing with observing nights scheduled for the current and next semesters. New results will be made available as soon as they are collected and analysed. The next paper of the campaign will focus mainly on UGSs (Peña-Herazo et al. 2020) observed with the $4 \mathrm{~m}$ Blanco telescope at Cerro Tololo, Chile. We observed a total of 374 targets to date, mostly BCUs and UGSs, but including a small fraction (i.e., of the order of a few percent) of known blazars, especially BL Lac objects, trying to catch them in a low optical state and obtain a $z$ estimate. Particularly, out of 200 sources previously classified as BCUs, we found that 141 of them are BZBs.

\section{Acknowledgements}

This work was supported by FAPESP (Fundação de Amparo à Pesquisa do Estado de São Paulo) under grants 2016/25484-9 and 2018/24801-6 (R.M.). R.A.A-A. and H.P. acknowledge support from the CONACyT program for Ph.D. studies. The work of F.M. and A.P. is partially supported by the "Departments of Excellence 2018-2022" Grant awarded by the Italian Ministry of Education, University and Research (MIUR) (L. 232/2016) and made use of resources provided by the Compagnia di San Paolo for the grant awarded on the BLENV project (S1618_L1_MASF_01) and by the Ministry of Education, Universities and Research for the grant MASF_FFABR_17_01. F.M. also acknowledges financial contribution from the agreement ASI-INAF n.2017-14-H.0 while A.P. the financial support from the Consorzio Interuniversitario per la Fisica Spaziale (CIFS) under the agreement related to the grant MASF_CONTR_FIN_18_02. F.R. acknowledges support from FONDECYT Postdoctorado 3180506 and CONICYT project Basal AFB-170002. C.C.C. at NRL was supported by the Chief of Naval Research. The full version of these acknowledgements can be found in the original paper by de Menezes et al. (2020). 
Chapter 9

\section{Conclusion and future directions}

Throughout this $\mathrm{PhD}$, our research encompassed several topics on high energy astrophysics. These topics were mostly related to the $\gamma$-ray emission from the surroundings of compact astrophysical objects such as neutron stars and black holes, allowing us to explore a variety of interesting phenomena, like the dynamical processes leading to MSP formation in globular clusters, the $\gamma$-ray emission from $\pi^{0}$ decay in hot accretion flows, and the $\gamma$-ray-infrared color connection for blazars.

The first topic we covered was on how the stellar encounter rate affects the stability of binary systems and hence the formation of MSPs within globular clusters (Chapter 4). This work was mainly based on Fermi-LAT observations in conjunction with archival optical-infrared data, and allowed us to characterize some dynamical aspects of globular clusters. We found that:

- For a sufficiently large encounter rate per formed binary $(\Lambda)$, the binary systems in a globular cluster will quickly be disrupted, thus preventing the formation of MSPs. This is probably the reason why some nearby globular clusters are not detectable by Fermi-LAT.

- The cluster's metallicity does not play a role on MSP formation. The hypothesis that an environment with higher metallicity enhances the effect of magnetic braking, hence promoting the shrinkage of binary systems and consequently the formation of MSPs, is commonly found on literature. However, our analysis does not support this scenario.

- None of the globular clusters presented extended $\gamma$-ray emission, which is consistent with heavier objects (like MSPs in binary systems) sinking to the core of the cluster 
via dynamical friction.

The next step on this topic is to explore the $\gamma$-ray emission from dwarf elliptical galaxies in the neighborhood of the Milky Way, and test if their high energy emission can be extrapolated from the results we acquired for globular clusters. Dwarf ellipticals have never been observed above $5 \sigma$ with Fermi-LAT, but they can be detected as weak (bellow the $5 \sigma$ detection threshold) $\gamma$-ray sources. Given that they are weak $\gamma$-ray emitters, a few more years of Fermi-LAT observations may be essential for any statistically significant result.

In the second topic of this thesis, we investigated how $\pi^{0}$ decay contributes to the observed $\gamma$-ray flux from LLAGNs (Chapter 5). This time we used more than 10 years of Fermi-LAT observations, archival radio to X-ray data, and SED modelling to find that:

- Among 200 LLAGNs analyzed, only four of them are detected by Fermi-LAT, and these are precisely the brightest radio sources in the sample, suggesting that the observed $\gamma$-ray emission is related to the relativistic jet, even in cases where the jet is clearly misaligned in respect to the line of sight.

- The shape of the LLAGN SEDs analyzed here do not support a significant $\gamma$-ray emission caused by $\pi^{0}$ decay. Instead, the SEDs are better (but not fully) described by a SSC model.

- The data shows a positive trending between $\gamma$-ray and radio emission in LLAGNs. If this trending holds in future works, this result disfavors magnetospheric gap models for $\gamma$-ray emission in LLAGNs, as in the magnetospheric gap scenario the $\gamma$-ray luminosity increases when the accretion flow (and thus radio power) decreases.

The results achieved on this work represent the first systematic study performed with Fermi-LAT data on LLAGNs as a population, and open a window to future projects as, for instance, a $\gamma$-ray stacking analysis on LLAGNs with no detectable radio jets, thus avoiding the $\gamma$-ray contamination from the jet in the possible $\pi^{0}$ induced $\gamma$-ray emission.

On the last topic of this thesis, we focused on the hunt and association of counterparts for Fermi-LAT $\gamma$-ray blazars by taking into account the $\gamma$-ray-MIR connection (Chapters 6, 7 and 8). We started by using SDSS archival optical spectra to find the percentage of blazars listed in two catalogs of infrared candidate counterparts for $\gamma$-ray blazars, namely 
WIBRaLS and KDEBLLACS. Both catalogs proved to be good in spotting the counterparts of $\gamma$-ray blazars, with WIBRaLS showing better results. We then used WIBRaLS on the development of two new methods of $\gamma$-ray counterpart association, one based on a likelihood ratio technique and the other on a machine learning approach. Both methods compute an association probability for a given counterpart candidate based on spectral parameters only, thus being completely independent of non-physical parameters (e.g., angular separation), which are extensively used in the literature of $\gamma$-ray counterpart association. To complete our quest on the hunt for counterparts, we performed optical spectroscopic observations of a few tens of Fermi-LAT BCUs, classifying them as BL Lacs or FSRQs and in some cases providing their redshifts. The main results we found are as follows:

- WIBRaLS is composed of at least $\sim 30 \%$ and at maximum $\sim 95 \%$ of blazars, while for KDEBLLACS this range goes from $\sim 30 \%$ up to $\sim 65 \%$.

- The interplay between optical and infrared observations allowed us to precisely classify several BCUs which are now listed as counterparts of Fermi-LAT blazars in 4FGL.

- We developed alternative methods for $\gamma$-ray counterpart association which, when taking 4FGL and WIBRaLS into account, resulted in a list with $\sim 100$ candidate BCU associations which are excellent targets for future optical spectroscopic follow ups.

Despite the results above, there is still plenty of room for working on this topic. The next logical step here is to perform optical spectroscopic observations of the $\sim 100$ BCUs selected by our new association method to test how efficient the method is in spotting true blazar candidates, and then quantitatively compare our method with other methods available on literature.

In this thesis we worked on three topics of high energy astrophysics, and each of these topics opened a window for new developments in this field. We hope our results can contribute to the community and especially with the upcoming science based on FermiLAT observations and future observatories such as CTA. 


\section{Bibliography}

Abdalla H., Abramowski A., Aharonian F., Benkhali F. A., Angüner E., Arakawa M., Armand C., Arrieta M., Backes M., Balzer A., et al., The $\gamma$-ray spectrum of the core of Centaurus A as observed with HESS and Fermi-LAT, A\&A, 2018, vol. 619, p. A71

Abdo et al., Gamma-ray emission concurrent with the nova in the symbiotic binary V407 Cygni, Science, 2010, vol. 329, p. 817

Abdo A., Ackermann M., Agudo I., Ajello M., Aller H., Aller M., Angelakis E., Arkharov A., Axelsson M., Bach U., et al., The spectral energy distribution of Fermi bright blazars, The Astrophysical Journal, 2010, vol. 716, p. 30

Abdo A., Ackermann M., Ajello M., Atwood W., Axelsson M., Baldini L., Ballet J., Barbiellini G., Baring M., Bastieri D., et al., The first Fermi Large Area Telescope catalog of gamma-ray pulsars, The Astrophysical Journal Supplement Series, 2010, vol. 187, p. 460

Abdo A., Ackermann M., Ajello M., Atwood W., Axelsson M., Baldini L., Ballet J., Barbiellini G., Bastieri D., Baughman B., et al., Detection of high-energy gamma-ray emission from the globular cluster 47 Tucanae with Fermi, science, 2009, vol. 325, p. 845

Abdo A., Ackermann M., Ajello M., Atwood W., Axelsson M., Baldini L., Ballet J., Barbiellini G., Bastieri D., Bechtol K., et al., Fermi Large Area Telescope gamma-ray detection of the radio galaxy M87, The Astrophysical Journal, 2009, vol. 707, p. 55

Abdo A., Ackermann M., Ajello M., Baldini L., Ballet J., Barbiellini G., Bastieri D., Bechtol K., Bellazzini R., Berenji B., et al., Fermi large area telescope observations of misaligned active galactic nuclei, The Astrophysical Journal, 2010, vol. 720, p. 912 
Abdo A. A., Ackermann M., Ajello M., Allafort A., Antolini E., Atwood W., Axelsson M., Baldini L., Ballet J., Barbiellini G., et al., Fermi large area telescope first source catalog, The Astrophysical Journal Supplement Series, 2010, vol. 188, p. 405

Abdo A. A., Ackermann M., Ajello M., Baldini L., Ballet J., Barbiellini G., Bastieri D., Bellazzini R., Blandford R. D., Bloom E. D., et al., A population of gamma-ray emitting globular clusters seen with the Fermi Large Area Telescope, Astronomy \& Astrophysics, 2010, vol. 524, p. A75

Abdollahi S., Acero F., Ackermann M., Ajello M., Atwood W., Axelsson M., Baldini L., Ballet J., Barbiellini G., Bastieri D., et al., Fermi Large Area Telescope Fourth Source Catalog, The Astrophysical Journal Supplement Series, 2020, vol. 247, p. 33

Acero F., Ackermann M., Ajello M., Albert A., Atwood W., Axelsson M., Baldini L., Ballet J., Barbiellini G., Bastieri D., et al., Fermi large area telescope third source catalog, The Astrophysical Journal Supplement Series, 2015, vol. 218, p. 23

Acero F., Donato D., Ojha R., Stevens J., Edwards P., Ferrara E., Blanchard J., Lovell J., Thompson D., Hunting for treasures among the Fermi unassociated sources: a multiwavelength approach, The Astrophysical Journal, 2013, vol. 779, p. 133

Ackermann M., Ajello M., Allafort A., Antolini E., Atwood W., Axelsson M., Baldini L., Ballet J., Barbiellini G., Bastieri D., et al., The second catalog of active galactic nuclei detected by the Fermi Large Area Telescope, The Astrophysical Journal, 2011, vol. 743, p. 171

Ackermann M., Ajello M., Allafort A., Antolini E., Baldini L., Ballet J., Barbiellini G., Bastieri D., Bechtol K., Bellazzini R., et al., Gamma-ray observations of the orion molecular clouds with the fermi large area telescope, The Astrophysical Journal, 2012, vol. 756, p. 4

Ackermann M., Ajello M., Allafort A., Baldini L., Ballet J., Barbiellini G., Baring M., Bastieri D., Bechtol K., Bellazzini R., et al., Detection of the characteristic pion-decay signature in supernova remnants, Science, 2013, vol. 339, p. 807

Ackermann M., Ajello M., Allafort A., Baldini L., Ballet J., Barbiellini G., Bastieri D., Bechtol K., Bellazzini R., Berenji B., et al., Search for gamma-ray emission from X-ray- 
selected Seyfert galaxies with Fermi-LAT, The Astrophysical Journal, 2012, vol. 747, p. 104

Ackermann M., Ajello M., Atwood W., Baldini L., Ballet J., Barbiellini G., Bastieri D., Gonzalez J. B., Bellazzini R., Bissaldi E., et al., The third catalog of active galactic nuclei detected by the Fermi Large Area Telescope, The Astrophysical Journal, 2015, vol. 810 , p. 14

Actis M., Agnetta G., Aharonian F., Akhperjanian A., Aleksić J., Aliu E., Allan D., Allekotte I., Antico F., Antonelli L., et al., Design concepts for the Cherenkov Telescope Array CTA: an advanced facility for ground-based high-energy gamma-ray astronomy, Experimental Astronomy, 2011, vol. 32, p. 193

Aguado D., Ahumada R., Almeida A., Anderson S. F., Andrews B. H., Anguiano B., Ortíz E. A., Aragón-Salamanca A., Argudo-Fernández M., Aubert M., et al., The fifteenth data release of the Sloan Digital Sky Surveys: first release of MaNGA-derived quantities, data visualization tools, and Stellar Library, The Astrophysical Journal Supplement Series, 2019, vol. 240, p. 23

Agudo I., Thum C., Gómez J., Wiesemeyer H., A simultaneous 3.5 and 1.3 mm polarimetric survey of active galactic nuclei in the northern sky, A\&A, 2014, vol. 566, p. A59

Aharonian F., Buckley J., Kifune T., Sinnis G., High energy astrophysics with groundbased gamma ray detectors, Reports on Progress in Physics, 2008, vol. 71, p. 096901

Ajello M., Angioni R., Axelsson M., Ballet J., Barbiellini G., Bastieri D., Gonzalez J. B., Bellazzini R., Bissaldi E., Bloom E., et al., The fourth catalog of active galactic nuclei detected by the Fermi large area telescope, The Astrophysical Journal, 2020, vol. 892, p. 105

Ajello M., Romani R., Gasparrini D., Shaw M. S., Bolmer J., Cotter G., Finke J., Greiner J., Healey S., King O., et al., The cosmic evolution of Fermi BL Lacertae objects, The Astrophysical Journal, 2013, vol. 780, p. 73

Aleksic J., Ansoldi S., Antonelli L., Antoranz P., Babic A., Bangale P., Barrio J., González J. B., Bednarek W., Bernardini E., et al., Black hole lightning due to particle acceleration at subhorizon scales, Science, 2014, vol. 346, p. 1080 
Aleksić J., Ansoldi S., Antonelli L. A., Antoranz P., Babic A., Bangale P., de Almeida U. B., Barrio J., González J. B., Bednarek W., et al., Contemporaneous observations of the radio galaxy NGC 1275 from radio to very high energy $\gamma$-rays, Astronomy \& Astrophysics, 2014, vol. 564, p. A5

Aliu E., Anderhub H., Antonelli L. A., Antoranz P., Backes M., et al., Observation of pulsed $\gamma$-Rays above $25 \mathrm{GeV}$ from the Crab Pulsar with MAGIC, Science, 2008, vol. 322, p. 1221

Allen C., Variation of the Sun's ultra-violet radiation as revealed by ionospheric and geomagnetic observations, Terrestrial Magnetism and Atmospheric Electricity, 1946, vol. 51, p. 1

Almeida I., Nemmen R., Wong K.-W., Wu Q., Irwin J. A., The multiwavelength spectrum of NGC 3115: hot accretion flow properties, MNRAS, 2018, vol. 475, p. 5398

Alpar M., Cheng A., Ruderman M., Shaham J., A new class of radio pulsars, Nature, 1982, vol. 300, p. 728

Arons J., Pair creation above pulsar polar caps-Geometrical structure and energetics of slot gaps, The Astrophysical Journal, 1983, vol. 266, p. 215

Arsioli B., Fraga B., Giommi P., Padovani P., Marrese P., 1WHSP: An IR-based sample of $^{\sim} 1000$ VHE $\gamma$-ray blazar candidates, Astronomy \& Astrophysics, 2015, vol. 579, p. A34

Asmus D., Hönig S., Gandhi P., Smette A., Duschl W., The subarcsecond mid-infrared view of local active galactic nuclei-I. The N-and Q-band imaging atlas, MNRAS, 2014, vol. 439 , p. 1648

Atwood W., Abdo A. A., Ackermann M., Althouse W., Anderson B., Axelsson M., Baldini L., Ballet J., Band D., Barbiellini G., et al., The large area telescope on the Fermi gamma-ray space telescope mission, The Astrophysical Journal, 2009, vol. 697, p. 1071

Atwood W., Albert A., Baldini L., Tinivella M., Bregeon J., Pesce-Rollins M., Sgrò C., Bruel P., Charles E., Drlica-Wagner A., et al., Pass 8: toward the full realization of the Fermi-LAT scientific potential, arXiv preprint arXiv:1303.3514, 2013 
Baade W., Zwicky F., Cosmic rays from super-novae, Proceedings of the National Academy of Sciences, 1934, vol. 20, p. 259

Bahramian A., Heinke C. O., Sivakoff G. R., Gladstone J. C., Stellar encounter rate in galactic globular clusters, The Astrophysical Journal, 2013, vol. 766, p. 136

Baixeras C., Collaboration M., et al., The MAGIC telescope, Nuclear Physics BProceedings Supplements, 2003, vol. 114, p. 247

Baldwin J. A., Phillips M. M., Terlevich R., CLASSIFICATION PARAMETERS FOR THE EMISSION-LINE SPECTRA OF EXTRAGALACTIC OBJECTS., Publications of the Astronomical Society of the Pacific, 1981, vol. 93, p. 5

Barthelmy S. D., Barbier L. M., Cummings J. R., Fenimore E. E., Gehrels N., Hullinger D., Krimm H. A., Markwardt C. B., Palmer D. M., Parsons A., Sato G., Suzuki M., Takahashi T., Tashiro M., Tueller J., The Burst Alert Telescope (BAT) on the SWIFT Midex Mission, Space Sci. Rev., 2005, vol. 120, p. 143

Baumgardt H., Kroupa P., Parmentier G., The influence of residual gas expulsion on the evolution of the Galactic globular cluster system and the origin of the Population II halo, Monthly Notices of the Royal Astronomical Society, 2008, vol. 384, p. 1231

Bednarek W., Sitarek J., High-energy $\gamma$-rays from globular clusters, Monthly Notices of the Royal Astronomical Society, 2007, vol. 377, p. 920

Berlin A., Hooper D., McDermott S. D., Simplified dark matter models for the galactic center gamma-ray excess, Physical Review D, 2014, vol. 89, p. 115022

Bernieri E., Campana R., Massaro E., Paggi A., Tramacere A., A new flaring high-energy $\gamma$-ray source, Astronomy \& Astrophysics, 2013, vol. 551, p. L5

Bica E., Bonatto C., Barbuy B., Ortolani S., Globular cluster system and Milky Way properties revisited, Astronomy \& Astrophysics, 2006, vol. 450, p. 105

Blandford R., Rees M., Wolfe A., Pittsburgh conference on BL Lac objects, AM Wolfe, Ed, 1978, vol. 328

Blandford R. D., Begelman M. C., On the fate of gas accreting at a low rate on to a black hole, Monthly Notices of the Royal Astronomical Society, 1999, vol. 303, p. L1 
Bonardi A., Pühlhofer G., Hermanutz S., Santangelo A., A new solution for mirror coating in $\gamma$-ray Cherenkov astronomy, Experimental Astronomy, 2014, vol. 38, p. 1

Bondi H., On spherically symmetrical accretion, Monthly Notices of the Royal Astronomical Society, 1952, vol. 112, p. 195

Böttcher M., , 2007 in , The Multi-Messenger Approach to High-Energy Gamma-Ray Sources. Springer pp 95-104

Böttcher M., Els P., GAMMA-GAMMA ABSORPTION IN THE BROAD LINE REGION RADIATION FIELDS OF GAMMA-RAY BLAZARS, The Astrophysical Journal, 2016, vol. 821, p. 102

Breiman L., Random forests, Machine learning, 2001, vol. 45, p. 5

Brown A. M., Lacroix T., Lloyd S., Bøhm C., Chadwick P., Understanding the $\gamma$-ray emission from the globular cluster 47 Tuc: evidence for dark matter?, Physical Review D, 2018, vol. 98, p. 041301

Brown J., Burkert A., Truran J. W., On the formation of globular clusters. I-Dynamical limits on globular cluster metallicities, The Astrophysical Journal, 1991, vol. 376, p. 115

Bühler R., Blandford R., The surprising Crab pulsar and its nebula: a review, Reports on Progress in Physics, 2014, vol. 77, p. 066901

Campana R., Massaro E., Bernieri E., Application of the MST clustering to the high energy $\gamma$-ray sky. III-New detections of $\gamma$-ray emission from blazars, Astrophysics and space science, 2016, vol. 361, p. 1

Canvin J., Laing R., Bridle A., Cotton W., A relativistic model of the radio jets in NGC 315, MNRAS, 2005, vol. 363, p. 1223

Capetti A., Kleijn G. V., Chiaberge M., The HST view of the nuclear emission line region in low luminosity radio-galaxies, A\&A, 2005, vol. 439, p. 935

Caraveo P. A., Gamma-ray pulsar revolution, Annual Review of Astronomy and Astrophysics, 2014, vol. 52, p. 211 
Cardelli J. A., Clayton G. C., Mathis J. S., The relationship between infrared, optical, and ultraviolet extinction, The Astrophysical Journal, 1989, vol. 345, p. 245

Casandjian J.-M., Grenier I., A Revised Catalogue of EGRET Sources. In International Cosmic Ray Conference, vol. 2, 2008, p. 501

Chen K., Gamma-ray emission from millisecond pulsars in globular clusters, Nature, 1991, vol. 352 , p. 695

Cheng K., Chernyshov D., Dogiel V., Hui C., Kong A., The origin of gamma rays from globular clusters, The Astrophysical Journal, 2010, vol. 723, p. 1219

Cheng K., Ho C., Ruderman M., Energetic radiation from rapidly spinning pulsars. I-Outer magnetosphere gaps. II-VELA and Crab, The Astrophysical Journal, 1986, vol. 300, p. 500

Cheng K., Zhang C., Magnetic field evolution of accreting neutron stars, Astronomy and Astrophysics, 1998, vol. 337, p. 441

Chiaro G., Salvetti D., La Mura G., Giroletti M., Thompson D., Bastieri D., Blazar flaring patterns (B-FlaP) classifying blazar candidate of uncertain type in the third FermiLAT catalogue by artificial neural networks, Monthly Notices of the Royal Astronomical Society, 2016, vol. 462, p. 3180

Chubb T., Friedman H., Kreplin R., Kupperian J., Rocket observation of X-ray emission in a solar flare, Nature, 1957, vol. 179, p. 861

Clark G., Garmire G., Kraushaar W., Observation of high-energy cosmic gamma rays, The Astrophysical Journal, 1968, vol. 153, p. L203

Clemens J. C., Crain J. A., Anderson R., The Goodman spectrograph. In Ground-based Instrumentation for Astronomy, vol. 5492, 2004, p. 331

Condon J. J., Cotton W., Greisen E., Yin Q., Perley R., Taylor G., Broderick J., The NRAO VLA sky survey, The Astronomical Journal, 1998, vol. 115, p. 1693

Costamante L., Bonnoli G., Tavecchio F., Ghisellini G., Tagliaferri G., Khangulyan D., The NuSTAR view on hard-TeV BL Lacs, MNRAS, 2018, vol. 477, p. 4257 
Cotton W., Feretti L., Giovannini G., Lara L., Venturi T., A Parsec-Scale Accelerating Radio Jet in the Giant Radio Galaxy NGC 315, ApJ, 1999, vol. 519, p. 108

Cox D. R., Regression Models and Life-Tables, Journal of the Royal Statistical Society. Series B (Methodological), 1972, vol. 34, p. 187

Craig M., Crawford S., Deil C., Gomez C., Günther H. M., Heidt N., Horton A., Karr J., Nelson S., Ninan J. P., et al., ccdproc: CCD data reduction software, Astrophysics Source Code Library, 2015

Crespo N. Á., Masetti N., Ricci F., Landoni M., Patiño-Álvarez V., Massaro F., D’Abrusco R., Paggi A., Chavushyan V., Jiménez-Bailón E., et al., Optical spectroscopic observations of gamma-ray blazar candidates. V. TNG, KPNO, and OAN observations of blazar candidates of uncertain type in the Northern Hemisphere, The Astronomical Journal, 2016, vol. 151, p. 32

Cusumano G., La Parola V., Segreto A., Ferrigno C., Maselli A., Sbarufatti B., Romano P., Chincarini G., Giommi P., Masetti N., Moretti A., Parisi P., Tagliaferri G., The Palermo Swift-BAT hard X-ray catalogue. III. Results after 54 months of sky survey, A\&A, 2010, vol. 524, p. A64

Cutri R., Wright E., Conrow T., Fowler J., Eisenhardt P., Grillmair C., Kirkpatrick J., Masci F., McCallon H., Wheelock S., et al., Explanatory Supplement to the AllWISE Data Release Products, Explanatory Supplement to the AllWISE Data Release Products, by RM Cutri et al., 2013

Cutri R. e., et al., VizieR online data catalog: AllWISE data release (cutri+ 2013), VizieR Online Data Catalog, 2014, vol. 2328

D’Abrusco R., Crespo N. Á., Massaro F., Campana R., Chavushyan V., Landoni M., Franca F. L., Masetti N., Milisavljevic D., Paggi A., Ricci F., Smith H. A., Two New Catalogs of Blazar Candidates in the WISE Infrared Sky, The Astrophysical Journal Supplement Series, 2019, vol. 242, p. 4

D’Abrusco R., Massaro F., Ajello M., Grindlay J., Smith H. A., Tosti G., Infrared colors of the gamma-ray-detected blazars, The Astrophysical Journal, 2012, vol. 748, p. 68 
D’Abrusco R., Massaro F., Paggi A., Masetti N., Tosti G., Giroletti M., Smith H., Unveiling the nature of unidentified gamma-ray sources. I. A new method for the association of gamma-ray blazars, The Astrophysical Journal Supplement Series, 2013, vol. 206, p. 12

D'Abrusco R., Massaro F., Paggi A., Smith H., Masetti N., Landoni M., Tosti G., The WISE Blazar-like Radio-loud Sources: An All-sky Catalog of Candidate $\gamma$-ray Blazars, The Astrophysical Journal Supplement Series, 2014, vol. 215, p. 14

de Menezes R., Amaya-Almazán R. A., Marchesini E. J., Peña-Herazo H. A., Massaro F., Chavushyan V., Paggi A., Landoni M., Masetti N., Ricci F., et al., Optical spectroscopic observations of gamma-ray blazar candidates. X. Results from the 2018-2019 SOAR and OAN-SPM observations of blazar candidates of uncertain type, Astrophysics and Space Science, 2020, vol. 365, p. 1

de Menezes R., Cafardo F., Nemmen R., Milky Way globular clusters in $\gamma$-rays: analysing the dynamical formation of millisecond pulsars, Monthly Notices of the Royal Astronomical Society, 2019, vol. 486, p. 851

de Menezes R., D’Abrusco R., Massaro F., Gasparrini D., Nemmen R., On the physical association of Fermi-LAT blazars with their low-energy counterparts, The Astrophysical Journal Supplement Series, 2020, vol. 248, p. 23

de Menezes R., Nemmen R., Finke J. D., Almeida I., Rani B., Gamma-ray observations of low-luminosity active galactic nuclei, MNRAS, 2020, vol. 492, p. 4120

de Menezes R., Peña-Herazo H. A., Marchesini E. J., D’Abrusco R., Masetti N., Nemmen R., Massaro F., Ricci F., Landoni M., Paggi A., et al., Optical characterization of WISE selected blazar candidates, Astronomy \& Astrophysics, 2019, vol. 630, p. A55

DeYoung T., Collaboration H., et al., The HAWC observatory, Nuclear Instruments and Methods in Physics Research Section A: Accelerators, Spectrometers, Detectors and Associated Equipment, 2012, vol. 692, p. 72

Doert M., Errando M., Search for gamma-ray-emitting active galactic nuclei in the FermiLAT unassociated sample using machine learning, The Astrophysical Journal, 2014, vol. 782 , p. 41 
Dressler A., Shectman S. A., Systematics of the 4000 Angstrom Break in the Spectra of Galaxies, The Astronomical Journal, 1987, vol. 94, p. 899

Edmonds P. D., Gilliland R. L., Heinke C. O., Grindlay J. E., An extensive census of Hubble Space Telescope counterparts to Chandra X-ray sources in the globular cluster 47 Tucanae. II. Time series and analysis, The Astrophysical Journal, 2003, vol. 596, p. 1197

Event Horizon Telescope Collaboration Akiyama K., Alberdi A., Alef W., Asada K., Azulay R., Baczko A.-K., et al., First M87 Event Horizon Telescope Results. I. The Shadow of the Supermassive Black Hole, ApJ, 2019, vol. 875, p. L1

Fabian A., Walker S., Pinto C., Russell H., Edge A., Effects of the variability of the nucleus of NGC 1275 on X-ray observations of the surrounding intracluster medium, MNRAS, 2015, vol. 451, p. 3061

Fanaroff B., Riley J., The morphology of extragalactic radio sources of high and low luminosity, MNRAS, 1974, vol. 167, p. 31P

Feigelson E. D., Babu G. J., Modern Statistical Methods for Astronomy. Cambridge University Press, 2012

Fermi LAT Collaboration Fermi Gamma-Ray Imaging of a Radio Galaxy, Science, 2010a, vol. 328 , p. 725

Fermi LAT Collaboration Fermi Gamma-Ray Imaging of a Radio Galaxy, Science, 2010b, vol. 328 , p. 725

Ferrarese L., Ford H. C., Jaffe W., Evidence for a massive black hole in the active galaxy NGC 4261 from Hubble Space Telescope images and spectra, ApJ, 1996, vol. 470, p. 444

Fichtel C., Bertsch D., Chiang J., Dingus B., Esposito J., Fierro J., Hartman R., Hunter S., Kanbach G., Kniffen D., et al., The first energetic gamma-ray experiment telescope (EGRET) source catalog, The Astrophysical Journal Supplement Series, 1994, vol. 94, p. 551

Finke J. D., Compton dominance and the blazar sequence, The Astrophysical Journal, 2013, vol. 763, p. 134 
Finke J. D., Dermer C. D., Böttcher M., Synchrotron self-Compton analysis of TeV X-rayselected BL Lacertae objects, The Astrophysical Journal, 2008, vol. 686, p. 181

Fireman E., Interstellar absorption of x-rays, The Astrophysical Journal, 1974, vol. 187, p. 57

Fregeau J. M., Gürkan M., Joshi K., Rasio F., Monte Carlo simulations of globular cluster evolution. III. Primordial binary interactions, The Astrophysical Journal, 2003, vol. 593, p. 772

Freire P., Abdo A., Ajello M., Allafort A., Ballet J., Barbiellini G., Bastieri D., Bechtol K., Bellazzini R., Blandford R., et al., Fermi detection of a luminous $\gamma$-ray pulsar in a globular cluster, Science, 2011, vol. 334, p. 1107

Freire P. C., The pulsar population in Globular Clusters and in the Galaxy, Proceedings of the International Astronomical Union, 2012, vol. 8, p. 243

Genzel R., Eisenhauer F., Gillessen S., The Galactic Center massive black hole and nuclear star cluster, Reviews of Modern Physics, 2010, vol. 82, p. 3121

Ghisellini G., Tavecchio F., Fermi/LAT broad emission line blazars, MNRAS, 2015, vol. 448 , p. 1060

Gonzalez-Martin O., Masegosa J., Márquez I., Guerrero M. A., Dultzin-Hacyan D., X-ray nature of the LINER nuclear sources, A\&A, 2006, vol. 460, p. 45

Grandi P., Torresi E., De Rosa A., Rainó S., Malaguti G., Fermi-LAT Gamma-Ray Variability Study of Misaligned AGN. In EPJ Web of Conferences , vol. 61, 2013, p. 04007

Gu Q.-S., Huang J.-S., Wilson G., Fazio G., Direct Evidence from Spitzer for a lowluminosity AGN at the center of the Elliptical Galaxy NGC 315, ApJ, 2007, vol. 671, p. L105

Halpern J., Steiner J., Low-ionization active galactic nuclei-X-ray or shock heated?, The Astrophysical Journal, 1983, vol. 269, p. L37

Hansen B. M., Phinney E. S., The pulsar kick velocity distribution, Monthly Notices of the Royal Astronomical Society, 1997, vol. 291, p. 569 
Harding A. K., Tademaru E., Esposito L., A curvature-radiation-pair-production model for gamma-ray pulsars, The Astrophysical Journal, 1978, vol. 225, p. 226

Harding A. K., Usov V. V., Muslimov A. G., High-energy emission from millisecond pulsars, The Astrophysical Journal, 2005, vol. 622, p. 531

Harris W. E., A catalog of parameters for globular clusters in the Milky Way, The Astronomical Journal, 1996, vol. 112, p. 1487

Hartman R., Bertsch D., Bloom S., Chen A., Deines-Jones P., Esposito J., Fichtel C., Friedlander D., Hunter S., McDonald L., et al., The third EGRET catalog of high-energy gamma-ray sources, The Astrophysical Journal Supplement Series, 1999, vol. 123, p. 79

Haşegan M., Jordán A., Côté P., Djorgovski S., McLaughlin D. E., Blakeslee J. P., Mei S., West M. J., Peng E. W., Ferrarese L., et al., The ACS virgo cluster survey. VII. Resolving the connection between globular clusters and ultracompact dwarf galaxies, The Astrophysical Journal, 2005, vol. 627, p. 203

Hassan T., Arrabito L., Bernlöhr K., Bregeon J., Cortina J., Cumani P., Di Pierro F., Falceta-Goncalves D., Lang R. G., Hinton J., et al., Monte Carlo performance studies for the site selection of the Cherenkov Telescope Array, Astroparticle Physics, 2017, vol. 93 , p. 76

Hassan T., Arrabito L., Bernlör K., Bregeon J., Hinton J., Jogler T., Maier G., Moralejo A., Di Pierro F., Wood M., et al., Second large-scale Monte Carlo study for the Cherenkov telescope array, arXiv preprint arXiv:1508.06075, 2015

Hassan T., Mirabal N., Contreras J., Oya I., Gamma-ray active galactic nucleus type through machine-learning algorithms, Monthly Notices of the Royal Astronomical Society, 2012, vol. 428 , p. 220

Heinke C. O., Grindlay J. E., Edmonds P. D., Cohn H. N., Lugger P. M., Camilo F., Bogdanov S., Freire P. C., A Deep Chandra Survey of the Globular Cluster 47 Tucanae: Catalog of Point Sources, The Astrophysical Journal, 2005, vol. 625, p. 796

Helfand D. J., White R. L., Becker R. H., The last of FIRST: The final catalog and source identifications, The Astrophysical Journal, 2015, vol. 801, p. 26 
Herterich K., Absorption of gamma rays in intense X-ray sources, Nature, 1974, vol. 250, p. 311

Hewish A., Bell S. J., Pilkington J. D., Scott P. F., Collins R. A., Observation of a rapidly pulsating radio source, Nature, 1968, vol. 217, p. 709

Hinton J. A., Collaboration H., et al., The status of the HESS project, New Astronomy Reviews, 2004, vol. 48, p. 331

Hirotani K., High Energy Emission from Rotation-Powered Pulsars: Outer-gap vs. Slot-gap Models, arXiv preprint arXiv:0809.1283, 2008a

Hirotani K., Outer-gap versus slot-gap models for pulsar high-energy emissions: the case of the Crab pulsar, The Astrophysical Journal Letters, 2008b, vol. 688, p. L25

Hirotani K., Very High-Energy Emission from the Direct Vicinity of Rapidly Rotating Black Holes, Galaxies, 2018, vol. 6, p. 122

Ho L. C., Nuclear activity in nearby galaxies, Annu. Rev. Astron. Astrophys., 2008, vol. 46, p. 475

Ho L. C., Radiatively inefficient accretion in nearby galaxies, The Astrophysical Journal, 2009, vol. 699, p. 626

Ho L. C., Filippenko A. V., Sargent W. L., A search for 'dwarf' Seyfert nuclei. 2: an optical spectral atlas of the nuclei of nearby galaxies, ApJS, 1995, vol. 98, p. 477

Ho L. C., Filippenko A. V., Sargent W. L., A Search for "Dwarf"Seyfert Nuclei. V. Demographics of Nuclear Activity in Nearby Galaxies, The Astrophysical Journal, 1997, vol. 487 , p. 568

Holder J., Atkins R., Badran H., Blaylock G., Bradbury S., Buckley J., Byrum K., CarterLewis D., Celik O., Chow Y., et al., The first VERITAS telescope, Astroparticle Physics, 2006, vol. 25, p. 391

Hooper D., Linden T., The gamma-ray pulsar population of globular clusters: implications for the GeV excess, Journal of Cosmology and Astroparticle Physics, 2016, vol. 2016, p. 018 
Hui C., Cheng K., Taam R. E., Dynamical formation of millisecond pulsars in globular clusters, The Astrophysical Journal, 2010, vol. 714, p. 1149

Hui C., Cheng K., Wang Y., Tam P., Kong A., Chernyshov D., Dogiel V., The Fundamental Plane of Gamma-ray Globular Clusters, The Astrophysical Journal, 2010, vol. 726, p. 100

Ichimaru S., Bimodal behavior of accretion disks: theory and application to Cygnus X-1 transitions., ApJ, 1977, vol. 214, p. 840

Isobe T., Feigelson E. D., Nelson P. I., Statistical Methods for Astronomical Data with Upper Limits. II. Correlation and Regression, The Astrophysical Journal, 1986, vol. 306, p. 490

Jackson J. D., , 1999 Classical electrodynamics

James G., Witten D., Hastie T., Tibshirani R., An introduction to statistical learning. vol. 112, Springer, 2013

Jansky K. G., Directional studies of atmospherics at high frequencies, Proceedings of the Institute of Radio Engineers, 1932, vol. 20, p. 1920

Jauch J. M., Rohrlich F., The theory of photons and electrons: the relativistic quantum field theory of charged particles with spin one-half. Springer Science \& Business Media, 2012

Johnson T. J., Guillemot L., Kerr M., Cognard I., Ray P. S., Wolff M., Begin S., Janssen G., Romani R., Venter C., et al., Broadband pulsations from PSR B1821-24: Implications for emission models and the pulsar population of M28, The Astrophysical Journal, 2013, vol. 778 , p. 106

Jones D. L., Wehrle A. E., VLBA Imaging of NGC 4261: Symmetric Parsec-scale Jets and the Inner Accretion Region, ApJ, 1997, vol. 484, p. 186

Junor W., Biretta J. A., Livio M., Formation of the radio jet in M87 at 100 Schwarzschild radii from the central black hole, Nature, 1999, vol. 401, p. 891 
Kafexhiu E., Aharonian F., Taylor A. M., Vila G. S., Parametrization of gamma-ray production cross sections for $\mathrm{p} p$ interactions in a broad proton energy range from the kinematic threshold to PeV energies, Phys. Rev. D, 2014, vol. 90, p. 123014

Kelly B. C., Some aspects of measurement error in linear regression of astronomical data, The Astrophysical Journal, 2007, vol. 665, p. 1489

Kewley L. J., Groves B., Kauffmann G., Heckman T., The host galaxies and classification of active galactic nuclei, Monthly Notices of the Royal Astronomical Society, 2006, vol. 372, p. 961

Khiali B., de Gouveia Dal Pino E. M., Sol H., Particle Acceleration and gamma-ray emission due to magnetic reconnection around the core region of radio galaxies, ArXiv eprints, 2015

King I., The structure of star clusters. I. an empirical density law, The Astronomical Journal, 1962, vol. 67, p. 471

King I. R., The structure of star clusters. III. Some simple dynamical models, The Astronomical Journal, 1966, vol. 71, p. 64

Koratkar A., Blaes O., The ultraviolet and optical continuum emission in active galactic nuclei: the status of accretion disks, Publications of the Astronomical Society of the Pacific, 1999, vol. 111, p. 1

Kovačević M., Chiaro G., Cutini S., Tosti G., Optimizing neural network techniques in classifying Fermi-LAT gamma-ray sources, Monthly Notices of the Royal Astronomical Society, 2019, vol. 490, p. 4770

Kraushaar W., Clark G., Search for primary cosmic gamma rays with the satellite Explorer XI, Physical Review Letters, 1962, vol. 8, p. 106

Kraushaar W., Clark G., Garmire G., Borken R., Higbie P., Leong V., Thorsos T., Highenergy cosmic gamma-ray observations from the OSO-3 satellite, The Astrophysical Journal, 1972, vol. 177, p. 341

Kraushaar W., Clark G. W., Garmire G., Helmken H., Higbie P., Agogino M., Explorer XI Experiment on Cosmic Gamma Rays., ApJ, 1965, vol. 141, p. 845 
Landis G. A., Mission to the gravitational focus of the sun: A critical analysis. In 55th AIAA Aerospace Sciences Meeting , 2017, p. 1679

Landoni M., Falomo R., Treves A., Sbarufatti B., Spectroscopy of BL Lacertae objects of extraordinary luminosity, Astronomy \& Astrophysics, 2014, vol. 570, p. A126

Landoni M., Falomo R., Treves A., Scarpa R., Payá D. R., What is the redshift of the gamma-ray BL Lac source S4 0954+ 65?, The Astronomical Journal, 2015, vol. 150, p. 181

Landoni M., Massaro F., Paggi A., D’Abrusco R., Milisavljevic D., Masetti N., Smith H., Tosti G., Chomiuk L., Strader J., et al., Optical Spectroscopic Observations of $\gamma$ Ray Blazar Candidates. III. The 2013/2014 Campaign in the Southern Hemisphere, The Astronomical Journal, 2015a, vol. 149, p. 163

Landoni M., Massaro F., Paggi A., D’Abrusco R., Milisavljevic D., Masetti N., Smith H., Tosti G., Chomiuk L., Strader J., et al., Optical Spectroscopic Observations of $\gamma$ Ray Blazar Candidates. III. The 2013/2014 Campaign in the Southern Hemisphere, The Astronomical Journal, 2015b, vol. 149, p. 163

Landoni M., Paiano S., Falomo R., Scarpa R., Treves A., High-redshift BL Lac Objects: Spectroscopy of Candidates, The Astrophysical Journal, 2018, vol. 861, p. 130

Landt H., Padovani P., Giommi P., The classification of BL Lacertae objects: the Ca H\&K break, Monthly Notices of the Royal Astronomical Society, 2002, vol. 336, p. 945

Lavalley M., Isobe T., Feigelson E., ASURV: Astronomy Survival Analysis Package. In Astronomical Data Analysis Software and Systems I, vol. 25 of Astronomical Society of the Pacific Conference Series, 1992, p. 245

Lazio T. J. W., Waltman E. B., Ghigo F. D., Fiedler R. L., Foster R. S., Johnston K. J., A Dual-Frequency, Multiyear Monitoring Program of Compact Radio Sources, ApJS, 2001, vol. 136 , p. 265

Lee J.-W., Kang Y.-W., Lee J., Lee Y.-W., Enrichment by supernovae in globular clusters with multiple populations, Nature, 2009, vol. 462, p. 480 
Lefaucheur J., Pita S., Research and characterisation of blazar candidates among the Fermi/LAT 3FGL catalogue using multivariate classifications, Astronomy \& Astrophysics, 2017, vol. 602, p. A86

Levinson A., Rieger F., Variable TeV emission as a manifestation of jet formation in M87?, The Astrophysical Journal, 2011, vol. 730, p. 123

Liu H., Wu Q., Possible Origin of Radio Emission from Nonthermal Electrons in Hot Accretion Flows for Low-luminosity Active Galactic Nuclei, ApJ, 2013, vol. 764, p. 17

Lloyd S. J., Chadwick P. M., Brown A. M., Gamma-ray emission from high Galactic latitude globular clusters, Monthly Notices of the Royal Astronomical Society, 2018, vol. 480 , p. 4782

Lorimer D., Kramer M., Ellis R., Huchra J., Kahn S., Rieke G., Stetson P., , 2005 Cambridge Observing Handbooks for Research Astronomers

Lorimer D. R., Binary and Millisecond Pulsars at the New Millenium, Living Reviews in Relativity, 2001, vol. 4, p. 5

Lorimer D. R., Kramer M., Handbook of pulsar astronomy, Handbook of pulsar astronomy, by DR Lorimer and M. Kramer. Cambridge observing handbooks for research astronomers, Vol. 4. Cambridge, UK: Cambridge University Press, 2004, 2004, vol. 4

Ly C., Walker R. C., Wrobel J., An attempt to probe the radio jet collimation regions in NGC 4278, NGC 4374 (M84), and NGC 6166, AJ, 2004, vol. 127, p. 119

Lynden-Bell D., Pringle J. E., The evolution of viscous discs and the origin of the nebular variables, Monthly Notices of the Royal Astronomical Society, 1974, vol. 168, p. 603

McClintock J. E., Narayan R., Steiner J. F., , 2013 in , The Physics of Accretion onto Black Holes. Springer pp 295-322

McEnery J., Barrio J. A., Agudo I., Ajello M., Álvarez J.-M., Ansoldi S., Anton S., Auricchio N., Stephen J. B., Baldini L., et al., All-sky Medium Energy Gamma-ray Observatory: Exploring the Extreme Multimessenger Universe, arXiv preprint arXiv:1907.07558, 2019 
McGlynn T., Scollick K., White N., Skyview: The Multi-Wavelength Sky on the Interne. In Symposium-International Astronomical Union, vol. 179, 1998, p. 465

Madrid J. P., Donzelli C. J., Rodríguez-Ardila A., Paggi A., Massaro F., Schirmer M., 3C 17: The BCG of a Galaxy Cluster at $\mathrm{z}=0.22$, The Astrophysical Journal Supplement Series, 2018, vol. 238, p. 31

Mahadevan R., Scaling laws for advection-dominated flows: applications to low-luminosity galactic nuclei, The Astrophysical Journal, 1997, vol. 477, p. 585

Mahadevan R., Narayan R., Krolik J., Gamma-ray emission from advection-dominated accretion flows around black holes: application to the galactic center, The Astrophysical Journal, 1997, vol. 486, p. 268

Mainzer A., Grav T., Bauer J., Masiero J., McMillan R., Cutri R., Walker R., Wright E., Eisenhardt P., Tholen D., et al., NEOWISE observations of near-Earth objects: preliminary results, The Astrophysical Journal, 2011, vol. 743, p. 156

Marchesini E., Peña-Herazo H., Crespo N. Á., Ricci F., Negro M., Milisavljevic D., Massaro F., Masetti N., Landoni M., Chavushyan V., et al., Optical spectroscopic observations of gamma-ray blazar candidates VIII: the 2016-2017 follow up campaign carried out at SPM, NOT, KPNO and SOAR telescopes, Astrophysics and Space Science, 2019, vol. 364 , p. 5

Markoff S., Falcke H., Yuan F., Biermann P. L., The Nature of the 10 kilosecond X-ray flare in Sgr A, Astronomy \& Astrophysics, 2001, vol. 379, p. L13

Markoff S., Nowak M., Corbel S., Fender R., Falcke H., Exploring the role of jets in the radio/X-ray correlations of GX 339-4, A\&A, 2003, vol. 397, p. 645

Markoff S., Nowak M. A., Wilms J., Going with the Flow: Can the Base of Jets Subsume the Role of Compact Accretion Disk Coronae?, ApJ, 2005, vol. 635, p. 1203

Masci F. J., Condon J., Barlow T., Lonsdale C., Xu C., Shupe D., Pevunova O., Fang F., Cutri R., A New Complete Sample of Submillijansky Radio Sources: An Optical and Near-Infrared Study, Publications of the Astronomical Society of the Pacific, 2001, vol. 113 , p. 10 
Massaro E., Maselli A., Leto C., Marchegiani P., Perri M., Giommi P., Piranomonte S., The 5th edition of the Roma-BZCAT. A short presentation, Astrophysics and Space Science, 2015, vol. 357, p. 75

Massaro E., Nesci R., Piranomonte S., The colours of BL Lac objects: a new approach to their classification, Monthly Notices of the Royal Astronomical Society, 2012, vol. 422, p. 2322

Massaro F., Crespo N. Á., D’Abrusco R., Landoni M., Masetti N., Ricci F., Milisavljevic D., Paggi A., Chavushyan V., Jiménez-Bailón E., et al., The gamma-ray blazar quest: new optical spectra, state of art and future perspectives, Astrophysics and Space Science, 2016a, vol. 361, p. 337

Massaro F., Crespo N. Á., D’Abrusco R., Landoni M., Masetti N., Ricci F., Milisavljevic D., Paggi A., Chavushyan V., Jiménez-Bailón E., et al., The gamma-ray blazar quest: new optical spectra, state of art and future perspectives, Astrophysics and Space Science, 2016b, vol. 361, p. 337

Massaro F., D'Abrusco R., Ajello M., Grindlay J., Smith H. A., Identification of the infrared non-thermal emission in blazars, The Astrophysical Journal Letters, 2011a, vol. 740 , p. L48

Massaro F., D’Abrusco R., Ajello M., Grindlay J., Smith H. A., Identification of the infrared non-thermal emission in blazars, The Astrophysical Journal Letters, 2011b, vol. 740 , p. L48

Massaro F., D’Abrusco R., Landoni M., Paggi A., Masetti N., Giroletti M., Otí-Floranes H., Chavushyan V., Jiménez-Bailón E., Patiño-Álvarez V., et al., Refining the associations of the Fermi large area telescope source catalogs, The Astrophysical Journal Supplement Series, 2015, vol. 217, p. 2

Massaro F., D’Abrusco R., Paggi A., Masetti N., Giroletti M., Tosti G., Smith H. A., Funk S., Unveiling the nature of the unidentified gamma-ray sources. V. Analysis of the radio candidates with the kernel density estimation, The Astrophysical Journal Supplement Series, 2013, vol. 209, p. 10 
Massaro F., D’Abrusco R., Tosti G., Ajello M., Gasparrini D., Grindlay J., Smith H. A., The WISE gamma-ray strip parameterization: the nature of the gamma-ray active galactic nuclei of uncertain type, The Astrophysical Journal, 2012, vol. 750, p. 138

Massaro F., Harris D., Chiaberge M., Grandi P., Macchetto F., Baum S., O’Dea C., Capetti A., The Jet of 3C 17 and the Use of Jet Curvature as a Diagnostic of the X-ray Emission Process, The Astrophysical Journal, 2009, vol. 696, p. 980

Massaro F., Harris D., Tremblay G., Axon D., Baum S., Capetti A., Chiaberge M., Gilli R., Giovannini G., Grandi P., et al., Chandra Observations of 3C Radio Sources with zi 0.3: Nuclei, Diffuse Emission, Jets, and Hotspots, The Astrophysical Journal, 2010, vol. 714 , p. 589

Massaro F., Landoni M., D’Abrusco R., Milisavljevic D., Paggi A., Masetti N., Smith H. A., Tosti G., Optical spectroscopic observations of $\gamma$-ray blazar candidates-II. The 2013 KPNO campaign in the northern hemisphere, Astronomy \& Astrophysics, 2015, vol. 575, p. A124

Massaro F., Marchesini E. J., D’Abrusco R., Masetti N., Andruchow I., Smith H. A., Radioweak BL Lac Objects in the Fermi Era, The Astrophysical Journal, 2017, vol. 834, p. 113

Massaro F., Masetti N., D'Abrusco R., Paggi A., Funk S., Optical spectroscopic observations of blazars and $\gamma$-Ray blazar candidates in the sloan digital sky survey data release nine, The Astronomical Journal, 2014, vol. 148, p. 66

Massaro F., Thompson D. J., Ferrara E. C., The extragalactic gamma-ray sky in the Fermi era, The Astronomy and Astrophysics Review, 2016, vol. 24, p. 2

Mattox J., Schachter J., Molnar L., Hartman R., Patnaik A., The identification of EGRET sources with flat-spectrum radio sources, The Astrophysical Journal, 1997, vol. 481, p. 95

Mattox J. R., Bertsch D., Chiang J., Dingus B., Digel S., Esposito J., Fierro J., Hartman R., Hunter S., Kanbach G., et al., The likelihood analysis of EGRET data, The Astrophysical Journal, 1996, vol. 461, p. 396 
Mauch T., Murphy T., Buttery H., Curran J., Hunstead R., Piestrzynski B., Robertson J., Sadler E., SUMSS: a wide-field radio imaging survey of the southern sky-II. The source catalogue, Monthly Notices of the Royal Astronomical Society, 2003, vol. 342, p. 1117

Max-Moerbeck W., Hovatta T., Richards J., King O., Pearson T., Readhead A., Reeves R., Shepherd M., Stevenson M., Angelakis E., et al., Time correlation between the radio and gamma-ray activity in blazars and the production site of the gamma-ray emission, Monthly Notices of the Royal Astronomical Society, 2014, vol. 445, p. 428

Meegan C., Lichti G., Bhat P., Bissaldi E., Briggs M. S., Connaughton V., Diehl R., Fishman G., Greiner J., Hoover A. S., et al., The Fermi gamma-ray burst monitor, The Astrophysical Journal, 2009, vol. 702, p. 791

Mezcua M., Prieto M., Evidence of parsec-scale jets in low-luminosity active galactic nuclei, The Astrophysical Journal, 2014, vol. 787, p. 62

Michelson P., Bertsch D., Brazier K., Chiang J., Dingus B., Fichtel C., Fierro J., Hartman R., Hunter S., Kanbach G., et al., EGRET upper limits to the high-energy gamma-ray emission from the millisecond pulsars in nearby globular clusters, The Astrophysical Journal, 1994, vol. 435, p. 218

Mirabal N., Frias-Martinez V., Hassan T., Frias-Martinez E., Fermi's Sibyl: Mining the gamma-ray sky for dark matter subhaloes, Monthly Notices of the Royal Astronomical Society: Letters, 2012, vol. 424, p. L64

Monet D. G., Levine S. E., Canzian B., Ables H. D., Bird A. R., Dahn C. C., Guetter H. H., Harris H. C., Henden A. A., Leggett S. K., et al., The usno-b catalog, The Astronomical Journal, 2003, vol. 125, p. 984

Moore B., Constraints on the global mass-to-light ratios and on the extent of dark matter halos in globular clusters and dwarf spheroidals, The Astrophysical Journal Letters, 1996, vol. 461, p. L13

Morganti R., Killeen N., Tadhunter C., The radio structures of southern 2-Jy radio sources, Monthly Notices of the Royal Astronomical Society, 1993, vol. 263, p. 1023 
Mundell C., Wrobel J., Pedlar A., Gallimore J., The nuclear regions of the seyfert galaxy NGC 4151: parsec-scale HI absorption and a remarkable radio jet, ApJ, 2003, vol. 583, p. 192

Nagar N. M., Falcke H., Wilson A. S., Radio sources in low-luminosity active galactic nuclei-IV. Radio luminosity function, importance of jet power, and radio properties of the complete Palomar sample, Astronomy \& Astrophysics, 2005, vol. 435, p. 521

Nagar N. M., Falcke H., Wilson A. S., Ho L. C., Radio sources in low-luminosity active galactic nuclei. I. VLA detections of compact, flat-spectrum cores, The Astrophysical Journal, 2000, vol. 542, p. 186

Narayan R., Yi I., Advection-dominated accretion: A self-similar solution, arXiv preprint astro-ph/9403052, 1994a

Narayan R., Yi I., Advection-dominated accretion: Underfed black holes and neutron stars, arXiv preprint astro-ph/9411059, 1994b

Neighbors A., Doolittle R., Halpers R., The high energy astronomy observatories. In 13th Annual Meeting and Technical Display Incorporating the Forum on the Future of Air Transportation , 1977, p. 282

Nemmen R., de Menezes R., Paschalidis V., Search for QPOs in Perseus with Fermi LAT, arXiv e-prints, 2018, p. arXiv:1811.07215

Nemmen R. S., Bower R. G., Babul A., Storchi-Bergmann T., Models for jet power in elliptical galaxies: a case for rapidly spinning black holes, Monthly Notices of the Royal Astronomical Society, 2007, vol. 377, p. 1652

Nemmen R. S., Storchi-Bergmann T., Eracleous M., Spectral models for low-luminosity active galactic nuclei in LINERs: the role of advection-dominated accretion and jets, MNRAS, 2014, vol. 438, p. 2804

Nemmen R. S., Storchi-Bergmann T., Yuan F., Eracleous M., Terashima Y., Wilson A. S., Radiatively Inefficient Accretion Flow in the Nucleus of NGC 1097, ApJ, 2006, vol. 643, p. 652 
Nichols E. F., On the heat radiation of Arcturus, Vega, Jupiter, and Saturn., The Astrophysical Journal, 1901, vol. 13

Nolan P. L., Abdo A., Ackermann M., Ajello M., Allafort A., Antolini E., Atwood W., Axelsson M., Baldini L., Ballet J., et al., Fermi large area telescope second source catalog, The Astrophysical Journal Supplement Series, 2012, vol. 199, p. 31

Novikov I. D., Thorne K. S., Astrophysics of black holes.. In Black Holes (Les Astres Occlus) , 1973, p. 343

Ohsuga K., Mori M., Nakamoto T., Mineshige S., Supercritical accretion flows around black holes: two-dimensional, radiation pressure-dominated disks with photon trapping, The Astrophysical Journal, 2005, vol. 628, p. 368

Oka K., Manmoto T., Gamma-ray emission from an accretion flow around a Kerr black hole, Monthly Notices of the Royal Astronomical Society, 2003, vol. 340, p. 543

Ostriker J., Gunn J., On the nature of pulsars. I. Theory, The Astrophysical Journal, 1969, vol. 157 , p. 1395

Paggi A., Massaro F., D’Abrusco R., Smith H., Masetti N., Giroletti M., Tosti G., Funk S., Unveiling the nature of the unidentified gamma-ray sources. IV. The Swift catalog of potential X-ray counterparts, The Astrophysical Journal Supplement Series, 2013, vol. 209 , p. 9

Paggi A., Milisavljevic D., Masetti N., Jiménez-Bailón E., Chavushyan V., D’Abrusco R., Massaro F., Giroletti M., Smith H. A., Margutti R., et al., Optical spectroscopic observations of $\gamma$-ray blazar candidates. I. Preliminary results, The Astronomical Journal, 2014, vol. 147, p. 112

Paiano S., Falomo R., Treves A., Franceschini A., Scarpa R., Optical spectroscopic survey of a sample of Unidentified Fermi objects: II, The Astrophysical Journal, 2019, vol. 871, p. 162

Parkinson P. S., Xu H., Yu P., Salvetti D., Marelli M., Falcone A. D., Classification and ranking of Fermi LAT gamma-ray sources from the 3FGL catalog using machine learning techniques, The Astrophysical Journal, 2016, vol. 820, p. 8 
Pedregosa F., Varoquaux G., Gramfort A., Michel V., Thirion B., Grisel O., Blondel M., Prettenhofer P., Weiss R., Dubourg V., Vanderplas J., Passos A., Cournapeau D., Brucher M., Perrot M., Duchesnay E., Scikit-learn: Machine Learning in Python, Journal of Machine Learning Research, 2011, vol. 12, p. 2825

Peña-Herazo H., Massaro F., Chavushyan V., Marchesini E., Paggi A., Landoni M., Masetti N., Ricci F., D’Abrusco R., Milisavljevic D., et al., Optical spectroscopic observations of gamma-ray blazar candidates. IX. Optical archival spectra and further observations from SOAR and OAGH, Astrophysics and Space Science, 2019, vol. 364, p. 85

Peña-Herazo H. A., Marchesini E. J., Crespo N. Á., Ricci F., Massaro F., Chavushyan V., Landoni M., Strader J., Chomiuk L., Cheung C., et al., Optical spectroscopic observations of gamma-ray blazar candidates. VII. Follow-up campaign in the southern hemisphere, Astrophysics and Space Science, 2017, vol. 362, p. 228

Peterson L., Winckler J., Gamma-ray burst from a solar flare, Journal of Geophysical Research, 1959, vol. 64, p. 697

Petropoulou M., Giannios D., Sironi L., Blazar flares powered by plasmoids in relativistic reconnection, MNRAS, 2016, vol. 462, p. 3325

Piner B. G., Jones D. L., Wehrle A. E., Orientation and speed of the parsec-scale jet in NGC 4261 (3C 270), AJ, 2001, vol. 122, p. 2954

Pooley D., Lewin W. H., Anderson S. F., Baumgardt H., Filippenko A. V., Gaensler B. M., Homer L., Hut P., Kaspi V. M., Makino J., et al., Dynamical formation of close binary systems in globular clusters, The Astrophysical Journal Letters, 2003, vol. 591, p. L131

Price-Whelan A. M., Sipőcz B., Günther H., Lim P., Crawford S., Conseil S., Shupe D., Craig M., Dencheva N., Ginsburg A., et al., The astropy project: building an openscience project and status of the v2. 0 core package, The Astronomical Journal, 2018, vol. 156 , p. 123

Quataert E., Radiatively inefficient accretion flow models of Sgr A, Astronomische Nachrichten: Astronomical Notes, 2003, vol. 324, p. 435 
Ramakrishnan V., Hovatta T., Nieppola E., Tornikoski M., Lähteenmäki A., Valtaoja E., Locating the $\gamma$-ray emission site in Fermi/LAT blazars from correlation analysis between $37 \mathrm{GHz}$ radio and $\gamma$-ray light curves, Monthly Notices of the Royal Astronomical Society, 2015, vol. 452, p. 1280

Rani B., Radio Galaxies-The TeV Challenge, Galaxies, 2019, vol. 7, p. 23

Rees M. J., Begelman M. C., Blandford R. D., Phinney E. S., Ion-supported tori and the origin of radio jets, Nature, 1982, vol. 295, p. 17

Ricci F., Massaro F., Landoni M., D’Abrusco R., Milisavljevic D., Stern D., Masetti N., Paggi A., Smith H. A., Tosti G., Optical Spectroscopic Observations of Gamma-ray Blazar Candidates. IV. Results of the 2014 Follow-up Campaign, The Astronomical Journal, 2015, vol. 149, p. 160

Richter G., Search for Optical Identifications in the 5C3-Radio Survey II. Statistical Treatment and Results, Astronomische Nachrichten, 1975, vol. 296, p. 65

Rieger F. M., NONTHERMAL PROCESSES IN BLACK HOLE-JET MAGNETOSPHERES-INVITED REVIEW, International Journal of Modern Physics D, 2011, vol. 20, p. 1547

Romani R. W., Watters K. P., Constraining pulsar magnetosphere geometry with $\gamma$-ray light curves, The Astrophysical Journal, 2010, vol. 714, p. 810

Rybicki G. B., Lightman A. P., Radiative processes in astrophysics. John Wiley \& Sons, 2008

Salvetti D., Chiaro G., La Mura G., Thompson D. J., 3FGLzoo: classifying 3FGL unassociated Fermi-LAT $\gamma$-ray sources by artificial neural networks, Monthly Notices of the Royal Astronomical Society, 2017, vol. 470, p. 1291

Sambruna R., Gliozzi M., Eracleous M., Brandt W., Mushotzky R., The XMM-Newton view of the nucleus of NGC 4261, ApJ, 2003, vol. 586, p. L37

Schinzel F. K., Petrov L., Taylor G. B., Mahony E. K., Edwards P. G., Kovalev Y. Y., New associations of gamma-ray sources from the Fermi second source catalog, The Astrophysical Journal Supplement Series, 2015, vol. 217, p. 4 
Schlafly E. F., Finkbeiner D. P., Measuring reddening with Sloan Digital Sky Survey stellar spectra and recalibrating SFD, The Astrophysical Journal, 2011, vol. 737, p. 103

Schmidt M., Optical Spectra and Redshifts of 31 Radio Galaxies., The Astrophysical Journal, 1965, vol. 141, p. 1

Shakura N. I., Sunyaev R. A., Black holes in binary systems. Observational appearance., Astronomy and Astrophysics, 1973, vol. 24, p. 337

Sikora M., Begelman M. C., Magnetic flux paradigm for radio loudness of active galactic nuclei, The Astrophysical Journal Letters, 2013, vol. 764, p. L24

Sironi L., Spitkovsky A., Relativistic Reconnection: An Efficient Source of Non-thermal Particles, ApJ, 2014, vol. 783, p. L21

Spada M., Ghisellini G., Lazzati D., Celotti A., Internal shocks in the jets of radio-loud quasars, Monthly Notices of the Royal Astronomical Society, 2001, vol. 325, p. 1559

Stecker F. W., Cosmic gamma rays. vol. 249, Scientific and Technical Information Office, National Aeronautics and Space Administration, 1971

Stickel M., Padovani P., Urry C., Fried J., Kühr H., The complete sample of 1 Jansky BL Lacertae objects. I-Summary properties, The Astrophysical Journal, 1991, vol. 374, p. 431

Stocke J. T., Morris S. L., Gioia I., Maccacaro T., Schild R., Wolter A., Fleming T. A., Henry J. P., The Einstein observatory extended medium-sensitivity survey. II-the optical identifications, The Astrophysical Journal Supplement Series, 1991, vol. 76, p. 813

Sturrock P., A model of pulsars, The Astrophysical Journal, 1971, vol. 164, p. 529

Sutherland W., Saunders W., On the likelihood ratio for source identification, Monthly Notices of the Royal Astronomical Society, 1992, vol. 259, p. 413

Swanenburg B., Bennett K., Bignami G., Caraveo P., Hermsen W., Kanbach G., Masnou J., Mayer-Hasselwander H., Paul J., Sacco B., et al., COS B observation of high-energy $\gamma$ radiation from 3C273, Nature, 1978, vol. 275, p. 298 
Takami H., $\gamma$-rays as a diagnostic of the origin of core radiation in low-luminosity active galactic nuclei, Monthly Notices of the Royal Astronomical Society, 2011, vol. 413, p. 1845

Tam P.-H. T., Hui C. Y., Kong A. K., Gamma-ray Emission from Globular Clusters, Journal of Astronomy and Space Sciences, 2016, vol. 33, p. 1

Tanada K., Kataoka J., Arimoto M., Akita M., Cheung C. C., Digel S. W., Fukazawa Y., The Origins of the Gamma-Ray Flux Variations of NGC 1275 Based on Eight Years of Fermi-LAT Observations, ApJ, 2018, vol. 860, p. 74

Tauris T. M., Savonije G. J., Formation of millisecond pulsars. I. Evolution of low-mass X-ray binaries with P_orb\&gt; 2 days, A\&A, 1999, vol. 350, p. 928

Tavani M., Ten years of AGILE: the mission and scientific highlights, Rendiconti Lincei. Scienze Fisiche e Naturali, 2019, vol. 30, p. 13

Tavani M., Barbiellini G., Argan A., Bulgarelli A., Caraveo P., Chen A., Cocco V., Costa E., De Paris G., Del Monte E., et al., The AGILE space mission, Nuclear Instruments and Methods in Physics Research Section A: Accelerators, Spectrometers, Detectors and Associated Equipment, 2008, vol. 588, p. 52

Tchekhovskoy A., Launching of Active Galactic Nuclei Jets. In Astrophysics and Space Science Library, vol. 414 of Astrophysics and Space Science Library, 2015, p. 45

Tegmark M., Strauss M. A., Blanton M. R., Abazajian K., Dodelson S., Sandvik H., Wang X., Weinberg D. H., Zehavi I., Bahcall N. A., et al., Cosmological parameters from SDSS and WMAP, Physical Review D, 2004, vol. 69, p. 103501

Teng S. H., Mushotzky R. F., Sambruna R. M., Davis D. S., Reynolds C. S., FERMI/LAT OBSERVATIONS OF SWIFT/BAT SEYFERT GALAXIES: ON THE CONTRIBUTION OF RADIO-QUIET ACTIVE GALACTIC NUCLEI TO THE EXTRAGALACTIC $\gamma$-RAY BACKGROUND, The Astrophysical Journal, 2011, vol. 742, p. 66

Thompson D., Bertsch D., Fichtel C., Hartman R., Hofstadter R., Hughes E., Hunter S., Hughlock B., Kanbach G., Kniffen D., et al., Calibration of the energetic gamma-ray 
experiment telescope (EGRET) for the Compton gamma-ray observatory, The astrophysical Journal supplement series, 1993, vol. 86, p. 629

Thompson D., Fichtel C., Extragalactic gamma radiation-Use of galaxy counts as a galactic tracer, Astronomy and Astrophysics, 1982, vol. 109, p. 352

Timmes F., Woosley S., Weaver T. A., Galactic chemical evolution: Hygrogen through zinc, Astrophysical Journal Supplement Series, 1995, vol. 98

Tody D., The IRAF data reduction and analysis system. In Instrumentation in astronomy VI , vol. 627,1986, p. 733

Tremaine S., Gebhardt K., Bender R., Bower G., Dressler A., Faber S. M., Filippenko A. V., Green R., Grillmair C., Ho L. C., Kormendy J., Lauer T. R., Magorrian J., Pinkney J., Richstone D., The Slope of the Black Hole Mass versus Velocity Dispersion Correlation, ApJ, 2002, vol. 574, p. 740

Tridon D. B., Schweizer T., Goebel F., Mirzoyan R., Teshima M., Collaboration M., et al., The MAGIC-II gamma-ray stereoscopic telescope system, Nuclear Instruments and Methods in Physics Research Section A: Accelerators, Spectrometers, Detectors and Associated Equipment, 2010, vol. 623, p. 437

Urpin V., Geppert U., Accretion and evolution of the neutron star magnetic field, Monthly Notices of the Royal Astronomical Society, 1995, vol. 275, p. 1117

Urry C. M., Padovani P., Unified schemes for radio-loud active galactic nuclei, Publications of the Astronomical Society of the Pacific, 1995, vol. 107, p. 803

Van Dokkum P. G., Cosmic-ray rejection by Laplacian edge detection, Publications of the Astronomical Society of the Pacific, 2001, vol. 113, p. 1420

van Oers P., Markoff S., Uttley P., McHardy I., van der Laan T., Donovan Meyer J., Connors R., A jet-dominated model for a broad-band spectral energy distribution of the nearby low-luminosity active galactic nucleus in M94, MNRAS, 2017, vol. 468, p. 435

Veledina A., Poutanen J., Vurm I., Hot accretion flow in black hole binaries: a link connecting X-rays to the infrared, Monthly Notices of the Royal Astronomical Society, 2013, vol. 430 , p. 3196 
Venturi T., Giovannini G., Feretti L., Comoretto G., Wehrle A., VLBI observations of a complete sample of radio galaxies. II-The parsec-scale structure of NGC 315, ApJ, 1993, vol. 408 , p. 81

Verbunt F., Binary Evolution and Neutron Stars in Globular Clusters. In New Horizons in Globular Cluster Astronomy, vol. 296, 2003a, p. 245

Verbunt F., New Horizons in Globular Cluster Astronomy (ASP Conf. Ser. 296), ed, G. Piotto, G. Meylan, SG Djorgovski, \& M. Riello (San Francisco, CA: ASP), 2003b, vol. 245

Verbunt F., Freire P. C., On the disruption of pulsar and X-ray binaries in globular clusters, Astronomy \& Astrophysics, 2014, vol. 561, p. A11

Verbunt F., Hut P., The globular cluster population of x-ray binaries. In SymposiumInternational Astronomical Union, vol. 125, 1987, p. 187

Verdoes Kleijn G. A., Baum S. A., de Zeeuw P. T., O'Dea C. P., Core radio and optical emission in the nuclei of nearby FR I radio galaxies, AJ, 2002, vol. 123, p. 1334

Walker R., Dhawan V., Romney J., Kellermann K., Vermeulen R. C., VLBA absorption imaging of ionized gas associated with the accretion disk in NGC 1275, ApJ, 2000, vol. 530 , p. 233

Weekes T. C., Cawley M. F., Fegan D., Gibbs K., Hillas A., Kowk P., Lamb R., Lewis D., Macomb D., Porter N., et al., Observation of tev gamma-rays from the crab nebula using the atmospheric cherenkov imaging technique, Astrophysical Journal, 1989, vol. 342, p. 379

White R. L., Becker R. H., Helfand D. J., Gregg M. D., A catalog of 1.4 GHz radio sources from the FIRST survey, The Astrophysical Journal, 1997, vol. 475, p. 479

Wojaczyński R., Niedźwiecki A., Xie F.-G., Szanecki M., Gamma-ray activity of Seyfert galaxies and constraints on hot accretion flows, A\&A, 2015, vol. 584, p. A20

Wolstencroft R., Savage A., Clowes R., MacGillivray H., Leggett S., Kalafi M., The identification of IRAS point sources-I. A 304 deg2 field centred on the South Galactic Pole, Monthly Notices of the Royal Astronomical Society, 1986, vol. 223, p. 279 
Woo J.-H., Urry C. M., Active galactic nucleus black hole masses and bolometric luminosities, ApJ, 2002, vol. 579, p. 530

Wood M., Caputo R., Charles E., Di Mauro M., Magill J., et al., Fermipy: An open-source Python package for analysis of Fermi-LAT Data, arXiv preprint arXiv:1707.09551, 2017

Worrall D., Birkinshaw M., O'sullivan E., Zezas A., Wolter A., Trinchieri G., Fabbiano G., The jet and counterjet of 3C 270 (NGC 4261) viewed in the X-ray with Chandra, MNRAS, 2010, vol. 408, p. 701

Wright E. L., Eisenhardt P. R., Mainzer A. K., Ressler M. E., Cutri R. M., Jarrett T., Kirkpatrick J. D., Padgett D., McMillan R. S., Skrutskie M., et al., The Wide-field Infrared Survey Explorer (WISE): mission description and initial on-orbit performance, The Astronomical Journal, 2010, vol. 140, p. 1868

Wrobel J., Heeschen D., Radio-continuum sources in nearby and bright E/S0 galaxiesActive nuclei versus star formation, AJ, 1991, vol. 101, p. 148

Yang R.-z., Kafexhiu E., Aharonian F., Exploring the shape of the $\gamma$-ray spectrum around the $\pi^{0}$-bump, Astronomy \& Astrophysics, 2018, vol. 615, p. A108

York D. G., Adelman J., Anderson Jr J. E., Anderson S. F., Annis J., Bahcall N. A., Bakken J., Barkhouser R., Bastian S., Berman E., et al., The sloan digital sky survey: Technical summary, The Astronomical Journal, 2000, vol. 120, p. 1579

Young A. J., Wilson A., Mundell C., Chandra imaging of the x-ray core of the Virgo cluster, ApJ, 2002, vol. 579, p. 560

Yu Z., Yuan F., Ho L. C., On the Origin of Ultraviolet Emission and the Accretion Model of Low-luminosity Active Galactic Nuclei, ApJ, 2011, vol. 726, p. 87

Yuan F., Cui W., Radio-X-Ray Correlation and the "Quiescent State" of Black Hole Sources, ApJ, 2005, vol. 629, p. 408

Yuan F., Narayan R., Hot accretion flows around black holes, Annual Review of Astronomy and Astrophysics, 2014, vol. 52, p. 529 
Zabalza V., naima: a Python package for inference of relativistic particle energy distributions from observed nonthermal spectra, Proc. of International Cosmic Ray Conference 2015, 2015, p. 922

Zezas A., Birkinshaw M., Worrall D., Peters A., Fabbiano G., Chandra observations of NGC 4261 (3C 270): revealing the jet and hidden active galactic nucleus in a Type 2 LINER, ApJ, 2005, vol. 627, p. 711 